\title{
Myelin Membrane Growth and Organization in a Cellular Model System
}

\author{
PhD Thesis \\ in partial fulfillment of the requirements \\ for the degree "Doctor of Philosophy (PhD)" \\ in the Molecular Biology Program \\ at the Georg August University Göttingen, \\ Faculty of Biology
}

submitted by

Larisa Yurlova

born in

St. Petersburg, Russian Federation

Göttingen 2010 
Thesis Committee:

Prof. Mikael Simons

Prof. Detlef Doenecke

Prof. Reinhard Jahn 


\title{
Affidavit
}

I hereby declare that this $\mathrm{PhD}$ thesis 'Myelin Membrane Growth and Organization in a Cellular Model System' has been written independently with no other aids or sources than quoted. This thesis (wholly or in part) has not been submitted elsewhere for any academic award or qualification.

\author{
Larisa Yurlova
}

June, 2010

Göttingen, Germany 


\section{Related Publications}

The results of this project will be communicated in the following publications. I am grateful to all co-authors for the stimulating and fruitful collaborations.

- Yurlova L, Aggarwal S, Zimmermann J, Chiantia S, Kahya N, Kaiser H, Bruegger B, Simons M. Restricting protein diffusion permits self-organization of lipids for myelin membrane assembly. In preparation

- Yurlova L, Steshenko L, Aggarwal S, Friedrichs J, Simons M. Trafficking and expansion of myelin membrane in primary oligodendrocytes. In preparation

- Nawaz S, Yurlova L, Manrique N, Schmidt S, Schaap I, Simons M. Myelin sheath spreads by relaxation of cortical tension through developmentally-regulated actin depolymerization. In preparation

Parts of the work described in this thesis have been presented at international meetings and symposia. I want to sincerely thank all people who contributed to these studies.

- Yurlova L, Zimmermann J, Chiantia S, Aggarwal S, Bruegger B, Simons M. Myelin membrane trafficking in a cellular model system. International Meeting on Intracellular Trafficking Processes, Weizmann Institute of Science, Rehovot, Israel, 3 - 5 May 2010, oral presentation

- Yurlova L, Aggarwal S, Zimmermann J, Simons M. Mechanisms of myelin membrane growth in a cellular model system. $49^{\text {th }}$ Annual Meeting of the American Society for Cell Biology, San Diego CA, USA, 5 - 9 December 2009, Poster No. 1528 
- Yurlova L, Aggarwal S, Zimmermann J, Simons M. Myelin membrane trafficking and expansion in oligodendrocytes. $6^{\text {th }}$ International $\mathrm{PhD}$ Student Symposium "Horizons in Molecular Biology 2009", Göttingen, Germany, 9 12 September 2009, Poster No. 60

- Yurlova L, Winter C, Simons M. MBP in regulation of endosomal trafficking of myelin membrane components. SFB523 Symposium on Intracellular Trafficking and Transport 2008. Göttingen, Germany, 3 - 4 April 2008, Poster No. 63 


\section{Contents}

Contents vii

List of Figures . . . . . . . . . . . . . . . . . . . xiii

List of Tables . . . . . . . . . . . . . . . . . . . xvii

Acknowledgements $\quad$ xix

$\begin{array}{ll}\text { Abstract } & \mathrm{xxi}\end{array}$

1 Introduction 1

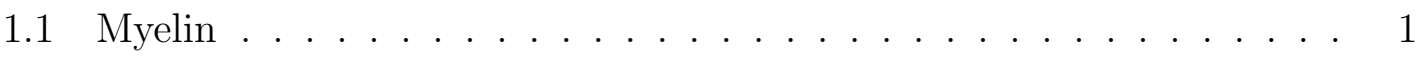

1.1.1 Morphological Structure of Myelin . . . . . . . . . . . 2

Evolution of the Myelin Sheath . . . . . . . . . . . 2

Conventional Myelin . . . . . . . . . . . . . . . 2

Myelin Structure in Development . . . . . . . . . . . . 3

Unconventional Myelin . . . . . . . . . . . . . . 4

1.1.2 Myelin-Producing Cells in the CNS . . . . . . . . . . . 5

Historical Overview . . . . . . . . . . . . . . . . 5

Origin of Oligodendrocytes . . . . . . . . . . . . . . 6

Oligodendroglial Lineage . . . . . . . . . . . . . . . 7

1.2 Molecular Composition of Myelin . . . . . . . . . . . . . . . . 8

1.2.1 Lipid Composition of Myelin . . . . . . . . . . . . . 8

Sphingolipids: Galactosylceramide and Sulfogalactosylceramide 10

Cholesterol . . . . . . . . . . . . . . . . . . 12

1.2.2 Myelin Proteins . . . . . . . . . . . . . . . . . . . . 13

Myelin Basic Protein (MBP) . . . . . . . . . . . . . . 13

Proteolipid Protein $(\mathrm{PLP}) \ldots \ldots \ldots$. . . . . . . . . 14 
Myelin-Associated Glycoprotein (MAG) . . . . . . . . 15

Myelin Oligodendrocyte Glycoprotein (MOG) . . . . . . . 16

2',3'-Cyclic-Nucleotide 3'-Phosphodiesterase (CNPase) . . . . 16

Other Proteins of the CNS Myelin . . . . . . . . . . . . 17

1.3 Intracellular Trafficking to the Plasma Membrane . . . . . . . . . . . 18

1.3.1 Intracellular Trafficking Pathways . . . . . . . . . . . . . . . . 19

Biosynthetic-Secretory Pathway . . . . . . . . . . . . . . 19

Exocytosis and its Vesicular Fusion Machinery . . . . . . . . 21

Endocytic Pathway . . . . . . . . . . . . . . . 24

1.3.2 Trafficking in Polarized Cells, Specifics of Trafficking in Oligo-

dendrocytes . . . . . . . . . . . . . . . 26

Common Mechanisms for Establishing Cell Polarity . . . . . . 26

Biosynthetic Pathways of the Main Myelin Components . . . . 27

Exocytic Pathway in Myelin Production . . . . . . . . . . 29

Endocytic Pathway in Myelin Membrane Formation . . . . . . 30

Oligodendroglial Cytoskeleton . . . . . . . . . . . . . 31

1.4 Membrane Organization . . . . . . . . . . . . . . . 32

1.4.1 Cell Membrane Models . . . . . . . . . . . . . . . . . 32

1.4.2 Phase Separation Concept . . . . . . . . . . . . . 33

1.4.3 Common Mechanisms of Membrane Domain Assembly . . . . 35

1.4.4 Specifics of Myelin Membrane Domain Organization . . . . . . 36

1.5 Aims and Objectives . . . . . . . . . . . . . . . 38

2 Materials \& Methods $\quad 39$

2.1 Materials . . . . . . . . . . . . . . . . . . . . 39

2.1.1 Chemicals and Consumables . . . . . . . . . . . . . 39

2.1.2 Antibodies . . . . . . . . . . . . . . . . 39

2.1.3 DNA Constructs . . . . . . . . . . . . . . . . . . 41

2.1.4 General Buffers and Solutions . . . . . . . . . . . . . . . . 42

Phosphate Buffered Saline (PBS) . . . . . . . . . . . 42

Protease Inhibitors $(\mathrm{PI}) \quad \ldots \ldots \ldots$

Inhibitors for Cell Culture Assays . . . . . . . . . . . . . . . 42 
2.1.5 Commercial Kits . . . . . . . . . . . . . . . . . . . . . 42

2.1.6 Specific Software . . . . . . . . . . . . . . 43

2.2 Methods . . . . . . . . . . . . . . . . . . . . . . . . 43

2.2.1 Molecular Biology . . . . . . . . . . . . . . . . . 43

Extraction and Purification of Plasmid DNA . . . . . . . . 44

Determination of DNA and RNA Concentration . . . . . . 45

DNA Analysis with Agarose Gels . . . . . . . . . . . . 46

DNA Extraction from Agarose Gels . . . . . . . . . . . . 46

Restriction Enzyme Digestion . . . . . . . . . . . . . 47

DNA Ligation . . . . . . . . . . . . . . . . . 47

Polymerase Chain Reaction (PCR) . . . . . . . . . . 48

Primer Design . . . . . . . . . . . . . . . . 5 50

Site-Directed Mutagenesis . . . . . . . . . . . . . 51

Transformation of Competent E. coli . . . . . . . . . . . 51

DNA Sequencing . . . . . . . . . . . . . . 51

Cloning of Viral Vectors, Production of Recombinant SFV . . 52

Total RNA Isolation . . . . . . . . . . . . . . . . 53

Denaturing Agarose Gel Electrophoresis of RNA . . . . . . . . 53

cDNA Synthesis . . . . . . . . . . . . . . . 54

Quantitative Real-Time PCR . . . . . . . . . . 55

Shiverer Mouse Line Maintenance and Genotyping . . . . . . 57

2.2.2 Biochemistry . . . . . . . . . . . . . . . . . 59

Sodium Dodecyl Sulfate Polyacrylamide Gel Electrophoresis

(SDS-PAGE) . . . . . . . . . . . . . . 59

Western Blotting . . . . . . . . . . . . . 61

Preparation of Cells for Biochemical Analysis . . . . . . . . 62

Preparation of Total Membrane Fractions . . . . . . . . 63

Preparation of Myelin Fractions . . . . . . . . . . . . 63

Lipid Extraction . . . . . . . . . . . . . . 65

Determination of Protein Concentration . . . . . . . . 65

Detergent Solubility Assays _ . . . . . . . . . . . . . 66

Lipid Analysis . . . . . . . . . . . . . . . . . . . . 67 
Fluorescence Correlation Spectroscopy (FCS) . . . . . . . 68

Generation of Giant Unilamellar Vesicles (GUVs) . . . . . . . 68

Membrane Order Analysis with C-Laurdan . . . . . . . . 68

2.2.3 Cell Culture Techniques . . . . . . . . . . . . . . 69

Primary Oligodendrocyte Culture . . . . . . . . . . . . 69

Growth and Maintenance of Oli-neu Cell Line . . . . . . . . 70

Growth and Maintenance of Baby Hamster Kidney Cell Line

(BHK21) . . . . . . . . . . . . . 71

MAG513 Antibody Production with Hybridoma Cell Line . . 71

Cryopreservation, Storage and Thawing of Cell Lines . . . . . 72

Methods for Nucleic Acids Delivery into Cultured Animal Cells 72

Immunocytochemistry . . . . . . . . . . . . . . . 74

2.2.4 Microscopy, Image Processing and Statistical Analysis . . . . . 75

Confocal Microscopy . . . . . . . . . . . . . 75

Image Analysis . . . . . . . . . . . . . . . . 76

Atomic Force Microscopy $(\mathrm{AFM})$. . . . . . . . . . 77

Fluorescence Recovery After Photobleaching (FRAP) . . . . . 77

Total Internal Reflection Fluorescence (TIRF) Microscopy . . 80

Statistical Analysis . . . . . . . . . . . . . . . 80

3 Results $\quad 83$

3.1 Characterization of the Cellular Model System . . . . . . . . . . . 83

3.1.1 Immunocytochemical Localization of Myelin-Specific Proteins and Lipids in pOLs . . . . . . . . . . . . . . . . . 83

3.1.2 Biochemical Separation of Myelin and pOLs Membranes into Fractions with Distinct Protein Composition . . . . . . . . 87

3.1.3 Ultrastructure of Myelin Sheets in pOLs . . . . . . . . . . . 89

3.2 Plasma Membrane Extension in Primary Oligodendrocytes . . . . . . 90

3.2.1 How are Myelin Sheets Produced During Development? . . . . 90

Stages of Myelin Sheet Expansion: "Spider Web", "Half-Moons",

"Sheets" ..................... . . 92 
PLP Incorporation into the Plasma Membrane of Developing pOLs ............................... 94

Changes in Actin Cytoskeleton . . . . . . . . . . . . 95

3.2.2 Distribution of Membrane Extension Players in pOLs . . . . . 96

Localization of Biosynthetic Machinery . . . . . . . . . . 97

Localization of Vesicular Trafficking Machinery . . . . . . . . 97

Localization of Vesicular Fusion Regulators . . . . . . . . . . . 100

3.2.3 Where does Vesicular Trafficking Take Place in pOLs? . . . . 103

3.2.4 Thickness of Myelin Membrane Sheets . . . . . . . . . . . . 106

3.2.5 Mapping Fusion Sites on pOLs Plasma Membrane . . . . . . . 106

3.2.6 Lateral Diffusion on Plasma Membrane of pOLs . . . . . . . . 108

3.3 Molecular Mechanisms of Myelin Membrane Organization . . . . . . . 110

3.3.1 Properties of Membrane Domains in pOLs . . . . . . . . . . 111

Morphological and Biochemical Visualization of Membrane

Domains in pOLs . . . . . . . . . . . . . 111

Lipids in Myelin Domains of pOLs . . . . . . . . . . 113

3.3.2 Depletion Studies on Myelin Membrane Organization in pOLs and in CNS Myelin . . . . . . . . . . . . . 119

Sphingolipid Depletion Results in Intermixing of Membrane Domains in pOLs . . . . . . . . . . . . . . . 119

CerS2 Deletion Changes Physical Properties of Myelin Membrane ...................... 120

Sphingolipid Depletion Decreases MBP Production by pOLs . 122

MBP Deletion Interferes with pOLs Membrane Domain Organization . . . . . . . . . . . . . . . 123

4 Discussion

4.1 Cell Culture Model to Study Myelin Sheath: Model Validation . . . . 133

4.1.1 Morphological Approach . . . . . . . . . . . . . . . . 133

4.1.2 Biochemical Approach to Study Myelin Membrane . . . . . . 135

4.2 Mechanisms of Myelin Sheet Growth in pOLs . . . . . . . . . . . . . 136

4.2.1 Maturation Stages in Culture . . . . . . . . . . . . . 136 
4.2.2 Actin Cytoskeleton in Myelin Sheet Expansion . . . . . . . . . 139

4.3 New Membrane Incorporation into a Growing Myelin Sheet . . . . . . 142

4.3.1 Indications from Development . . . . . . . . . . . . . . . . . . 142

4.3.2 Subcellular Localization of Membrane Extension Players . . . 143

4.3.3 Hypothesis: Proximal Incorporation, Distal Flow . . . . . . . 144

4.4 Mechanisms of Myelin Domain Assembly . . . . . . . . . . . . . . . 146

4.4.1 Role of Myelin Sphingolipids in Domain Formation . . . . . . 148

4.4.2 Regulation of MBP Levels by Sphingolipids . . . . . . . . . . 149

4.4.3 MBP Depletion in Cell Culture and In Vivo . . . . . . . . . . 150

4.4.4 Lateral Segregation of Myelin Lipids in Model Membranes . . 151

4.4.5 Phase Separation in the Context of Myelin Biogenesis . . . . . 152

5 Summary and Conclusions

A Sequences

A.1 Sequences of Tags and Peptides . . . . . . . . . . . . . 157

A.2 Sequences of Cloned Plasmids . . . . . . . . . . . . . . . . . 157

$\begin{array}{ll}\text { B Source Code } & 161\end{array}$

B.1 ImageJ Macros . . . . . . . . . . . . . . . . . . . . . . . 161

B.1.1 Calculation of the Correlation Coefficient Between the Two Channels . . . . . . . . . . . . . . . . . . 161

B.1.2 Measuring Total Fluorescence Intensities . . . . . . . . . . . . 162

B.1.3 Measuring ROI Fluorescence Intensities . . . . . . . . . . . . . 162

B.1.4 Image Processing, Cropping, Channel Merge . . . . . . . . . . 163

C Supplementary Figures

D List of Symbols and Abbreviations $\quad 173$

$\begin{array}{lr}\text { Bibliography } & 177\end{array}$

$\begin{array}{ll}\text { Curriculum Vitae } & 209\end{array}$ 


\section{List of Figures}

3.1 Immunocytochemical localization of the non-compact myelin proteins MAG and CNPase in pOLs . . . . . . . . . . . . . . . . 84

3.2 Immunocytochemical localization of the compact myelin proteins PLP and MOG in pOLs . . . . . . . . . . . . . . . 85

3.3 Immunocytochemical localization of galactosphingolipids in pOLs . 86

3.4 Quantification of colocalization of compact and non-compact myelin proteins in pOLs . . . . . . . . . . . . . . . . . 87

3.5 Western blot analysis of subfractions of different densities, isolated from myelin and from pOLs membranes . . . . . . . . . . . . . . 88

3.6 Immunoelectron microscopy of cultured primary oligodendrocytes . 89

3.7 Stages of myelin sheet development: morphological changes in culture 90

3.8 Stages of myelin sheet development: PLP incorporation into plasma membrane ......................... . . 91

3.9 Cultured primary oligodendrocytes at a "half-moon" stage . . . . . 93

3.10 Total and surface PLP pools in developing oligodendrocytes . . . . 94

3.11 Microfilaments in developing and mature oligodendrocytes . . . . . 96

3.12 Subcellular distribution of the biosynthetic machinery in primary oligodendrocytes . . . . . . . . . . . . . . . . . . . . . . . . . 98

3.13 Subcellular distribution of microtubules and endosomes in pOLs . . 99

3.14 Localization of SNARE proteins syntaxins 2 - 4 in pOLs . . . . . 100

3.15 Localization of SNARE proteins SNAP-23 and VAMP2 in pOLs . . 101

3.16 Localization of Rab3 and Rab3a in pOLs . . . . . . . . . . . . . . . 102

3.17 Localization of exocyst and signaling lipid PIP2 in pOLs . . . . . . 103

3.18 Localization of clathrin and caveolin in pOLs . . . . . . . . . . . . . 104 
3.19 Discrimination of intracellular and surface pools of myelin protein

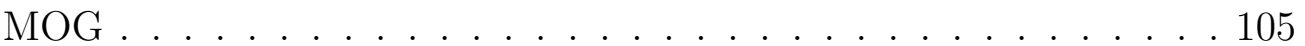

3.20 Thickness of myelin-like membrane sheets measured with AFM in pOLs . . . . . . . . . . . . . . . . . 107

3.21 Brefeldin A treatment slows down lateral diffusion of GalC on pOLs plasma membrane . . . . . . . . . . . . . . . . . . . . . 108

3.22 FRAP rates in myelin-like membrane sheets proximally and distally to the processes . . . . . . . . . . . . . . . . . . . . 109

3.23 Visualization of distinct plasma membrane domains in pOLs with confocal microscopy . . . . . . . . . . . . . . . . . . . . . . . . . . . 112

3.24 CHAPS solubility of myelin proteins MBP, PLP, MOG and MAG . 114

3.25 Analysis of lipid classes in membrane subfractions from pOLs cultures115

3.26 Lipid order analysis in membrane subfractions from isolated myelin and from cultured pOLs . . . . . . . . . . . . . . 116

3.27 Lipid order analysis in purified myelin and in Oli-neu membranes . 117

3.28 Self-organization of myelin lipids results in lateral segregation in model membranes . . . . . . . . . . . . . . . . . . . . . . . . . . . . 118

3.29 Redistribution of PLP signal in membrane subfractions from pOLs treated with FB1: densitometric analysis of Western blots . . . . . . 120

3.30 Membrane order and diffusion properties of CerS2 KO myelin . . . 121

3.31 Depletion of sphingolipids results in MAG redistribution in pOLs . . 122

3.32 Sphingolipid depletion causes reduction of MBP amounts in pOLs . 124

3.33 Relative MBP expression levels in pOLs after FB1 treatment . . . . 125

3.34 MBP protein stability is reduced in sphingolipid depletion conditions126

3.35 MBP deficiency results in loss of membrane asymmetry in primary oligodendrocytes: MAG and CNPase redistribution in Shiverer pOLs127

3.36 Quantitative analysis of MAG and CNPase distribution in Shiverer pOLs . . . . . . . . . . . . . . . . . . . . 128

3.37 Redistribution of PLP signal in Shiverer crude myelin subfractions . 129

3.38 Membrane order and diffusion properties of MBP-deficient Shiverer crude myelin . . . . . . . . . . . . . . . . . . . . . . . . 130

3.39 Cytoskeleton in Shiverer pOLs . . . . . . . . . . . . . . . 131 
4.1 Schematic representation of myelin sheet development in culture . . 137

4.2 Schematic representation of the myelin growth hypothesis . . . . . . 145

C.1 Endocytosis in developing oligodendrocytes: CTX uptake . . . . . . 165

C.2 Endocytosis of MAG, MOG, GalC and PLP epitopes . . . . . . . 166

C.3 Quantitative analysis of endocytosis of MAG, MOG and GalC . . . 167

C.4 Subcellular localization of overexpressed VSVG-YFP, MOG-GFP and MBP-GFP in pOLs . . . . . . . . . . . . . . . . . 168

C.5 Subcellular localization of overexpressed GFP and RFP in pOLs . . 169

C.6 Quantitative analysis of lipid molecular species in membrane subfractions from cultured primary oligodendrocytes . . . . . . . . . . 170

C.7 FRAP analysis: free lipid diffusion in the myelin sheet . . . . . . . . 171 


\section{List of Tables}

1.1 Comparison of lipid composition of CNS myelin and brain . . . . 9

$2.1 \quad$ Antibodies .......................... . . 40

2.2 DNA constructs, acquired . . . . . . . . . . . . . . . 41

2.3 DNA constructs, cloned . . . . . . . . . . . . . . . 41

2.4 Inhibitors . . . . . . . . . . . . . . . . . . . . 43

2.5 Commercial kits . . . . . . . . . . . . . . . . 43

2.6 List of software . . . . . . . . . . . . . . . . . . 44

2.7 Primers for real-time PCR . . . . . . . . . . . . . 57

2.8 Primers for Shiverer genotyping . . . . . . . . . . . . . . . 58

A.1 Sequences of tags and peptides . . . . . . . . . . . . 157

A.2 Nucleotide sequence of myc-thrombin-HA-MOG (clone No 62) . . . 158

A.3 List of primers used for cloning . . . . . . . . . . . . . . . . . . . . 159

A.4 Nucleotide sequence of pHluorin-thrombin-MOG (clone No 82) . . . 160 


\section{Acknowledgements}

I am very grateful and indebted to my scientific advisor, Prof. Dr. Mikael Simons, for his supervision, open mind, for supporting my ideas and for providing an outstanding research environment in his laboratory at the Max Planck Institute of Experimental Medicine. I am grateful to the members of my thesis committee: Prof. Detlef Doenecke, Prof. Reinhard Jahn, Prof. Klaus-Armin Nave for fruitful discussions and scientific guidance.

I want to express my gratitude to the Coordination Office of $\mathrm{MSc} / \mathrm{PhD}$ Molecular Biology Program: Steffen Burkhardt and his assistants Nina McGuinness, Ivana Bacakova, Kerstin Grüniger for superior organization of the graduate program and for unconditional support of their students. I would also like to thank my supervisors of short-term research projects (labrotations) Dr. Etienne Herzog, Dr. Dorothea Brandhorst and Prof. Sigrid Hoyer-Fender for all the hands-on experience in molecular biology, microscopy and protein biochemistry they passed on to me. I am grateful to Prof. Ahmed Mansouri for giving me the opportunity to master the art of designing and cloning complex gene-targeting vectors at his laboratory. These skills and experience which I gained during the first two years of my studies within the MSc/PhD Molecular Biology Program helped me a lot in pursuing my doctoral project.

It has been a pleasure to work with all the members of AG Simons and I want to thank each of them for a great working atmosphere and ideas exchange: Angelika Kippert, Mareike Schnaars, Sebastian Schmidt, Natalia Manrique, Mostafa Bakhti, Katarina Trajkovic, Aniket Ghosh, Dirk Fitzner, Anja Schneider, Katrin Strauss and Holger Budde. Special thanks to Giselheid Schulz for brilliant oligodendrocytes, Shweta Aggarwal and Johannes Zimmermann for joining the myelin growth project when it started overgrowing, Chieh Hsu for reliability and help with wet-lab and in silico methods, and Lena Steshenko for being my motivated labrotation student.

I want to sincerely thank our collaborators: Salvatore Chiantia (London's lab, Stony Brook University, USA) for FCS measurements, Britta Brügger (Biochemistry Center of 
Heidelberg University, Heidelberg, Germany) for lipidomics, Nicoletta Kahya (University Medical Center, Groningen, Netherlands) for GUVs, Hermann-Josef Kaiser (Max Planck Institute of Molecular Cell Biology and Genetics, Dresden, Germany) for Laurdan measurements, Jonne Helenius and Jens Friedrichs (Biotechnology Center, TU-Dresden, Germany) for AFM measurements. I would also like to acknowledge my colleagues at the Max Planck Institutes in Göttingen for help with methodology, equipment, reagents, etc. My special gratitude goes to the NEUREST Coordinators for supporting my doctoral studies with the EU Marie Curie Fellowship. I am grateful to Open Source software developers for creating and ensuring free use of numerous applications for data processing, analysis and presentation.

I want to say thank you to many friends from $\mathrm{MSc} / \mathrm{PhD}$ Molecular Biology and Neuroscience Programs for networking and fun throughout the years of my doctoral study. To Alexandra Andreou, Gabriel Mora, Ioanna Bethani, Ramya Nair and Chieh Hsu for making Göttingen feel like my second home. To Florian Hauer, Burkhardt Heisen, Kathy Gelato, Marc Schneider, Christian Stegmann and Martin Schmeisser for many wonderful evenings, full of scientific discussions and cultural experiences. To Ben Frank, a person who means a lot to me, for sharing my joy and supporting in the hard times, for adding passion and adventure to my Göttingen days and for so much more. To my family, my all time friends, my advisors and teachers in Russia. 


\section{Abstract}

This thesis addresses the two fundamental questions of myelin biogenesis: how the myelin membrane is extended and how it acquires its specific lateral organization. Myelin is a multilayered membrane sheath, which oligodendrocytes wrap around axons to ensure rapid nerve conduction. To investigate molecular mechanisms of myelin membrane growth, we used primary cultures of myelin-producing cells, oligodendrocytes. We have characterized this cellular model system and found that it recapitulates the specific biochemical and morphological characteristics myelin acquires in vivo. Analogous to the situation in vivo, cultured primary oligodendrocytes develop distinct subcellular domains, namely plasma membrane sheets (resembling compact myelin) and processes (resembling non-compact myelin). Interestingly, oligodendrocytes establish these domains in cell culture even without wrapping around axons.

To gain insights into myelin membrane expansion mechanisms, we inspected vesicular transport and fusion machineries of oligodendrocytes. We found that biosynthetic organelles, cytoskeleton, exocyst and SNAREs are enriched in the processes and cell bodies of these cells and are almost excluded from the sheets. Further we determined that the vesicular transport pool of compact myelin proteins is also restricted to the processes and cell bodies of oligodendrocytes, whereas these proteins are distributed over the sheets when incorporated into the plasma membrane. Taken together, acquired data indicate that the newly produced myelin membrane is first integrated into the plasma membrane of the processes, but not in the sheets. This raises the question how plasma membrane asymmetry is achieved in oligodendrocytes. We analyzed lateral organization of oligodendroglial membranes and found that, both in culture and in vivo, segregation of the membrane proteins is guided by myelin basic protein (MBP), whose cellular levels are modulated by sphingolipids. 
In addition, we showed that myelin lipids can phase-separate from the total membrane lipids, implying the possibility for lipid self-organization in myelin. These data suggest a novel mechanism for myelin membrane formation: expansion from the processes into the sheet domains, followed by lateral organization under the control of membrane components (sphingolipids and MBP). 


\section{Chapter 1}

\section{Introduction}

\subsection{Myelin}

In our nervous system, nerves communicate through electric current. Analogous to electric wires, they are surrounded with insulation; this insulation is called myelin [1]. Myelin is formed by membrane outgrowths of specialized glial cells, wrapped around the nerve axons in a spiral fashion. Multilamellar myelin sheaths enable rapid saltatory (Latin saltare, "to jump") propagation of action potentials along ax-

ons. It speeds up nerve conduction, decreases metabolic costs of neural activity and dramatically reduces space requirements of the nervous system [2]. These benefits of myelin gave vertebrates a selective evolutionary advantage [3]. This advantage has, however, a price - a high level of complexity results in increased vulnerability, as demonstrated by diseases associated with myelin. Disturbance of myelin structure and function can be caused by acquired inflammatory and infectious diseases of myelin (e.g. multiple sclerosis), hereditary metabolic diseases (e.g. globoid cell leucodystrophy or Krabbe's disease), physical agent caused diseases (e.g. traumatic demyelination), etc. [4]. Myelin loss not only reduces nerve conduction velocity, but often leads to axonal degeneration, and can cause severe disabilities [5]. For understanding the pathophysiology of these diseases and designing treatments, we need detailed knowledge of the molecular mechanisms of myelin assembly and maintenance under normal conditions. 


\subsubsection{Morphological Structure of Myelin}

\section{Evolution of the Myelin Sheath}

Emergence of glial cells accompanied the evolution towards an increasingly centralized and cephalized systems of neurons. Neuroglia can be found in higher invertebrates, and some even possess distinct types of glial cells (e.g. insects) [6]. The most primitive invertebrate axons lack glial ensheathments, other axons are surrounded by a single row of elongated sheath cells, and in other cases axons have multilayered or spiral ensheathments [4]. In general, invertebrate glia displays a great variety of ensheathment patterns, with lamellae number ranging from zero (e.g. coelenterates) to several hundred (as in earthworm Lumbricus terrestris). Adjacent glial lamellae are often associated with one another by desmosome-like junctions [7]. Some large axons of invertebrates (e.g. prawn Palaemonetes vulgaris) even exhibit nodes, where each glial lamellae forms a specialized junction with the paranodal axolemma [8]. It seems that axon ensheathments have evolved independently in markedly different phyla. Although this relation between axon and glia can be found at specialized sites in some invertebrates, it is a regular feature in vertebrates. Vertebrate axon ensheathments have achieved a high level of structural and functional sophistication, forming structures called myelin [3]. In vertebrates, myelin can be even subdivided into CNS and PNS types. The PNS myelin sheath, composed of a compact glial lamellae that is wrapped around an axon was first observed in the chick sciatic nerve in the middle of the last century [9]. CNS myelin was reported a few years later as structured in a way principally similar to that of PNS [10].

\section{Conventional Myelin}

The mammalian CNS is composed of grey and white matter, the former contains neural cell bodies, and the latter consists mostly of myelinated tracts. While most axons in mammalian PNS are unmyelinated, the majority of CNS axons are myelinated [4]. Unmyelinated CNS axons have diameters up to $0.8 \mu \mathrm{m}$, while myelinated CNS axons have diameters starting with $0.2 \mu \mathrm{m}$ [11]. Myelin is a membrane structure that is spirally wrapped around axons and is constituted of the plasma membrane extensions of specialized myelinating glial cells, the oligodendrocytes in the CNS 
and the Schwann cells in the PNS [2]. In a cross-section, myelin shows a periodicity with alternating concentric electron-dense and light layers. The major dense line (dark layer) is formed by the apposition of the cytoplasmic surfaces of the myelin membrane [2]. The apposing outer surfaces of the myelin membrane form the minor dense lines (or intraperiodic lines). The periodicity of the lamellae is on average $12 \mathrm{~nm}$ [12], although depending on the species examined, axon diameter, and fixation technique, the myelin period has been reported to fall in a range between $8 \mathrm{~nm}$ to $16 \mathrm{~nm}$. Myelin sheath dimensions are coupled to axon diameter. Depending on the axon size, the mature CNS myelin sheath can have up to 160 compacted lamellae $[4]$.

The length of adult myelin segments averages to 150 - $200 \mu \mathrm{m}$ [12], although it can vary from $100 \mu \mathrm{m}$ to $1700 \mu \mathrm{m}$ [4]. Along a CNS myelinated fiber, one can distinguish nodes of Ranvier, internodes, and paranodal regions [13]. At the node of Ranvier, axolemma is not covered with myelin, but is exposed to the extracellular milieu. The length of the nodes of Ranvier is less than a micrometer [14], and at the nodal axolemma voltage-dependent $\mathrm{Na}^{+}$channels are clustered. Near the node of Ranvier, the myelin terminal loops containing cytoplasm are called paranodal loops. Each paranodal loop makes a contact with the axon, and the sequence of loops is named paranode. Paranodal junctions help to seal the internodal axon (and the periaxonal space between the innermost myelin layer and the axolemma) [15]. Internode myelin is as a rule compacted, with the Schmidt-Lanterman cytoplasmic incisures spanning the entire thickness of the large myelin sheath (these structures are common in the PNS, but rare in the CNS [4]). The innermost and outermost lamellae of the myelin sheath (the ends of the spiral) also retain cytoplasm, forming less compact inner (periaxonal) and outer (abaxonal) loops. In contrast to the PNS, the CNS myelin lacks an external basal lamina and exhibits a "radial component" - strands of junctions at the extracellular apposition, which localize in the sector between the inner and outer loops [4].

\section{Myelin Structure in Development}

The critical period of myelination varies significantly among different species. In rodents, myelination in the CNS occurs postnatally, predominantly in the first 4 weeks 
of life [16]. In humans, myelination begins during the second trimester and extends through the second year of life [17]. In developing CNS white matter oligodendrocytes extend processes, that contact, ensheath, and myelinate axons. In extensive EM reconstruction studies S. Remahl and C. Hildebrand have described the myelin sheaths extension and compaction processes [18, 19]. During initial myelination, axons become ensheathed by one or two uncompacted glial lamellae (E-sheath). Esheaths present a length range from $5 \mu \mathrm{m}$ to $150 \mu \mathrm{m}$ (studies done on the spinal cord and the corpus callosum of the cat [18]). Between successive E-sheaths naked axon portions occur, although these gaps are insufficient to allow elongation of all short sheaths into long sheaths, which implies the need for remodelling and elimination of the sheaths during development. After formation of maximally three layers of glial lamellae, compaction of the layers (transition from E-sheath to M-sheath) starts. Sheaths composed of mixed compacted (M-sheaths) and uncompacted segments have a length range of $66 \mu \mathrm{m}$ to $212 \mu \mathrm{m}$ [18]. The transition to partially compacted sheaths (transition from E to E-M-E) often takes place at the length of about $50 \mu \mathrm{m}$ in the callosal area and $100 \mu \mathrm{m}$ in the spinal cord, and E-M-E sheaths subsequently continue to elongate. In the callosal area, the transition from partially compacted (E-M-E) to fully compacted (M) sheaths happens at a length of $150 \mu \mathrm{m}$ and at lamellae number around 10, and this process includes gradual spreading of compaction towards the ends of the sheaths, formation of axoglial junctions and, finally, emergence of nodes of Ranvier [18]. Therefore, during myelination the length of the internodes, the diameter of the axons and the number of the myelin layers increase, suggesting that myelin expands in all planes at once [2]. Furthermore, compaction of the myelin sheath starts after three layers and is completed already after ten myelin layers have been generated, although sometimes there are about 100 more layers still to be added. The mechanism which could account for such growth is yet not understood.

\section{Unconventional Myelin}

The unmyelinated to myelinated transition can happen along the same axon [20]. For example, in a retina-optic nerve junction the same axons are unmyelinated in the retinal part and myelinated in abretinal part (optic nerve proper). The transi- 
tion segment of this retina-optic nerve junction contains unusually thin and short myelin sheaths, intercalated unmyelinated segments and "bizarre paranodes" [20]. This region has been reported to look strikingly similar to some dysmyelinating conditions. Preterminal internodes in the mammalian brain have been shown to be relatively short [21]. Myelinated axons at the PNS/CNS interface, passing through a transitional region, exhibit transitional nodes of Ranvier where a node gap contains a mixture of Schwann cell processes and astrocytic extensions [22]. Interestingly, dendrites and neuronal perikarya can also be sometimes myelinated, with no apparent node formation [23]. That observation shows that myelination may also occur without node formation.

\subsubsection{Myelin-Producing Cells in the CNS}

Myelin is produced by oligodendrocytes in the CNS and by Schwann cells in the PNS. In fact, at least half the volume of the human brain is constituted by glial cells [24]. These cells - oligodendrocytes, astrocytes, microglia - play an important supportive role for neurons. Oligodendrocytes provide electrical insulation to facilitate nerve signal transmission in the CNS, astrocytes maintain metabolic homeostasis for the CNS, and microglia are indispensable for immune surveillance of the CNS. In addition to providing myelin and therefore ensuring rapid nerve conduction, glia are involved in the maintenance of normal axon transport and long-term survival, possibly by supporting the long ensheathed axons metabolically [5].

\section{Historical Overview}

The history of glial research spans more than 150 years. One can assign its starting year to the middle of the $19^{\text {th }}$ century, when the term neuroglia ("Nervenkitt") was first introduced by R. Virchow [25]. He used it to describe a connective substance, which he believed should be present in brain in addition to neurons. In $1865 \mathrm{O}$. Deiters also distinguished cells in the central nervous system that were not neurons; he as well considered them to be the connective tissue cells. Further on, recognition of glial subtypes started, with C. Golgi describing multipolar glial cells and their interaction with blood vessels, followed by S. Ramón y Cajal introducing the term 
astrocyte in the beginning of the $20^{\text {th }}$ century (reviewed in [26]). P. del Rio-Hortega defined oligodendrocytes (which he called interfascicular glia), introduced the term microglia, and started classification of glia [12]. Once glial cells had been properly distinguished from neurons, investigation of their function was launched: according to C. Golgi, glial cells feed neurons; S. Ramón y Cajal proposed that glia insulates nerve fibers; and finally in $1924 \mathrm{~W}$. Penfield suggested the role of oligodendrocytes in formation of myelin [27]. Despite almost a hundred of years of research, which passed since those times, today's neurobiology is still seeking full understanding of glial development and functions [24].

\section{Origin of Oligodendrocytes}

During embryonic development neurons and glia arise from the neuroepithelial cells of the neural tube. In mouse, oligodendrocyte precursors are produced in several waves from both ventral and dorsal areas of neural tube [28]. The initial appearance of oligodendrocyte precursors is restricted to the ventral part of the embryonic spinal cord, where it depends on the adjacent notochord [29]. Notochord produces soluble signals, including Sonic hedgehog (Shh), which induce the local development of floor plate motor neurons (pMN) and of oligodendrocyte precursors [30]. The oligodendrocyte precursors then migrate dorsally and radially to populate developing white matter of the spinal cord. The first wave of oligodendrocyte precursor production both in the spinal cord and in developing brain starts from embryonic date 12.5. At E15, a secondary wave of precursors is generated from more dorsal regions of the spinal cord by trans-differentiation of radial glia. This wave is independent of sonic hedgehog (Shh) signaling, but depends on bone morphogenetic protein (BMP) signaling and fibroblast growth factor (FGF) [28, 31]. There are also ventral and dorsal sources of oligodendrocyte precursors in the forebrain. In the telencephalon, the first wave of precursors is also formed in the ventral areas. Later on, the precursors from the lateral areas are generated, followed by production of the cortex-derived precursors mainly after birth [29].

One possible attractive explanation for the multiple origins of oligodendrocyte precursors is that the ventral source of oligodendrocytes is more "primitive", as they are produced side-by-side with motor neurons of neuronal floor plate (pMN) in 
order to myelinate motor axons and facilitate rapid locomotion [32]. Interestingly, the dorsal wave seem to be evolutionarily more recent, as a dorsal source of oligodendrocytes was found in the telencephalon of the mouse but not of the chick. This extra source of oligodendrocytes might have been acquired by mammals in order to support cortical development, since mammals have a greatly increased cortical volume [29]. Although oligodendrocytes have multiple origins, they seem to have similar molecular and functional properties. At least dorsal and ventral populations are able to substitute functionally for one another, as shown by targeted expression of Diphtheria toxin in the lab of W. D. Richardson [33].

\section{Oligodendroglial Lineage}

Myelination requires a number of differentiation steps in the oligodendroglial cell lineage, accompanied by coordinated change in expression of cell surface markers and morphological reorganization [34]. A totipotent neuroepithelial stem cell gives rise to a glial-restricted precursor cell, which in turn develops into an oligodendrocyte type2 astrocyte (O-2A) progenitor, also called an oligodendrocyte precursor cell (OPC) [35]. O-2A/OPCs are the early proliferative and migratory bipolar oligodendroglial precursors, exposing specific markers: glycolipids GD3 and a chondroitin sulfate proteoglycan NG2. O-2A cells are bipotential, as they can differentiate into either a specific type of astrocytes or oligodendrocytes, depending on culture conditions [36]. Lineage progression then proceeds through the pro-oligodendroblast (pro-OL) stage characterized by multipolar, postmigratory, proliferative cells identified by exposing lipids sulfogalactosylceramides on their surface (sGalC, described in Section 1.2.1). This stage is followed by a transient developmental stage, pre-oligodendrocyte (preGalC), which is also not yet immunoreactive to anti-GalC antibodies (O1) [37].

The onset of terminal differentiation (immature oliodendrocyte stage) is identified by the synthesis and transport to the surface of GalC, expression of CNPase and loss of GD3 and NG2 antigens. After a characteristic lag of one or two days, non-myelinating mature oligodendrocytes develop with the regulated expression of terminal markers myelin-associated glycoprotein (MAG), myelin basic protein (MBP), proteolipid protein (PLP). The presence of myelin oligodendrocyte 
glycoprotein (MOG) correlates with late stages of maturation (myelinating mature oligodendrocyte) [12].

A population of developed oligodendrocytes is not uniform. Depending on their morphology, different types of oligodendrocytes can be distinguished: P. del RioHortega grouped oligodendrocytes in four categories, according to the number of their processes; A. M. Butt et al. also classified four types of oligodendrocytes (from small cells supporting multiple thin axons with thin myelin sheath through intermediate types to the largest cells forming the long, thick myelin sheath) [38]; S. Mori and C. P. Leblond distinguished three types of oligodendrocytes according to the density of their cytoplasm, suggesting that oligodendrocytes become progressively dark as they mature [12].

\subsection{Molecular Composition of Myelin}

The insulating properties of the myelin sheath are due not only to it structure, but also to its molecular composition, low water content and richness in lipids. Myelin is indeed a poorly hydrated structure containing only $40 \%$ water, in contrast to gray matter, containing approx. $80 \%$ water [2]. Myelin dry mass contains a high amount of lipids (approx. 70\%) and low proportion of protein (30\%). This lipid-to-protein ratio is characteristic of the myelin membrane, and it is generally reversed in other cellular membranes [12].

\subsubsection{Lipid Composition of Myelin}

Central nervous system myelin is enriched in certain lipids, although no specific lipids were found to be exclusively present either in myelin, or in non-myelin compartments [2]. Nevertheless, lipid composition of myelin is very characteristic. Myelin is highly enriched in glycosphingolipids, in particular galactocerebrosides, i.e. galactosylceramides (GalC) and sulfogalactosylceramides (sulfated derivatives of galactosylceramides, sulfatides) [39].

Another distinguishing feature of myelin lipid composition is the abundance of plasmalogens, which make up some $80-90 \%$ of the myelin membrane phospholipids. 
In addition to an ethanolamine head group and a long-chain fatty acid attached to the same glycerol phosphate backbone used for phospholipids, plasmalogens contain an unusual fatty alcohol that is attached through an ether linkage [1]. The first reactions in the formation of plasmalogens are catalysed within peroxisomes. Deficiency of plasmalogens causes profound abnormalities in the myelination of nerve cells, which is one reason why many peroxisomal disorders lead to neurological disease $[40,41]$.

Cholesterol is an essential structural component of the plasma membrane of mammalian cells. It accounts for approx. $20-25 \%$ of the lipid molecules in the plasma membrane of most cells, and it is enriched in myelin where it constitutes approx. $28 \%$ of the total lipid weight [42]. In the myelin sheath, cholesterol together with sphingolipids might contribute to the electrical insulator function of myelin, reducing ion permeability of the myelin membrane by reducing the water accessibility to the hydrophobic core [42].

Taken together, these three lipid groups - galactocerebrosides, cholesterol and the plasmalogen form of ethanolamine phosphoglyceride - comprise about $65 \%$ of the total myelin lipids [43]. Lipid composition of myelin and its fractions from various species has been extensively studied since the middle of the last century [44-46]. The summary of the lipid composition of the CNS myelin is listed in a Table 1.1, adapted from [2] (figures in percent of total lipid weight).

Table 1.1: Comparison of lipid composition of CNS myelin and brain

\begin{tabular}{lrrrrrrr}
\hline & \multicolumn{2}{c}{ Myelin } & \multicolumn{2}{c}{ White matter } & Gray matter & Brain \\
\hline \hline Lipid & Human & Bovine & Rat & Human & Bovine & Human & Rat \\
\hline \hline Cholesterol & 27.7 & 28.1 & 27.3 & 27.5 & 23.6 & 22.0 & 23.0 \\
Cerebroside & 22.7 & 24.0 & 23.7 & 19.8 & 22.5 & 5.4 & 14.6 \\
Sulfatide & 3.8 & 3.6 & 7.1 & 5.4 & 5.0 & 1.7 & 4.8 \\
Total galactolipid & 27.5 & 29.3 & 31.5 & 26.4 & 28.6 & 7.3 & 21.3 \\
Ethanolamine phosphatides & 15.6 & 17.4 & 16.7 & 14.9 & 13.6 & 22.7 & 19.8 \\
Lecithin & 11.2 & 10.9 & 11.3 & 12.8 & 12.9 & 26.7 & 22.0 \\
Sphingomyelin & 7.9 & 7.1 & 3.2 & 7.7 & 6.7 & 6.9 & 3.8 \\
Phosphatidylserine & 4.8 & 6.5 & 7.0 & 7.9 & 11.4 & 8.7 & 7.2 \\
Phosphatidylinositol & 0.6 & 0.8 & 1.2 & 0.9 & 0.9 & 2.7 & 2.4 \\
Plasmalogens & 12.3 & 14.1 & 14.1 & 11.2 & 12.2 & 8.8 & 11.6 \\
Total phospholipid & 43.1 & 43.0 & 44.0 & 45.9 & 46.3 & 69.5 & 57.6 \\
\hline
\end{tabular}


With respect to the fatty acid composition, myelin lipids contain a lower proportion of polyunsaturated fatty acids (one-fifth the molar proportion of the gray matter). On the other hand, myelin lipids contain fatty acids of longer chain length (the molar proportion of myelin lipids with C19 - C26 acyl chains is 10 times their molar proportions in gray matter) [44]. These characteristics of the myelin lipids are thought to contribute to myelin membrane organization and its extreme stability, while (1) more saturated hydrocarbon chains are packed tighter in a membrane than polyunsaturated ones due to their configuration, (2) longer hydrocarbon chains provide stronger hydrophobic interactions between the lipids with longer-chain fatty acids, in comparison to the shorter ones [44].

\section{Sphingolipids: Galactosylceramide and Sulfogalactosylceramide}

Sphingolipids are a versatile class of membrane lipids which provide mechanical stability to membranes, constitute ordered membrane domains, and mediate recognition and signaling [47]. Glycosphingolipids are composed of a ceramide backbone and a sugar headgroup. The ceramide backbone consists of a sphingoid base, amidelinked through its amino group to a fatty acid. This hydrophobic ceramide is inserted in a membrane, whereas the sugar headgroup mostly faces the non-cytosolic space [48].

The first step of glycosphingolipid biosynthesis is the condensation of serine and palmitoyl-CoA to form 3-ketosphinganine. Subsequent acylation by the enzyme ceramide synthase produces ceramide. At this stage, unsaturation or hydroxilation is most likely to occur on the cytosolic side of the ER [49]. Ceramide can spontaneously cross the ER membrane and be converted into galactosyceramide (GalC). For this, the transfer of a galactose (Gal) residue from UDP-Gal to ceramide is catalysed by the UDP-Gal:ceramide galactosyltransferase (CGT) [50], which has its active center in the ER lumen [51]. To generate sulfogalactosylceramide (sulfatide sGalC), GalC is sulfated to $\mathrm{HO}_{3} \mathrm{~S}-3 \mathrm{GalC}$ in the lumen of the Golgi apparatus [48].

Sphingolipids are enriched in the outer leaflet of the plasma membrane bilayer, providing mechanical stability. Ceramide-based lipids can self-aggregate in cellular membranes to form a separate phase, which is less fluid than the bulk of liquiddisordered phosphoipids based on diacylglycerol, being one of the bases of "lipid 
rafts" [49]. Sphingolipids also play critical roles in signal transduction (e.g. integrin signaling [52], PKC activity [53]), protein sorting (e.g. to apical domains in epithelial cells [54]) and cell recognition (e.g. blood group antigens, targets for pathogens like Cholera toxin [55]). Several studies provide indications that myelin glycosphingolipids can receive extracellular signals and transmit them across the membrane to the cytosol and the cytoskeleton [56].

The roles of galactosylceramide and sulfatide in myelin functionality are being intensively investigated in studies using transgenic mice deficient for the enzymes of the biosynthetic pathway of galactosphingolipids. In UDP-Gal:ceramide galactosyltransferase (CGT) null mice, CNS and PNS myelin are completely depleted of GalC and its derivative sGalC $[57,58]$. Surprisingly, myelin of CGT-deficient mice contained glucosylcerebroside (a lipid not previously identified in myelin) and had only slight ultrastructural abnormalities $[58,59]$. These mice showed abnormal node of Ranvier formations in the CNS, which explains their tremoring phenotype [60]. This data indicates that GalC and sulfatide are involved in axo-glial interactions and are required for proper CNS node and paranode formation.

Strong reduction of levels of ceramide species with very long fatty acid residues $(\geq \mathrm{C} 22)$ in the brain (also in liver and kidney) is achieved in transgenic mice lacking isoform 2 of the ceramide synthase gene. In these mice, myelin stainability is progressively lost, accompanied by $50 \%$ loss of compacted myelin and $80 \%$ loss of myelin basic protein (MBP) [61].

In human patients, abnormal degradation galactosylceramide and sulfatide leads to two classical genetic myelin disorders: globoid cell leukodystrophy (Krabbe's disease) and metachromatic leukodystrophy (MLD) [4]. The cause of the Krabbe's disease is deficient activity of a lysosomal enzyme galactocerebroside $\beta$-galactosidase which normally cleaves GalC to ceramide and galactose. In this disease, GalC does not accumulate abnormally. On the contrary, it is almost always reduced because of a rapid and almost complete destruction of oligodendroglia and the consequent early cessation of myelination. On the other hand, a toxic metabolite, galactosylsphingosine, does accumulate and appears to be responsible for the devastating pathology of the disease, which typically occurs among infants and takes a rapidly fatal course [62]. MLD is caused by a deficiency of the enzyme arylsulfatase A, lead- 
ing to accumulation of sulfatides in many tissues of the body, causing progressive demyelination, which results in severe, finally lethal, neurologic symptoms [63].

\section{Cholesterol}

Cholesterol is an essential component of mammalian cell membranes and is required for proper membrane permeability, fluidity, organelle identity and protein function [64]. Cholesterol comprises approximately $10 \%$ of the dry weight of a brain, in contrast to other organs, where it represents only about $1 \%$ of the dry weight [65]. Furthermore, $70 \%$ of the cholesterol in the whole brain is incorporated into myelin [66]. There are two major mechanisms that can provide brain cells with sterols: endogenous synthesis from acetyl-CoA (a complex process involving more than 30 enzymes, with 3-hydroxy-3-methyl-glutaryl-CoA (HMG-CoA) reductase being the rate-limiting enzyme) and delivery via circulating lipoproteins [67].

Although brain ability to synthesize cholesterol has been demonstrated, the cholesterol synthesis rate is $20-80$ fold lower in brain than in liver, where it is synthesized at the highest rate [68]. Nevertheless, no movement of cholesterol from plasma lipoproteins across endothelial cells of the blood-brain barrier to the cells of the CNS could be demonstrated. Therefore, de novo synthesis must account for all cholesterol accretion and turnover in the CNS [67]. However, disruption of cholesterol biosynthesis in oligodendrocytes reduced myelin cholesterol content only to $70 \%$ of the cholesterol content of wild-type myelin, indicating that oligodendrocytes can employ cholesterol uptake for myelin synthesis (possibly by horizontal transfer of cholesterol from other brain cells, astrocytes or microglia) [69].

Cholesterol is implicated in myelin biogenesis, as mice deficient for cholesterol synthesis in oligodendrocytes (squalene synthase knock-out) show perturbed myelination, ataxia and tremor [69]. As an essential component of detergent-resistant membrane complexes (hypothesized to form lipid rafts), cholesterol might be involved in ensuring myelin membrane domain formation [70]. Disturbance of cholesterol homeostasis in brain is associated with a number of neurodegenerative diseases, such as familial Alzheimer's disease, in which gene for apolipoprotein E can be involved [71]. Cholesterol deficiency is observed in Smith-Lemli-Opitz-syndrome and desmosterolosis. In these human diseases, characterized by myelination defects and 
complex craniofacial malformations, the last steps of cholesterol biosynthesis are affected [42].

\subsubsection{Myelin Proteins}

Myelin proteins comprise only $30 \%$ of dry weight of myelin. Protein composition of the CNS myelin is simpler than that of the other brain membranes, with two major myelin low-molecular-weight proteins MBP and PLP (and its isoform DM20) constituting $80 \%$ of the total proteins $[2,4,12]$. Although recent proteomics data suggests that MBP and PLP abundance in myelin might have previously been overestimated, these proteins nevertheless remain the major constituents of the myelin proteome [72]. The results of diffraction studies on the structural organization of lipids, proteins and water in the myelin membrane suggest that proteins might play a role in membrane packing and phase separation [73].

\section{Myelin Basic Protein (MBP)}

Myelin basic protein (MBP), which constitutes about 30\% of total myelin proteins, is the second most abundant protein in CNS myelin. It is mainly a peripheral membrane protein, which is present at the cytoplasmic surfaces of the myelin leaflets (corresponding to the major dense line) and is responsible for adhesion of the cytosolic surfaces of multilayered compact myelin $[2,4]$.

In fact, MBP is a family of proteins generated by alternative splicing from the MBP gene, which consists of 7 exons and is contained within a larger transcription unit, called the Golli-MBP. The major MBP isoforms are 21.5, 18.5, 17 and $14 \mathrm{kDa}$ in mouse (with 18.5 and $14 \mathrm{kDa}$ isoforms, which are lacking exon 2, being predominant in adult animals, i.e. constituting approx. 95\% of total MBP) and 21.5, 20.2, 18.5 and $17.2 \mathrm{kDa}$ in humans (with 18.5 and 17.2 isoforms, which are deficient of exon 2 as well, being predominant in adulthood) $[12,74]$.

MBP is an extremely basic and "intrinsically disordered" protein, which is thought to adapt its proper confirmation through binding to negatively charged lipids or interaction with polyanionic proteins, such as actin, tubulin, $\mathrm{Ca}^{2+}$-calmodulin, and clathrin. MBP is known to undergo post-translational modifications, such as gly- 
cosylation, methylation, phosphorylation, etc., which could affect its conformation, interactions with the membrane, and regulate its multifunctionality [74].

The importance of myelin basic protein for the CNS myelin formation is emphasized by the severe phenotype of the Shiverer mice, a naturally occurring mutant with a large portion of the MBP gene deleted [75]. It lacks most compact myelin in the CNS (but not in the PNS), and its CNS myelin lamellae are poorly formed and are lacking the major dense line, indicating a role of MBP in myelin compaction [76, 77]. These mice suffer from severe hypomyelination, generalized tremor, seizures, and die by the end of the myelination period [75].

\section{Proteolipid Protein (PLP)}

PLP is a transmembrane protein (MW around $25 \mathrm{kDa}$ ), comprising together with its splice variant DM20 (MW about $20.5 \mathrm{kDa}$, predominant during development) approx. 50\% of CNS myelin proteins. Proteolipid proteins (lipid-protein complexes) were identified in myelin by J. Folch and M. Lees in 1951 due to their extractability by organic solvent mixtures. PLP is indeed highly acylated (containing 3-4\% (w/w) of covalently bound fatty acids [78]), contributing to its hydrophobic properties. PLP comprises 4 transmembrane $\alpha$-helices, its $\mathrm{N}$ - and C-termini are both localized intracellularly [79].

Numerous functions have been proposed for PLP and DM20 in the CNS, including membrane adhesion and compaction of myelin, formation of the myelin intraperiod line, maturation of oligodendrocytes, etc. [80].

A mutation in the PLP gene (causing abnormality of PLP folding) leads to severe myelin deficiency in the CNS of Jimpy mouse [81]. However, mutant mice that lack expression of a PLP gene fail to exhibit the known dysmyelinated phenotype [82]. Ultrastructurally, myelin lacking PLP shows condensation of the intraperiodic lines correlating with a reduced physical stability, suggesting a role of PLP in formation of intraperiodic line and stabilizing myelin, similar to a "zipper" [83]. Myelination seems also to be sensitive to PLP gene dosage. Transgenic mice carrying extra copies of the PLP gene (providing a valid model of PMD/SPG human diseases, see further) display a phenotype varying from severe, lethal dysmyelination through late-onset demyelination $[84,85]$. In humans, mutations of the X-chromosome linked 
PLP gene cause the heterogeneous syndromes (e.g. severe impairment of motor development and premature death) of Pelizaeus-Merzbacher disease (PMD) and spastic paraplegia (SPG) type 2 (reviewed in $[80,81]$ )

\section{Myelin-Associated Glycoprotein (MAG)}

MAG (MW approx. $100 \mathrm{kDa}$ ) is quantitatively a minor constituent of myelin, representing approx. $1 \%$ of the total CNS myelin protein content. It has a single membrane-spanning domain, its extracellular region contains 5 immunoglobulin-like domains, and it is heavily glycosylated (carbohydrates constitute $30 \%$ of its weight) [86]. Two MAG isoforms have been identified: large MAG (L-MAG) and small MAG (S-MAG), which are generated though alternative splicing and differ in the extent of their cytoplasmic segment [87]. L-MAG expression predominates during myelination process, whereas S-MAG accumulates later on in adult myelin [88, 89]. MAG is a cell adhesion protein that is expressed upon first contact of oligodendrocyte with axon [86]. In a mature myelin sheath this transmembrane protein resides in noncompact myelin, predominantly periaxonally [90], suggesting its role in establishing and maintaining interaction between myelinating glial cells and axons.

MAG has been implicated in myelinogenesis through its participating in gliaaxon adhesion (e.g. due to its ability to bind oligosaccharides on both glycoproteins and gangliosides [91]) and in signal transduction (e.g. through non-receptor tyrosine kinase Fyn [92]). It is also involved in inhibition of axonal outgrowth in the adult CNS (by acting as an additional ligand for the Nogo receptor [93]). Interestingly, soluble MAG, which is physiologically associated with myelin breakdown, seems to induce inflammatory microglial response in culture [94].

Mice deficient in MAG show mild neurological deficits, delay in CNS myelin formation and partially impaired organization of the periaxonal region [95, 96], supporting the view that MAG is involved in recognition between oligodendrocyte processes and axons. 


\section{Myelin Oligodendrocyte Glycoprotein (MOG)}

MOG is a quantitatively minor component of the myelin sheaths. It is a $26-28 \mathrm{kDa}$ glycoprotein, which is specifically expressed in oligodendrocytes starting from the late stages of maturation [97]. MOG is present only in mammalian species. This membrane-spanning protein has a single extracellular immunoglobulin-like domain [97, 98], single glycosylation site, one confirmed transmembrane domain and one more hydrophobic domain of not yet clearly defined membrane topology (possibly positioned within the membrane, so that the C-terminus of MOG is localized in the cytoplasm [99]).

MOG localization in the myelin sheath has not been investigated extensively. A number of reviews confine MOG exclusively to the outermost lamellae of the myelin sheath (i.e. non-compact myelin), although according to the original immuno-EM study, MOG is distributed "preponderantly over the myelin surface associated zone, less in compact myelin and the myelin/axon interface" [100].

Biological function of MOG remains relatively unknown. MOG is an autoantigen, capable of causing experimental autoimmune encephalomyelitis (EAE) and multiple sclerosis (MS). Based on this, MOG was proposed to mediate interactions between myelin and the immune system. It was also suggested, that MOG can also serve as a cellular adhesive molecule, or a regulator of oligodendrocyte microtubule stability [101]. Interestingly, MOG-deficient mice present no clinical or histological abnormalities [102].

\section{2',3'-Cyclic-Nucleotide 3'-Phosphodiesterase (CNPase)}

CNPase represents approx. 2 - 4\% of total myelin proteins. Two CNPase isoforms (48 and $46 \mathrm{kDa}$ ) are generated by alternative splicing from two transcription start sites [103] or by translation from two different translation start sites [104]. CNPase is post-translationally modified, acylated and phosphorylated, and it is associated with the cytoplasmic leaflet of plasma membrane by isoprenylation [105].

CNPase is present in non-compact myelin: in the plasma membrane of oligodendrocytes and their processeses (also during early ensheathment steps), in the outer, inner and paranodal myelin loops, as well as in the incisure-like areas. A cy- 
toplasmic pool of CNPase was also detected in oligodendrocyte perikarya and larger oligodendrocyte processes [106].

The biological function of CNPase is yet not fully understood. Although CNPase posesses enzymatic activity and can hydrolyze artificial substrates (2', 3'-cyclic nucleotides and their 2'-derivatives), its physiological substrate has not been identified. Several lines of evidence suggest CNPase interaction with tubulin and regulation of process outgrowth in oligodendrocytes through cytoskeletal rearrangement [107]. CNPase is involved in maintenance of axon-glia interactions in the CNS, as mice lacking expression of isoform 1 of CNPase have normal myelin assembly and ultrastructure, but display difficulties maintaining integrity of paranodes, develop axonal degeneration and microglial invasion later in life, leading to premature death [108, 109]. CNPase might also play a role of an early expressed regulator of cellular events that culminate in CNS myelination, as oligodendroglial cells overexpressing CNPase appear to mature faster. Furthermore, overexpression of CNPase in transgenic mice leads to aberrant myelination (redundant myelin membrane), lack of myelin compaction concordant with the failure of the cytoplasmic leaflets of compact myelin to fuse [110].

\section{Other Proteins of the CNS Myelin}

A large fraction of myelin proteins is contributed by tetraspans, transmembrane proteins with four membrane-spanning domains (e.g. PLP, MAL, OSP/claudin-11, Cx32). A number of neurological disorders, such as PMD, SPG2, CMT type X, are caused by alteration in gene dosage and mutations in tetraspans genes. The functions of the tetraspans are not completely understood, but seem to include formation of membrane junctions and regulation of growth and migration of myelinating glial cells [111].

A tight junction component, oligodendrocyte-specific protein (OSP/claudin-11, $22-26 \mathrm{kDa})$, contributes $7 \%$ of total myelin proteins [112], and is also expressed in Sertoli cells of the testis. Using double knock-out mice, it was demonstrated that tetraspans OSP/claudin-11 and PLP have essential structural functions in maintaining normal compact myelin and there is redundancy in their functions [113]. 
Connexin 32 (Cx32) belongs to a large multigene family of connexins, which are proteins that form gap junctions (pores allowing small molecules $<1000$ Da to pass between cells) [114].

Myelin and lymphocyte protein (MAL), a $17 \mathrm{kDa}$ protein, which was originally isolated from lymphocytes and was found later in compact myelin [115], is a lipid raft component [116], proposed to be involved in apical sorting in the trans-Golgi network [117].

Apart from tetraspans, relatively small and extremely basic proteins are enriched in the myelin sheath. In addition to MBP, a myelin-associated/oligodendrocyte basic protein (MOBP) was identified as the third most abundant protein in CNS myelin. It is represented by several isoforms $(8.2,9.7$, and $11.7 \mathrm{kDa})$ and might play a similar role to MBP in myelin compaction [118]. MOBPs are located in the major dense line of myelin, no transmembrane domains were predicted in their structure, and it was suggested that topologically MOBP could behave as an intracellular adhesion molecule binding to acidic phospholipids of the intracellular membranous network [119]. Like MBP, MOBP undergoes mRNA-based transport in order to reach myelin sheath [120].

Summarizing our knowledge about the function of myelin proteins, it is worth noting that even in complete absence of such myelin proteins, as PLP/DM20, MOG, MAL, P0, OSP (claudin-11) and PMP22, significant myelin assembly is possible [69]. The only myelin protein, that has been shown to be indispensable for the assembly of the myelin sheath is MBP. However, gain-of-function mutations in myelin protein genes often cause a hypomyelination phenotype in mice [81].

\subsection{Intracellular Trafficking to the Plasma Membrane}

Formation of myelin sheath in development and its maintenance throughout life requires addition of new membrane. For this, oligodendrocytes adapted common cellular processes of membrane biosynthesis, exocytosis and dynamic membrane recycling. 


\subsubsection{Intracellular Trafficking Pathways}

Coordinated intracellular vesicular and non-vesicular transport are essential for myelin membrane biogenesis. The first part of this Section describes common trafficking pathways, delivering constituents to the plasma membrane of mammalian cells, whereas the second part focuses on the specifics of oligodendroglial transport me-chanisms that have been implicated in myelin growth.

\section{Biosynthetic-Secretory Pathway}

Biosynthesis, modification, sorting and transport of many proteins, lipids, and carbohydrates takes place in the biosynthetic-secretory pathway [121]. This pathway comprises the endoplasmic reticulum (ER), which serves as a site for synthesis of lipids and transmembrane proteins (on membrane-bound ribosomes); the Golgi complex, which receives, processes and sorts lipids and proteins arriving from the ER; and membrane-enclosed transport intermediates, which move between ER and Golgi and from Golgi to plasma membrane [122]. Microtubules play an important role in this pathway, both in organelle positioning and in traffic of the transport intermediates [123].

The main biosynthetic compartment of the cell, the endoplasmic reticulum, is organized as a net-like labyrinth of branching tubules and flattened sacs extending throughout the cytosol. In contrast, the elements of what might be called the central sorting compartment, comprising cis- and trans-Golgi apparatus, and the prelysosomal compartment, are normally localized to a juxtanuclear region [124]. Centralizing the newly synthesised components in the Golgi region first and then distributing them to the cell periphery along microtubules might allow selective delivery of the newly made material to the specialized regions of the cell surface.

The endoplasmic reticulum plays a central role in the biosynthesis of lipids and transmembrane proteins. In mammalian cells, import of most proteins into the ER takes place co-translationally. Membrane-bound ribosomes, attached to the cytosolic side of the ER membrane, are engaged in the synthesis of proteins that are being concurrently translocated into the ER [2]. This process involves the signal sequences of the nascent membrane proteins, signal-recognition particles (SRP), SRP receptors 
and translocators (Sec61 complex) in the ER membrane. Some proteins, however, are imported into the ER after their synthesis has been completed, by a mechanism of posttranslational protein translocation (involving BiP). Numerous chaperones reside within the ER lumen (e.g. calnexin, BiP), which help nascent polypeptides to adopt their native confirmation. With the help from lectin chaperones calnexin and calreticulin, the ER also performs "quality control" of protein folding [125]. Catalysis of disulfide (S-S) bond formation (by protein disulfide isomerase), N-glycosylation of proteins (by membrane-bound oligosaccharyl transferase), covalent attachment of a glycosylphosphatidylinositol (GPI) anchor to some membrane proteins take place in the lumen of the ER. Most membrane lipid bilayers are assembled in the ER. In addition to proteins, nearly all of the major classes of lipids, including phospholipids, cholesterol and sphingolipids (ceramide and GalC) are synthesized at the ER membranes [1].

The pathway from the ER to the cell surface goes through the Golgi apparatus (a series of stable compartments, termed the cis-, medial- and trans-Golgi, as well as the trans-Golgi network [126]). Transport vesicles bud from specialized regions of the ER, called ER exit sites, in a sorting process involving the components of the COPII coat. These vesicles fuse into vesicular tubular clusters and move along the microtubules to the Golgi complex, performing the next sorting step on the way, through budding and returning of the COPI-coated vesicles to the ER [127].

In the Golgi apparatus, extensive glycosylation of lipid and protein molecules is carried out [1]. Here, modification of N-linked oligosaccharides added in the ER takes place, as well as O-glycosylation and addition of glycosaminoglycan chains to core proteins to form proteoglycans. Sulfation of the sugars in proteoglycans and of selected tyrosines on proteins also occurs in a late Golgi compartment. Sulfation of GalC, a very abundant myelin lipid (see Section 1.2.1), is also carried out in the Golgi apparatus by sulfotransferases [128]. The trans-Golgi network (TGN) is the major sorting station in the secretory pathway of all eukaryotic cells [129]. Despite extensive studies, the mechanisms of protein and lipid sorting in TGN which contribute to enrichment of specific proteins, as well as sphingolipids and sterols at the plasma membrane are not yet fully understood. It has been demonstrated that this process might involve lipid raft-based mechanisms [130, 131]. 
Budding of the transport vesicles from the trans-Golgi network involves formation of coated pits recruiting adaptor protein AP1 and clathrin. The membrane proteins and the lipids in these vesicles provide new components for the plasma membrane, while the soluble components inside the vesicles are secreted to the extracellular space (e.g. contributing to the formation of extracellular matrix) [1]. On the way from the TGN to the plasma membrane, early/sorting and recycling endosomes can serve as transport intermediates [122, 132].

In fact, one can distinguish two pathways to plasma membrane, diverging in the trans-Golgi network: constitutive secretory pathway, which operates in all cells, and regulated secretory pathway, which is found mainly in cells specialized to secrete products rapidly on demand (e.g. mast cells, neurons). The regulated secretory pathway allows concentration and storage of the secretory material in secretory vesicles (e.g. secretory granules or dense-core vesicles), and release of it by exocytosis in response to an according stimulus (e.g. a chemical messenger, such as a hormone, or, in case of neurons, an electrical excitation) [1]. Constitutive exocytosis pathways which operate in unpolarized cells are likely to have low selectivity. However, polarized cells can selectively direct their plasma membrane proteins to specific plasma membrane domains (see Section 1.3.2), and employ sophisticated sorting mechanisms to achieve this selectivity [133-135].

It is currently thought that most of the intracellular vesicular transport proceeds along microtubules, even in cells which do not possess long cytoplasmic processes and do not require long-distance transport [136]. Commonly, the following scenario is observed: vesicles are carried by plus-end directed kinesins along microtubules toward the cell cortex, and upon reaching the dense cortical actin meshwork the cargo is transferred to myosin Va for delivery to the cell membrane [137].

\section{Exocytosis and its Vesicular Fusion Machinery}

The biosynthetic-secretory pathway enables production, modification and sorting of the plasma membrane components, which are then delivered the cell surface (often to the specific plasma membrane domains) by a process termed exocytosis [1]. The molecular machinery enabling exocytosis and membrane fusion consists of a large variety of proteins that mediate docking (initial recognition of the two membranes) 
and fusion (merging of the two separate lipid membranes into a single continuous bilayer). Despite the complexity of the process and the diversity of the proteins involved, fusion reactions in eukaryotic cells as a rule employ Rabs and SNARE proteins [138-140]. In addition, fusion to plasma membrane often involves a specific protein machinery called the exocyst complex, and may rely on a guidance from lipids, e.g. phosphoinositides [141, 142].

\section{Exocyst complex}

The exocyst is a multisubunit complex involved in docking of exocytic vesicles. It was originally found to be essential for exocytosis in the budding yeast Saccharomyces cerevisiae, as mutations in its components inhibit secretion [143]. The exocyst complex is composed of eight proteins: Sec3p, Sec5p, Sec6p, Sec8p, Sec10p, Sec15p, Exo70p and Exo84p. These proteins are hydrophilic, they interact with each other to form a $19.5 \mathrm{~S}$ complex peripherally associated with the plasma membrane [144]. The exocyst complex localises to regions of active exocytosis and cell surface expansion, including the bud tip in yeast, the vicinity of tight junctions (which is suggested to be an area of active basolateral membrane addition) in epithelial cells [144], growth cones, and the tips of growing neurites in developing neurons [145]. Exocyst is thought to integrate many different inputs in the complex process of exocytosis and to direct vesicles to their precise sites of fusion [141]. Small GTPases including members of the Rab, Rho and Ral families have been shown to associate with the exocyst and to regulate it, although molecular mechanisms of the exocyst regulation and action are not yet fully understood.

\section{Rab proteins}

Regulation of vesicle docking, as well as control of membrane identity, vesicle budding and many other trafficking steps involve Rab proteins [146]. Rab proteins belong to the largest family of monomeric small GTPases, which function as molecular switches that oscillate between GTP- and GDP- bound conformations [147]. Rab GTPases are reversibly associated with membranes by hydrophobic geranylgeranyl groups. This reversible binding to membranes is essential for the regulatory functions of Rabs [148]. In mammals, at least 60 different Rab isoforms have been identified [149], which are distributed to distinct intracellular compartments and regulate transport between organelles. Some Rabs are expressed ubiquitously in hu- 
man tissues whereas others are tissue-specific [140]. Within cells, they are localized to the cytosolic face of distinct intracellular membranes, ensuring identity of cellular membrane compartments [150]. Trafficking steps from the TGN to the plasma membrane have been shown to involve Rab3 and Rab8 [140].

\section{Soluble NSF attachment protein receptors (SNAREs)}

SNARE proteins mediate membrane fusion by bringing the donor and the acceptor membranes in close proximity. Through a zippering-like process, SNAREs assemble into a 4-helix bundle, termed the "core complex", which pulls the two opposed membranes close together, allowing intermixing of the donor and acceptor lipid bilayers $[138,151]$. SNAREs are a superfamily of small proteins (around 100 - 300 amino acids in length) consisting of around 35 distinct isoforms in mammalian cells [149]. They represent a highly conserved class of proteins, characterized by the presence of a SNARE motif, a sequence stretch of 60 - 70 amino acids in length, which arranges in eight amphipathic heptad repeats, which are prone to form coiled coil structures [152]. SNAREs mediate membrane fusion in all trafficking steps of the secretory pathway [139]. Different fusion steps are catalyzed by specific sets of SNARE proteins ("cognate SNAREs") [153, 154]. The following SNARE proteins have been implicated in vesicle fusion with the plasma membrane: Q-SNAREs syntaxins 1 through 4, SNAP-23 and -25, and R-SNAREs VAMP2/synaptobrevin 2, VAMP3/cellubrevin, VAMP7 and VAMP8 [139, 155, 156]. Interaction of specific plasma membrane associated SNAREs with vesicle associated SNAREs mediates merging of membranes during exocytosis. For example, regulated exocytosis can be facilitated through formation of cognate SNARE complexes between plasma membrane associated syntaxin 1A and SNAP-25 and vesicle associated VAMP2/synaptobrevin 2. Syntaxin 4 and SNAP-23 (both plasma membrane associated) and cellubrevin (vesicle associated) can form cognate SNARE complexes which are able to promote constitutive exocytosis [157].

\section{Phosphoinositide PIP2}

The lipid signaling molecule phosphatidylinositol 4,5-bisphosphate (PI(4,5)P2 or PIP2) is specifically enriched in plasma membrane domains. There it can regulate docking and fusion steps during exocytosis and assist invagination of coated pits during endocytosis [142]. Although the precise function of PIP2 in exocytosis 
is uncertain, it is likely that clustering of PIP2 in raft-like domains contributes to establishing docking sites for exocytic vesicles [158, 159]. In regulated exocytosis, PIP2 can be generated at the plasma membrane through the ADP-ribosylation factor (ARF) 6 and calcium-dependent activities of phosphatidylinositol 4-phosphate 5-kinase type I (PIP5 kinase) [160]. Recent data suggests that during neurotransmitter release PIP2 allows binding of synaptotagmin to the membrane thus increasing its calcium sensitivity [161].

\section{Endocytic Pathway}

While the biosynthetic-secretory pathway leads outward from ER towards the Golgi apparatus and cell surface (with a side route leading to lysosomes), the endocytic pathway leads inward from the plasma membrane. In addition to uptake of fluid, solutes and particles, endocytosis results in internalization of the plasma membrane components. There are several types of endocytosis, including clathrin-mediated endocytosis, caveolin-mediated endocytosis, clathrin- and caveolin-independent endocytosis, macropinocytosis and phagocytosis [162].

Clathrin-mediated endocytosis is characterized by formation of clathrincoated endocytic vesicles. Through this mode of endocytosis cells continuously uptake essential nutrients, such as iron-bound transferrin or cholesterol-bound lowdensity lipoprotein (LDL), as well as growth hormones, such as epidermal growth factor (EGF) [163]. Assembly of the clathrin coat [164] involves binding of AP2 adaptor proteins to the cargo receptor, to phosphoinositide PIP2, and to the clathrin molecules. Clathrin triskelions, composed of three clathrin heavy chains and three light chains, assemble into a polygonal lattice, which helps to deform the overlying plasma membrane into a coated pit [165]. Vesicle scission requires the mechanoenzymatic function of GTPase dynamin (which spirals around the neck of the clathrin-coated membrane invagination), actin dynamics, and possibly myosin motor proteins [163, 166].

Caveolin-mediated endocytosis is morphologically defined by a characteristic flask shape of the uncoated plasma membrane invaginations, caveolae (Latin caveolae, "little cavities") [167]. The shape and structural organization of caveolae are conferred by a dimeric protein caveolin. Caveolin resides in cholesterol and 
sphingolipid-rich plasma membrane microdomains (also termed lipid rafts), which can recruit different signaling molecules and membrane transporters. Caveolin binds to cholesterol, inserts as a loop into the inner leaflet of the plasma membrane and self-associates, thereby stabilizing the invagination [168, 169]. For internalization, caveole can employ dynamin [170].

The complex protein machineries that control vesicle budding and fusion, such as SNAREs, Rabs, adaptor proteins, clathrin coats, are significantly conserved between the secretory and the endocytic pathways. Likewise, along with its numerous other functions, the phospholinositide PIP2 is implicated in regulation of both endocytic as well as exocytic steps (involvement of PIP2 in docking/fusion of exocytic vesicles is described in Section 1.3.1) [158]. With respect to endocytosis PIP2 plays an essential role in sequential recruitment of adapter and accessory proteins to endocytic sites. PIP2 binding is also required for the activity and/or membrane recruitment of dynamin (via its PH domain), which is targeted to the neck of endocytic coated pits where it regulates vesicle fission [158]. In addition, association of PIP2 with rafts is implicated in regulation of actin cytoskeleton [171].

After internalization by endocytosis, molecules are sorted and processed in a number of different membrane compartments and organelles, such as early/sorting endosomes, recycling endosomes, multivesicular bodies (MVBs, or late endosomes) and lysosomes [172]. Endosomal compartments, in constant, exchange with each other and additionally interact with the trans-Golgi network [173]. Furthermore, endosomal compartments are shared between the biosynthetic-secretory and endocytic pathways, like the molecular machineries that were described above.

Endocytosed transmembrane proteins can have the following fates: (1) they can be returned to the same plasma membrane domain from which they came (recycling), (2) they can be delivered to a different domain of the plasma membrane (transcytosis), or (3) they can follow the degradation pathway, which leads from the endosomal compartments to lysosomes [1]. In fact, most of the internalized membrane proteins and lipids are returned to the cell surface [172]. 


\subsubsection{Trafficking in Polarized Cells, Specifics of Trafficking in Oligo- dendrocytes}

Cell polarity is a structural and functional subcellular specialization that is ubiquitous in biological systems [174]. A characteristic feature of polarized cells is subdivision of their surface into functionally distinct membrane domains. Myelin can be viewed as an extreme specialization of oligodendroglial plasma membrane. Polarization requires adaptation and specialization of trafficking and sorting machineries which deliver proteins and lipids to the right membrane domains. Here I first introduce highly polarized cells, using epithelial cells and neurons as an example, and then focus on trafficking and membrane recycling in oligodendrocytes.

\section{Common Mechanisms for Establishing Cell Polarity}

Epithelial cells provide a paradigm of cell polarization. A typical epithelial cell has an apical domain, which often carries cilia or microvilli and faces the lumen, and a basolateral domain, which covers the rest of the cell. These two domains have different molecular compositions and are separated by a ring of tight junctions, which prevent proteins and lipids (in the outer leaflet of the lipid bilayer) from diffusing between the domains [135]. The formation of distinct surface domains during epithelial differentiation is accompanied by the reorganization of microtubules, which change their arrangement from radial to parallel, and align along the apico-basolateral polarity axis $[124,133]$. Polarized trafficking machinery delivers membrane components to their destination domain using either a direct route or by transcytosis [175].

A nerve cell is another example of a polarized cell. Its plasma membrane is organized into domains with different function and distinct molecular compositions. In neurons, two broad surface domains separated by an axonal hillock can be distinguished: the plasma membrane of the nerve cell body and dendrites (specialized to receive signals from other nerve cells), and the plasma membrane axon and its nerve terminals (specialized for signaling to other neurons) [1]. Similar to the scenario observed in epithelial cells, both the maintenance and establishment of neuronal polarity involve coordinated regulation of cytoskeleton and membrane trafficking machinery [176]. Vesicles destined for specific domains are pre-sorted in the trans- 
Golgi network. Some membrane proteins reach the axon by transcytosis or are extruded from the unsuitable domains by destructive endocytosis [177].

A number of studies have suggested similarities between the apical and basolateral domains of epithelial cells and the plasma membrane domains of neurons or myelinating glia $[13,130,178,179]$. Yet more work is necessary to clarify the molecular relationship between the domains in these cell types.

\section{Biosynthetic Pathways of the Main Myelin Components}

During its biogenesis, myelin is produced as a specialization of the oligodendrocyte plasma membranes on a remarkable scale of $5-50 \times 10^{3} \mu \mathrm{m}^{2}$ membrane per cell per day [37], compared with a cell body surface area of approximately $300 \mu \mathrm{m}^{2}$ [179]. According to another estimation, an actively myelinating oligodendrocyte synthesizes three times its weight as myelin membranes each day [43]. This remarkable rate of membrane production requires coordinated biosynthesis of myelin proteins and lipids (see Sections 1.2.1, 1.2.2 and 1.3.1), vesicular and mRNA transport (see Sections 1.3.1 and 1.3.1), coordinated exo- and endocytosis (see Sections 1.3.1 and 1.3.1), and cytoskeletal dynamics [180]. Furthermore, the myelin membrane components might need to travel significant distances along an oligodendrocyte processes to reach their target membranes, which imposes additional burden on the biosynthetic and trafficking machineries.

Expression of the main myelin proteins and enzymes for lipid biosynthesis is developmentally regulated. In both mice and rats, accumulation of myelin takes place between postnatal days 12 - 30, correlating with increased expression of myelin protein genes (e.g. maximum level of mRNA coding for MBP was observed around P20) and genes coding for a rate-limiting enzyme of galactosylceramide biosynthesis (e.g. CGT mRNA peak around P20 - P30) [181].

It is widely accepted that the most abundant myelin protein, the proteolipid protein (PLP), is synthesized by oligodendrocytes on ER-bound ribosomes and transported through Golgi to the cell surface [80]. However, a detailed sorting mechanism for PLP transport into respective myelin domains is still lacking. It has been shown that palmitoylation is a sorting determinant for PLP transport to the myelin membrane [182]. There are also indications that PLP sorting involves association of PLP 
with the cholesterol- and sphingolipid-rich membrane domains (lipid rafts) in the Golgi complex of cultured oligodendrocytes [130]. Vesicular co-transport of PLP and cerebroside sulfates has also been shown on microsomal subfractions from rat brain [183]. Inhibition of the synthesis of glycosphingolipids (with L-cycloserine, an inhibitor of the synthesis of sphingosine) was shown to decrease the translocation of PLP (but not of MBP) to the myelin membrane in rat brain slices as well [184]. Other studies demonstrated that sorting and trafficking of PLP to the plasma membrane is not affected upon the absence of lipid and protein sulfation in oligodendrocytes [185], in GalC- and sulfatide-deficient CHO cells [186], and in vivo in mice, unable to synthesise GalC and sGalC [58]. In addition, endocytic recycling seems to be involved in PLP trafficking in primary oligodendrocytes (see Section 1.3.2). Furthermore, indirect transport of PLP to the myelin sheath (via the plasma membrane), rather than vesicular transport from the Golgi complex has also been suggested [130, 179]. Taken together, these findings might indicate transient involvement of multiple pathways in PLP transport, depending on the cell type and developmental stage studied.

The second most abundant myelin protein, MBP, follows a completely different pathway of biosynthesis and transport. Early pulse-chase studies showed that newly synthesised MBP was reaching the myelin compartment within minutes of translation, whereas incorporation of PLP (and some other myelin proteins, e.g. glycoproteins) was 30 - 45 min delayed [187]. After export from the nucleus, MBP mRNA is assembled into RNA granules through binding of the heterogenous nuclear ribonucleoprotein A2 (hnRNP A2) to the RNA trafficking signal localized in the 3' UTR of the MBP mRNA. The granules are transported along microtubules at a velocity $0.2 \mu \mathrm{m} / \mathrm{s}$ using anterograde motor kinesin [188]. MBP mRNA is translated on free ribosomes [189] in the distal processes of oligodendrocytes near the presumptive sites of myelin assembly [190]. Steering MBP expression to the cell periphery may help to prevent possible deleterious adhesive effect of the MBP on intracellular membranes. In addition to MBP mRNA, several other mRNAs, including those coding for MOBP, tau and CAII, are localized to the distal processes of oligodendrocytes (reviewed in [191]). 
The major myelin glycosphingolipid GalC (see Section 1.2.1) is produced in the ER. Ceramide synthesis occurs on the cytosolic side of the ER, galactosylation is carried out in the lumen of ER, followed by sulfation of a fraction of its pool in the Golgi complex (lumenal surface) [49].

Cellular cholesterol (see Section 1.2.1) is also synthesised de novo in the ER, which contains key metabolic enzymes of cholesterol biosynthesis (HMG-CoA reductase and squalene synthase) $[192,193]$.

Being an inherent part of the membranes of transport vesicles, lipids follow the biosynthetic-secretory pathway (described in Section 1.3.1) and are trafficked by vesicular transport to the plasma membrane (described in Section 1.3.1). Alternative non-vesicular transport has been proposed both for sphingolipids (ceramide, glucosylceramide) [194] and cholesterol [193]. A significant fraction of GalC might circumvent the Golgi, to be directly targeted from the ER to endosomal compartments and/or myelin membranes in sigma-1 receptor positive lipid droplets [195]. The non-vesicular transport of cholesterol from the ER to the plasma membrane is facilitated by the soluble lipid transfer proteins (LTPs, e.g. ORP2). By X-ray crystallography it has been shown that LTPs contain a hydrophobic pocket which can bind a sterol molecule [196]. They can interact directly with lipid membranes to extract sterol and then carry it to the acceptor membranes [193].

\section{Exocytic Pathway in Myelin Production}

\section{Sec8}

An exocyst component Sec8 (see Section 1.3.1), has been shown to be important for oligodendrocyte morphological differentiation, oligodendrocyte process growth and arborization [180].

\section{Rabs}

The following Rab proteins are up-regulated during oligodendrocyte maturation: Rab3a (enriched at synaptic vesicles in neurons), Rab5a (implicated in traffic from plasma membrane to early endosomes) and Rab8a (confined to exocytic vesicles) $[156,197]$. Of these, Rab3a seems to be the most promising Rab candidate for regulating vesicular traffic to plasma membrane of oligodendrocytes. When over- 
expressed together with SNAP-29 in HEK293 cells, it enhances surface-directed trafficking of myelin proteolipid protein (PLP) [198].

\section{SNAREs}

Variety of syntaxins and other SNARE proteins have been detected in oligodendrocytes (listed in $[156,199])$. With respect to putative exocytic SNAREs, the following has been described:

Plasma membrane Q-SNAREs syntaxins 3 and 4, SNAP-23 are up-regulated during maturation of oligodendrocytes [199, 200].

Plasma membrane Q-SNARE syntaxin 2 has constant expression in primary oligodendrocytes [199].

Expression of endosomal R-SNARE VAMP3/cellubrevin is increased with oligodendrocyte maturation [199, 200]. Vesicular SNARE VAMP2/synaptobrevin 2 is up-regulated during oligodendroglial maturation according to [200], but shows constant expression along with VAMP7 according to [199].

Enrichment of SNAP-29 in oligodendrocytes during myelination has been reported in [198]. However, decrease of SNAP-29 expression with oligodendroglial maturation has been shown as well [199]. Interaction of SNAP-29 and Rab3a has also been reported [198].

A cognate core SNARE complex composed of VAMP3, syntaxin 4, and SNAP23 might mediate fusion of endosome-derived vesicles with oligodendroglial plasma membrane [199].

Subcellular localization of exocytic SNAREs in oligodendrocytes has been reported as follows: syntaxins 2 - 4, SNAP-23, VAMP2 and VAMP3 show vesicular staining (with VAMP3 being confined to recycling endosomes); SNAP-29 exhibits cytoplasmic staining pattern [199]; syntaxin 3 localizes to the cell body and primary processes, whereas endogenous syntaxin 4 primarily localizes to myelin-like membranes in primary oligodendrocytes [179]. Syntaxins 2-4, SNAP-23 and VAMP7 accumulate in mature myelin [199].

\section{Endocytic Pathway in Myelin Membrane Formation}

Myelination requires rapid mobilization of plasma membrane and polarized membrane growth. The temporal and spatial coordination of this process can be sup- 
ported by endocytic recycling mechanisms. Endocytic recycling has been demonstrated for such myelin proteins as PLP, MAG and MOG [201, 202].

In the absence of neurons, a large fraction of PLP is internalized from the plasma membrane of oligodendroglial cells by a cholesterol-dependent and clathrinindependent endocytosis pathway (requiring actin and the RhoA GTPase) and is stored in late endosomes/lysosomes [201, 202]. Upon maturation, the rate of endocytosis is reduced, and neuronal cAMP-dependent signaling triggers myelin membrane exocytosis from endosomal storage sites [201]. In stably expressing PLP polarized HepG2 cells, PLP can follow a transcytosis-like pathway (from apical to basolateral membrane) [179].

Both MAG and MOG can be endocytosed via a clathrin-dependent pathway [202]. After internalization they follow distinct trafficking routes: MAG (like PLP) is targeted to late endosomes/lysosomes [202, 203], whereas transport of MOG goes through recycling endosome [202].

\section{Oligodendroglial Cytoskeleton}

Cytoskeleton plays important role in intracellular trafficking processes. Microtubules (cylindrical polymers of $\alpha$ - and $\beta$-tubulin protein subunits) and microfilaments (filamentous polymers of actin, F-actin) serve as transport tracks for vesicles, proteins, mRNA granules, and cellular organelles. Cargoes are trafficked along the microtubules and microfilaments by motor proteins including kinesin, dynein, and myosin (detailed in [137]).

Oligodendroglial cytoskeleton contains microtubules and microfilaments but appears to be devoid of intermediate filaments [37, 204]. In cultured oligodendrocytes, microtubules are found in the cell body and main processes, whereas an F-actin network is localized immediately beneath the plasma membrane, extending from the cell body into peripheral branches and thin villi [205]. There are indications that the majority of microtubules in the processes of mature oligodendrocytes are oriented with their plus end to cell periphery [206].

In myelin, cytoplasmic pockets, such as paranodal loops, the inner tongue, and the radial component most probably contain both microtubules and microfilaments, 
as well as organelles [207], whereas cytoskeletal components are likely to be removed from compact myelin [208].

Differentiation, maturation and myelination goes along with tremendous morphological changes and cytoskeletal rearrangements in oligodendrocytes.

During oligodendrocyte process outgrowth, the extracellular matrix (ECM) proteins are involved in signaling cascades leading to cytoskeletal remodeling: laminin 2 binds $\alpha_{6} \beta_{1}$ integrin, leading to phosphorylation of $\beta_{1}$ by integrin-linked kinase (ILK), which in turn mediates actin polymerization through recruitment of paxillin and the N-WASP/Arp2/3 complex [209]. Another ECM protein fibronectin initiates processes outgrowth through a Fyn-kinase-regulated pathway, which can inactivate RhoA, thus allowing actin polymerisation [210], or induce reorganization of microtubules through interaction with the cytoskeletal protein Tau [211].

It is currently not known which cytoskeletal remodeling processes are involved in more advanced stages of myelination and wrapping. It was suggested that compaction might require removal of cytoskeletal components [208]. For this, a tubulin deacetylating protein SIRT2 might promote microtubules retraction [212]. Rearrangements of actin cytoskeleton can be triggered by signaling through sphingolipids, possibly initiated via contact of extracellular membrane leaflets during wrapping [213].

In addition to the described intracellular trafficking processes, dynamic membrane recycling and cytoskeletal rearrangements, myelin membrane biogenesis is likely to involve lateral membrane sorting mechanisms (discussed in the following Sections).

\subsection{Membrane Organization}

\subsubsection{Cell Membrane Models}

Biological membranes are currently viewed as two-dimensional mixtures of a huge number of components, that combine fluidity and order [214]. In the 1970s, S. Singer and G. Nicolson proposed a dogmatic model of membrane structure, a fluid 
mosaic model. The fluid mosaic model predicts random two-dimensional long-range distribution of integral proteins in the lipid bilayer [215].

Within the following years, a substantial amount of evidence had accumulated pointing at lateral heterogeneity in cellular membranes, which commenced in a lipid raft hypotheses [54]. The lipid raft hypothesis assumes that cholesterol and sphingolipids associate with each other to form platforms for segregation of proteins. The selective incorporation of specific proteins and lipids in rafts may be important for membrane trafficking, signaling, assembly of specialized structures (e.g. virus budding), endocytosis and immune response [70, 216]. However, the lipid raft hypothesis is still a matter of controversy due to limited evidence of raft existence in living cells and varieties in detected sizes, protein composition and life-times of rafts. Lipid order and phase separation principles, extensively modeled and studied in vitro underlie the raft hypothesis for biological membranes.

\subsubsection{Phase Separation Concept}

In physics, phase of a substance is defined as "a form of matter that is uniform throughout in chemical composition and physical state". The word phase comes from the Greek word "appearance" [217]. A binary system consisting of two liquids $\mathrm{A}$ and $\mathrm{B}$ remains a single phase if liquid B dissolves in liquid A completely. When more $\mathrm{B}$ is added than can be dissolved in A, the system separates into two phases. These two phases are in equilibrium with each other, the more abundant one consists of A saturated with $\mathrm{B}$, the less abundant is made of $\mathrm{B}$ saturated with $\mathrm{A}$. It is worth noting that as miscibility of components depends on temperature, so does phase separation. Upper critical temperature is the highest temperature at which phase separation occurs. Some systems show lower critical temperature, others have both upper and lower ones [217].

Coexistence of lipid phases has been repeatedly observed in well-defined model membranes (reviewed in [218, 219]). For example, three-component lipid mixtures, composed of cholesterol combined with a lipid with a low melting temperature $(\mathrm{Tm})$ and a high Tm lipid (e.g. cholesterol : phosphatidylcholine (PC) : sphingolipid in an equimolar mixture) eagerly separate into liquid ordered (Lo) and liquid disordered 
(Ld) phases. In such model membranes, the Lo phase covers two-thirds of the surface and is enriched in sphingomyelin (1.5-fold) and cholesterol (4-fold) compared to the Ld phase, which in turn contains a four times higher PC concentration (estimated at $\left.37^{\circ} \mathrm{C}\right)[220]$.

In a cholesterol-dependent lateral segregation, the planarity of the rigid sterol ring favors interaction with straighter, stiffer hydrocarbon chains of saturated lipids and disfavors interaction with the more bulky unsaturated lipid species. Sphingolipids tend to display longer and more saturated hydrocarbon chains, thus favoring interaction with cholesterol. In addition, the strongly hydrated head groups of sphingolipids provide shielding to hydrophobic cholesterol ("umbrella effect"). Interaction with cholesterol forces neighboring hydrocarbon chains into more extended conformations, increasing membrane thickness and promoting segregation further through hydrophobic mismatch. Thus lateral separation yields a thicker Lo and a thinner Ld phase [221].

Studies on model membranes provide numerous evidences of lipid phase separation. Yet, the relationship between these simple model mixtures and biological membranes containing hundreds of different proteins and lipids is not clear. Heterogeneity is one problem: complexity of the proteome aside, the cellular lipidome counts about a thousand different lipids [222]. Another hindrance for determining phase behaviour of more biologically relevant lipid-protein mixtures lies in the disruption of the bilayer phase by increasing the protein or peptide concentration [223].

Protein segregation in membranes is thought to be affected by hydrophobic mismatch. Matching thickness of the hydrophobic region of the lipid bilayer and the length of the hydrophobic region of the proteins has been already proposed in a mattress model of membrane organization in 1984 [224]. In case of phase separation, the protein will select a phase which provides the better match (as mentioned above, Lo is though to be thicker, Ld is thinner) [218]. Thus, phase separation can effect diffusion of membrane components so that a single molecule will visit different phases due to Brownian motion, but it will be transiently trapped in domains with favorable interactions [214]. 
Do lipids indeed determine protein segregation? Is a reverse scenario true, and proteins effect their lipid environment and promote or prevent lipid phase separation? The most reasonable answer is that nonrandom mixing in biological membranes comes from the interaction energies of molecules in contact with each other. Thus, in some mixtures, protein-protein and protein-lipid interactions would dominate the clustering and phase behavior, whereas in other mixtures various lipid-lipid interactions would play a larger role [223].

\subsubsection{Common Mechanisms of Membrane Domain Assembly}

The current school of thought postulates that cellular membranes exhibit lateral heterogeneity. Several cellular and molecular mechanisms could contribute to lateral organization of plasma membrane. Distinct plasma membrane domains can arise from the targeted delivery of appropriate membrane components or from polarized membrane recycling (described in Section 1.3.2). Alternatively, membrane components could be delivered to all regions of the cell surface indiscriminately, followed by selective retention (stabilization in some locations and selective elimination from others). A "horizontal trafficking" hypothesis is currently emerging for describing targeting of membrane proteins to their destination domains. For example, targeting of AMPA receptors can be explained by surface translocation of AMPA receptors from nonsynaptic to synaptic sites ("horizontal trafficking") [225].

Lateral sorting in the plain of a membrane (e.g. in trans-Golgi membranes or in plasma membrane) could be involved in all of these mechanisms.

In the plasma membrane, two main compartmentalizing forces were demonstrated to be at work: phase separation and cytoskeleton-based confinement (meshwork model) [226]. Possibly these two processes are not independent from each other, as both protein and lipid components of rafts (e.g. PIP2) have been shown to communicate with the cytoskeleton directly [227].

Studies of phase separation processes in biological membranes detected lateral inhomogeneities ranging from few nanometers to micrometers. The main methods currently used for investigating membrane dynamics are advanced microscopic techniques such as FRAP, AFM, FSC and SPT. For example, in one of the re- 
cent works, membrane domains were visualized in live biological membranes with STED, and were shown to be approximately $20 \mathrm{~nm}$ size and highly dynamic (dwell period 10 - $20 \mathrm{~ms}$ ) [228]. Existence of large micrometer size domains related to particular functional or structural aspects is considered possible for specialized biological membranes [218]. Macrodomains are found in immunological synapses of activated lymphocytes, in cell-cell adhesion complexes, and in the leading edge of migrating cells [229]. Lipid order as a component of lateral membrane organization can be assessed with a fluorescence probe Laurdan. For example, Laurdan fluorescence microscopy was used to detect condensation of the plasma membrane during T lymphocyte activation [230].

\subsubsection{Specifics of Myelin Membrane Domain Organization}

A mature myelin sheath is a polarized membrane with two broad domains containing asymmetrically distributed proteins: compact and non-compact myelin [13]. In compact myelin membranes are condensed, whereas the non-compact areas comprise cytoplasm. Tight junctions composed of OSP/claudin-11 serve as physical barriers between compact and non-compact myelin, analogous to the belts of tight junctions, separating apical and basolateral membrane domains in epithelial cells [231, 232].

In the compact internodal myelin, proteins MBP and PLP are abundant. The non-compact myelin domains (paranodal loops, inner and outer loops, and cytoplasmic incisures) are differentially enriched in CNPase, MAG (predominantly periaxonal), neurofascin-155 (predominantly paranodal) and Cx32 (localized paranodally and in incisures) [13].

Which lateral compartmentalization mechanisms are involved in myelin polarization? Interestingly, when S. Singer and G. Nicolson discussed applicability of their fluid mosaic model for biological membranes, they suggested that it might not reflect myelin organization, as "myelin may be rather rigid than fluid mosaic structure" [215]. Indeed, myelin membranes differ significantly from the plasma membrane. Myelin is characterized by extremely high lipid content and is unusually enriched in sphingolipids and lipids with long and saturated acyl chains (i.e. more 
condensed or ordered lipids) [44], indicating that lipid-based domain organization might be highly relevant for myelin.

However, due to the complications arising from the multilayer nature of myelin, the majority of studies on myelin microdomains were performed using a biochemical detergent extraction approach. It provided a huge body of data on solubility of myelin proteins, which varied depending on the type of detergent used, the age of experimental animals, temperature and time of incubation. [116, 130, 233]. The functional relevance of partitioning of studied proteins in detergent resistent domains is not yet clarified. In fact, although the original definition of rafts was based on detergent solubility, this technique is presently considered to induce artifacts in membrane structure [233].

A variety of studies compared myelin protein targeting and apical/basolateral sorting in epithelial cells, and consensus on this issue has also not been reached. There are indications that after early biosynthesis PLP localizes at the cell body plasma membrane of oligodendrocytes, similar to the apical viral model protein HA, although in developed oligodendrocytes PLP is found in the sheets [130]. In polarized Madin-Darby canine kidney (MDCK) cells, stably expressed PLP was transported to the apical domain [178]. Similarly, upon transient expression in polarized HepG2 cells, PLP was localized at the apical membrane, although it moved by transcytosis to the basolateral domain when expressed stably [179]. Furthermore, upon ectopic expression in MDCK cells, PLP, MAG and MOG were targeted to distinct domains. MOG was sorted exclusively to MDCK basolateral membrane, PLP was directed to MDCK apical membrane, while MAG did not show targeting preferences within MDCK cells [178]. In epithelial cells, myelin and lymphocyte protein MAL determined apical sorting and transport, resulting in apical surface expansion upon overexpression [117, 234].

Taken together, despite extensive data on myelin biogenesis accumulated so far, further studies are necessary to resolve the mechanisms of myelin lateral organization. 


\subsection{Aims and Objectives}

Myelin insulation facilitates rapid nerve conduction and provides axon support in higher vertebrates. During myelination, vast amounts of myelin membrane are produced by oligodendrocytes, requiring coordinated performance of oligodendroglial biosynthetic, transport and sorting machineries. Myelin membranes are expanding, wrapping around axons and undergoing compaction fairly simultaneously. A mechanism which could account for this fascinating mode of myelin growth has not yet been found. Therefore, one aim of this study was to elucidate cellular machinery enabling myelin membrane expansion and obtain insights into the mechanisms underlying myelin growth. For this, we sought to identify sites of synthesis, transport and plasma membrane insertion of myelin components in a model cell culture system, using immunocytochemistry, overexpression analysis, live imaging and biochemical assays.

During its biogenesis, myelin membranes acquire polarized domain organization, which is crucial for saltatory nerve conduction. Since myelin is highly enriched in lipids, especially sphingolipids and cholesterol, the lateral lipid-based sorting mechanisms are likely to be involved in the assembly of myelin membrane domains. These putative sorting processes in myelin are presently not fully understood, therefore the second aim of this work is to reveal the role of lateral sorting in molecular organization of myelin membranes. To this end, we sought to characterize the molecular composition and properties of myelin domains and determine the contribution of their constituents in possible self-organization processes. For depletion experiments, both primary cell cultures and in vivo mouse models were chosen for microscopic and biophysical analysis, in conjunction with in vitro reconstitution assays. 


\section{Chapter 2}

\section{Materials \& Methods}

\subsection{Materials}

\subsubsection{Chemicals and Consumables}

All chemicals were obtained from Sigma-Aldrich (Sigma-Aldrich Chemie GmbH, Munich, Germany), Merck (Merck KGaA, Darmstadt, Germany), or AppliChem (AppliChem GmbH, Darmstadt, Germany), unless stated otherwise.

All basal media, supplements, antibiotics and sera for cell culture were purchased from Gibco/Invitrogen (Invitrogen GmbH, Darmstadt, Germany), unless specified.

Consumables were purchased from Falcon (Becton Dickinson Labware Europe, Le Pont De Claix, France) and Eppendorf (Eppendorf AG, Hamburg, Germany). CELLSTAR culture vessels were obtained from Greiner Bio-One (Greiner Bio-One GmbH, Frickenhausen, Germany).

\subsubsection{Antibodies}

Primary antibodies used in this study are listed in Table 2.1. Secondary fluorophoreand peroxidase-conjugated antibodies were purchased from Dianova (Hamburg, Germany). 
Table 2.1: Antibodies

\begin{tabular}{|c|c|c|c|}
\hline Target & Host species & Application & Reference \\
\hline Actin (AC-40) & Mouse IgG2a & WB (1:500) & Sigma-Aldrich, Munich, Germany \\
\hline Adaptor protein AP50 & Mouse IgG1 & IF $(1: 100)$, WB $(1: 250)$ & BD Transd. Lab., San Jose, CA, USA \\
\hline Akt (pan and phospho) & Rabbit & IF $(1: 200)$ & Cell Signaling, Danvers, MA, USA \\
\hline Calnexin & Rabbit & IF $(1: 200)$, WB $(1: 1000)$ & Stressgen Bioreag., Victoria, Canada \\
\hline Caveolin & Mouse IgM & IF $(1: 200)$, WB $(1: 500)$ & BD Transd. Lab., San Jose, CA, USA \\
\hline Clathrin heavy chain & Mouse IgG1 & IF $(1: 200)$, WB $(1: 1000)$ & BD Transd. Lab., San Jose, CA, USA \\
\hline GalC (clone MAB342) & Mouse IgG1 & IF $(1: 200)$ & Millipore, Billerica, MA, USA \\
\hline GFAP & Rabbit & IF $(1: 100)$, WB $(1: 1000)$ & Promega, Mannheim, Germany \\
\hline GFP & Rabbit & IF $(1: 100)$, WB $(1: 1000)$ & Abcam plc, Cambridge, UK \\
\hline GM130 (cis-Golgi) & Mouse IgG1 & IF $(1: 200)$, WB $(1: 500)$ & BD Transd. Lab., San Jose, CA, USA \\
\hline Golgi 58K (trans-Golgi) & Mouse IgG1 & IF $(1: 100)$, WB $(1: 1000)$ & Sigma-Aldrich, Munich, Germany \\
\hline HA & Rabbit & IF $(1: 400)$, WB $(1: 1000)$ & Abcam plc, Cambridge, UK \\
\hline HA.11 & Mouse IgG1 & IF $(1: 1000)$, WB $(1: 1000)$ & Covance, Berkeley, CA, USA \\
\hline Iba1 & Rabbit & IF (1:200), WB (1:2000) & Wako Chem. GmbH, Neuss, Germany \\
\hline Lamp-1 & Rat IgG2a & WB $(1: 1000)$ & Santa Cruz Biot., CA, USA \\
\hline Lamp-1 & Rat IgG2a & IF $(1: 200)$ & BD Biosciences, San Jose, CA, USA \\
\hline MAG (clone 513) & Mouse IgG1 & IF $(1: 200)$, WB $(1: 500)$ & Millipore, Billerica, MA, USA \\
\hline MAG (clone 513) & Mouse IgG1 & WB (1:500) & self-produced, hybridoma SN [235] \\
\hline MBP & Mouse IgG1 & IF $(1: 1000)$ & Sternberger, Lutherville, MD, USA \\
\hline MBP & Rabbit & IF $(1: 300)$, WB $(1: 2000)$ & DakoCytomat., Carpinteria, CA, USA \\
\hline MOG (clone 8-18-C5) & Mouse IgG1 & IF (1:100), WB (1:500) & Millipore, Billerica, MA, USA \\
\hline Myc & Rabbit IgG & IF $(1: 500)$, WB $(1: 1000)$ & Upstate, Lake Placid, NY, USA \\
\hline Myc (clone 9E10) & Mouse IgG1 & IF $(1: 400)$ & Sigma-Aldrich, Munich, Germany \\
\hline Myc & Mouse IgG2a & WB $(1: 1000)$ & Cell Signaling, Danvers, MA, USA \\
\hline $\mathrm{O} 1$ & Mouse IgM & IF $(1: 50)$ & {$[39,236]$} \\
\hline $\mathrm{O} 4$ & Mouse IgM & IF $(1: 50)$ & {$[39,236,237]$} \\
\hline PIP2 & Mouse IgG2b & IF $(1: 100)$ & Assay design, Ann Arbor, MI, USA \\
\hline PLP (O10) & Mouse IgM & IF $(1: 10$, live $)$ & {$[236,238]$} \\
\hline PLP (clone 3F4) & Mouse IgG & WB $(1: 100)$ & [239] \\
\hline PLP (P6) & Rabbit & IF $(1: 300)$ & {$[240]$} \\
\hline Rab 3 & Mouse IgG1 & IF $(1: 100)$ & Synaptic Sys., Göttingen, Germany \\
\hline $\operatorname{Sec} 6$ & Mouse IgG & IF $(1: 200)$ & Merck KGaA, Darmstadt, Germany \\
\hline Synaptophysin 1 & Mouse IgG & WB $(1: 1000)$ & Synaptic Sys., Göttingen, Germany \\
\hline Syntaxin 1 & Mouse IgG & IF $(1: 100)$ & Synaptic Sys., Göttingen, Germany \\
\hline Syntaxin 2 & Rabbit & IF $(1: 100)$ & Synaptic Sys., Göttingen, Germany \\
\hline Syntaxin 3 & Rabbit & IF $(1: 100)$ & Synaptic Sys., Göttingen, Germany \\
\hline Syntaxin 4 & Rabbit & IF $(1: 100)$ & Synaptic Sys., Göttingen, Germany \\
\hline Synaptobrevin/Vamp 2 & Mouse IgG & IF $(1: 200)$ & Synaptic Sys., Göttingen, Germany \\
\hline SNAP 23 & Rabbit & IF $(1: 100)$ & Synaptic Sys., Göttingen, Germany \\
\hline Tubulin, $\alpha$ or $\beta$ & Mouse IgG1 & IF $(1: 2000)$, WB $(1: 5000)$ & Sigma-Aldrich, Munich, Germany \\
\hline Tubulin, $\beta$ III & Mouse IgG1 & IF $(1: 3000)$ & Promega, Mannheim, Germany \\
\hline Tubulin, polyglut. & Mouse IgM & IF $(1: 1000)$ & Sigma-Aldrich, Munich, Germany \\
\hline VSVG & Rabbit & IF $(1: 500)$, WB $(1: 2000)$ & Sigma-Aldrich, Munich, Germany \\
\hline
\end{tabular}




\subsubsection{DNA Constructs}

Vectors, commercial and previously published plasmids are listed in Table 2.2.

Table 2.2: DNA constructs, acquired

\begin{tabular}{lll}
\hline Construct & Description/Application & Reference \\
\hline \hline MOG full length & subcloning & {$[241]$} \\
pßactin-GFP-S-MAG & subcloning & {$[115]$} \\
pcDNA3-Flag1-td-EosFP & expression of photoconvertible FP & MoBiTec GmbH, Göttingen, Germany \\
pcDNA3.1(-) & vector for mammalian expression & Invitrogen, Carlsbad, CA, USA \\
pDsRed2-C1 & expression of RFP in mammalian cells & Invitrogen, Carlsbad, CA, USA \\
pHluorin-synaptobrevin & subcloning for TIRF & {$[242]$, a gift from Prof. G. Miesenböck } \\
PLP-myc-EGFP & subcloning & {$[201]$} \\
pmaxGFP & expression of GFP in mammalian cells & Lonza Group Ltd, Basel, Switzerland \\
pSFV-Helper2 & helper vector for SFV assembly & {$[243,244]$} \\
pSFV2gen & alphaviral vector & {$[243]$} \\
VSVG3-SP-YFP & subcloning into alphaviral vector & P. Keller based on VSVG3-FP [245] \\
\hline
\end{tabular}

Constructs generated in this study are listed in Table 2.3. Cloning strategies and sequences of the constructs are documented in Appendix A.

Table 2.3: DNA constructs, cloned

\begin{tabular}{ll}
\hline Construct & Description \\
\hline \hline myc-thrombin-HA-MOG & $\begin{array}{l}\text { myc- and HA tags, separated by thrombin protease cleavage site, } \\
\text { fused to the extracellular N-terminus of MOG }\end{array}$ \\
pHluorin-MAG & pHluorin fused to the extracellular N-terminus of MAG \\
pHluorin-MOG & pHluorin fused to the extracellular N-terminus of MOG \\
pHluorin-PLPTM4 & pHluorin fused to the $4^{\text {th }}$ transmembrane domain of PLP \\
pHluorin-thrombin-MOG & pHluorin, thrombin protease cleavage site, MOG \\
SFV-VSVG-YFP & VSVG fused with YFP in alphaviral vector \\
\hline
\end{tabular}




\subsubsection{General Buffers and Solutions}

Phosphate Buffered Saline (PBS)

10X PBS was prepared according to [246]:

10X PBS ( 1 Ł)

80.0 g $\mathrm{NaCl}$

$2.0 \mathrm{~g} \mathrm{KCl}$

$14.4 \mathrm{~g} \quad \mathrm{Na}_{2} \mathrm{HPO}_{4}\left(\right.$ or $\left.18.05 \mathrm{~g} \mathrm{Na}_{2} \mathrm{HPO}_{4} \times 2 \mathrm{H}_{2} \mathrm{O}\right)$

$2.4 \mathrm{~g} \quad \mathrm{KH}_{2} \mathrm{PO}_{4}$

To obtain $1 \mathrm{X}$ PBS, 10X PBS was diluted 10 times with $\mathrm{ddH}_{2} \mathrm{O}$, $\mathrm{pH}$ value was adjusted to 7.4 .

\section{Protease Inhibitors (PI)}

Efficient inhibition of a wide spectrum of proteases was necessary in a variety of assays performed in this study [247]. For this, we used protease inhibitors cocktail Complete Mini (Roche Applied Science, Mannheim, Germany). PI stock solution: 1 tablet/1 mL PBS, aliquots stored at $-20^{\circ} \mathrm{C}$. To supply PBS, lysis buffer or other solution with protease inhibitors, PI stock was added to the solutions in a dilution 1:20 - 1:50 directly before use.

\section{Inhibitors for Cell Culture Assays}

All inhibitors used for various cell culture treatments (see Table 2.4) were purchased from Sigma-Aldrich (Sigma-Aldrich Chemie GmbH, Munich, Germany) or AppliChem (AppliChem GmbH, Darmstadt, Germany). Stock solutions were prepared under sterile conditions.

\subsubsection{Commercial Kits}

Commercial kits used in this project are listed in Table 2.5. 
Table 2.4: Inhibitors

\begin{tabular}{lllll}
\hline Name & Stock concentration & Dilution & Application & Storage \\
\hline \hline Brefeldin A & $10 \mathrm{mg} / \mathrm{mL}$ in ethanol & $1: 1000$ & $10 \mu \mathrm{g} / \mathrm{mL}, 15 \mathrm{~min}-2 \mathrm{~d}$ & $-20^{\circ} \mathrm{C}$ \\
Cycloheximide & $100 \mathrm{mg} / \mathrm{mL}$ in DMSO & $1: 2000$ & $50 \mu \mathrm{g} / \mathrm{mL}, 2-6 \mathrm{~h}$ & $+4^{\circ} \mathrm{C}$ \\
Cytocholasin D & $4 \mathrm{mM}$ in DMSO & $1: 2000$ & $2 \mu \mathrm{M}, 1-3 \mathrm{~h}$ & $-20^{\circ} \mathrm{C}$ \\
Fumonisin B1 & $1 \mathrm{mM}(1 \mathrm{mg}+1.38 \mathrm{~mL} \mathrm{PBS})$ & $1: 20$ & $50 \mu \mathrm{M}$, every $48 \mathrm{~h}, 4-7 \mathrm{~d}$ & $+4^{\circ} \mathrm{C}$ \\
Latrunculin A & $1 \mathrm{mM} \mathrm{in} \mathrm{H}_{2} \mathrm{O}$ & $1: 200$ & $5 \mu \mathrm{M}, 1-3 \mathrm{~h}$ & $-20^{\circ} \mathrm{C}$ \\
Nocodazole & $5 \mathrm{mg} / \mathrm{mL}$ in DMSO & $1: 2000$ & $2.5 \mu \mathrm{g} / \mathrm{mL}, 1-3 \mathrm{~h}$ & $-20^{\circ} \mathrm{C}$ \\
\hline
\end{tabular}

Table 2.5: Commercial kits

\begin{tabular}{lll}
\hline Commercial kit & Application & Manufacturer \\
\hline \hline Basic Neuron Nucleofector Kit & SiRNA transfection & Lonza, Basel, Switzerland \\
NucleoBond Xtra Midi kit & DNA isolation, medium scale & Macherey-Nagel, Dueren, Germany \\
NucleoSpin Extract II kit & DNA extraction from agarose gels & Macherey-Nagel, Dueren, Germany \\
NucleoSpin Plasmid kit & DNA isolation, small scale & Macherey-Nagel, Dueren, Germany \\
RiboMAX RNA Production, SP6 & in vitro transcription & Promega Corp., Madison, WI, USA \\
SuperScript III First-Str. Synth. & cDNA synthesis & Invitrogen, Carlsbad, CA, USA \\
QIAprep Spin Miniprep kit & DNA isolation, small scale & Qiagen, Hilden, Germany \\
QIAquick Gel Extraction kit & DNA extraction from agarose gels & Qiagen, Hilden, Germany \\
\hline
\end{tabular}

\subsubsection{Specific Software}

List of specific software used for assay design, data acquisition, processing and analysis can be found in Table 2.6. The software/applications marked with $\dagger$ are freely available online.

\subsection{Methods}

\subsubsection{Molecular Biology}

Molecular biological experiments were carried out in safety level S1 genetic laboratory according to $\mathrm{S} 1$ safety rules. 
Table 2.6: List of software

\begin{tabular}{lll}
\hline Software & Application & Source/Manufacturer \\
\hline \hline ApE $\dagger$ & DNA editing & by Wayne Davis, University of Utah \\
ImageJ $\dagger$ & Image processing and analysis & http://rsbweb.nih.gov/ij/ \\
Leica Confocal Software, 2.61 & Acquisition of confocal images & Leica Microsystems, Mannheim, Germany \\
LightCycler 480, 1.5.0. SP 3 & Acquisition of real-time PCR data & Roche Diagn. GmbH, Mannheim, Germany \\
MBF ImageJ for Microscopy $\dagger$ & Image processing and analysis & http://wwwacbiophotonics.ca/imagej/ \\
Meta Imaging Series 6.1 & Image processing and analysis & Universal Imaging Corp., Downingtown, USA \\
SigmaPlot 11 & Statistical analysis, curve fitting & Systat Software GmbH, Erkrath, Germany \\
Universal ProbeLibrary $\dagger$ & Primers design for real-time PCR & http://www.roche-applied-science.com \\
\hline
\end{tabular}

\section{Extraction and Purification of Plasmid DNA}

Plasmid DNA was amplified in DH5 alpha E. coli strains (Subcloning Efficiency DH5 alpha, Invitrogen, Carlsbad, CA, USA).

For large-scale purification, bacteria were inoculated in $100 \mathrm{~mL} \mathrm{LB}$ medium ${ }^{1}$ (LB-Medium $25 \mathrm{~g} / 1 \mathrm{ŁH}_{2} \mathrm{O}$, autoclaved) with appropriate antibiotics $(100 \mu \mathrm{g} / \mathrm{mL}$ Ampicillin or $50 \mu \mathrm{g} / \mathrm{mL}$ Kanamycin according to [246] and incubated at $37^{\circ} \mathrm{C}$ for $16 \mathrm{~h}$ with constant shaking. The cells were harvested by centrifugation at $4000 \mathrm{~g}$ for 20 min at $4^{\circ} \mathrm{C}$ and the plasmids were isolated and purified with NucleoBond Xtra Midi kit (Macherey-Nagel, Dueren, Germany) according to manufacturerer's instructions. The bacterial lysis in this procedure is performed based on the $\mathrm{NaOH} / \mathrm{SDS}$ lysis method by H. C. Birnboim and J. Doly [249]. Plasmid DNA is bound to the NuleoBond Xtra Silica Resin, which is a patented anion-exchange resin. Negative phosphate oxygen of the DNA backbone interacts ionically with positively charged methyl-hydroxyethyl-amino group on the resin. Elution of DNA is carried out in high-salt conditions by shift of $\mathrm{pH}$ from 6.5 to 9.0. Eluted DNA was precipitated with isopropanol, washed with $70 \%$ ethanol and redissolved in sterile $\mathrm{H}_{2} \mathrm{O}$ or in Tris/HCl pH 8.0. Plasmid yield was determined by UV-spectrophotometry; the DNA concentration was adjusted to $1 \mu \mathrm{g} / \mu \mathrm{L}$.

\footnotetext{
${ }^{1} \mathrm{LB}$ medium - Lysogeny broth, also known as Luria-Bertani medium, based on formulations of G. Bertani [248] is a classic enriched bacterial growth medium that is used for preparation of plasmid DNA and recombinant proteins, especially in E. coli.
} 
For colonies check after cloning, plasmids were amplified in $3 \mathrm{~mL}$ bacteria cultures. Bacterial colonies were inoculated in LB medium with appropriate antibiotics selection (see above) and grown at $37^{\circ} \mathrm{C}$ for $16 \mathrm{~h}$ with vigorous shaking. From these cultures, the plasmids were purified with either NucleoSpin Plasmid kit (MachereyNagel, Dueren, Germany) or QIAprep Spin Miniprep kit (Qiagen, Hilden, Germany). The procedure is based on the method by H. C. Birnboim and J. Doly [249] and was carried out according to manufacturer's instructions.

\section{Determination of DNA and RNA Concentration}

Nucleic acid concentration was determined from its optical density at $260 \mathrm{~nm}$ (A260) and calculated using the Beer-Lambert law [250]. An optical density of 1.0 at $260 \mathrm{~nm}$ measured in a cuvette of path length $1.0 \mathrm{~cm}$, corresponds to a concentration of $50 \mu \mathrm{g} / \mathrm{mL}$ and $40 \mu \mathrm{g} / \mathrm{mL}$ for double-stranded DNA and for RNA, respectively. To determine double-stranded DNA or RNA concentration, the samples were diluted 100-fold and the absorbance was read at $260 \mathrm{~nm}$ in UVette cuvettes (Eppendorf AG, Hamburg, Germany) with BioPhotometer plus (Eppendorf AG, Hamburg, Germany). The concentration was calculated with the following formulae:

DNA concentration $(\mu g / m L)=(A 260) \times($ dilution factor $) \times(50 \mu g / m L)$,

RNA concentration $(\mu g / m L)=(A 260) \times($ dilution factor $) \times(40 \mu g / m L)$.

DNA and RNA purity was estimated from A260/A280 and A260/A230 ratios. The nitrogenous bases in nucleotides have an absorption maximum at about $260 \mathrm{~nm}$. In contrast to nucleic acids, proteins have a UV absorption maximum of $280 \mathrm{~nm}$, due mostly to the aromatic residues. Therefore an A260/A280 ratio below 1.8 indicates protein contamination of the sample. Ratios of A260/A280 about 1.8 and 2.0 indicate pure plasmid DNA and pure RNA, correspondingly. This can be explained from the fact, that five bases that comprise DNA and RNA exhibit widely varying A260/A280 ratios: Guanine 1.15, Adenine 4.50, Cytosine 1.51, Uracil 4.00, Thymine 1.47 [250]. Typically, RNA has higher A260/A280 ratio due to the higher ratio of Uracil compared to that of Thymine. Absorption at $230 \mathrm{~nm}$ reflects 
contamination of the sample by phenols or aromatic compounds (e.g.. TRIzol). In the case of pure samples, the ratio A260/A230 is approximately $2.0-2.2$.

\section{DNA Analysis with Agarose Gels}

Electrophoresis through agarose gel was used to separate, analyze and purify DNA fragments. This method is based on the principle that in an electric field molecules of double-stranded DNA migrate through gel matrices at rates that are inversely proportional to the $\lg \left(\log _{10}\right)$ of the numbers of base pairs [251]. Electrophoresis was performed in custom-made chambers (Feinmechanik of the Max Planck Institute of Experimental Medicine) in TAE electrophoresis buffer (see composition below). 1\% or $2 \%$ agarose gels were prepared in TAE buffer and run at $5 \mathrm{~V} / \mathrm{cm}$. Gel-loading buffer (6X Loading dye) and marker DNA of known size (1 kb DNA ladder) were purchased from Fermentas (St. Leon-Rot, Germany). For detection of DNA in agarose gels a fluorescent intercalating dye ethidium bromide (EtBr) was added into gel solutions to a final concentration $0.5 \mathrm{mg} / \mathrm{mL}$ [252]. Detection was carried by direct examination of the gels in UV light with Intas Gel Documentation System (Intas Science Imaging Instruments GmbH, Göttingen, Germany).

\section{X TAE (1 L)}

$48.4 \mathrm{~g}$ Tris base

$11.4 \mathrm{~mL}$ Acetic acid

$20.0 \mathrm{~mL} \quad 0.5 \mathrm{M}$ EDTA

The volume was adjusted to $1 \mathrm{~L}$ with $\mathrm{ddH}_{2} \mathrm{O}$,

$\mathrm{pH}$ was adjusted to 8.5 .

\section{DNA Extraction from Agarose Gels}

After separation on agarose gels, DNA fragments were purified with NucleoSpin Extract II kit (Macherey-Nagel, Dueren, Germany) or QIAquick Gel Extraction kit (Qiagen, Hilden, Germany).

The gel extraction procedure involves dissolving the DNA-containing agarose in chaotropic salt solution (guanidine thiocyanate for both kits) and is based on the method described by B. Vogelstein and D. Gillespie [253]. The preparative 
technique uses binding of DNA to glass (special silica membrane in spin-columns for both kits) in the presence of chaotropic salt. Contaminations are removed by ethanolic washing step. To achieve the highest concentration of the DNA fragments, DNA is eluted in minimal volume $(15 \mu \mathrm{L}$ for NucleoSpin Extract II kit, $30 \mu \mathrm{L}$ for QIAquick Gel Extraction kit) of slightly alkaline low ionic strength elution buffer (5 - $10 \mathrm{mM}$ Tris/HCl, $\mathrm{pH} 8$ - 8.5).

\section{Restriction Enzyme Digestion}

The discovery of restriction enzymes and their applications for molecular biology was made by W. Arber, D. Nathans and H. Smith, for which they were awarded the Nobel Prize in 1978 [254-256]. We performed restriction enzyme digestions with the enzymes and buffer systems from New England Biolabs (Frankfurt am Main, Germany) and Fermentas (St. Leon-Rot, Germany). For each restriction reaction 5 - 10 units (U) of the enzymes were used, where one unit is defined as the amount of the enzyme required to digest $1 \mu \mathrm{g}$ of lambda DNA in $1 \mathrm{~h}$ at recommended temperature in $50 \mu \mathrm{L}$ of appropriate reaction buffer. The composition of the restriction reaction mixtures was as follows:

\section{Restriction mixture}

$1-2 \mu \mathrm{g} \quad$ DNA

$5 \mu \mathrm{L}$ appropriate 10X restriction buffer

$1 \mu \mathrm{L}$ each restriction enzyme $(5-10 \mathrm{U})$

Total reaction volume was adjusted with $\mathrm{H}_{2} \mathrm{O}$ to $50 \mu \mathrm{L}$.

The reaction mixtures were incubated for $1-4 \mathrm{~h}$ at appropriate temperature indicated by the enzyme provider.

\section{DNA Ligation}

DNA ligation was performed with bacteriophage T4 DNA ligase purchased from Fermentas (St. Leon-Rot, Germany). T4 DNA ligase catalyzes the formation of phosphodiester bonds between adjacent 3'-hydroxyl and 5'-phosphate termini in DNA [257]. The general set-up of a $10 \mu \mathrm{L}$ ligation reaction is described below: 


\section{Ligation mixture}

$1 \mu \mathrm{L}$ 10x T4 DNA ligase buffer (Fermentas, St. Leon-Rot, Germany)

$1 \mu \mathrm{L} \quad$ T4 DNA Ligase (1 - 5 Weiss U)

$1 \mu \mathrm{L} \quad$ vector DNA, restricted and purified, 50 - $100 \mathrm{ng}$

$7 \mu \mathrm{L}$ insert DNA, restricted and purified

(1:1 to 5:1 molar ratio insert:vector)

Reaction mixtures were incubated at RT for 30 - 60 min followed by incubation over night at $4^{\circ} \mathrm{C}$ for difficult to ligate fragments. $5 \mu \mathrm{L}$ of the ligation mixtures were directly used for transformation.

\section{Polymerase Chain Reaction (PCR)}

The polymerase chain reaction is a technique for specific enzymatic amplification of DNA segments in vitro. For the invention of PCR, K. Mullis [258] was awarded the Nobel Prize in 1993. In the original PCR protocols, which date back to the middle of 1980 s, the extension of annealed primers was catalyzed by the Klenow fragment of E. coli DNA polymerase I. The modern PCR technique uses thermostable DNA polymerase from Thermus aquaticus (Taq DNA polymerase) [259].

We routinely performed polymerase chain reactions with GoTaq $\AA$ DNA polymerase (Promega, Mannheim, Germany) in 5X Green GoTaq® flexi buffer to enable direct agarose gel analysis of amplified products. For preparing PCR reaction mixtures we followed the manufacturer's guidelines:

\section{PCR reaction mixture, general}

10 - $250 \mathrm{ng} \quad$ template DNA (approx. $10 \mathrm{ng}$ for plasmid DNA, up to $250 \mathrm{ng}$ for genomic DNA)

$1 \mathrm{X} \quad$ Green GoTaq $\AA$ flexi buffer

1.0 - $4.0 \mathrm{mM} \quad \mathrm{MgCl}_{2}$ solution

$0.2 \mathrm{mM} \quad$ each dNTP

$0.2-1.0 \mu \mathrm{M} \quad$ upstream primer

$0.2-1.0 \mu \mathrm{M} \quad$ downstream primer

$1.25 \mathrm{U} \quad$ GoTaq $\AA$ DNA Polymerase $(5 \mathrm{U} / \mu \mathrm{L})$

Reaction volume was adjusted to $20-50 \mu \mathrm{L}$ with nuclease-free water. 
We carried out PCR reactions in Multiply-Pro $0.5 \mathrm{~mL}$ Biosphere tubes (Sarstedt, Nuembrecht, Germany) in a T3000 Thermocycler Kombi (Biometra, Göttingen, Germany). Cycling profile for standard PCRs was as follows:

\title{
PCR amplification conditions, general
}

\author{
$95^{\circ} \mathrm{C} \quad 2-3 \mathrm{~min} \quad$ Initial denaturation
}

$95^{\circ} \mathrm{C} \quad 15-30 \mathrm{~s} \quad$ Denaturation

$55-68^{\circ} \mathrm{C} \quad 30 \mathrm{~s} \quad$ Annealing (temperature was adjusted according to the melting temperature of the primers)

$72^{\circ} \mathrm{C} \quad 15 \mathrm{~s}-3 \mathrm{~min} \quad$ Elongation (time was adjusted according to the length of the amplicon, $1 \mathrm{~kb} / \mathrm{s}$ )

Steps 2 - 4 were repeated 25 - 35 times

$72^{\circ} \mathrm{C} \quad 10 \mathrm{~min} \quad$ Final elongation

After the PCRs were performed, the reaction mixtures were loaded directly onto $1 \%$ or $2 \%$ agarose gels for analysis or stored over night at $4^{\circ} \mathrm{C}$ before analysis.

Specific reaction conditions for shiverer genotyping PCRs are described in Section 2.2.1.

For amplification of the DNA sequences for cloning purposes we used Phusion DNA polymerase (2X Phusion@ High-Fidelity PCR Master Mix with HF Buffer, Finnzymes, Espoo, Finland). This polymerase was chosen for its proofreading activity, high fidelity, speed and efficiency. Phusion@ DNA polymerase is a Pyrococcus-like enzyme with a processivity enhancing domain. It possesses $5^{\prime} \rightarrow 3^{\prime} \mathrm{DNA}$ polymerase and $3 \rightarrow 5^{\prime}$ exonuclease activities, and has an error rate 50 -fold lower than Taq DNA Polymerase.

For setting up PCRs with Phusion@ DNA polymerase, the following components were combined in PCR tubes on ice: Phusion $2 \mathrm{X}$ master mix $(25 \mu \mathrm{L}), 10 \mathrm{ng}$ of plasmid DNA template (1 $\mu \mathrm{L}$ of 1:100 dilution of midi-preps), $0.5 \mu \mathrm{M}$ forward primer, $0.5 \mu \mathrm{M}$ reverse primer $(0.5 \mu \mathrm{L}$ of $50 \mathrm{pmol} / \mu \mathrm{L}$ primer stock $)$ and the total reaction volume was adjusted to $50 \mu \mathrm{L}$ with $\operatorname{ddH}_{2} \mathrm{O}(23 \mu \mathrm{L})$.

Cycling conditions for PCRs with Phusion@ DNA polymerase were adjusted according to the manufacturer's instructions. In contrast to other polymerases, Phusion $囚$ DNA polymerase requires higher temperature for the denaturation steps 
$\left(98^{\circ} \mathrm{C}\right)$, annealing at $3^{\circ} \mathrm{C}$ above the primers' melting temperature and only 15 $30 \mathrm{~s} / \mathrm{kb}$ for extension.

\section{Primer Design}

PCR primer design was carried out with ApE free software for DNA engineering (ApE - A Plasmid Editor ${ }^{2}$ by M. Wayne Davis, University of Utah). PCR primers were designed to flank the region of interest on the template DNA. The length of the complementary to the target DNA primer region was chosen between 20 and 30 bases, so that both forward and reverse primers would have equal melting temperatures. The melting temperatures calculations are based on the nearest neighbour method [260, 261]. Primers were selected to contain between 40 and $60 \%(\mathrm{G}+\mathrm{C})$ (sum of guanine and cytosine bases in the primer); the preferred terminal nucleotide at the 3' end of the designed primers was $\mathrm{G}$ or $\mathrm{C}$. When required, primers were further checked for self-dimerization and hairpin formation.

The following considerations were taken into account when designing primers for further cloning of the amplicons (generation of fusion proteins, fluorescent protein tagging). When necessary for cloning, extra sequences for new restriction sites were introduced at 5' ends of the primers, flanked from the 5' end by 2 - 3 additional adenine nucleotides to ensure efficient enzymatic cleavage of the corresponding PCR products [262]. Care was taken, that the ectopic sequences do not introduce a frame shift or stop codons (when not intended). For generation of fusion proteins, extra codons for glycine amino acid residues were introduced in primers to provide a linker between the fused peptides.

Desoxyoligonucleotide synthesis was performed by the ACGTLab, DNA core facility of the Max Planck Institute of Experimental Medicine, Göttingen, Germany, according to the phosphoramidite method on a 3900 DNA Synthesiser from Applied Biosystems (Foster City, CA, USA) [263].

\footnotetext{
${ }^{2}$ http://www.biology.utah.edu/jorgensen/wayned/ape/
} 


\section{Site-Directed Mutagenesis}

In order to introduce point substitutions or deletions into proteins at amino acid level, mutagenesis was performed with Stratagene QuickChange ${ }^{\mathrm{TM}}$ site-directed mutagenesis kit (Agilent Technologies, Waldbronn, Germany) according to manufacturers instructions. The method is based on the site-specific mutagenesis by overlap extension principle described in [264].

\section{Transformation of Competent E. coli}

Bacterial transformation methods are based on the observations made by M. Mandel and A. Higa [265] and later on by S. Cohen and colleagues [266], who showed that treatment of bacteria with ice-cold $\mathrm{CaCl}_{2}$ salt solutions followed by brief heating allows transfection of bacteria with bacteriophage lambda DNA and transformation with plasmid DNA.

For plasmid amplification purposes we used Subcloning Efficiency DH5alpha (Invitrogen, Carlsbad, CA, USA) competent cells. For cloning experiments, Library Efficiency DH5 alpha Competent Cells (Invitrogen, Carlsbad, CA, USA) were employed. The latter were chosen for their improved transformation efficiency, due to the specific preparation procedure, based on the studies of D. Hanahan [267].

For transformation, $50-100 \mu \mathrm{L}$ of chemicompetent E. coli cells were thawed on ice. 10 - $100 \mathrm{pg}$ of the desired plasmid DNA or $5 \mu \mathrm{L}$ of the ligation mixture were added into the cell suspension and mixed, followed by 30 min incubation on ice, $45 \mathrm{~s}$ at $42^{\circ} \mathrm{C}$ heat-shock and recovery on ice for $2 \mathrm{~min}$. After addition of 500 $1000 \mu \mathrm{L}$ LB medium, the cells were incubated for $1 \mathrm{~h}$ at $37^{\circ} \mathrm{C}$ with agitation. Cells were pelleted by brief centrifugation, resuspended in $100-200 \mu \mathrm{L}$ of LB, spread on LB plates (LB-Agar $40 \mathrm{~g}$ per $1 \mathrm{~L} \mathrm{H}_{2} 0$, autoclaved) with appropriate antibiotics selection [246] and incubated at $37^{\circ} \mathrm{C}$ for $16 \mathrm{~h}$.

\section{DNA Sequencing}

DNA sequencing reactions were performed by the ACGTLab, DNA core facility of the Max Planck Institute of Experimental Medicine, Göttingen, Germany, according to the Sanger method [268] using the Big Dye Terminator Kit v3.1 from Applied 
Biosystems and analyzed on a 3730XL DNA-Analyzer from Applied Biosystems (Foster City, CA, USA).

\section{Cloning of Viral Vectors, Production of Recombinant SFV}

To achieve efficient synchronized high-level expression of the VSVG-ts042-EYFP transgene in primary oligodendrocytes, we employed Semliki Forest virus expression system, developed by P. Liljeström and co-workers [243].

Semliki Forest virus (SFV) is a positive-strand RNA virus of the alphavirus genus. In a viral vector ("SFV vector"), a transgene of interest replaces the viral structural protein genes, which are required for encapsidation of viral RNA. To be packaged into infectious particles, the SFV vector must be co-transfected with a "Helper" vector, which provides the structural proteins for replicon assembly. We chose the SFV/Helper2 system [244] for additional safety, which it offers due to introduced mutations in the viral spike proteins, encoded by Helper2, rendering viral particles noninfectious after packaging. To infect cultured cells, these packaged replicons must be activated with high concentrations of $\alpha$-chymotrypsin.

The VSVG-ts042-EYFP transgene was subcloned from VSVG3-SP-YFP (see Table 2.2) into the multiple cloning region of vector plasmid pSFV2gen (Invitrogen, Carlsbad, CA, USA) using XhoI and NotI restriction sites.

After linearization of the vector and helper plasmids with NruI and SpeI restriction enzymes respectively, in vitro transcription of the pSFV2gen carrying VSVGts042-EYFP transgene and of the Helper plasmid pSFV-Helper2 was performed with RiboMAX Large Scale RNA Production System, SP6 (Promega Corp., Madison, WI, USA) system according to manufacturer's instructions.

Packaging and activation of SFV replicons was performed according to the original technique $[243,269]$ with slight modifications, as detailed in the protocol by M. Ehrengruber [270]. In brief, recombinant vector RNA was mixed with Helper2 RNA and electroporated in BHK-21 cells. After $24 \mathrm{~h}$, cell growth medium containing packaged replicons was harvested, aliquoted, snap-frozen in $\mathrm{N}_{2}$ (l) and stored at $70^{\circ} \mathrm{C}$ until activation. Activation of viral replicons was performed by incubating the virus-containing supernatant with $200 \mu \mathrm{g} / \mathrm{mL} \alpha$-chymotrypsin $(96 \mathrm{U} / \mathrm{mg}$, SigmaAldrich Chemie GmbH, Munich, Germany) for 30 min at RT and terminated by 
addition of $1 \mathrm{mg} / \mathrm{mL}$ aprotinin (Sigma-Aldrich Chemie GmbH, Munich, Germany) for $10 \mathrm{~min}$ at RT. Primary oligodendrocytes were infected by incubation with infectious replicons for $1 \mathrm{~h}$ at $37^{\circ} \mathrm{C}$ in $7.5 \% \mathrm{CO}_{2}$ incubator. The cells were fixed every hour starting with $4 \mathrm{~h}$ after infection to observe time-dependent incorporation of VSVG protein into oligodendroglial plasma membrane.

\section{Total RNA Isolation}

Total RNA extraction from the TRIzol@-lysed mammalian cells was performed according to the instructions for using TRIzol@ Reagent (Invitrogen, Carlsbad, CA, USA). This method is based on the protocol for single-step RNA extraction with acid guanidinium thiocyanate-phenol-chloroform mixture described by P. Chomczynski and N. Sacchi [271].

\section{Denaturing Agarose Gel Electrophoresis of RNA}

After extraction, the quality of the total RNA was checked by electrophoresis in formaldehyde containing agarose gels [246]. The compositions of the electrophoresis buffers (Running buffer 10X and 1X) and of the loading dye can be found below. All the solutions were prepared with diethylpyrocarbonate (DEPC) treated $\mathrm{H}_{2} \mathrm{O}$ $\left(\mathrm{DEPC}-\mathrm{H}_{2} \mathrm{O}\right)$.

To prepare DEPC- $\mathrm{H}_{2} \mathrm{O}, 1 \mathrm{~mL}$ DEPC was added to $1 \mathrm{E} \mathrm{H}_{2} \mathrm{O}$, incubated at $37^{\circ} \mathrm{C}$ for $24 \mathrm{~h}$ to inactivate RNases and subsequently autoclaved.

\section{X Running buffer}

$200 \mathrm{mM}$ 3-(N-Morpholino)propanesulfonic acid (MOPS)

$50 \mathrm{mM}$ Sodium acetate

$10 \mathrm{mM} \quad$ EDTA, $\mathrm{pH} 7.0$

Adjustment of the $\mathrm{pH}$ to 7.0 was done with $1 \mathrm{M} \mathrm{NaOH}$.

To prepare 1X Running buffer (1 L), $100 \mathrm{~mL}$ 10X Running buffer, $20 \mathrm{~mL} 37 \%$ Formaldehyde and $880 \mathrm{~mL}$ DEPC- $\mathrm{H}_{2} 0$ were combined.

To prepare $10 \mathrm{~mL}$ of $5 \mathrm{X}$ Loading dye, $100 \mu \mathrm{L}$ saturated bromophenol blue (in DEPC- $\mathrm{H}_{2} \mathrm{O}$ ), $80 \mu \mathrm{L} 0.5 \mathrm{M}$ EDTA (pH 8.0), $720 \mu \mathrm{L} \mathrm{37 \%}$ formaldehyde, $2.0 \mathrm{~mL}$ glyc- 
erol, $3.0 \mathrm{~mL}$ de-ionized formamide, and $4.0 \mathrm{~mL}$ Running buffer (10X) were combined. The volume was adjusted to $10 \mathrm{~mL}$ with $\mathrm{DEPC}-\mathrm{H}_{2} 0$. The solution was aliquoted and stored at $-20^{\circ} \mathrm{C}$.

To prepare a gel, $1.2 \mathrm{~g}$ Agarose and $10 \mathrm{~mL}$ Running buffer (10X) were combined, the volume was adjusted to $100 \mathrm{~mL}$ with $\mathrm{DEPC}-\mathrm{H}_{2} \mathrm{O}$. The mixture was heated to melt agarose, cooled to $65^{\circ} \mathrm{C}$ in a water bath, and combined with $1.8 \mathrm{~mL}$ of $37 \%$ formaldehyde and $2.0 \mu \mathrm{L}$ of $10 \mathrm{mg} / \mathrm{mL}$ EtBr stock. After polymerization, the gels were equilibrated with $1 \mathrm{X}$ Running buffer for at least $30 \mathrm{~min}$ before running the gel.

To prepare the samples, 1 volume of $5 \mathrm{X}$ Loading dye was added per 4 volumes of RNA sample (approx. $1 \mu \mathrm{g}$ RNA). The samples were incubated at $65^{\circ} \mathrm{C}$ for 3 5 min, chilled on ice and loaded onto the equilibrated agarose gel. The gels were run at $80 \mathrm{~V}(5-7 \mathrm{~V} / \mathrm{cm})$.

\section{cDNA Synthesis}

cDNA was synthesized by RT-PCR with the SuperScript@ III First-Strand Syn-

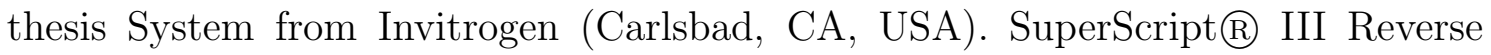
Transcriptase is a version of Moloney Murine Leukemia Virus Reverse Transcriptase (M-MLV RT) with reduced RNase H activity and increased thermal stability [272]. cDNA synthesis was performed from purified total RNA (approx. $1 \mu \mathrm{g}$ per reaction) primed with oligo(dT) primer according to manufacturer's instructions: For reverse transcription of total RNA, oligo(dT) primers and dNTPs were combined in the following amounts:

\section{RNA-mix, 1 sample}

$\mathrm{X} \mu \mathrm{L} \quad \mathrm{RNA}$ (approx. $1-5 \mu \mathrm{g}$ )

$1 \mu \mathrm{L} \quad 50 \mu \mathrm{M}$ oligo(dT) primer

$1 \mu \mathrm{L} \quad 10 \mathrm{mM}$ dNTP mix

The total volume was adjusted with sterile $\mathrm{H}_{2} \mathrm{O}$ to $10 \mu \mathrm{L}$.

The RNA-mix was incubated at $65^{\circ} \mathrm{C}$ for $5 \mathrm{~min}$, then cooled down to $4^{\circ} \mathrm{C}$. To each RNA-mix, $10 \mu \mathrm{L}$ of RT-mix was added, collected by brief centrifugation and incubated at $50^{\circ} \mathrm{C}$ for 50 min. RT-mix composition: 


\section{RT-mix, 1 sample}

$2 \mu \mathrm{L} \quad$ 10X RT buffer

$4 \mu \mathrm{L} \quad 25 \mathrm{mM} \mathrm{MgCl}_{2}$

$2 \mu \mathrm{L} \quad 0.1 \mathrm{M}$ DTT

$1 \mu \mathrm{L} \quad$ RNaseOUT $(40 \mathrm{U} / \mu \mathrm{L})$

$1 \mu \mathrm{L} \quad$ Superscript III RT $(200 \mathrm{U} / \mu \mathrm{L})$

The reaction was terminated by incubation for $5 \mathrm{~min}$ at $85^{\circ} \mathrm{C}$ and collected by brief centrifugation. To digest the RNA templates, each reaction mix was incubated with $1 \mu \mathrm{L}$ RNase $\mathrm{H}(2 \mathrm{U} / \mu \mathrm{L})$ at $37^{\circ} \mathrm{C}$ for $20 \mathrm{~min}$. After the reverse transcription was carried out, the reaction mixtures were diluted and used as a template for realtime or semi-quantitative PCR in order to estimate relative amounts of the specific transcripts in the original RNA extracts.

\section{Quantitative Real-Time PCR}

Quantitative real-time PCR is one of the key technologies for gene expression analysis $[273,274]$. We employed this technique for relative determination of copy numbers of the cDNA templates generated by RT-PCR from the total RNA extracts (see above) in order to analyze FB1 effects on murine primary oligodendrocytes.

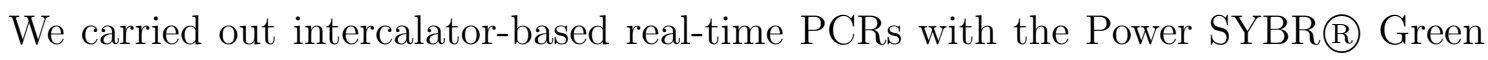
PCR Master Mix (Applied Biosystems, Warrington, UK). The SYBR@ Green dye binds to double-stranded DNA generated during polymerase chain reaction, thus providing a fluorescent signal that reflects the amount of the PCR product.

All primers for real-time PCR were designed to have melting temperatures $59-60^{\circ} \mathrm{C}$ and to generate intron-spanning amplicons (exception: rat and mouse MBPexVII primers) of uniform length (60 - 100 nt). Assay Design Software Universal ProbeLibrary ProbeFinder ${ }^{3}$ (Roche) was used for selecting the primers for this application. List of primers ${ }^{4}$ used for gene expression analysis can be found in Table 2.7.

\footnotetext{
${ }^{3} \mathrm{http}: / /$ www.roche-applied-science.com

${ }^{4}$ Indications "m", "r" and "u" in primer names stand for mouse (Mus musculus), rat (Rattus norvegicus) and universal primers (applicable for both species) respectively.
} 
Real-time polymerase chain reactions were performed in a LightCycler@ 480 equipped with a high-intensity xenon lamp and a charge-coupled device (CCD) camera, which monitors fluorescence in each well of a 96-well plate. LightCycler@ 480 Multiwell Plates 96 covered with LightCycler@ 480 Sealing Foils. All LightCycler products were supplied by Roche Diagnostics GmbH, Mannheim, Germany.

\section{Real-time PCR reaction mixture, 1 sample \\ $5.0 \mu \mathrm{L}$ Power SYBR@ Green PCR Master Mix \\ $0.1 \mu \mathrm{L} \quad$ gene-specific primer, left, stock $10 \mathrm{pmol} / \mu \mathrm{L}$ \\ $0.1 \mu \mathrm{L}$ gene-specific primer, right, stock $10 \mathrm{pmol} / \mu \mathrm{L}$ \\ $0.8 \mu \mathrm{L} \quad \mathrm{dd}_{2} \mathrm{O}$ \\ $4.0 \mu \mathrm{L} \quad \mathrm{cDNA}$ template (reaction mixture after reverse transcription diluted $1: 10)$}

The plates were centrifuged for $1 \mathrm{~min}$ at $1400 \mathrm{rpm}$ in an Eppendorf $5810 \mathrm{R}$ centrifuge (Eppendorf AG, Hamburg, Germany) before being placed in the LightCycler. The amplification conditions for the real-time PCR assays were as follows:

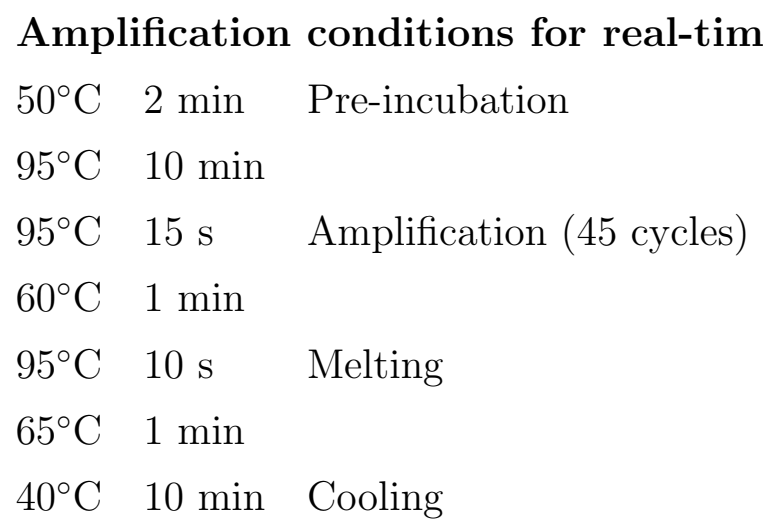

Relative changes in gene expression were quantified from real-time PCR experiments using the standard $\Delta \Delta \mathrm{C}_{T}$ method [275], where $\mathrm{C}_{T}$ is the threshold cycle, indicating the fractional cycle number at which the amount of amplified target reaches a fixed automatically set threshold. The absence of nonspecific amplification was confirmed by the dissociation curve analysis [274]. 
Table 2.7: Primers for real-time PCR

\begin{tabular}{lll}
\hline Number & Name & Sequence \\
\hline \hline 11282 & mATP5b, left & GGATCTGCTGGCCCCATAC \\
11283 & mATP5b, right & CTTTCCAACGCCAGCACCT \\
11284 & mCNP, left & TAACCCTCCCTTAGCCCCTG \\
11285 & mCNP, right & GTCCCTAGCATGTGGCAGCT \\
4345 & mMAG, left & TTCGAGGGCACGTGTGTCT \\
4344 & mMAG, right & CACAGCCGGTCTGAGCTCA \\
8679 & mMBP, left & GCCTGTCCCTCAGCAGATT \\
8678 & mMBP, right & GCCTCCGTAGCCAAATCC \\
19748 & mMBPexVII, left & ACGGACACCCTTCCAAGTT \\
19749 & mMBPexVII, right & GTGTGCCTCACCGTGAAAA \\
19750 & mMBPexIII-V, left & CGGACCCAAGATGAAAACC \\
19751 & mMBPexIII-V, right & GACAGGCCTCTCCCCTTC \\
19641 & mMOG left & CTTCTTCAGAGACCACTCTTACCA \\
19642 & mMOG right & GTTGACCCAATAGAAGGGATCTT \\
19649 & mOlig2, left & AGACCGAGCCAACACCAG \\
19650 & mOlig2, right & AAGCTCTCGAATGATCCTTCTTT \\
\hline 19657 & rATP5b, left & GGGTACAATGCAGGAAAGAATC \\
19658 & rATP5b, right & TCAGCTGGCACATAGATAGCC \\
19647 & rCNPase, left & AAATTCTGTGACTACGGGAAGG \\
19648 & rCNPase, right & CCGTAAGATCTCCTCACCACA \\
19645 & rMBPexVII, left & GGACTGCAGGAGTTCTCTGG \\
19646 & rMBPexVII, right & GCTGACCACCCTAAAGTGAGA \\
19659 & rMBP69, left & GGCACGCTTTCCAAAATCT \\
19660 & rMBP69, right & CCATGGGAGATCCAGAGC \\
19661 & rMBP29, left & GCCTGTCCCTCAGCAGATT \\
19662 & rMBP29, right & TCTGCCTCCGTAGCCAAAT \\
19643 & rMOG, left & CCTCCTGTCCCTGCTCCT \\
19644 & rMOG, right & GCCCTATCACTCTGAACTGTCC \\
19655 & rOlig2, left & GCGCGAAACTACATCCTGA \\
19656 & rOlig2, right & CGTAAATCTCGCTCACCAGTC \\
\hline 11292 & uPLP, left & GGCTAGGACATCCCGACAAGT \\
11293 & uPLP, right & GGCAAACACCAGGAGCCATA \\
\hline & &
\end{tabular}

\section{Shiverer Mouse Line Maintenance and Genotyping}

Shiverer mouse line was maintained by breeding heterozygote carriers of the shiverer allele and genotyping their progeny according to [276]. For genotyping, genomic DNA was extracted from tail biopsy samples with Invisorb spin tissue mini kit (Invitek, Berlin, Germany) according to the manufacturer's instructions. The volume of the elution buffer used for the final step of the DNA extraction protocol was $50 \mu \mathrm{L}$. 
The presence of wild-type or Shiverer alleles in genomic DNA samples was detected using two sets of specific primers. One set of primers $(01873,01874)$ amplifies a 169 bp fragment from intron sequences flanking exon VI of the MBP gene in wildtype mouse DNA. As Shiverer mice are lacking exons II - VII, these primers do not generate a PCR product from the Shiverer allele. The second set of primers (01879, 01880) amplifies a $380 \mathrm{bp}$ fragment unique to the shiverer allele. The amplicon for these primers spans the deletion region of the MBP gene, which is characteristic for the Shiverer DNA. The primer sequences can be found in Table 2.8.

Table 2.8: Primers for Shiverer genotyping

\begin{tabular}{lll}
\hline Number & Name & Sequence \\
\hline \hline 01873 & MBP wt, rev & AGCTCTGGTCTTTCTTGCAG \\
01874 & MBP wt, forw & CCCCGTGGTAGGAATATTACATAAC \\
01879 & Shiv, rev & CAGGGGATGGGGAGTCAGAAGTGAG \\
01880 & Shiv, forw & ATGTATGTGTGTGTGTGCTTATCTAGTGTA \\
\hline
\end{tabular}

For genotyping PCRs, GoTaq®) Polymerase with 5X Green Buffer (Promega Corp., Madison, WI, USA) was used. The amplification mixture of the following composition (per sample) was as assembled on ice:

\section{PCR reaction mixture for Shiverer genotyping}

$0.5 \mu \mathrm{L}$ genomic DNA in elution buffer

$0.1 \mu \mathrm{L}$ primer $01873(50 \mathrm{pmol} / \mu \mathrm{L})$

$0.1 \mu \mathrm{L}$ primer $01874(50 \mathrm{pmol} / \mu \mathrm{L})$

$0.1 \mu \mathrm{L}$ primer $01879(50 \mathrm{pmol} / \mu \mathrm{L})$

$0.1 \mu \mathrm{L}$ primer $01880(50 \mathrm{pmol} / \mu \mathrm{L})$

$0.2 \mu \mathrm{L} \quad \mathrm{dNTPs}$ mix (10 mM each dNTP)

$4.0 \mu \mathrm{L} \quad 5 \mathrm{X}$ Green GoTaq Flexi buffer

$0.2 \mu \mathrm{L}$ GoTaq Polymerase $(5 \mathrm{U} / \mu \mathrm{L})$

$1.0 \mu \mathrm{L} \quad \mathrm{MgCl}_{2}$ (25 mM stock)

$13.7 \mu \mathrm{L} \quad \mathrm{ddH}_{2} \mathrm{O}$

High-efficiency amplification in a thermocycler (Biometra, Goettingen, Germany) in $0.2 \mathrm{~mL}$ Thermo-Strip thin-wall PCR tubes or Thermo-Fast 96-well PCR plates 
(both products from Thermo Scientific, Epsom, United Kingdom) was achieved using the following conditions:

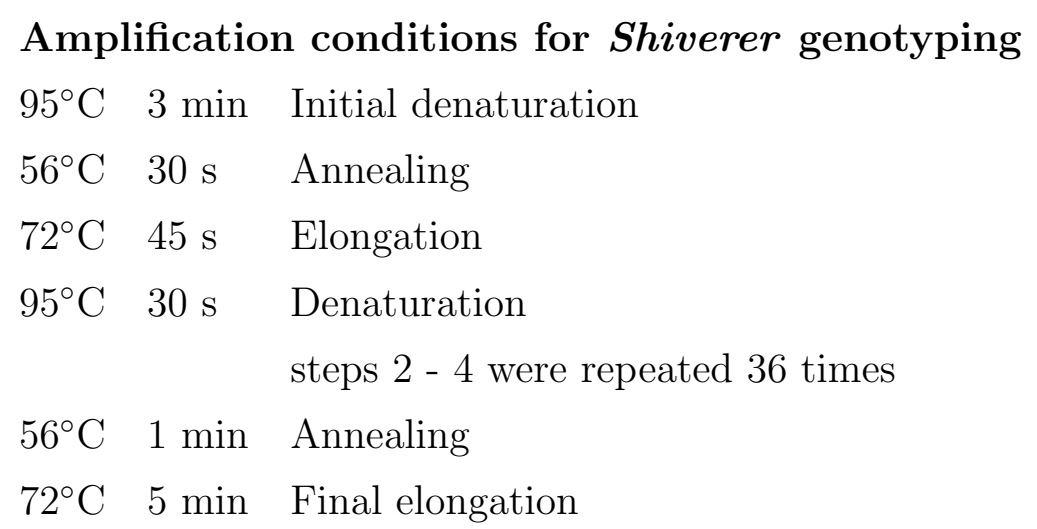

For visualization of amplified products, agarose gel analysis was performed in $2 \%$ agarose gels, as described in Section 2.2.1. Per lane, the whole reaction mixture was loaded.

Primary oligodendroglial cell cultures were prepared from mixed offspring from one litter obtained by crossing heterozygote Shiverer mice as described in Section 2.2.3. Identification of Shiverer oligodendrocytes in mixed primary cultures was done with immunostaining for $\mathrm{O} 1$ and MBP epitopes 6 days after shake. Only O1-positive and MBP-negative mature oligodendrocytes were regarded as Shiverer oligodendrocytes in these cultures.

\subsubsection{Biochemistry}

All chemicals were handled according to the safety requirements, provided by the manufacturer.

\section{Sodium Dodecyl Sulfate Polyacrylamide Gel Electrophoresis (SDS-PAGE)}

Analytical electrophoresis of proteins was carried out in polyacrylamide gels in presence of the ionic detergent sodium dodecyl sulfate (SDS) [277]. Before and during gel electrophoresis, SDS denatures proteins and adds negative charges to the polypeptide chains thus eliminating differences in shape so that molecular mass becomes the main determinant of the protein migration rate. Gel preparation was performed in Bio-Rad Mini-PROTEAN 3 casting system, separation of proteins according to 
their electrophoretic mobility was carried out with vertical electrophoresis systems Bio-Rad Mini-PROTEAN (Bio-Rad Laboratories GmbH, München, Germany) in Tris-glycine electrophoresis buffer (25 mM Tris, $192 \mathrm{mM}$ glycine, 0.1\% SDS) for $2 \mathrm{~h}$ at $100 \mathrm{~V}(15 \mathrm{~V} / \mathrm{cm})$. Resolving gel and stacking gels were of the following composition:

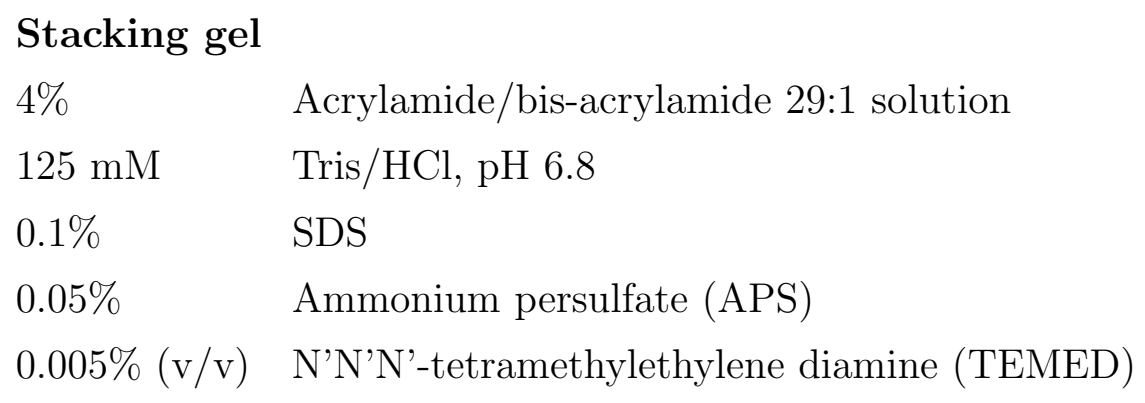

\section{Resolving gel (8 - 15\%)}

8 to $15 \% \quad$ Acrylamide/bis-acrylamide 29:1 solution

$375 \mathrm{mM} \quad$ Tris/HCl, $\mathrm{pH} 8.8$

$0.1 \% \quad$ SDS

$0.05 \% \quad$ APS

$0.005 \%(\mathrm{v} / \mathrm{v})$ TEMED

As a general rule, before loading on the gel, the samples were incubated at $95^{\circ} \mathrm{C}$ for $5 \mathrm{~min}$ in reducing sample buffer of the following composition:

\section{Sample buffer for SDS-PAGE}

$50 \mathrm{mM}$ Tris/ $\mathrm{HCl} \mathrm{pH} 6.8$

$10 \% \quad$ Glycerol

$2 \mathrm{mM} \quad$ EDTA

$2 \% \quad$ SDS

0.05\% Bromophenol blue

$144 \mathrm{mM} \quad \beta$-mercaptoethanol (approx. 2\%v/v)

For MOG and MAG analysis, non-reducing SDS-PAGE conditions were applied (incubation at $60^{\circ} \mathrm{C}$ for $10 \mathrm{~min}$ in sample buffer without $\beta$-mercaptoethanol). For PLP detection, incubation in sample buffer was performed at $55^{\circ} \mathrm{C}$ for $10 \mathrm{~min}$. Molecular weights of the analyzed proteins were estimated according to protein 
molecular weight marker PageRuler@ Plus Prestained Protein Ladder (Fermentas, St. Leon-Rot, Germany).

\section{Western Blotting}

For identification and quantification of electrophoretically separated proteins, Western blotting technique was employed [278]. After separation on SDS-polyacrylamide gels, the proteins were transferred onto a Whatman $\AA$ Protran Nitrocellulose Transfer Membrane (Whatman GmbH, Dassel, Germany). Protein transfer was carried out for $1 \mathrm{~h}$ at $100 \mathrm{~V}$ in a transfer buffer (25 mM Tris, $192 \mathrm{mM}$ glycine, 20\% methanol) in Bio-Rad Mini-Protein System (Bio-Rad Laboratories GmbH, München, Germany) according to manufacturer's instructions.

Further immunological detection was performed. To reduce nonspecific binding of immunoglobulins, the membranes with transferred proteins were incubated in $4 \%$ nonfat dried milk (Sigma-Aldrich Chemie GmbH, Munich, Germany) in PBS for 30 min at RT. Incubation with primary antibodies in appropriate dilution was carried out in PBST (PBS prepared as described in Section 2.1.4 and supplemented with $0.1 \%(\mathrm{v} / \mathrm{v})$ Tween-20) for $1 \mathrm{~h}$ at RT or for $16 \mathrm{~h}$ at $4^{\circ} \mathrm{C}$, followed by three $5 \mathrm{~min}$ washings with PBST. Incubation with secondary anti-mouse or anti-rabbit antibodies (purchased from Dianova, Hamburg, Germany) conjugated with horseradish peroxidase (HRP) in 1:2000 and 1:5000 dilution respectively was done for $1 \mathrm{~h}$ at RT, followed by three 5 min washings with PBST. Antigens were visualized by chemiluminescence with Pierce ECL Western Blotting Substrate from Thermo Scientific (Epsom, United Kingdom). HRP enzyme conjugated to the secondary antibodies oxidizes luminol substrate into an excited state product which emits light. Developed X-ray films (CL-XPosure ${ }^{\mathrm{TM}}$ Film, ThermoScientific, Rockford, IL, USA) with detected chemiluminescent signals were scanned with a conventional scanner and the signal densities were quantified with Image $\mathrm{J}^{5}$.

\footnotetext{
${ }^{5}$ http://rsbweb.nih.gov/ij/
} 


\section{Preparation of Cells for Biochemical Analysis}

For biochemical analysis, primary oligodendrocytes or Oli-neu cells were prepared as a native suspension of membranes and cytosolic components, as a postnuclear supernatant (SN) from cells treated with lysis buffer or as a lysate of cells in TRIzol reagent (cells harvested in TRIzol were further subjected to RNA, DNA and protein extraction).

Native suspension. Primary oligodendrocytes or Oli-neu cells were cultured in $6 \mathrm{~cm}$ petri dishes (see Section 2.2.3 and Section 2.2.3), washed once in PBS and detached from the plates by using a cell scraper (Sarstedt, Nümbrecht, Germany) into $150 \mu \mathrm{L}$ of ice-cold PBS supplemented with protease inhibitors. Cells were sheared on ice by passing 15 - 20 times through a $27 \mathrm{G}$ needle and cleared by centrifugation for $5 \mathrm{~min}$ at $300 \mathrm{~g}$ at $4^{\circ} \mathrm{C}$. These preparations were further used for fractionation according to heavy/light myelin preparation protocol, for detergent resistance tests, "semi-native" SDS-PAGE, etc.

Preparation of cell lysates. Primary oligodendrocytes or Oli-neu cells were cultured in $6 \mathrm{~cm}$ petri dishes (see Section 2.2.3 and Section 2.2.3), washed once in PBS and scraped on ice into $150 \mu \mathrm{L}$ of lysis buffer (2\% NP-40, 0.2\% SDS, $0.5 \mathrm{mM}$ EDTA in PBS) supplemented with protease inhibitors (see Section 2.1.4) [279]. Postnuclear supernatants were acquired by centrifugation of lysates for 5 min at $300 \mathrm{~g}$ at $4^{\circ} \mathrm{C}$. These preparations were further analyzed with SDS-PAGE and Western blotting.

TRIzol-based RNA, DNA and protein extraction. TRIzol@ reagent Invitrogen (Carlsbad, CA, USA) is a commercially available mono-phasic solution of phenol and guanidine isothiocyanate. For cell harvesting, $0.5 \mathrm{~mL}$ or $1 \mathrm{~mL}$ of TRIzol was applied directly to a 3.5 or $6 \mathrm{~cm}$ diameter culture dish after the growth medium was aspirated and the cells were washed once with sterile PBS. RNA extraction from TRIzol-lysed samples was performed according to manufacturers instructions, based on the method of RNA isolation by a single extraction with an acid guanidinium thiocyanate-phenol-chloroform mixture [271]. In brief, after addition of chlorophorm to the samples containing cells in TRIzol@ reagent, centrifugations were performed to separate the solutions into aqueous and organic phases. RNA was harvested from 
the aqueous phases, genomic DNA was precipitated from the phenol phase and interphase, followed by protein precipitation [280]. The resulting RNA preparations were used for cDNA synthesis in conjunction with real-time or semi-quantitative PCR to compare the amounts of specific transcripts in treated and control samples. The protein preparations were analyzed by Western blotting.

\section{Preparation of Total Membrane Fractions}

For the preparation of total membrane fractions [279], Oli-neu cells were cultured on $15 \mathrm{~cm}$ dishes (approx. 20 dishes were used for one membrane preparation) under normal growth conditions (as described in Section 2.2.3). After the cells have reached $80 \%$ confluence, their growth media was aspirated, the cells were washed once with PBS and scraped in isotonic buffer $(20 \mathrm{mM}$ Tris/HCl, $\mathrm{pH} 7.4,1 \mathrm{mM}$ $\mathrm{MgCl}_{2}$, supplemented with protease inhibitors (PI), see Section 2.1.4). The cells were homogenized by sonication; postnuclear supernatant was prepared by centrifugation at $1000 \mathrm{~g}$ for $5 \mathrm{~min}$ at $4^{\circ} \mathrm{C}$ in a Eppendorf $5810 \mathrm{R}$ centrifuge (Eppendorf AG, Hamburg, Germany). To pellet the membranes, the postnuclear supernatants were subjected to centrifugation at $100000 \mathrm{~g}$ for $30 \mathrm{~min}$ at $4^{\circ} \mathrm{C}$ in $(36800 \mathrm{rpm}$ in an SW70 Ti rotor) in a Beckmann XL-70 Ultracentrifuge (Beckman Coulter GmbH, Krefeld, Germany). The pellets were resuspended in $10 \mathrm{mM}$ Tris/HCl, pH 7.4, supplemented with $5 \mathrm{mM}$ EDTA and protease inhibitors (see Section 2.1.4) by passing through a 27 G needle.

\section{Preparation of Myelin Fractions}

For biochemical analysis, we used crude CNS myelin fractions (first part of the protocol), highly purified CNS myelin fractions (described in the second part of the protocol), or subfractions of different densities prepared from crude myelin fractions (outlined in the third part of the protocol). Myelin isolation methods rely on the large size and low density of myelin vesicles [43, 281]. These properties make differential and density gradient centrifugations the main tools for the isolation of this membrane. 
1. This protocol for isolation of myelin is based on the W. Norton and S. Poduslo method [282], with modifications introduced in [283]. Brains from P21 - P60 wild-type (WT) or knock-out (Shiverer and CerS2 KO) mice were homogenized in ice-cold PBS with protease inhibitors (see Section 2.1.4) by sonication, $1 \mathrm{~mL}$ PBS-PI per brain. Homogenates were resuspended in $0.32 \mathrm{M}$ sucrose and layered over $0.85 \mathrm{M}$ sucrose $(1$ brain per 2 Beckman $14 \times 89 \mathrm{~mm}$ ultraclear centrifuge tubes (Beckman Coulter GmbH, Krefeld, Germany). In these preparations, all sucrose solutions were supplemented with $5 \mathrm{mM}$ EDTA and $10 \mathrm{mM}$ HEPES, pH 7.4. After centrifugation at $75000 \mathrm{~g}$ for $30 \mathrm{~min}$ (20900 rpm in an Sw41-Ti rotor in a Beckman XL-70 Ultracentrifuge (Beckman Coulter GmbH, Krefeld, Germany) the interfaces between 0.32 M and 0.85 M sucrose were collected, diluted at least 10 times with ice-cold $\mathrm{H}_{2} \mathrm{O}$ and centrifuged again at $75000 \mathrm{~g}$ for $30 \mathrm{~min}$ to pellet the fractions. The pellets were washed twice with ice-cold $\mathrm{H}_{2} \mathrm{O}$ (osmotic shock) and recovered by 10 min centrifugation at $12000 \mathrm{~g}$ (8500 rpm in an Sw41-Ti rotor). Low-speed centrifugations were performed to remove cytoplasmic and microsomal contaminants. This preparation yields crude myelin fractions, which can be further used for obtaining purified myelin or for density fractionation into heavy, medium and light fractions.

2. Purified myelin can be obtained by subjecting the crude myelin fraction to a repetition of the protocol described above. The pellets of purified myelin fractions were resuspended in $\mathrm{H}_{2} \mathrm{O}$ or in PBS-PI (see Section 2.1.4).

3. Crude myelin can be subfractionated further by discontinuous density gradient centrifugation according to [284, 285]. For this, suspension of crude myelin was layered over a four-step sucrose gradient $(0.32 \mathrm{M}, 0.62 \mathrm{M}, 0.7 \mathrm{M}$ and $1 \mathrm{M}$ sucrose in $5 \mathrm{mM}$ EDTA and $10 \mathrm{mM}$ HEPES, pH 7.4) and centrifuged at $75000 \mathrm{~g}$ for $30 \mathrm{~min}$. This step generates three subfractions:

- light myelin (low-density myelin), collected from the 0.32/0.62 M sucrose interface, 
- medium density myelin, collected from the 0.62/0.7 M sucrose interface, and

- heavy myelin (high-density myelin), collected from $0.7 / 1 \mathrm{M}$ sucrose interface.

The fractions were diluted with $\mathrm{H}_{2} \mathrm{O}$ and pelleted by centrifugation at $75000 \mathrm{~g}$ for $30 \mathrm{~min}$. The pellets were washed once with $\mathrm{H}_{2} \mathrm{O}$ and recovered by $10 \mathrm{~min}$ centrifugation at $12000 \mathrm{~g}$ as described above. Myelin subfractions were resuspended in $\mathrm{H}_{2} \mathrm{O}$ or in PBS-PI. After preparation, myelin fractions were stored at $-20^{\circ} \mathrm{C}$ until the measurements were performed.

These protocols were also used to fractionate myelin-like membranes produced by primary oligodendrocytes in culture. Membrane subfractions, both separated from myelin and from cultured oligodendrocytes (pOLs), were further subjected to detergent solubility tests, immunoblotting, lipid analysis, Laurdan and FCS measurements.

\section{Lipid Extraction}

Lipids from myelin and from total membrane fractions of pOLs were prepared by chloroform-methanol extraction according to [286]. In this extraction procedure, chloroform, methanol and saline solution achieve 8:4:3 ratio in the final mixture. In brief, $350 \mu \mathrm{L}$ of samples (in a dilute aqueous salt solution) were mixed with $1050 \mu \mathrm{L}$ chloroform/methanol (2:1), shaken vigorously and the phases were separated by centrifugation at $6000 \mathrm{rpm}$ for $5 \mathrm{~min}$ in a bench-top centrifuge $5417 \mathrm{R}$ (Eppendorf AG, Hamburg, Germany). The lower phase was collected, mixed with $70 \mu \mathrm{L}$ methanol and $475 \mu \mathrm{L} 50 \mathrm{mM} \mathrm{NaCl}$ in $\mathrm{H}_{2} \mathrm{O}$, shaken and separated by centrifugation at $6000 \mathrm{rpm}$ for $5 \mathrm{~min}$. The lower phase containing lipids was speed-vacuum dried in vacuum concentrator Eppendorf Vacufuge (Eppendorf AG, Hamburg, Germany).

\section{Determination of Protein Concentration}

Protein concentration in myelin preparations and in brain homogenates was determined with Bio-Rad Protein Assay (Bio-Rad Laboratories GmbH, München, Ger- 
many), based on the method of M. Bradford [287]. The Bio-Rad Protein Assay was performed according to the manufacturer's instructions. It involves addition of an acidic dye to protein solution, and subsequent measurement of absorbance at $595 \mathrm{~nm}$. The absorbance maximum for an acidic solution of Coomassie Brilliant Blue G-250 dye shifts from $465 \mathrm{~nm}$ to $595 \mathrm{~nm}$ when binding to protein occurs. The Coomassie blue dye binds to primarily basic and aromatic amino acid residues, especially arginine. Absorbance for several dilutions of protein standard (BSA $50 \mu \mathrm{g} / \mathrm{ml}$ to $500 \mu \mathrm{g} / \mathrm{ml}$ ) and experimental probes was measured, and the Beer-Lambert law was applied for quantification of protein concentration in the samples [250]:

$$
A=\epsilon l c,
$$

where $A$ is the measured absorbance at a particular wavelength, $\epsilon$ is a molar absorption coefficient, $l$ is the path length (typically $1 \mathrm{~cm}$ for a cuvette), and $c$ is the analyte concentration.

Samples containing SDS (e.g.. cell lysates prepared as described in Section 2.2.2) were analyzed with Pierce BCA Protein Assay, which is a detergent-compatible formulation based on bicinchoninic acid (BCA). This method combines the reduction of $\mathrm{Cu}(+\mathrm{II})$ to $\mathrm{Cu}(+\mathrm{I})$ by protein in an alkaline medium (the biuret reaction) with the colorimetric detection of the monovalent cuprous cation with BCA [288]. The absorbance of the purple-colored reaction product was measured at $562 \mathrm{~nm}$ over a working range of protein concentration $20-2000 \mu \mathrm{g} / \mathrm{mL}$.

\section{Detergent Solubility Assays}

To analyze lateral microcomposition of oligodendroglial membranes, we performed detergent extractions with CHAPS as described in [289].

For detergent solubility analysis of proteins in myelin membranes, myelin preparations (1 - $10 \mu \mathrm{g}$ of protein) were incubated in of $20 \mathrm{mM}$ CHAPS in PBS (extraction buffer) supplemented with protease inhibitors (see Section 2.1.4) for $30 \mathrm{~min}$ at $4^{\circ} \mathrm{C}$ or at RT. For detergent solubility tests of proteins from cultured cells, the cells were harvested and homogenized in non-denaturating conditions ("native suspension", as described in Section 2.2.2), incubations with detergent were performed according to the same protocol as described for myelin preparations. 
Detergent-extracted samples were further separated into fractions corresponding to different detergent solubility. For crude separation of detergent-soluble and detergent-insoluble contents, the samples were centrifuged at $13000 \mathrm{~g}$ for $30 \mathrm{~min}$ at $4^{\circ} \mathrm{C}$ in a table-top cooling Eppendorf $5417 \mathrm{R}$ centrifuge (Eppendorf AG, Hamburg, Germany) [290]. Detergent-soluble fractions were collected from the supernatant, detergent-insoluble pellets were resuspended in the lysis buffer supplemented with protease inhibitors (see Section 2.2.2), in a volume equal to the volume of the soluble fractions. The samples were further analyzed by SDS-PAGE and Western blotting.

For density gradient separation of detergent-extracted samples, discontinuous gradients were prepared with different dilutions of Iodixanol (Optiprep, Nycomed Pharma, Hamburg, Germany) as described in [130]. After detergent extraction, $250 \mu \mathrm{L}$ of the lysates were adjusted to $40 \%$ Optiprep by adding $500 \mu \mathrm{L}$ of Optiprep solution (60\%), overlaid with $1.2 \mathrm{~mL}$ of $30 \%$ Optiprep in extraction buffer, and $200 \mu \mathrm{L}$ of extraction buffer. The samples were centrifuged for $2 \mathrm{~h}$ at $55000 \mathrm{rpm}$ in a TLS 55 rotor (Beckman Coulter GmbH, Krefeld, Germany) and six fractions of equal volume were collected from the top. Equal volumes of each fraction were either directly processed further for SDS-PAGE, or fractions were concentrated by adding an equal volume of $20 \%$ TCA, centrifuging for $30 \mathrm{~min}$ at $15000 \mathrm{~g}$ at $4^{\circ} \mathrm{C}$, and washing the pellets with acetone at $-20^{\circ} \mathrm{C}$.

\section{Lipid Analysis}

For analysis of lipid classes and lipid molecular species, membrane fractions of different densities were isolated from purified WT CNS myelin and from cultured primary oligodendrocytes $(24-40 \times 5 \mathrm{~cm}$ dishes with 7 days old pOLs were used per preparation), as described in Section 2.2.2. For lipid analysis of Oli-neu membranes, isolation of total membranes from $20 \times 10 \mathrm{~cm}$ dishes of cultured Oli-neu was performed as documented in Section 2.2.2. The samples (approx. $100 \mu \mathrm{g}$ of protein) were prepared in 20 - $50 \mathrm{mM}$ Tris/HCl, $\mathrm{pH} 7.4$ - 7.6. Measurements of relative abundance of lipid classes and their acyl chain composition was carried out by nano-electrospray ionization tandem mass spectrometry (nano-ESI-MS/MS) by B. Brügger at Biochemistry Center of Heidelberg University, Heidelberg, Germany, as described in [291, 292]. 


\section{Fluorescence Correlation Spectroscopy (FCS)}

In order to study the lateral organization of myelin membrane, we employed fluorescence correlation spectroscopy (FCS). FCS is a technique based on the statistical analysis of the signal fluctuations detected from fluorophores in a very small volume [293]. To estimate translational diffusion coefficients in wild-type (WT) and mutant myelin with FCS, crude myelin fractions were prepared from the brains of WT, Shiverer and CerS2 KO mice (see Section 2.2.2) followed by the lipid extraction procedure performed as described above (see Section 2.2.2). FCS measurements were carried out by S. Chiantia at Biotechnology Center, University of Technology Dresden, Germany, according to [294, 295].

\section{Generation of Giant Unilamellar Vesicles (GUVs)}

To probe the self-organization properties of myelin lipids, in vitro experiments in model membranes were performed. For this, crude and purified myelin fractions were isolated from the brains of wild-type and mutant mice (see Section 2.2.2). In addition, total membrane fractions were prepared from Oli-neu cells (see Section 2.2.2). Lipids were extracted from the samples as described in Section 2.2.2. GUVs were reconstituted from the myelin lipids, Oli-neu lipids and from the lipid extracts, mixed in 1:1 ratio. GUV preparation using Pt wires was carried out by $\mathrm{N}$. Kahya at the University Medical Center, Groningen, Netherlands, according to the protocol detailed in [294].

\section{Membrane Order Analysis with C-Laurdan}

Fluorescence spectroscopy with a C-Laurdan probe was performed to analyze membrane order in fractions of different densities isolated from purified CNS myelin and from cultured pOLs, as well as in crude myelin preparations from the brains of the wild-type and mutant mice (see Section 2.2.2). The samples containing approx. $100 \mu \mathrm{g}$ of protein were reconstituted in $\mathrm{H}_{2} \mathrm{O}$ and stored at $-20^{\circ} \mathrm{C}$ until the measurements were performed. The generalized polarization (GP) values were estimated by H.-J. Kaiser at the Max Planck Institute of Molecular Cell Biology and Genetics, Dresden, Germany, as described in [296]. 


\subsubsection{Cell Culture Techniques}

All cell culture incubations were carried out in humidified $37^{\circ} \mathrm{C}, 7.5 \% \mathrm{CO}_{2}$ incubators; further culture conditions are specified below. All solutions and equipment coming into contact with living cells were sterile, all media were filter sterilized with $0.22 \mu \mathrm{m}$ polyethersulfone (PES) filter (Corning Inc., Corning, NY, USA) and aseptic technique was used accordingly. Cell culture work was carried out according to security level S1 safety rules; handling of Semliki Forest virus and infected cells was performed in designated genetic laboratory areas according to S2 safety rules.

\section{Primary Oligodendrocyte Culture}

Primary cultures of oligodendrocytes were prepared from postnatal day 1 mouse brains as described previously [130, 237, 297]. In brief, cellular mixture from trypsinized mouse brains was grown in poly-L-lysine (PLL) coated flasks in Basal Medium Eagle (BME) medium supplemented with 10\% horse serum (PAA Laboratories GmbH, Pasching, Austria) and $100 \mathrm{U} / \mathrm{mL}$ each of penicillin and streptomycin. After 7 - 10 days, oligodendroglial progenitors growing on top of a layer of astrocytes were shaken off and cultured further in Super SATO medium (see further) on PLL coated dishes or round coverslips (R.Langenbrinck, Labor- und Medizintechnik, Emmendingen, Germany).

\section{Super SATO medium $(100 \mathrm{~mL})$}

$2 \mathrm{~mL}$ B-27 Supplement, 50X

$1 \mathrm{~mL}$ GlutaMAX-1 supplement, $200 \mathrm{mM}$

$1 \mathrm{~mL}$ Penicillin/Streptomycin, $5000 \mathrm{U} / 5000 \mu \mathrm{g}$

$1 \mathrm{~mL}$ Sodium pyruvate, $100 \mathrm{mM}$

$10 \mu \mathrm{L}$ Triiodothyronine (Calbiochem/Merck KGaA, Darmstadt, Germany), $5 \mathrm{mM}$ stock in ethanol

$13 \mu \mathrm{L}$ L-Thyroxine (Calbiochem/Merck KGaA, Darmstadt, Germany), $4 \mathrm{mM}$ stock in $0.26 \mathrm{~N} \mathrm{NaOH}, 25 \%$ ethanol

$1 \mathrm{~mL}$ Horse serum (PAA Laboratories GmbH, Pasching, Austria)

in Dulbecco's Modified Eagle Medium (DMEM) with $4.5 \mathrm{~g} / \mathrm{L}$ glucose 
Cells were plated at density approx. $3 \times 10^{4}$ cells $/ \mathrm{cm}^{2}\left(7 \times 10^{4}\right.$ to $10^{5}$ cells per well in a 12-well plate, approx. $3.5 \times 10^{5}$ cells per dish with a diameter $3.5 \mathrm{~cm}, 8 \times$ $10^{5}$ to $10^{6}$ cells per petri dish with a diameter of $6 \mathrm{~cm}$ ). After shake, the cells were maintained in culture for 2 - 6 days before using in experiments.

PLL (Mw>300000) coating for primary cultures: $100 \mu \mathrm{g} / \mathrm{mL}$ PLL was applied on the surfaces for $4-12 \mathrm{~h}$ at $37^{\circ} \mathrm{C}$, aspirated and washed with PBS.

\section{Growth and Maintenance of Oli-neu Cell Line}

The mouse oligodendrocyte precursor cell line Oli-neu ([298]; kindly provided by Prof. J. Trotter, Heidelberg, Germany) was cultured in SATO medium (see further) containing $5 \%$ horse serum on poly-L-lysine (PLL) coated coverslips or petri dishes. The cells were passaged 1:5 - 1:6 every 2 - 3 days when grown to approx. $80 \%$ confluence. Only the cells with passage number less than 35 were used for experiments.

PLL (Mw>300000) coating for cell lines: $33 \mu \mathrm{g} / \mathrm{mL}$ PLL was applied on the surfaces for $1 \mathrm{~h}$ at RT, aspirated, washed with $\mathrm{H}_{2} \mathrm{O}$ and dried.

\section{SATO medium $(100 \mathrm{ml})$}

$1 \mathrm{~mL}$ Insulin-Transferrin-Selenium-A Supplement ITS-A, 100X

$1 \mathrm{~mL}$ Putrescine dihydrochloride, stock $10 \mathrm{mM}$ in DMEM

$10 \mu \mathrm{L}$ Progesterone, stock $2 \mathrm{mM}$ in ethanol

$10 \mu \mathrm{L}$ Triiodothyronine ( Calbiochem/Merck KGaA, Darmstadt, Germany), $5 \mathrm{mM}$ stock in ethanol

$13 \mu \mathrm{L}$ L-Thyroxine (Calbiochem/Merck KGaA, Darmstadt, Germany), $4 \mathrm{mM}$ stock in $0.26 \mathrm{~N} \mathrm{NaOH}, 25 \%$ ethanol

$1 \mathrm{~mL}$ GlutaMAX-1 supplement, $200 \mathrm{mM}$

$1 \mathrm{~mL}$ Penicillin/Streptomycin, $5000 \mathrm{U} / 5000 \mu \mathrm{g}$

$5 \mathrm{~mL}$ Horse serum (PAA Laboratories GmbH, Pasching, Austria)

in Dulbecco's Modified Eagle Medium (DMEM) with $4.5 \mathrm{~g} / \mathrm{L}$ glucose. 


\section{Growth and Maintenance of Baby Hamster Kidney Cell Line (BHK21)}

BHK21 cell line was used for in vivo packaging of Semliki Forest virus particles. BHK cells were cultured in Dulbecco's modified Eagle medium (DMEM) with $4.5 \mathrm{~g} / \mathrm{L}$ glucose supplemented with 5\% fetal calf serum (FCS), 10\% tryptose phosphate broth (Sigma-Aldrich Chemie GmbH, München, Germany), 2 mM GlutaMAX-1 supplement and $100 \mathrm{U} / \mathrm{mL}$ each of penicillin and streptomycin. Cells were grown to $90 \%$ confluence on culture dishes and passaged 1:20 by detaching them from the plates using $0.05 \%$ trypsin/EDTA (Lonza GmbH, Wuppertal, Germany).

\section{MAG513 Antibody Production with Hybridoma Cell Line}

To produce large amounts of antibodies immunoreactive to myelin-associated glycoprotein (MAG), we cultured MAG513 hybridoma cell line, kindly provided by Dr. Geoffrey Owens, The Neuroscience Institute, San Diego, CA [235].

First, the cells were amplified in high-serum RPMi+ growth medium (specified below). The cells were further passaged 1:1 with reducing serum content in each passaging step by diluting RPMi+ medium twice with RPMi serum-reducing medium. After 3 serum-reducing steps, the serum content reached $1.25 \%$, which is necessary for induction of antibody production. Cell growth medium containing immunoglobulins was harvested after $24 \mathrm{~h}$ incubation of hybridoma cells in medium with $1.25 \%$ serum. The medium was cleared by centrifugation and the supernatant containing anti-MAG antibodies was aliquoted, snap-frozen in liquid nitrogen and stored at $-20^{\circ} \mathrm{C}$ until further use for detection of MAG epitopes in immunoblotting.

\section{Hybridoma growth media RPMi+ $(500 \mathrm{~mL})$}

$5 \mathrm{~mL}$ GlutaMAX-1 supplement, $200 \mathrm{mM}$

$5 \mathrm{~mL}$ Penicillin/Streptomycin $5000 \mathrm{U} / 5000 \mu \mathrm{g}$

$50 \mathrm{~mL}$ Fetal calf serum (FCS)

in RPMi (Roswell Park Memorial Institute) media. 


\section{RPMi serum-reducing media $(500 \mathrm{~mL})$}

$5 \mathrm{~mL}$ GlutaMAX-1 supplement, $200 \mathrm{mM}$

$5 \mathrm{~mL}$ Penicillin/Streptomycin $5000 \mathrm{U} / 5000 \mu \mathrm{g}$

$5 \mathrm{~mL}$ Insulin-Transferrin-Selenium-X Supplement ITS-X 100X

in RPMi (Roswell Park Memorial Institute) media.

\section{Cryopreservation, Storage and Thawing of Cell Lines}

For long-term storage of cell lines, cells were grown to maximum confluence, detached from the surfaces according to the cell type (with trypsin/EDTA, if necessary) and pelleted by centrifugation at $900 \mathrm{~g}$ for $5 \mathrm{~min}$. The cells were gently resuspended in $1 \mathrm{ml}$ of corresponding basal medium, supplemented with $20 \%$ FCS, 10\% DMSO and transferred into Nalgene® Sterile Cryogenic Vials (Thermo Fisher Scientific, Roskilde, Denmark). Slow freezing was carried over night in $-80^{\circ} \mathrm{C}$ freezer in a Nalgene $\AA$ Cryo $1^{\circ} \mathrm{C}$ Freezing Container (Thermo Fisher Scientific, Roskilde, Denmark) or a polystyrene block before permanent storage in liquid nitrogen. To thaw cryopreserved cells, cells in cryovials were removed from the storage tank and immediately immersed in a $37^{\circ} \mathrm{C}$ water bath for $1 \mathrm{~min}$. The cell suspension was added to $9 \mathrm{~mL}$ of fresh growth medium, pelleted with centrifugation at $900 \mathrm{~g}$ for 5 min. The cells were gently resuspended in fresh medium and transferred into a $10 \mathrm{~cm}$ petri dish or a $75 \mathrm{~cm}^{2}$ cell culture ask with coating, if necessary. The cells were further grown and passaged normally according to the cell type, as described in previous sections.

\section{Methods for Nucleic Acids Delivery into Cultured Animal Cells}

To introduce exogenous nucleic acids into cultured cells we used lipofection-based and electroporation-based methods of transient transfection, as well as virus-mediated gene transfer (transduction).

Lipofection-based transient transfection. This method utilizes the ability of lipid vesicles (liposomes), encapsulating or binding nucleic acids, to fuse with cellular membranes and thus introduce DNA or RNA into cells [299, 300]. 
To deliver expression vectors into cultured Oli-neu cells, FuGENE@ 6 (Roche Diagnostics GmbH, Mannheim, Germany) or TransITß) (Mirus Bio LLC, Madison, WI, USA) transfection reagents were used. The cells were cultured to approx. $75 \%$ confluence on the day of transfection. For each coverslip in a 12-well plate, $3 \mu \mathrm{L}$ of transfection reagent and $1 \mu \mathrm{g}$ of plasmid DNA were added subsequently to OptiMEM-I (Invitrogen, Carlsbad, CA, USA), gently mixed and incubated at RT for $30 \mathrm{~min}$. The mixture was added dropwise to the cells; the cells were incubated further under normal cultural condition and analyzed after $12-18 \mathrm{~h}$.

After testing various transfection reagents, we chose Lipofectamine $\mathbb{R} 2000$ (Invitrogen GmbH, Darmstadt, Germany) for transfection of primary oligodendrocytes. Transfections were performed on day 3 or 4 after shake; primary oligodendrocytes prepared for transfection were grown in Super SATO medium without antibiotics. Per each coverslip in 12-well plates, 2 mixtures were set: in one testtube, $1.6 \mu \mathrm{g}$ of purified plasmid DNA was mixed with $100 \mu \mathrm{L}$ OptiMEM-I (Invitrogen, Carlsbad,

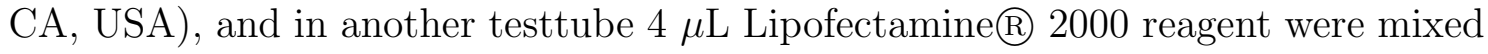
with $100 \mu \mathrm{L}$ OptiMEM and left for $5 \mathrm{~min}$ at RT. Afterwards, the lipofectaminesolution and the DNA-solution were combined and incubated for $30 \mathrm{~min}$ at RT. The total volume of $200 \mu \mathrm{L}$ lipofectamine-DNA-mixture was added dropwise to each coverslip. After 4 - $6 \mathrm{~h}$, the medium was exchanged to fresh SuperSato medium (see Section 2.2.3) and the cells were analyzed 12 - $18 \mathrm{~h}$ later.

When transfections with FuGENE@, TransITß or Lipofectamine® 2000 were required to be performed in culture vessels, different from 12-well plates, the amounts of DNA and transfection reagents were recalculated according to the surface area of the culture vessel.

Electroporation. For knock-down experiments, siRNAs were delivered into cells by electroporation with Basic Neuron Nucleofector Kit (Lonza Group Ltd, Basel, Switzerland). This technique is based on application of brief, high-voltage electric pulses which lead to the formation of nanometer-sized pores and introduction of nucleic acids from medium into cellular cytoplasm [301], or in case of Nucleofection technology, directly into nuclei.

$2 \times 10^{6}$ cells were pelleted by centrifugation at $900 \mathrm{~g}$ for $5 \mathrm{~min}$ and resuspended in $100 \mu \mathrm{L}$ Neucleofector solution. $160 \mathrm{pmol}$ of siRNA was added to the suspension and 
delivered into the cells with electroporation program O-05 in Amaxa Nucleofector (Lonza Group Ltd, Basel, Switzerland). The cells were then transferred to a precoated $10 \mathrm{~cm}$ dish (coverslips or $3 \mathrm{~cm}$ dishes in case of primary cells) with prewarmed fresh growth medium without antibiotics. After 3 days, Oli-neu cells were electroporated again as described above. The cells were resuspended in fresh medium and plated into culture vessels at a density of $1.5 \times 10^{5}$ cells/well in 12 -well plate or $6 \times 10^{5}$ cells per $3 \mathrm{~cm}$ dish. The cells were collected for analysis 3 days after the first or second electroporation.

RNA electroporation into BHK21 cells was performed as detailed in [270].

Transduction. We found virus-mediated gene transfer being currently the most efficient way to transiently deliver transgenes into primary oligodendrocytes. Conditions for SFV infections of primary oligodendrocytes are described in Section 2.2.1.

\section{Immunocytochemistry}

To analyze the subcellular localization of the proteins and lipids of interest in primary oligodendrocytes and Oli-neu cells we performed immunocytochemistry as described in [302]. In brief, cells on coverslips were fixed for $10 \mathrm{~min}$ at RT with $4 \%$ paraformaldehyde (PFA) in PBS, pH 7.4, followed by three washing steps in PBS. If required, cells were permeabilized with $0.1 \%$ Triton X-100 for $1 \mathrm{~min}$ at RT or on ice for $30 \mathrm{~s}$ for filipin stainings, then washed 3 times in PBS. To reduce unspecific binding of antibodies, blocking was carried out for 10 min at RT in $2 \%$ FCS, $2 \%$ BSA and $2 \%$ Fishgelatine in PBS (100\% blocking solution). The cells were incubated with the primary antibodies in appropriate dilution in $10 \%$ blocking solution for $1 \mathrm{~h}$ at RT, washed three times for 5 min with PBS, incubated with the secondary antibodies in required dilution in $10 \%$ blocking solution, washed three times in PBS and mounted in Mowiol mounting medium. The list of antibodies used can be found in Table 2.1.

Preparation of PFA. To prepare PFA 16\% stock solution, $16 \mathrm{~g}$ PFA were heated at $60^{\circ} \mathrm{C}$ in approx. $70 \mathrm{~mL} \mathrm{H} \mathrm{H}_{2} \mathrm{O}, \mathrm{NaOH}$ pellets were added to facilitate solubilising. $10 \mathrm{~mL}$ 10X PBS were added to the solution, $\mathrm{pH}$ was adjusted to 7.4 and the total volume was adjusted to $100 \mathrm{~mL}$. The solution was aliquoted and stored 
at $-20^{\circ} \mathrm{C}$. To prepare $4 \% \mathrm{PFA}$ in PBS, $50 \mathrm{~mL}$ of PFA stock solution was diluted with $150 \mathrm{~mL}$ of $1 \mathrm{X}$ PBS. The solution was stored at $4^{\circ} \mathrm{C}$ and prewarmed to RT before use.

Preparation of Mowiol. To prepare Mowiol mounting medium, $2.4 \mathrm{~g}$ Mowiol (Calbiochem/Merck KGaA, Darmstadt, Germany), 6 g glycerol, $6 \mathrm{~mL} \mathrm{H}_{2} \mathrm{O}$ were mixed and incubated at RT for $2 \mathrm{~h}$ with agitation. After addition of $12 \mathrm{~mL} 0.2 \mathrm{M}$ Tris $/ \mathrm{HCl}(\mathrm{pH} 8.5)$, the mixture was heated at $50^{\circ} \mathrm{C}$ for $10 \mathrm{~min}$. The solution was cleared by centrifugation at $5000 \mathrm{~g}$ for $15 \mathrm{~min}$. Anti-fading reagent DABCO was added in amount $24 \mathrm{mg}$ per $\mathrm{mL}$ of media. The solution was aliquoted and stored at $-20^{\circ} \mathrm{C}$.

\subsubsection{Microscopy, Image Processing and Statistical Analysis}

\section{Confocal Microscopy}

For morphological examination of myelin membrane sheets produced by primary oligodendrocytes we chose laser scanning confocal microscopy. This technique gives an advantage of high resolution in combination with control over depth of field, which is invaluable for the analysis of organization of thin membrane sheets.

The basic concept of confocal microscopy was originally developed and patented by M. Minsky in the middle of the $20^{\text {th }}$ century [303].

In laser scanning confocal microscopy, a focused laser beam illuminates and excites point by point small parts of a sample in one focal plane. The emitted fluorescent light passes through beam splitter (AOBS in case of Leica SP2) and then through a pinhole which rejects out-of-focus light. After passing a pinhole the fluorescent light is detected by a photomultiplier tube (PMT) transforming the light signal into an electrical one which is recorded by a computer [304]. Due to the laser light excitation and pinhole-based rejection of the off-focus light, confocal microscopy provides higher resolution images with less background than conventional light microscopy [305].

Confocal microscopic images were acquired with Leica DMIRE2 microscope and a Leica TCS SP2 AOBS confocal laser scanning setup (Leica Microsystems, Mannheim, Germany). 40X NA 1.25 or 63X NA 1.4 oil plan-apochromat objectives 
(Leica Microsystems, Mannheim, Germany) were used for image acquisition. For live cell imaging, temperature control was applied to the microscope setup (Leica Box and Leica Cube) and the cells were imaged in a modified Krebs-Ringer solution (see below, [306]) in a self-made closed chamber at $37^{\circ} \mathrm{C}$ for no longer than $1 \mathrm{~h}$.

\section{Modified Krebs-Ringer solution}

$\begin{array}{rl}120.0 \mathrm{mM} & \mathrm{NaCl} \\ 4.7 \mathrm{mM} & \mathrm{KCl} \\ 1.2 \mathrm{mM} & \mathrm{CaCl}_{2} \\ 0.7 \mathrm{mM} & \mathrm{MgSO}_{4} \\ 10.0 \mathrm{mM} & \text { Glucose } \\ 20.0 \mathrm{mM} & \text { HEPES, pH } 7.4\end{array}$

\section{Image Analysis}

Images were processed and analyzed with the public domain Java-based image processing software Image ${ }^{6}$ (created by Rasband, W.S., National Institutes of Health, Bethesda, Maryland, USA), or with an MBF collection of plugins for ImageJ (MBF "ImageJ for Microscopy"7).

To estimate colocalization of two proteins by immunocytochemistry, both proteins were detected with antibodies coupled to distinguishable fluorophores. Each color channel was imaged separately, and the Pearson's correlation coefficient between the two channels was calculated with Intensity Correlation Analysis ImageJ plugin [307]. Pearson's correlation coefficient $R_{x y}$ between two channels (images) $x$ and $y$ was calculated with the following equation:

$$
R_{x y}=\sum_{i=1}^{n} \frac{\left(x_{i}-\bar{x}\right)\left(y_{i}-\bar{y}\right)}{\sqrt{\sum_{i=1}^{n}\left(x_{i}-\bar{x}\right)^{2} \sum_{i=1}^{n}\left(y_{i}-\bar{y}\right)^{2}}},
$$

where $x_{i}$ and $y_{i}$ are the values of pixel $i$ in channels $x$ and $y$.

Values for Pearson's correlation coefficient range from 1 to -1 ; values close to 1 are indicative of reliable colocalisation in image analysis [304]. For colocalization analysis, 8-bit confocal images, $1024 \times 1024$ pixels were acquired. To facilitate the

\footnotetext{
${ }^{6}$ http://rsb.info.nih.gov/ij/

${ }^{7}$ http://www.macbiophotonics.ca/imagej/index.htm
} 
analysis, a self-written macro utilizing Intensity Correlation Analysis ImageJ plugin for Pearson's correlation calculations was used (see codes in Appendix B).

Quantification of relative cell surface area occupied by the myelin proteins in MBP-deficient (Shiverer) and wild type (WT) oligodendrocytes was performed using the following options in ImageJ: application of threshold from background (with the Segmentation Plugin to avoid user-bias from manual setting of the threshold [308]), defining cell area (Freehand selection tool), followed by measurement of the area fraction, occupied by the signal (Set measurements - Area fraction - Measure).

Western blot densitometric analysis was carried out on 600 dpi blot scans with the ImageJ Gel Analyser (Analyse - Gels - Select lanes - Plot lanes - Measure peak areas).

\section{Atomic Force Microscopy (AFM)}

To estimate the thickness of the membrane sheets developed by primary oligodendrocytes, atomic force microscopy (AFM) experiments were carried out. Primary oligodendrocytes used for measurements were cultured for 4 days after shake on $25 \mathrm{~mm}$ diameter glass coverslips for AFM, fixed, permeabilized, stained for CNPase and MBP as described in Section 2.2.3 and subjected to AFM recordings in PBS (see Section 2.1.4). AFM measurements were performed by J. Friedrichs and N. Strohmeyer in D. Müller's group at Biotechnology Center, University of Technology Dresden, Germany [309, 310].

\section{Fluorescence Recovery After Photobleaching (FRAP)}

To evaluate lateral mobility processes in plasma membranes of oligodendroglial cells, we employed fluorescence recovery after photobleaching (FRAP) technique. In the FRAP approach, an intense, focused laser pulse of appropriate wavelength bleaches a small area on the fluorescently labeled membrane (or other entity) [311, 312]. Subsequently, the reappearance of fluorescence within this area due to the arrival (via diffusion and/or flow) of unbleached molecules is monitored. The mobility characteristics of the labeled component can be estimated from the analysis of their fluorescence recovery curves (fluorescence intensity vs. time after bleaching) [313]. 
For FRAP experiments, plasma membranes of primary oligodendrocytes were fluorescently labeled either with Cell Mask Orange lipofilic dye (Invitrogen GmbH, Darmstadt, Germany) or GalC monoclonal antibodies coupled to anti-mouse IgG Fab fragments, Rhodamine labeled. Cells were imaged with a confocal laser scanning microscope Leica TCS SP2 with a 60X NA 1.4 oil objective; the pinhole was set at 1 Airy unit. Photobleaching was performed in 4 scans with 561 laser at full power within a rectangular region $5 \times 5 \mu \mathrm{m}$ (zoom-in mode). Pre- and postbleach fluorescence intensities (2 and 20 scans respectively) were monitored with approx. $25 \%$ laser power. 8-bit images were recorded every 0.657 s at resolution $512 \times 512$ pixels, with scanner speed $800 \mathrm{~Hz}$.

Processing of FRAP data was based on [313, 314]: In FRAP experiments, for each cell, 3 areas were specified, for which the intensity values were collected: (1) bleached region of interest (ROI, $5 \times 5 \mu \mathrm{m}$ ), (2) cell area, distant of the bleach area, (3) background area. Data were processed in following steps:

\section{Background subtraction}

Average background intensity was subtracted from ROI intensity values and from intensity values of cell area distant of the bleach area:

$$
\begin{gathered}
F^{\prime}(t) R O I=F(t) R O I-F(t) b k g d \\
F^{\prime}(t) c e l l=F(t) c e l l-F(t) b k g d
\end{gathered}
$$

where $F(t)$ is an intensity value at each time point,

$t$ is the time for each intensity value,

$F(t) R O I$ is the bleach ROI intensity at each time point,

$F^{\prime}(t) R O I$ is the background corrected bleach ROI intensity at each time point, $F(t) b k g d$ is the intensity of background fluorescence at each time point, $F(t)$ cell is the cell fluorescence intensity distant of the bleach area at each time point,

$F^{\prime}(t)$ cell is the background corrected cell fluorescence intensity distant of the bleach area at each time point. 


\section{Correction for loss of fluorescence due to photobleaching}

To correct the intensities for unintended sample bleaching during acquisition of post-bleach images, the following equation was used:

$$
F^{\prime \prime}(t) R O I=F^{\prime}(t) R O I \times \frac{F^{\prime}(0) c e l l}{F^{\prime}(t) c e l l},
$$

where $F^{\prime \prime}(t) R O I$ is the background and photobleach corrected bleach ROI intensity at each time point,

$F^{\prime}(0)$ cell is the cell initial fluorescence intensity (before bleaching and acquisition of post-bleach images), background corrected.

\section{Normalization}

To normalize the pre-bleach ROI intensities to 1, every frame (time point) is divided by the first frame for the ROI:

$$
F^{\prime \prime \prime}(t) R O I=\frac{F^{\prime \prime}(t) R O I}{F^{\prime \prime}(0) R O I}
$$

where $F^{\prime \prime}(0) R O I$ is the mean of the initial intensities of the ROI (before bleaching), background and photobleach corrected,

$F^{\prime \prime \prime}(t) R O I$ is the background and photobleach corrected normalized ROI intensity at each time point.

\section{Curve fitting}

Normalized data were fitted to a single exponential curve:

$$
f(x)=a\left(1-e^{-b x}\right)+y_{0},
$$

using the SigmaPlot software (Systat Software GmbH, Erkrath, Germany). Mobile fraction $\left(M_{f}\right)$ is a fraction of fluorescent molecules that recover in a bleached area during the time-course of the experiment. $M_{f}$ was calculated from the equation parameters of the fitted curve:

$$
M_{f}=a+y_{0} .
$$

To derive recovery half-time $\left(t_{1 / 2}\right)$, which indicates the time at which half of the fluorescence has recovered, the following calculation was done:

$$
t_{1 / 2}=\ln 2 / b
$$


Diffusion coefficients $(D)$ can be estimated based on the formula proposed by D. Axelrod and D. Soumpasis [311, 312] for two-dimensional diffusion:

$$
D=0.224 \times w^{2} / t_{1 / 2}
$$

where $w$ is the radius of the circular bleach area.

\section{Total Internal Reflection Fluorescence (TIRF) Microscopy}

To determine endocytosis sites in cultured primary oligodendrocytes, we employed TIRF microscopy. The principle of total internal reflection fluorescence microscopy is based on the excitation of fluorophores by an evanescent field of totally internally reflected light [315]. The evanescent wave arises at the interface between two media with a high (glass coverslip) and a low refractive index (cell adherent to the coverslip). The strength of the evanescent field decreases exponentially (penetration depth approx. $150 \mathrm{~nm}$ ) allowing selective excitation of fluorophores in a cell region adherent to a glass coverslip. We designed and cloned pHluorin-MOG, pHluorinMAG and pHluorin-PLPTM4 constructs (see Appendix A) to map the incorporation sites of these proteins into the plasma membrane of oligodendrocytes.

\section{Statistical Analysis}

Statistical evaluation of results was performed using SigmaPlot 11.0 software (Systat Software Inc., San Jose, CA, USA). To describe the data with basic statistics, the means (an average value for a data set) and the standard deviations (a measure of data variability about the mean) were calculated. For comparisons of two groups, the unpaired t-test was chosen if the samples were taken from normally distributed populations and the variances of the two populations were equal [316]. For populations of non-normal distribution and for populations with unequal variances, the nonparametric Mann-Whitney Rank Sum Test was used. Comparisons of several groups were carried out with One Way Analysis of Variance (ANOVA), a parametric test that compares the effect of a single factor on the means of two or more groups. For data which fail either normality or equal variance tests, the nonparametric Kruskal-Wallis ANOVA on Ranks was performed. If the differences between 
the groups could be detected with ANOVA, an appropriate multiple comparison test was further applied (Tukey test or Dunn's test) to determine which groups were different. Conclusions about significant differences were drawn when the $p$ values were less than 0.05 . 


\section{Chapter 3}

\section{Results}

\subsection{Characterization of the Cellular Model System}

This study focuses on molecular aspects of myelin membrane assembly and expansion. In vivo, myelin is an insulating multilayer of plasma membrane, which is spirally wrapped around axons. As visualization of membrane trafficking in the tightly packed, nanometer-scaled layers is an extremely challenging task, we have established a 2D model of the myelin sheath. In this model system, mouse primary oligodendrocytes (pOLs) are grown on coverslips and their processes and plasma membrane sheets can be easily observed and analyzed at all stages of their development in culture. First, we characterized the cellular model system in order to assess whether cultured oligodendrocytes are suitable for addressing the aforementioned questions.

\subsubsection{Immunocytochemical Localization of Myelin-Specific Proteins and Lipids in pOLs}

In this Section, the morphological characterization of the plasma membrane extensions developed by cultured oligodendrocytes is described. Morphological analysis was performed using immunocytochemistry in conjunction with laser scanning confocal microscopy.

With respect to pOLs morphology, we distinguished two major subcellular domains: (1) cell body together with the processes and (2) thin membrane expansions 


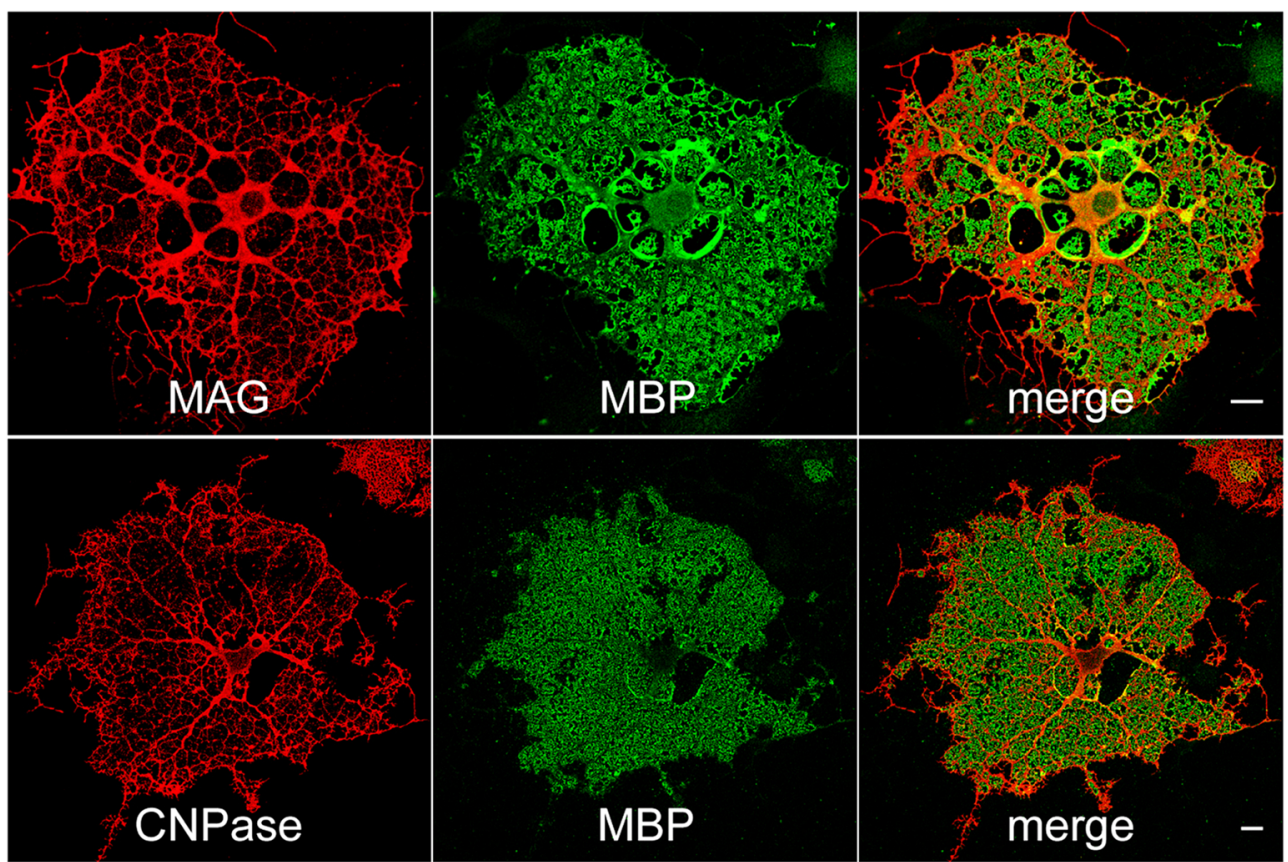

Figure 3.1: Immunocytochemical localization of the non-compact myelin proteins MAG and CNPase relative to the compact myelin protein MBP in pOLs.

Mouse primary oligodendrocytes were immunostained with antibodies specific for the non-compact myelin proteins MAG (red) and CNPase (red) and antibodies against the reference compact myelin protein MBP (green), which highlights myelin-like membrane sheets. The overlay (merge) images show that the non-compact myelin proteins are predominantly localized in the cell body and processes of oligodendrocytes, and are depleted from the MBP-enriched membrane sheets. Scale bar, $10 \mu \mathrm{m}$.

in between the processes (termed sheets further on). To assess how these domains correlate with the compact and non-compact areas myelin develops in vivo, we examined the distribution of myelin marker proteins and lipids in the processes and sheets of cultured oligodendrocytes.

Immunocytochemical analysis revealed that the processes and cell bodies of primary oligodendrocytes are enriched in 2',3'-cyclic-nucleotide 3'-phosphodiesterase (CNPase) and myelin-associated glycoprotein (MAG) (Figure 3.1).

In contrast, the sheets of mature pOLs were lacking these proteins, but contained myelin basic protein (MBP), which is a defining protein of compact myelin. Sheets of 


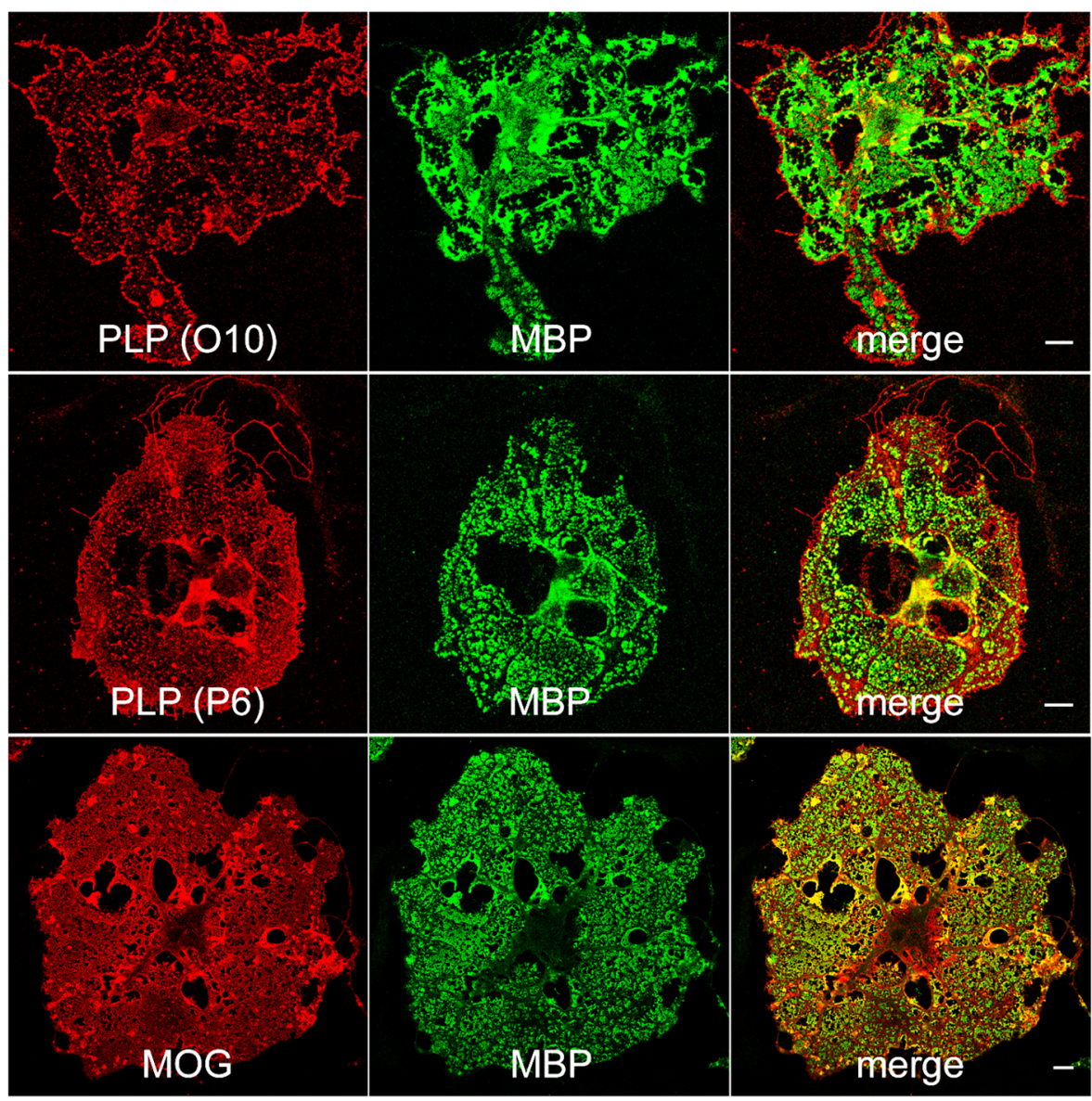

Figure 3.2: Immunocytochemical localization of the compact myelin proteins PLP and MOG in POLs.

Myelin-like membrane sheets of primary oligodendrocytes are highlighted by MBP staining (green). The surface and the total PLP pools are detected with $\mathrm{O} 10$ (upper panel, red) and P6 (middle panel, red) antibodies respectively. MOG immunoreactivity is shown in the lower panel (red). Scale bar, $10 \mu \mathrm{m}$.

pOLs were also enriched in myelin oligodendrocyte glycoprotein (MOG) (Figure 3.2), a protein confined to the outer layers of compact myelin in vivo [100].

Plasma membrane incorporated proteolipid protein (PLP) (Figure 3.2), as well as lipid galactosylceramide (GalC and O1 antibody stainings) (Figure 3.3), were distributed in the surface of oligodendrocytes, both over the sheets, as well as over the processes and cell bodies of pOLs. 


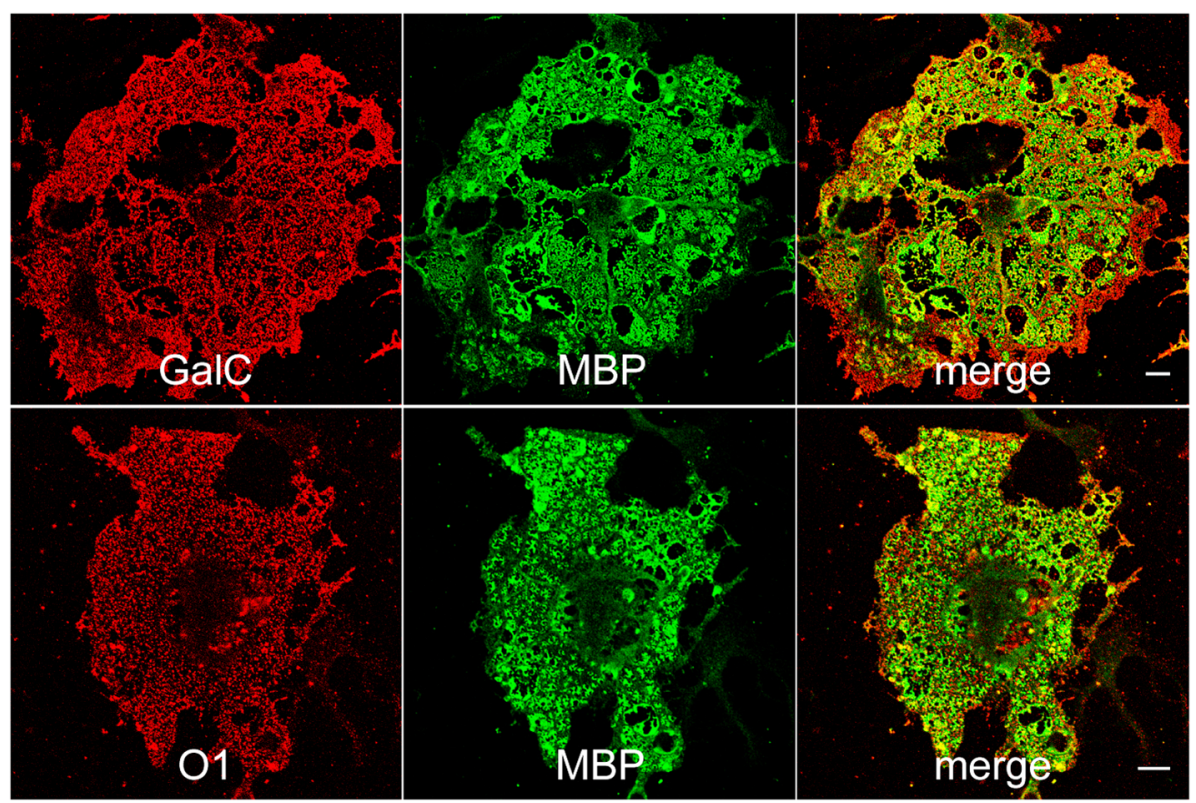

Figure 3.3: Immunocytochemical localization of galactosphingolipids in primary oligodendrocytes.

Confocal images of mouse primary oligodendrocytes immunostained for galactosylceramide (red) and for MBP (green) to resolve myelin-like membrane sheets are shown. Galactosphingolipids are detected with monoclonal mouse IgG GalC and IgM $\mathrm{O} 1$ antibodies. Overlay (merge) images demonstrate dispersed distribution of galactosylceramide epitopes on the surface of pOLs, both over the MBP-positive domains and in the processes. Scale bar, $10 \mu \mathrm{m}$.

To quantitatively describe the distribution of the myelin proteins in cultured oligodendrocytes, their colocalization with the marker protein of compact myelin MBP was analyzed. Quantitative image analysis was carried out for the following immunostainings: CNPase and MBP stainings on permeabilized cells, and the surface stainings for MAG and MOG. PLP and GalC immunostainings were not included in the analysis, since the $\mathrm{O} 1, \mathrm{O} 10$ and GalC antibodies provided a punctuate signal pattern. The degree of colocalization was assessed by calculating the Pearson's correlation coefficient between the fluorescent channels in co-stainings MAG:MBP, CNPase:MBP, MOG:MBP. The value of this coefficient ranges from -1 to 1 , with a value of -1 representing a total lack of overlap between pixels from the 
Colocalization of myelin proteins with MBP

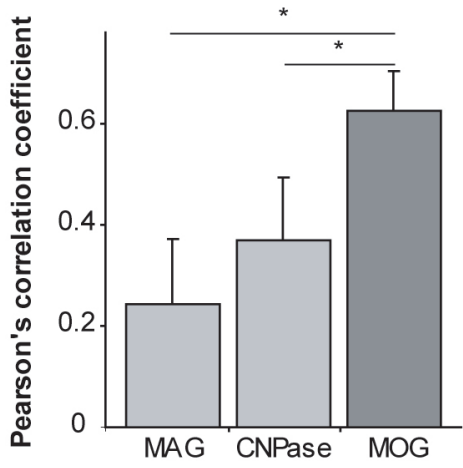

Figure 3.4: Morphological characterization of the cellular model system: quantification of colocalization of compact and non-compact myelin proteins in pOLs.

Multi-channel confocal images of pOLs immunostained for MAG, CNPase, MOG and MBP were quantified by determining the Pearson's correlation coefficient between channels with MBP signal and channels corresponding to a respective myelin protein signal. Correlation coefficient values for different myelin proteins were compared by KruskalWallis ANOVA on ranks, followed by pairwise multiple comparison procedures (Dunn's method). * denotes significantly different pairs, $\mathrm{P}<0.05 ; \mathrm{n}=18-20$. Graph bars represent mean values $\pm \mathrm{SD}$.

images, and a value of 1 indicating perfect image registration [304]. In the described immunostainings of pOLs, Pearson's correlation coefficient values were significantly lower between the compact myelin protein MBP and the non-compact myelin proteins (MAG and CNPase) than between MBP and another compact myelin protein MOG (Figure 3.4).

\subsubsection{Biochemical Separation of Myelin and pOLs Membranes into Fractions with Distinct Protein Composition}

To further characterize plasma membrane extensions produced by cultured oligodendrocytes, we employed subcellular fractionation in conjunction with Western blotting and compared the membrane fractions obtained from cultured pOLs and from myelin preparations of the CNS myelin. 


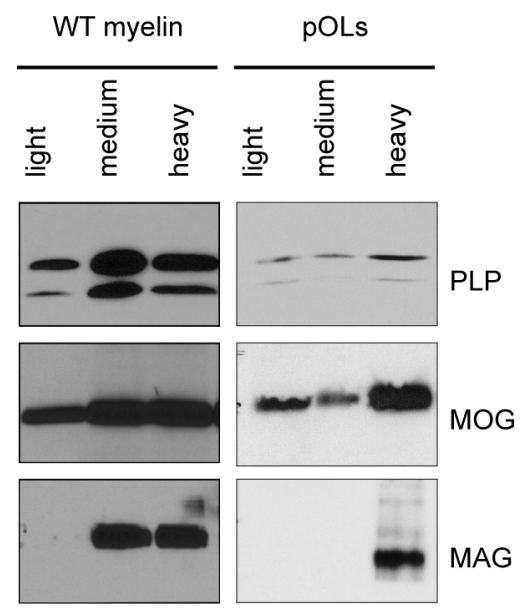

Figure 3.5: Western blot analysis of subfractions of different densities, isolated from myelin and from pOLs membranes.

Myelin from the wild-type mice (WT myelin) and membranes of cultured primary oligodendrocytes ( $\mathrm{pOLs}$ ) were subjected to biochemical fractionation by differential centrifugation in sucrose gradients. Light, medium and heavy subfractions were collected from $0.32 / 0.62,0.62 / 0.7$ and $0.7 / 1 \mathrm{M}$ sucrose interfaces respectively. Equal volumes of the fractions were resolved by SDS-PAGE and analyzed by Western blotting. Myelin proteins were detected with mouse monoclonal antibodies specific for PLP (3F4), MAG and MOG. The non-compact myelin protein MAG was enriched in the heavier membrane fractions, whereas the compact myelin proteins PLP and MOG were also found in the lighter fractions.

CNS myelin was biochemically purified from the brains of wild-type mice (NMRI, P21) by standard differential centrifugation in a sucrose gradient and further separated into subfractions of different densities (light, medium and heavy myelin). The same myelin isolation and subfractionation protocol was applied to primary cultures of oligodendrocytes (6 - 8 days after shake).

The compact myelin proteins PLP and MOG were recovered from all the fractions, whereas the non-compact myelin protein MAG predominantly associated with the fractions of higher density (heavy myelin) both in myelin subfractions and in the subfractions from cultured pOLs (Figure 3.5). Interestingly, restriction of MAG 


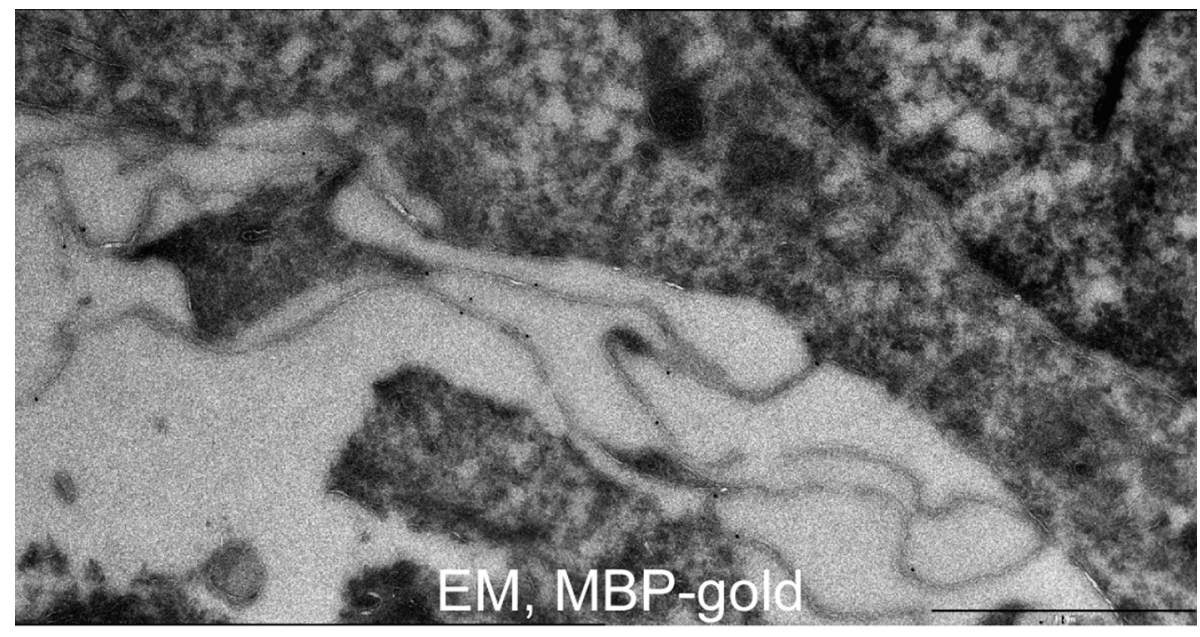

Figure 3.6: Immunoelectron microscopy (EM) of primary oligodendrocytes shows tightly compacted membrane sheaths containing MBP (electron-dense gold particles). Scale bar, $200 \mathrm{~nm}$. EM image is a courtesy of W. Moebius and S. Aggarwal.

to the heavier fractions was consistenty more profound in pOLs preparations than in subfractions obtained from myelin.

\subsubsection{Ultrastructure of Myelin Sheets in pOLs}

Examination of the ultrastructure of the myelin-like membrane sheets of primary oligodendrocytes revealed that MBP-positive membranes (detected with primary polyclonal rabbit anti-MBP antibodies and secondary immunogold antibodies) are compacted, in contrast to MBP-negative regions of the cells, containing cytoplasm.

Taken together, the performed analysis of the cellular model system revealed distinct morphological and biochemical localization of compact and non-compact myelin proteins in pOLs, similar to the patterns observed for the CNS myelin in vivo. Furthermore, characterization of primary oligodendrocytes demonstrated that in culture pOLs develop domains resembling compact and non-compact areas of myelin, which supports the adequacy of the model. 


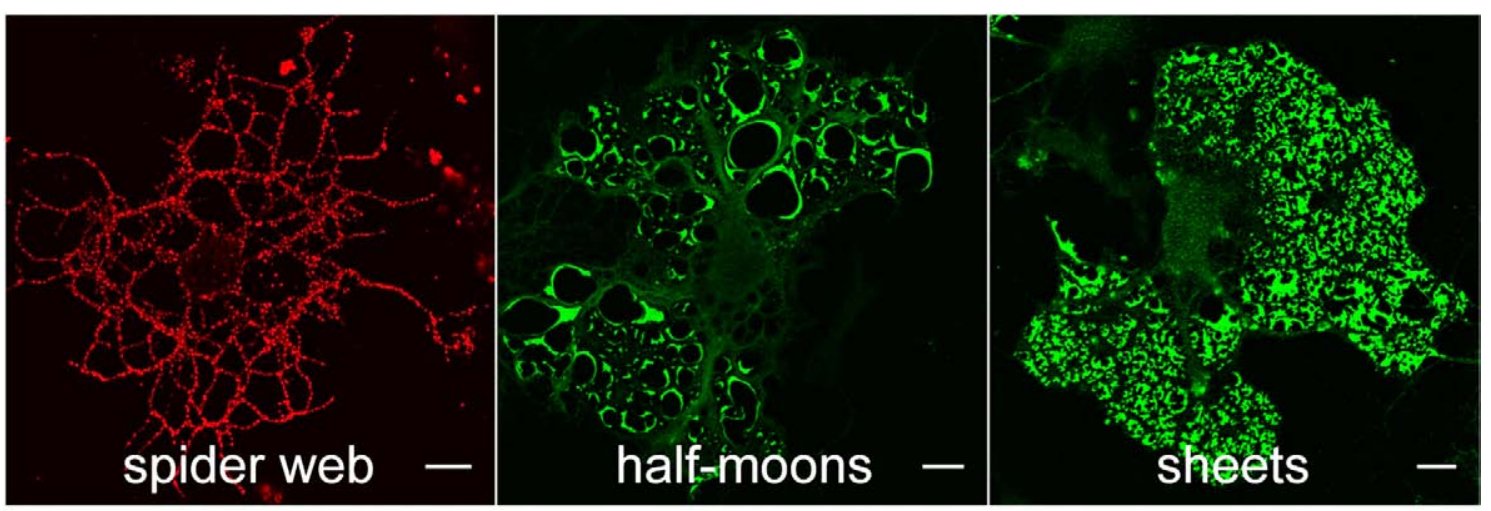

Figure 3.7: Stages of myelin sheet development: morphological changes in culture. In the first image a typical immature oligodendrocyte at a "spider web" stage is shown. The mouse oligodendrocyte precursor cells were grown on coverslips for 2 - 2.5 days after shake, fixed and then immunostained for galactosylceramide (red). These cells elaborate extensive networks of interconnected branches.

When cultured for 1 more day, oligodendrocytes progress to a "half-moon" stage, defined by a characteristic pattern of an extremely strong MBP immunostaining (bright green signal). During this rapid transition from the processes to the flat membrane sheets, MBPpositive membranes start filling up the gaps between the processes of oligodendrocytes. A mature oligodendrocyte at the "sheets" stage is presented in the last image. Within 5 days after shake most oligodendrocytes pass the "spider web" and the "half-moon" stages and extend large plasma membrane sheets. Scale bar, $10 \mu \mathrm{m}$.

\subsection{Plasma Membrane Extension in Primary Oligoden- drocytes}

\subsubsection{How are Myelin Sheets Produced During Development?}

To address the question of myelin membrane growth, we analyzed how the myelinlike membrane sheets develop during differentiation of oligodendrocytes in culture. 


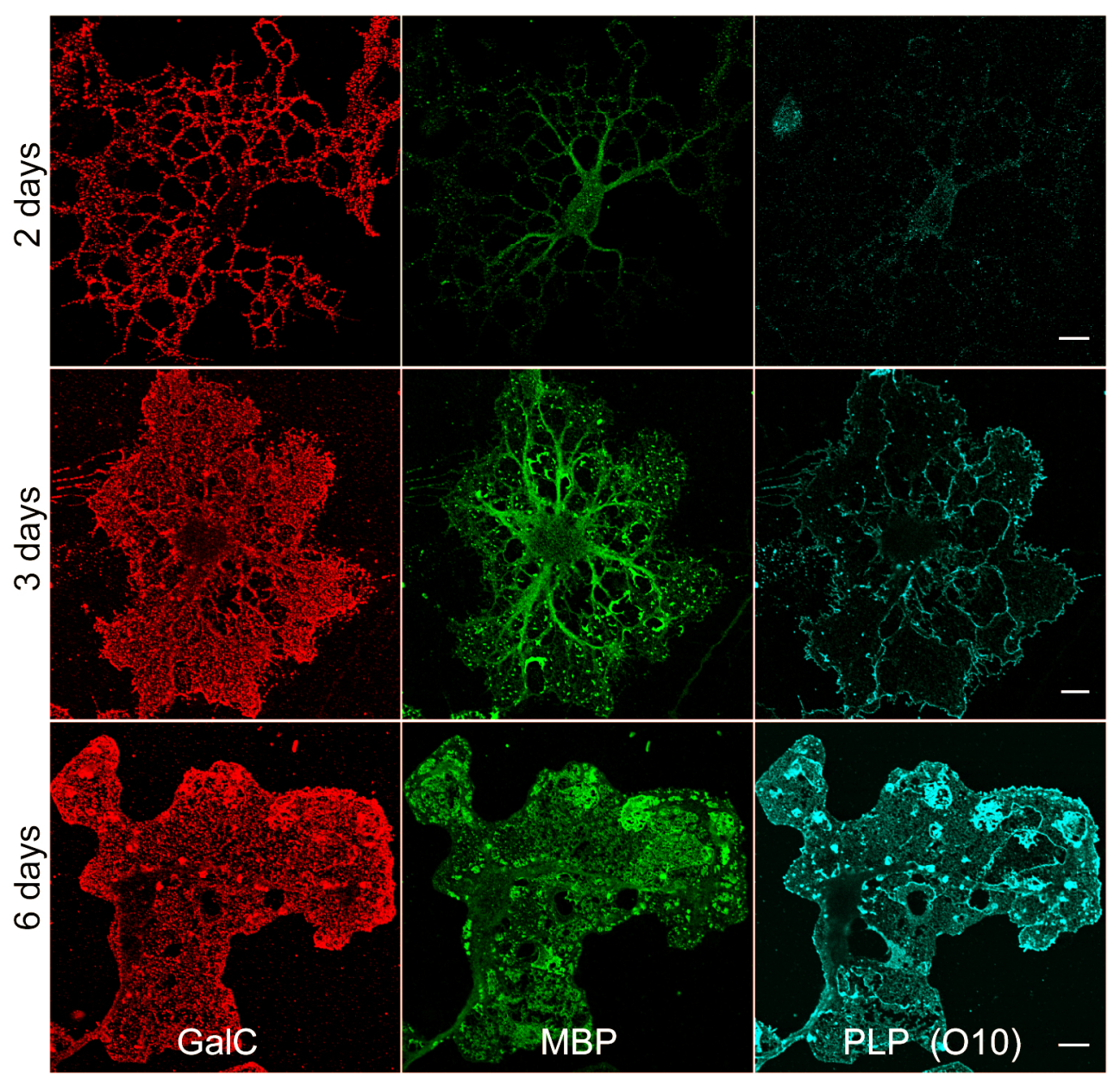

Figure 3.8: Stages of myelin sheet development: PLP incorporation into plasma membrane.

Oligodendrocytes at three developmental stages were stained with the surface antibodies against an extracellular epitope of PLP (O10, cyan) to detect the plasma membrane localization of PLP. After fixation, oligodendrocytes were labelled with GalC antibodies (red), the earliest oligodendroglial marker employed in this study, in order to detect the total pool of oligodendrocytes at various maturation stages. To facilitate discrimination of the more mature pOLs from the less mature cells and resolve the appearing myelinlike sheets, MBP staining (green) was performed after cell permeabilization. Scale bar, $10 \mu \mathrm{m}$. 


\section{Stages of Myelin Sheet Expansion: "Spider Web", "Half-Moons", "Sheets"}

In cultures of developing oligodendrocytes three main stages of the membrane sheet expansion can be distinguished based on their morphology and antigen expression (see Figure 3.7).

Due to its appearance, we termed the most immature stage, which directly precedes expansion of the sheets, a "spider web" stage. The developing oligodendrocytes reach this stage by day 2 after shake. The cells at this stage extend a dense network of processes, positive for galactosylceramide (reactive with O1 or GalC antibodies), resembling spider webs. These processes have several orders of interconnecting branches. In common nomenclature cell at this stage can be referred to as immature oligodendrocytes and are described as "post-mitotic, non-migratory cells not yet elaborating myelin membranes" [317]. The "spider-web" oligodendrocytes are GalC-positive, but negative for such myelin-associated markers as MBP, surface PLP (see Figure 3.8, upper panel, 2 days) and MOG (data not shown).

In their most differentiated stage, the mature cultured oligodendrocytes are morphologically characterized by the formation of extensive myelin-like membrane sheets. In vivo and under co-culture conditions, these mature myelinating oligodendrocytes would wrap their myelin-like sheets around axons. Oligodendrocytes maintained in culture without contact to neurons spread their membrane extensions along the surface of the coverslip. Therefore, this developmental stage when oligodendrocytes have produced flat membrane sheets, which are not wrapped around axons, will be further referred to as the "sheets" stage. Mature oligodendrocytes show characteristic immunoreactivity for late myelin markers MBP, surface PLP (see Figure 3.8, lower panel, 6 days) and MOG. In accordance with literature, they keep immunoreactivity for the earlier myelin markers O1, O4, MAG, CNPase (GalC staining shown in the first image of the lower panel in Figure 3.8, MAG and CNPase stainings are in Figure 3.1).

With respect to myelin development, the transition between the "spider web" and the "sheets" stage is of particular interest. Here we have documented the stage during which oligodendrocytes undergo such transition (see Figure 3.7 and Figure 3.9). This transformation is very rapid, and can be observed within a narrow 


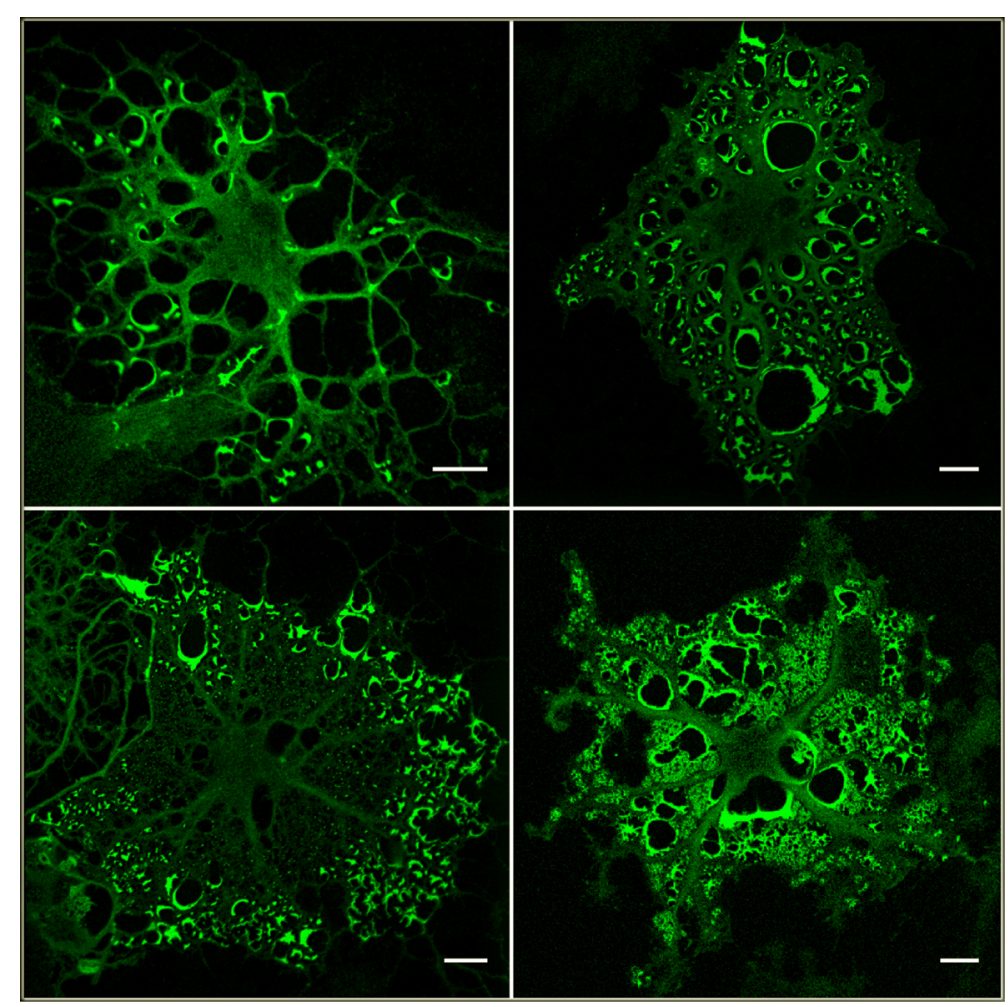

Figure 3.9: Cultured primary oligodendrocytes at a "half-moon" stage.

Mouse primary oligodendrocytes were cultured for 3 - 4 days after shake, fixed, permeabilized, immunostained for MBP (as described in Section 2.2.3) and subjected to confocal microscopy. Scale bar, $10 \mu \mathrm{m}$.

time-window (approx. 0.5 days, around day 3 after shake). At a "spider web" stage oligodendrocytes show very low intensity diffuse MBP staining in cytoplasm (see Figure 3.8, upper panel). In contrast, at the transition stage, a very bright and local MBP signal appears in between the processes of oligodendrocytes (shown in Figure 3.9). Due to the shape of this strong MBP staining, emerging at the branching points of oligodendrocyte processes, we termed this stage a "half-moon" stage.

During this stage, the gaps between intercepting and branching processes become smaller, as compared to the "spider web" stage. This could indicate that the gaps 

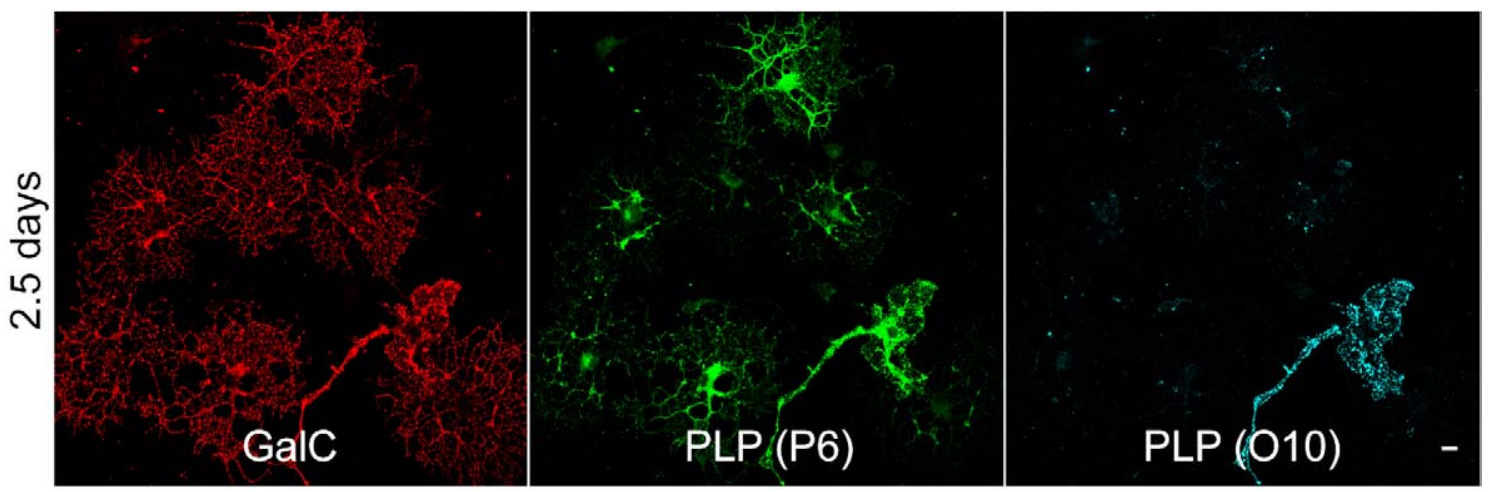

Figure 3.10: Total and surface PLP pools in developing oligodendrocytes.

After 2.5 days in culture, most pOLs are at the "spider-web" stage. At this time-point, some immature oligodendrocytes (GalC-positive cells, in red) already show vesicular accumulations of PLP in their cell bodies and processes (green). Total PLP pool, including intracellular vesicular PLP was visualized with P6 antibodies (green). After 2.5 days in culture, immunoreactivity for surface PLP (O10 antibody staining, cyan) is detectable only in few oligodendrocytes. Scale bar, $10 \mu \mathrm{m}$.

between the processes are being filled with MBP-positive membranes, in this way giving rise to myelin sheets.

\section{PLP Incorporation into the Plasma Membrane of Developing pOLs}

We chose endogenously expressed PLP as a marker protein to acquire the first insights in the process of new membrane incorporation into the growing myelin sheets. PLP is the most abundant myelin protein, comprising approx. $50 \%$ of the total protein amount in the CNS myelin. The plasma membrane incorporated pool of PLP can be resolved by live immunostaining of cultured oligodendrocytes with monoclonal IgM O10 antibodies, which specifically recognize the extracellular epitopes of PLP. On the other hand, the total PLP pool can be detected by immunostaining of fixed and permeabilized pOLs with rabbit polyclonal P6 antibodies.

At the immature "spider web" stage, pOLs start to exhibit immunoreactivity to P6 antibodies, which are specific for PLP. In these cells, the P6 signal is localized predominantly in the cell body. In comparison, no surface PLP signal could be 
registered in the cells at the "spider web" stage with O10 antibodies (see Figure 3.8, upper panel). Surface PLP signal is first detectable in some cultured pOLs after 2.5 - 3 days of development on coverslips, coinciding with the increase in numbers of P6-positive cells (see Figure 3.10). Thorough examination of the O10 stainings revealed that the initial PLP surface epitopes are localized in the processes of oligodendrocytes (see Figure 3.8, middle panel), whereas after 5 - 6 days on coverslips PLP is distributed over the MBP-positive membrane sheets (see Figure 3.8, lower panel).

Thus, plasma membrane incorporation of PLP (as judged from the O10 stainings) takes place around the same day, when dramatic increase in MBP expression can be detected (although MBP-positive, but O10-negative and vice versa cells were present in culture). Interestingly, at the "half-moon" stage PLP first appears in the regions of the oligodendroglial plasma membrane, where MBP is scarcely detectable.

\section{Changes in Actin Cytoskeleton}

Actin cytoskeleton is known to play important roles in cellular morphogenesis, membrane expansion, endo- and exocytosis. Since microfilaments could be involved in myelin membrane expansion, we analyzed distribution of F-actin at different stages of oligodendrocyte maturation. Dramatic rearrangements of actin cytoskeleton were observed with phalloidin stainings, as primary oligodendrocytes progressed in development. The cells at the "spider web" stage have profound actin filaments in their processes, but not at the locations where MBP appears (see Figure 3.11, upper panel). After 6 days in culture, filamentous actin was hardly detectable in mature oligodendrocytes, but was present in the non-oligodendroglial cells, serving as a control for phalloidin staining (see Figure 3.11, lower panel). MBP antibodies did not affect phalloidin binding, since down-regulation of microfilaments with maturation of oligodendrocytes was also observed in stainings performed with alternative myelin markers (data not shown).

In the analyzed developmental stages, between day 2 and day 5 in culture, tubulin cytoskeleton remained present in the major and minor processes of cultured oligodendrocytes (see tubulin staining in Figure 3.13). 


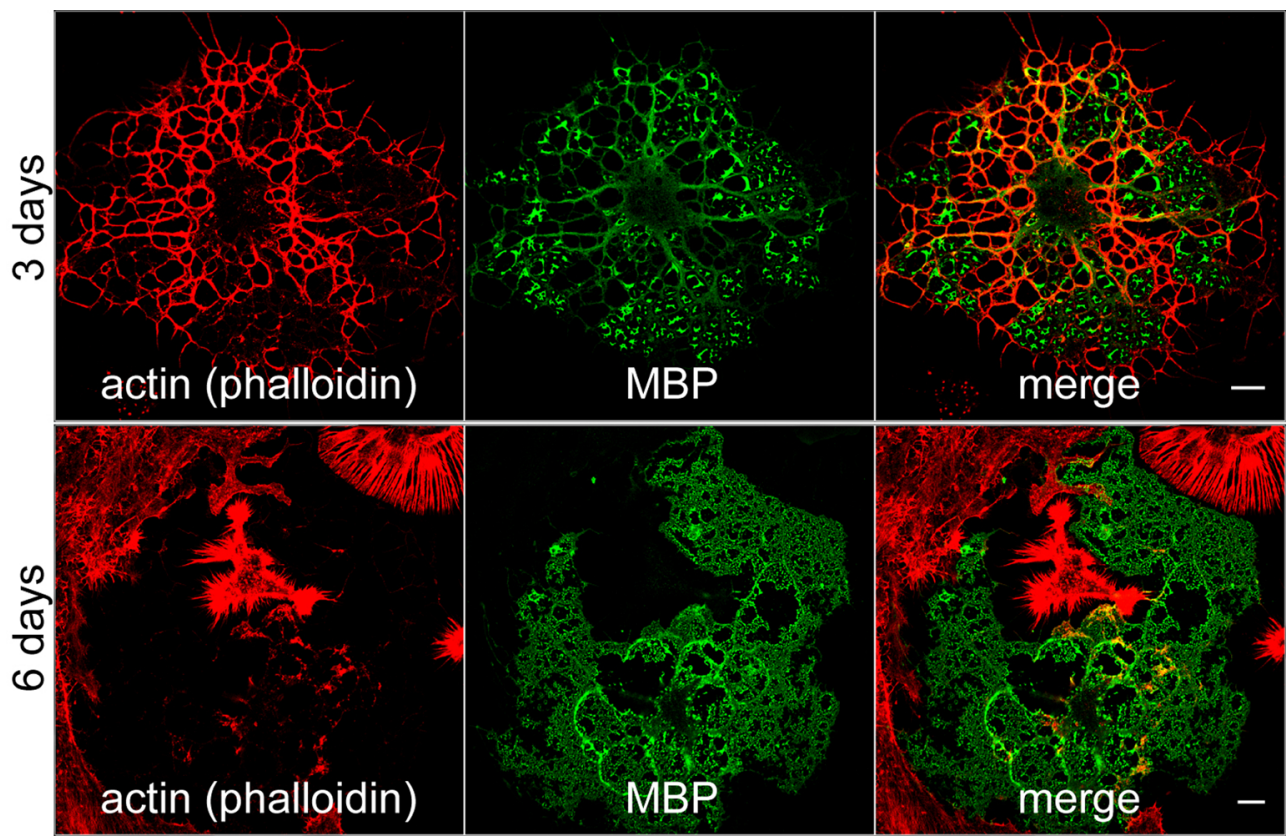

Figure 3.11: Microfilaments in developing and mature oligodendrocytes.

Oligodendrocytes were stained for filamentous actin (red) with Rhodamine-coupled phalloidin and with antibodies against MBP (green) to mark the myelin-like membrane sheets. The upper panel shows a less mature oligodendrocyte ( 3 days after shake), just starting to develop MBP-positive membrane domains. In the lower panel, a mature oligodendrocyte (possessing extended MBP-positive sheets), surrounded by non-oligodendroglial cells is depicted. In immature oligodendrocytes microfilaments are clearly stained, whereas mature oligodendrocytes are almost deficient of filamentous actin. Scale bar, $10 \mu \mathrm{m}$.

\subsubsection{Distribution of Membrane Extension Players in pOLs}

Plasma membrane expansion is fine-tuned by regulating the balance between incorporation of the newly synthesised or recycled membrane (exocytosis) and internalization of the cell surface membrane (endocytosis). This Section will focus on analysis of the first component of membrane extension, i.e. new membrane biosynthesis, its trafficking to the plasma membrane and incorporation into the growing membrane sheet by exocytosis. Endocytosis has been previously shown to be down-regulated during the active phase of myelin growth [201]. Further characterization of endo- 
cytosis in developing oligodendrocytes is documented in Appendix C (Figure C.1, Figure C.2, Figure C.3).

\section{Localization of Biosynthetic Machinery}

De novo production of plasma membrane components is carried out by the cellular biosynthetic machinery. To analyze subcellular distribution of the components of the biosynthetic machinery in mouse primary oligodendrocytes, immunocytochemistry in conjunction with confocal microscopy were employed. To label endoplasmic reticulum (ER), immunostaining for ER chaperone calnexin was performed; cisGolgi apparatus was detected with antibodies against residual Golgi marker protein GM130; trans-Golgi network was highlighted with antibodies against $\gamma$-adaptin; ribosomes were detected with antibodies against phosphorylated form of ribosomal protein S6 (PS6). Localization of these markers was analyzed with respect to MBP or O1 stainings, visualizing the myelin-like membrane sheets of oligodendrocytes.

Ribosomes (PS6 staining) were highly enriched in the cell bodies and processes of oligodendrocytes, but were excluded from MBP-positive cellular domains. This result is especially surprising, since MBP is known to be transported in the form of mRNA followed by local translation [191], which implies close proximity of MBP and ribosome epitopes. Nevertheless, MBP and PS6 signals in co-stained primary oligodendrocytes demonstrate almost mutually exclusive localization (see Figure 3.12, upper panel).

ER was predominantly localized in the cell body and processes of oligodendrocytes, cis-Golgi showed perinuclear localization in the cell body, whereas trans-Golgi staining could also be detected in the processes of pOLs (see Figure 3.12). No components of the biosynthetic machinery were enriched in the myelin-like MBP-positive membrane sheets of oligodendrocytes.

\section{Localization of Vesicular Trafficking Machinery}

Cytoskeleton is indispensable for vesicular trafficking in the cells. Tubulin staining of pOLs demonstrated, that microtubules are localized in the cell body, main and minor oligodendrocyte processes, but are excluded from the MBP-positive sheets 

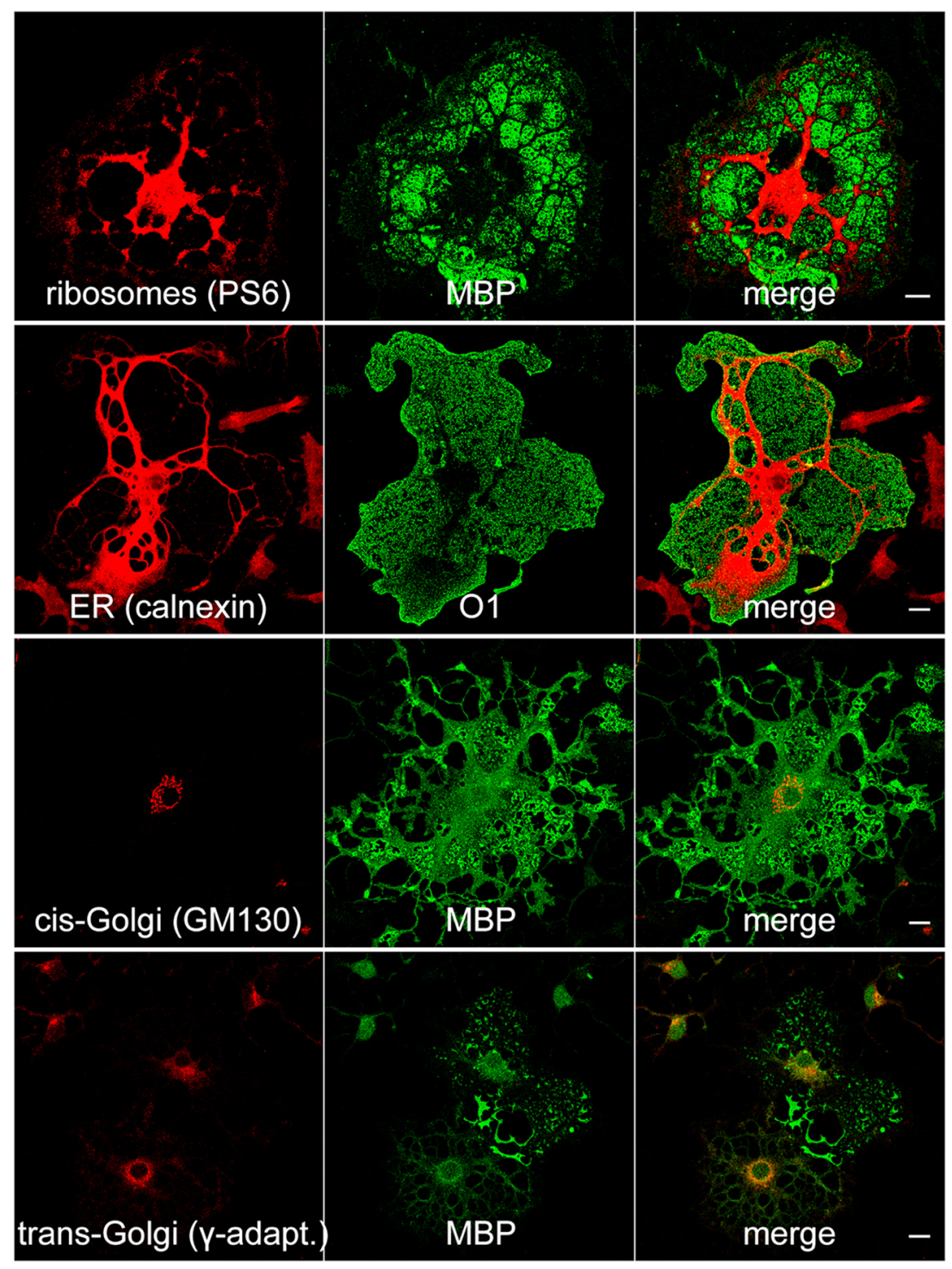

Figure 3.12: Subcellular distribution of the biosynthetic machinery in primary oligodendrocytes.

To detect components of the biosynthetic machinery, antibodies against the following epitopes were used (left column, all signals in red): PS6 for ribosomes, calnexin for ER, GM130 for cis-Golgi and $\gamma$-adaptin for trans-Golgi network. Myelin-like sheets of oligodendrocytes are stained with either anti-MBP or $\mathrm{O} 1$ antibodies (central column, in green). Scale bar, $10 \mu \mathrm{m}$. 


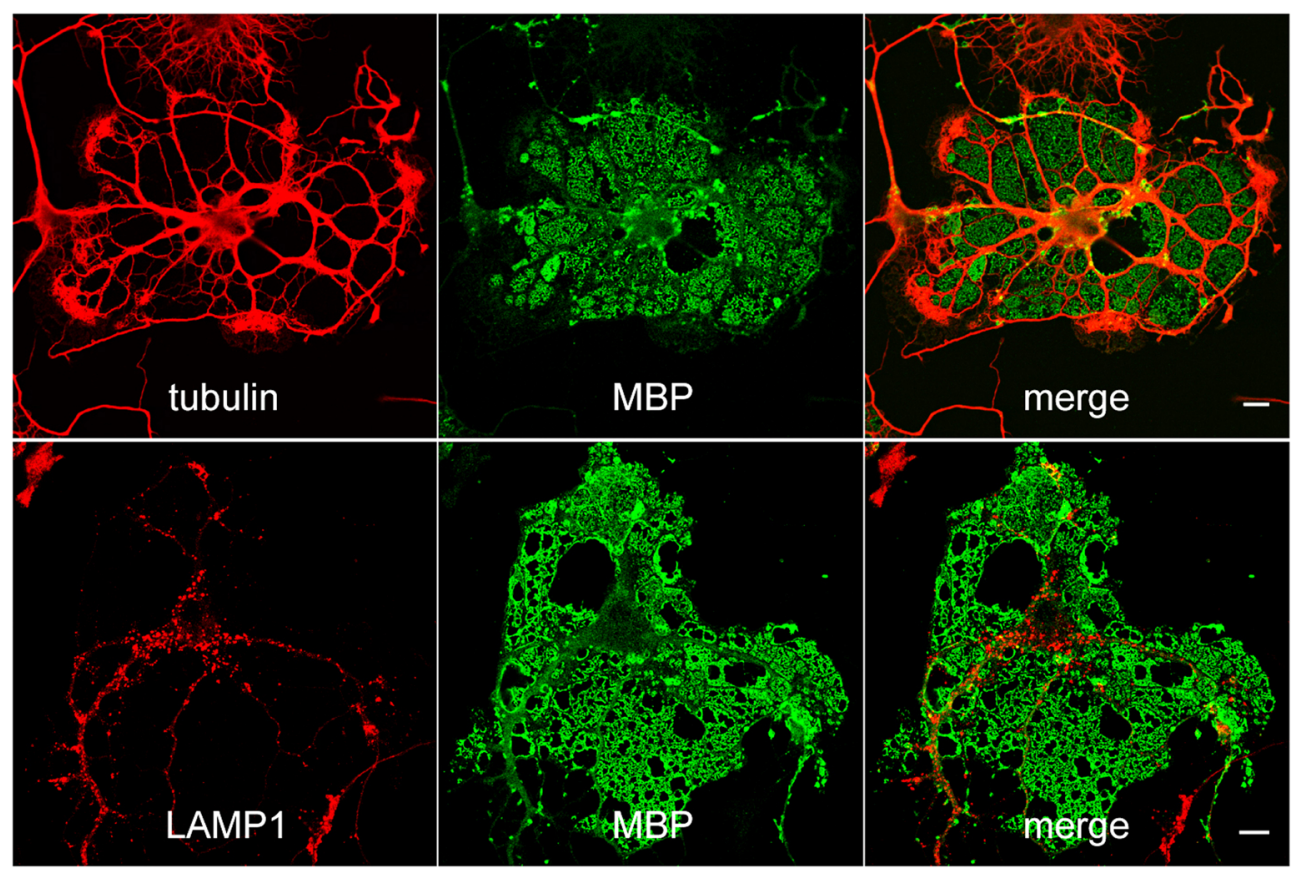

Figure 3.13: Subcellular distribution of microtubules and endosomes in pOLs.

Cultured primary oligodendrocytes were co-stained for tubulin (red) and MBP (green), as shown in the upper panel, or for late endosomal/lysosomal marker LAMP1 (red) and MBP (green), as depicted in the lower panel. Merge images demonstrate, that neither microtubules, nor late endosomes/lysosomes can be found in the MBP-positive membrane sheets. Scale bar, $10 \mu \mathrm{m}$.

(see Figure 3.13). Actin cytoskelton is also localized to the cell body and processes in less mature oligodendrocytes ("half-moon" stage), but is almost depleted from the mature pOLs (see Figure 3.11). To characterise subcellular distribution of the endosomal intermediates of vesicular trafficking, pOLs were stained with the antibodies against LAMP1, a late endosomal/lysosomal marker. Late endosomes/lysosomes were localized to the cell bodies and processes of oligodendrocytes, including the outermost rim of the cells. MBP-enriched regions of pOLs did not contain LAMP1positive vesicles (see Figure 3.13). 


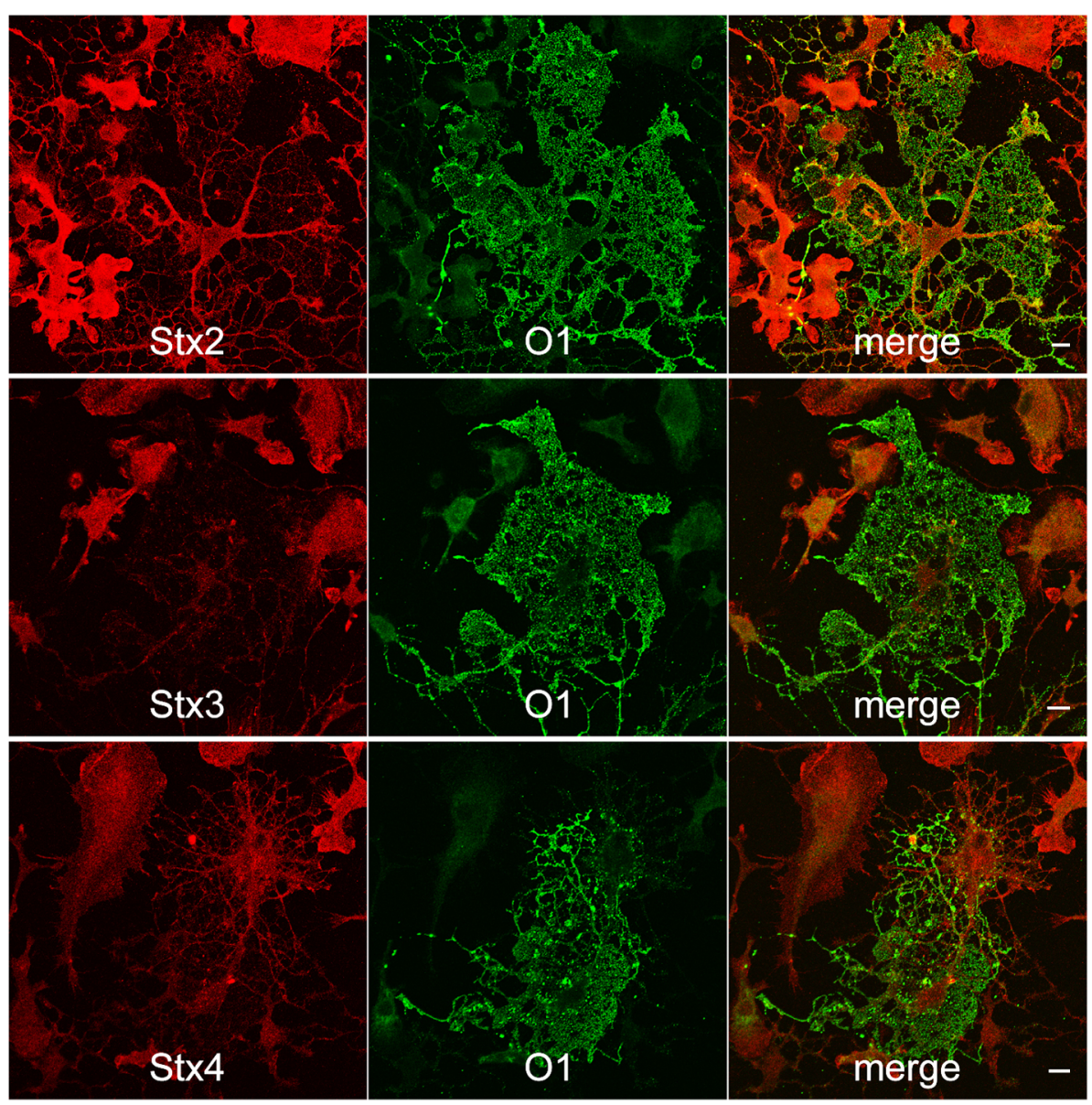

Figure 3.14: Localization of SNARE proteins syntaxins 2 - 4 in pOLs.

Plasma membrane Q-SNAREs syntaxin 2, 3, 4 (left column, red) were detected with corresponding antibodies in cultured pOLs. In these cultures, oligodendrocytes were discriminated from other cell types with staining for oligodenroglial plasma membrane marker $\mathrm{O} 1$ (green). In pOLs, confocal microscopy revealed weak diffuse signals of syntaxins, which were enriched in cell bodies and processes of oligodendrocytes. No preferential sites for syntaxins could be detected in the sheets. Scale bar, $10 \mu \mathrm{m}$.

\section{Localization of Vesicular Fusion Regulators}

Newly synthesised membrane is incorporated into the existing plasma membrane sheet by exocytosis. To determine whether oligodendrocytes have preferential myelin 


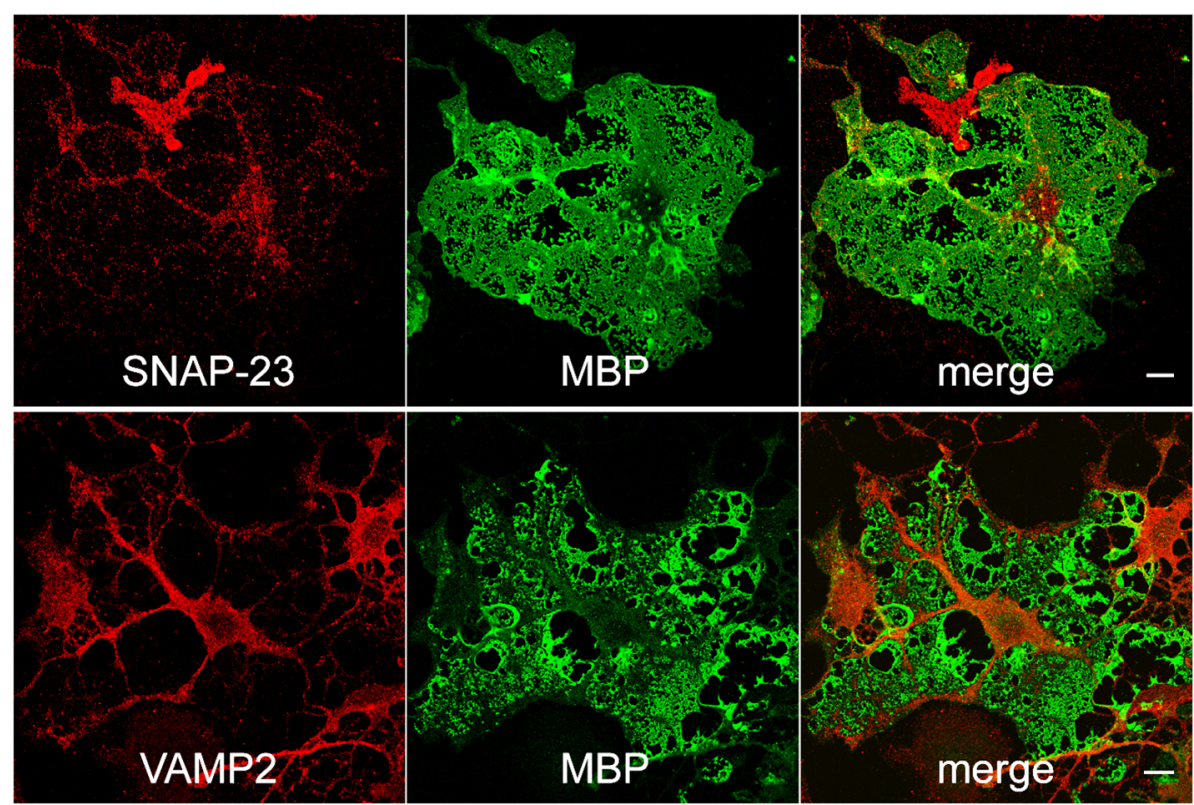

Figure 3.15: Localization of SNARE proteins SNAP-23 and VAMP2 in pOLs.

Immunoreactivity for Q-SNARE protein SNAP-23 and vesicular R-SNARE protein VAMP2/synaptobrevin is shown in red, MBP immunostaining is in green. Neither SNAP23, nor VAMP2 were enriched in MBP-positive sheets. Scale bar, $10 \mu \mathrm{m}$.

membrane expansion sites ("hot-spots" of exocytosis), we analyzed subcellular distribution of mediators and regulators of exocytosis.

Vesicle fusion is mediated by SNAREs (soluble N-ethylmaleimide sensitive factor attachment protein receptors) [139]. Immunostainings of pOLs for SNAREs, which are implicated in vesicular fusion to plasma membrane revealed their preferential localization in the processes of oligodendrocytes (stainings for syntaxins are documented in Figure 3.14, immunoreactivity of SNAP-23 and VAMP2 is shown in Figure 3.15).

Rab GTPases contribute to the specificity of vesicular docking, a step preceding vesicular fusion [146]. Rab3 proteins, which are the most likely exocytic Rab proteins in pOLs, were enriched in the cell bodies and processes of pOLs (see Figure 3.16).

The exocyst component Sec8, which is implicated in oligodendrocyte differentiation [180], also showed predominant localization in the processes and cell bodies 


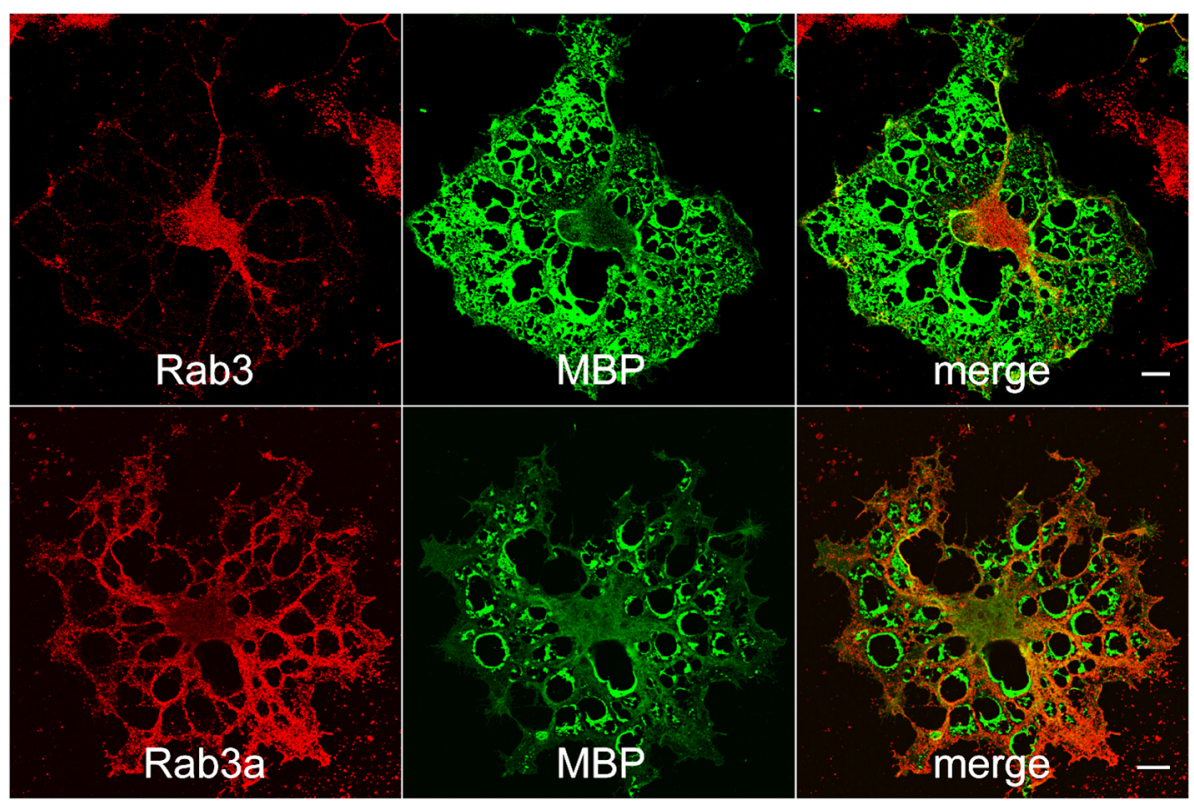

Figure 3.16: Localization of Rab3 and Rab3a in pOLs.

Co-staining of primary oligodendrocytes using antibodies against Rab3 (red, upper panel) and Rab3a (red, lower panel) in conjunction with antibodies against MBP (green). Rab3 proteins do not show enrichment in MBP-positive membrane sheets. Scale bar, $10 \mu \mathrm{m}$.

of oligodendrocytes, as well as the phosphatidylinositol 4,5-bisphosphate (PIP2), which plays regulatory functions both in endo- and exocytosis (see Figure 3.17). Previously, interaction of PIP2 and MBP has been reported [318]. This suggests that in immunostainings MBP might mask some of the PIP2 epitopes from the antibodies. To rule out this possibility, PIP2 staining can be verified in MBP-deficient Shiverer oligodendrocytes.

The proteins clathrin and caveolin, which are important for membrane turnover, were also enriched in the cell bodies and processes of oligodendrocytes Figure 3.18.

Taken together, the components necessary for myelin membrane extension are enriched in the processes and cell bodies of oligodendrocytes, but almost excluded from the MBP-positive membrane sheets. 


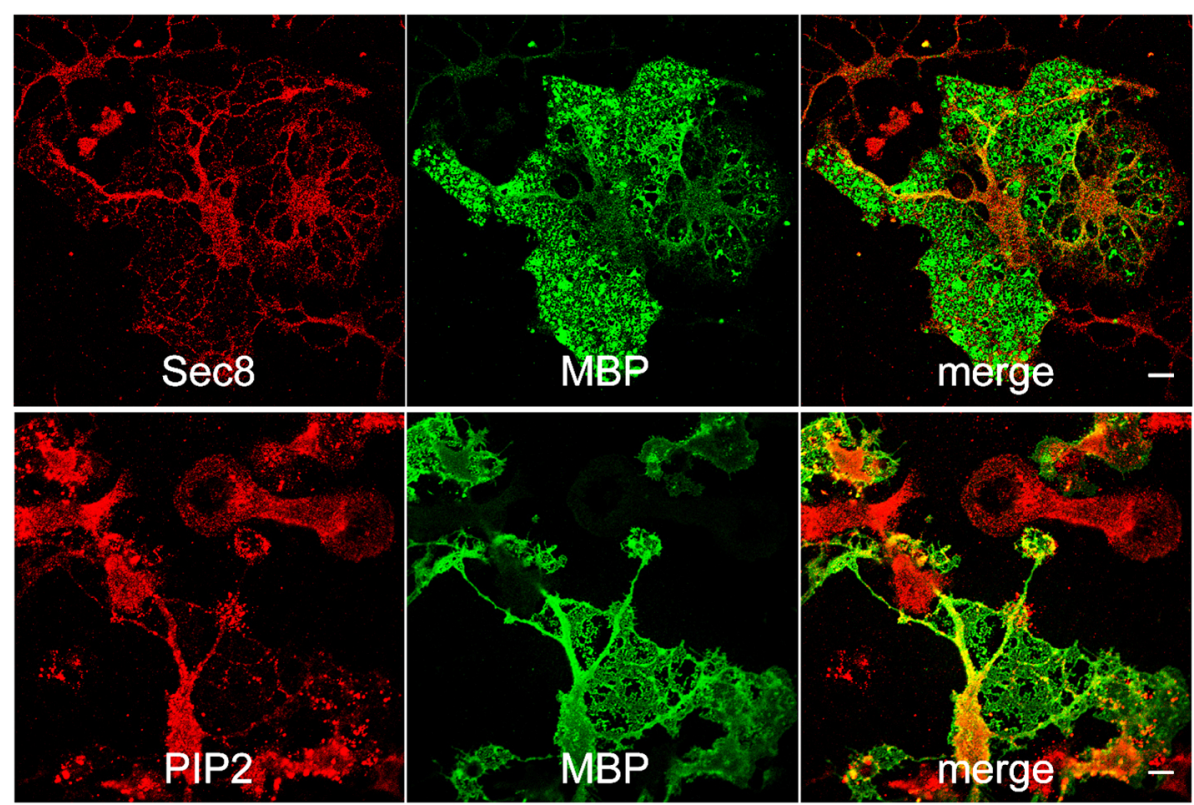

Figure 3.17: Localization of exocyst and signaling lipid PIP2 in pOLs.

Antibodies detecting Sec8 exocyst component (red) and antibodies directed against lipid phosphoinositide $\mathrm{PI}(4,5) \mathrm{P} 2$ (PIP2) (red) were used in co-stainings of oligodendrocytes for MBP (green). Sec8 and PIP2 signals were enriched in cell bodies and processes of oligodendrocytes, but not in the MBP-positive membrane sheets. Scale bar, $10 \mu \mathrm{m}$.

\subsubsection{Where does Vesicular Trafficking Take Place in pOLs?}

Analysis of distribution of biosynthetic and vesicular trafficking and fusion machineries in pOLs indicated their preferential localization to the cell bodies and processes of oligodendrocytes. In order to support the hypothesis that intracellular trafficking of myelin proteins takes place in the aforementioned domains, we examined localization of the transport pools of compact myelin proteins.

In order to distinguish the intracellular and surface protein pools, a mammalian expression construct was designed and generated, which allowed overexpression of recombinant compact myelin protein MOG. Two small peptide tags myc and HA, separated by a thrombin cleavage site were fused to the extracellular N-terminus of MOG (as depicted in scheme (a), Figure 3.19, for the primary sequences of the tags and for the DNA sequence of the construct refer to Appendix A). 


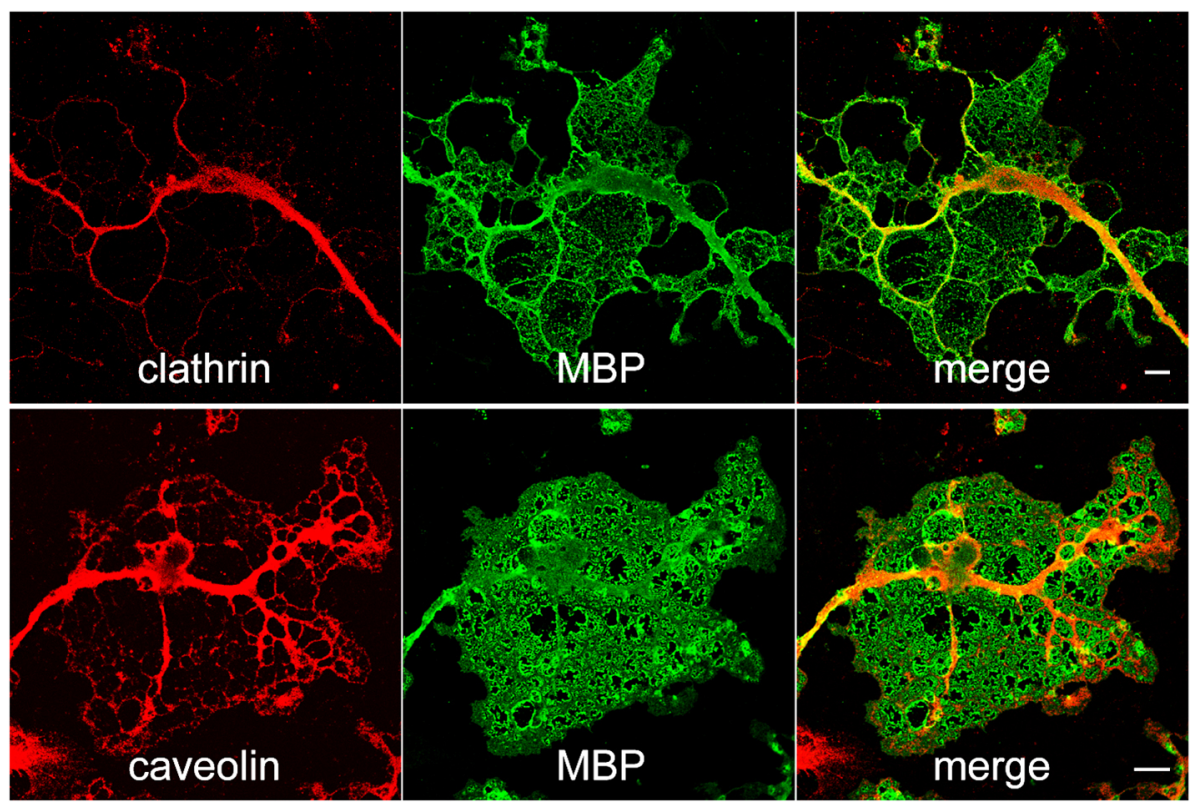

Figure 3.18: Localization of clathrin and caveolin in pOLs.

Primary oligodendrocytes were co-stained for clathrin (red) and MBP (green) or for caveolin (red) and MBP (green). Merge images show no enrichment of neither clathrin, nor caveolin signals in MBP-positive membrane sheets. Scale bar, $10 \mu \mathrm{m}$.

Correct plasma membrane localization of the overexpressed MOG was confirmed by analyzing its colocalization with the endogenous MBP in transiently transfected pOLs (Lipofectamine transfection was performed as described in Section 2.2.3). For this, Pearson's correlation coefficient values between exogenously expressed MOG (myc-thr-HA-MOG) and MBP were calculated and were shown not to differ significantly from the values obtained for the endogenous MOG, as displayed on the graph (b), Figure 3.19.

After incubation with thrombin (40 $\mathrm{min}, 0.02 \mathrm{U} / \mu \mathrm{L}$ of thrombin protease (Novagen/Merck KGaA, Darmstadt, Germany) in modified Krebs-Ringer solution, Section 2.2.4), the myc-tag was cleaved off the plasma membrane of transfected pOLs. Since thrombin cannot enter the cells, the remaining myc epitopes marked exclusively the intracellular MOG. Surface staining for HA tag was performed to resolve the plasma membrane incorporated MOG pool. Permeabilization of the cells fol- 


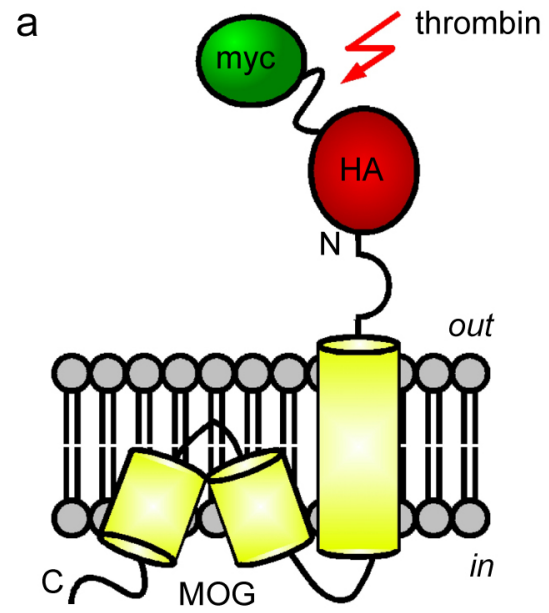

b Correlation of endogenous MOG and overexpression MOG construct with MBP

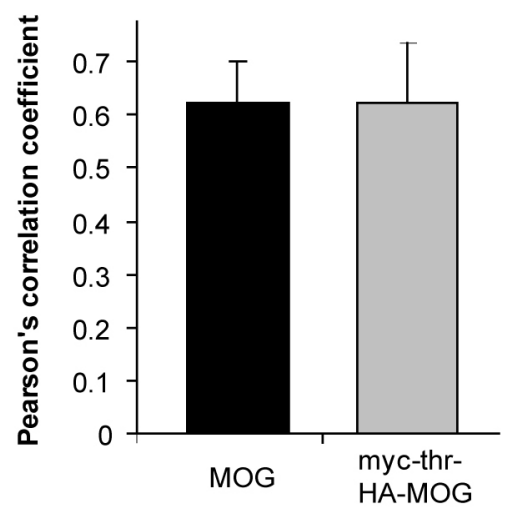

C

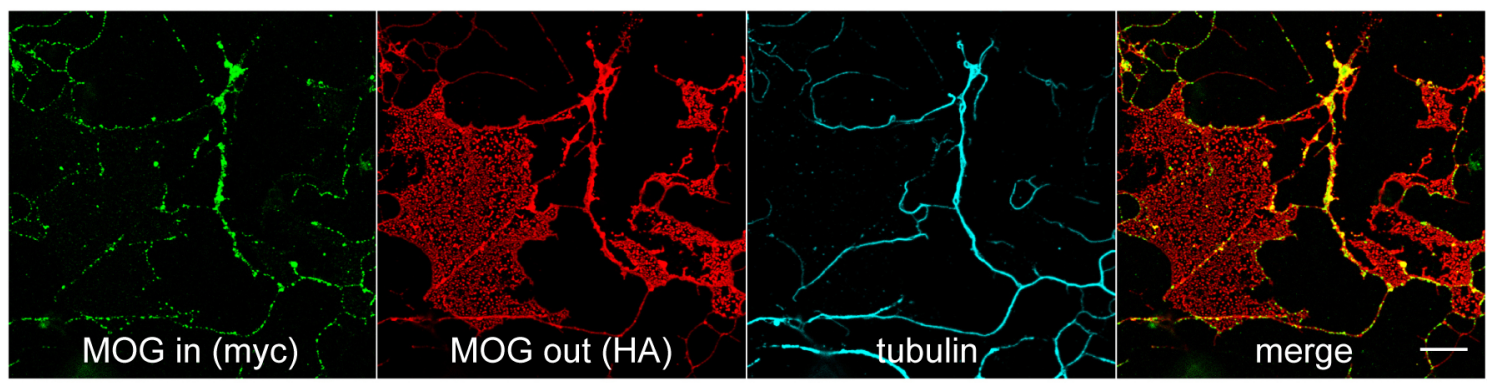

Figure 3.19: Discrimination of intracellular and surface pools of myelin protein MOG.

(a) Schematic representation of myc-thr-HA-MOG overexpression construct. Extracellular domain of a transmembrane protein MOG carries myc and HA tags, separated by a thrombin cleavage site. (b) Comparison of Pearson's correlation coefficient values between exogenously expressed MOG (myc-thr-HA-MOG) and MBP with the correlation values between endogenous MOG and MBP. Mean values \pm SD are plotted, $n=19-20$. Colocalization of myc-thr-HA-MOG with MBP did not differ significantly from colocalization of endogenous MOG with MBP, confirming correct plasma membrane targeting of the overexpression construct. (c) To resolve plasma membrane incorporated and transport pools of MOG, thrombin cleavage of the surface myc tag was carried out, followed by detection of the plasma membrane MOG pool by surface staining against HA tag (red), labeling of intracellular MOG pool with anti-myc antibodies after permeabilization of the cells (green) and co-staining with antibodies against tubulin (cyan) to mark the processes of oligodendrocytes. Scale bar, $10 \mu \mathrm{m}$. 
lowed by immunostaining for myc epitopes allowed detection of the intracellular MOG pool. Confocal microscopy demonstrated that, whereas plasma membrane incorporated MOG was distributed over the myelin-like membrane sheets, the internal MOG pool was restricted to the processes and cell bodies of oligodendrocytes (see Figure 3.19).

\subsubsection{Thickness of Myelin Membrane Sheets}

In primary oligodendrocytes vesicular trafficking regulators and transport intermediates were detected predominantly in the processes and cell bodies, as shown in previous chapters. In contrast to that, myelin-like sheets of pOLs seemed to be depleted of the components of vesicular trafficking machinery, although they should require vesicular trafficking for expansion.

To assess which subcellular domain of cultured primary oligodendrocytes is permissive for vesicular trafficking, their thickness was measured with atomic force microscopy (AFM). The height of the processes of pOLs was reaching $150-200 \mathrm{~nm}$, which is permissive for vesicular trafficking. The height of the MBP-positive regions was $43.99 \pm 8.43 \mathrm{~nm}$ (mean $\pm \mathrm{SD}$ from 6 independent experiments), which corresponds to approx. $35 \mathrm{~nm}$ thickness of the cytosolic layer in between the plasma membranes, that might not be sufficient to allow extensive vesicular trafficking.

Closer apposition of the MBP-positive plasma membranes, which we observed with AFM in pOLs, is supported by our EM data that shows compaction of the MBP-positive membranes in pOLs (see Figure 3.6).

\subsubsection{Mapping Fusion Sites on pOLs Plasma Membrane}

To identify where the new membrane is added to the extending plasma membrane of pOLs, total internal reflection (TIRF) microscopy was employed. For TIRF microscopic analysis, fusion constructs for overexpression of compact and non-compact myelin proteins, tagged with pH-sensitive GFP (pHluorin) were designed and generated (see Appendix A). Transfection of pOLs with pHluorin-MAG, pHluorin-MOG or pHluorin-TM4PLP constructs allows visualization of green fluorescent signals from corresponding proteins on the plasma membrane, masking intracellular fluores- 


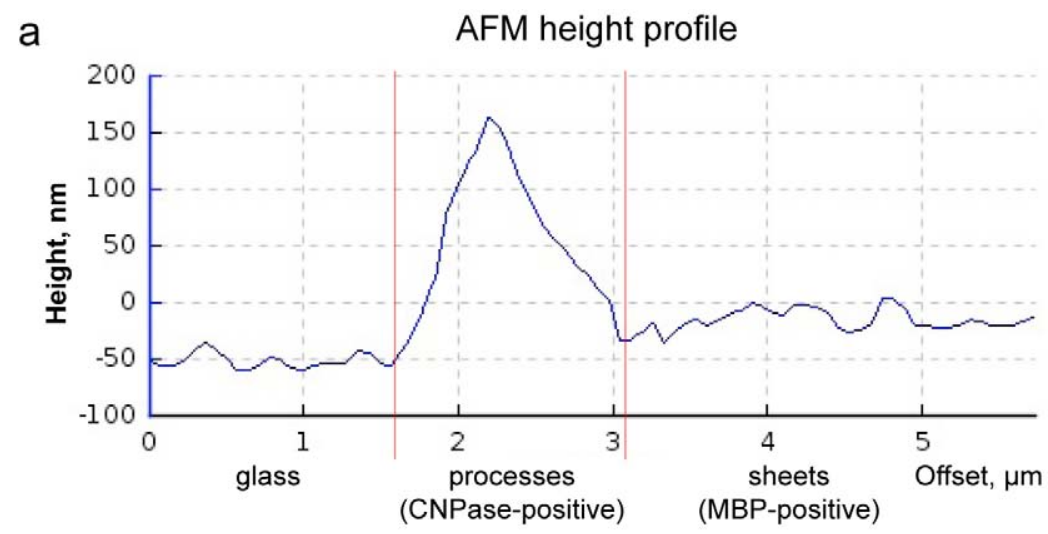

b
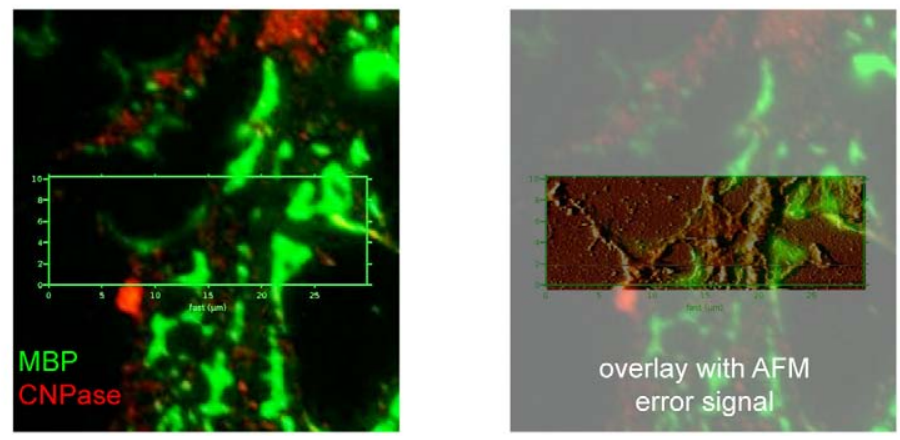

Figure 3.20: Thickness of myelin-like membrane sheets measured with AFM in pOLs.

(a) A representative AFM height profile along a $5.7 \mu \mathrm{m}$ line from one measured oligodendrocyte is shown. The height of the measured oligodendroglial process (CNPasepositive area) reaches $200 \mathrm{~nm}$, whereas the height of the myelin-like membrane sheet (MBP-positive) is only $25-50 \mathrm{~nm}$.

(b) Fluorescent image of the measured area of a primary oligodendrocyte displays immunolabeling for CNPase (red) and MBP (green). Overlay of a fluorescent image with an AFM error image (calculated from deflection of cantilever) reflects height of the depicted area of oligodendrocyte.

cence due to lower $\mathrm{pH}$ in endosomal transport intermediates. With these constructs we sought to detect the sites of plasma membrane incorporation of tagged proteins. Precise mapping of sites of exocytosis in pOLs involves live labeling of processes and sheets with additional markers and requires further experiments. The results acquired so far indicate that vesicles carrying compact and non-compact myelin proteins have similar fusion preferences to the processes domains of pOLs. 
a

FRAP antiGalC-FabRhodamine

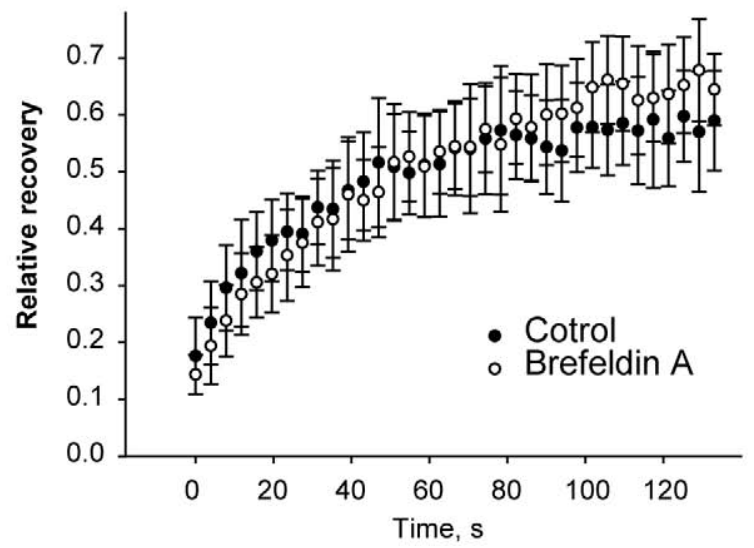

b

FRAP antiGalC-FabRhodamine

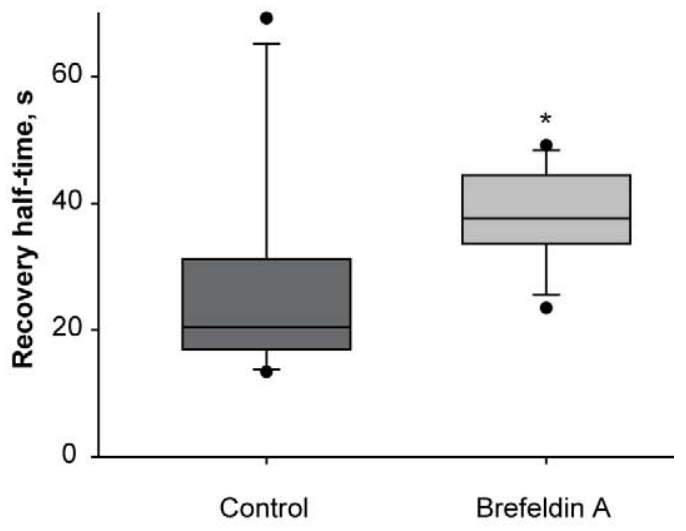

Figure 3.21: Brefeldin A treatment slows down lateral diffusion of GalC on pOLs plasma membrane.

(a) Relative fluorescence intensity recovery curves for control and brefeldin A treated cells are plotted as a function of time after bleach. Data represents means $\pm S D, n=10-11$.

(b) Recovery half-time is significantly shorter for control cells, than for brefeldin $A$ treated cells. The groups were compared with the Mann-Whitney Rank Sum Test; * $P<0.05, n=$ $10-11$.

\subsubsection{Lateral Diffusion on Plasma Membrane of pOLs}

The data outlined in previous Sections suggest that membrane biosynthesis, trafficking and possibly plasma membrane incorporation take place in the processes and cell bodies of oligodendrocytes, but not in the myelin-like sheets. Therefore, the myelin-like membrane sheets could be produced by lateral flow of the plasma membrane, which is driven by incorporation of new membrane into the processes of pOLs.

To verify whether a flow component contributes to the mobility of molecules on the plasma membrane of pOLs, the primary cultures were treated with brefeldin A $(10 \mu \mathrm{g} / \mathrm{mL}$, pre-incubation $1 \mathrm{~h})$. Brefeldin A induces disassembly of Golgi apparatus and within $40 \mathrm{~min}$ depletes secretory trafficking to the plasma membrane [319]. The rate of lateral diffusion on the plasma membrane of pOLs was assessed with fluorescence recovery after photobleaching (FRAP) approach. Plasma membrane 
a

FRAP Cell Mask Orange

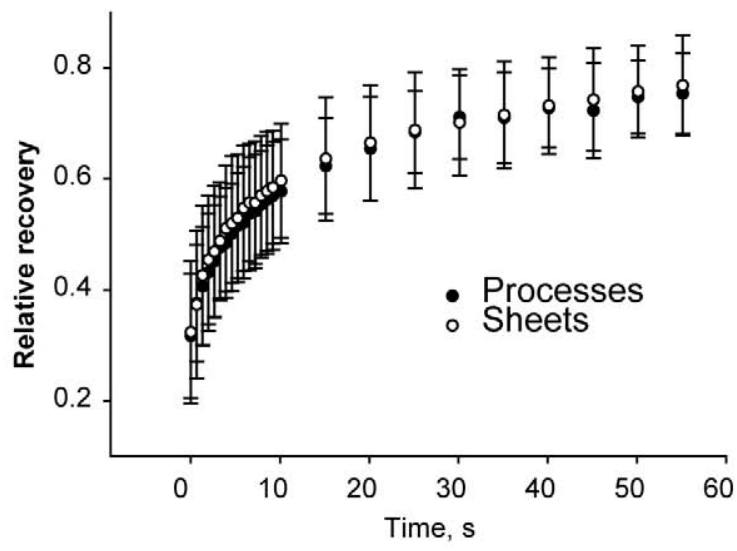

C

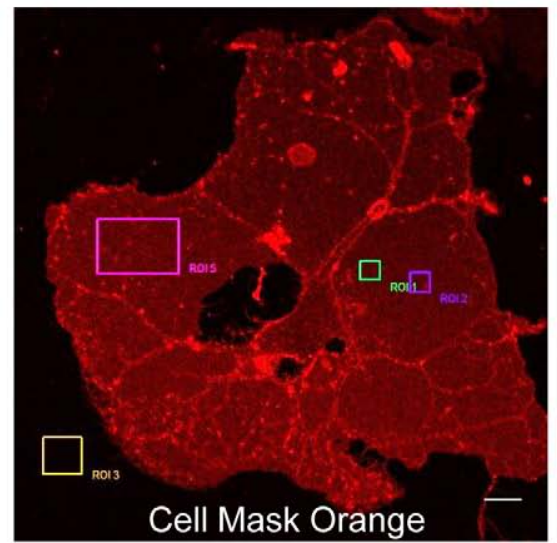

b FRAP Cell Mask Orange

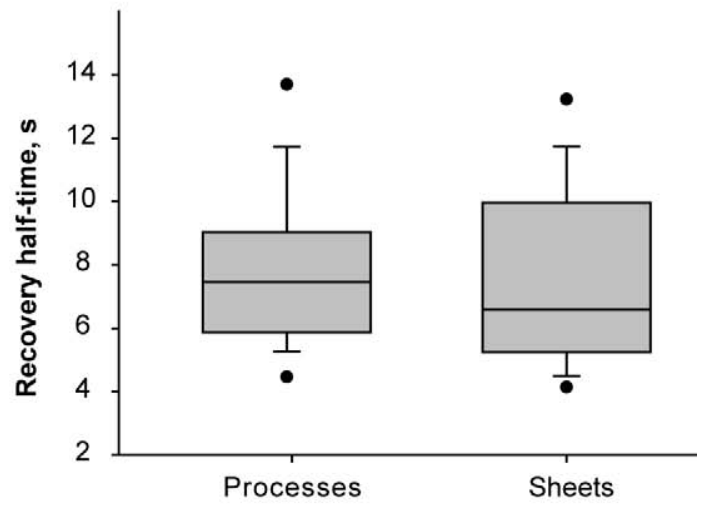

Figure 3.22: FRAP rates in myelin-like membrane sheets proximally and distally to the processes.

(a) Relative fluorescence intensity recovery curves of the Cell Mask Orange lipid dye in the myelin-like membrane sheets proximally to the processes (here labelled as "Processes") and distally from the processes (here labelled as "Sheets") are plotted. Data represents means $\pm S D, n=17$.

(b) No significant difference was detected in recovery half-time of the Cell Mask Orange lipid dye in the myelin-like membrane sheets proximally to the processes and distally from the processes, $\mathrm{n}=17$.

(c) A typical Cell Mask Orange staining of a primary oligodendrocyte is shown, with ROI1 and ROI2 corresponding to the bleached regions, located proximally to the processes and distally from the processes, respectively. Scale bar, $10 \mu \mathrm{m}$. 
galactosylceramides in pOLs were live immunolabelled with mouse monoclonal IgG primary antibodies directed against GalC, and secondary anti-IgG Fab fragments, coupled to fluorophore Rhodamine. Live imaging was performed in modified KrebsRinger solution (see Section 2.2.4); photobleaching of $5 \times 5 \mu \mathrm{m}$ plasma membrane areas was achieved in 4 scans with full power 561 laser; fluorescence recovery was recorded for 2 min or until it reached plateau, as described in Section 2.2.4. Cells incubated during imaging with brefeldin A had significantly longer recovery halftime, indicating reduced mobility rates of GalC lipids on the surface of pOLs, when the secretory pathway is blocked.

These results suggesting that activity of the biosynthetic-secretory pathway contributes to the diffusion rates on pOLs plasma membrane were reproduced by $\mathrm{S}$. Aggarwal (AG Simons) using a lipid dye Cell Mask Orange instead of antibodies for GalC.

To acquire insights in the directionality of diffusion, FRAP values were compared in the plasma membrane areas, located proximally to the main processes and distally from the processes, e.g. in the center of the membrane sheets. Primary oligodendrocytes were stained with a lipid dye Cell Mask Orange; bleaching of two $5 \times 5 \mu \mathrm{m}$ plasma membrane areas and recording of recovery curves were performed simultaneously. No significant difference in recovery rates between the proximal and distal to the major processes areas was detected, possibly due to the limitation of the staining, which does not allow to resolve the minor oligodendroglial processes.

To further clarify the directionality of the diffusion in the plasma membrane sheets of primary oligodendrocytes, experiments involving single particle trafficking (SPT) or fluorescence correlation spectroscopy (FCS) can be helpful.

\subsection{Molecular Mechanisms of Myelin Membrane Orga- nization}

The CNS myelin is formed by the myelin-producing glial cells, oligodendrocytes. During the myelination process, myelin membrane is synthesised in vast amounts in these cells and delivered to the cell surface, where it is incorporated into a growing 
myelin sheet. The myelin membrane is morphologically and functionally distinct from the plasma membrane of the oligodendroglial cell body. Furthermore, the myelin membrane itself is not homogeneous either. In developed myelin, one can distinguish compact and non-compact areas, which contain different amounts of cytosol and are characterized by distinct membrane composition. The molecular mechanisms underlying this membrane domain formation are not well understood. One possibility is that these membranes are pre-assembled into functional domains in the biosynthetic pathway and then targeted specifically to the corresponding cell surface domain. However, our data on intracellular trafficking in oligodendrocytes indicates that the bulk myelin membrane (destined for both compact and noncompact myelin) is first incorporated into the processes and cell bodies of growing oligodendrocytes (described in previous Section 3.2). This raises the next question: how does the plasma membrane of oligodendrocytes further segregate into distinct domains?

\subsubsection{Properties of Membrane Domains in pOLs}

\section{Morphological and Biochemical Visualization of Membrane Domains in pOLs}

In culture, mouse primary oligodendrocytes (pOLs) develop flat membrane extensions, which resemble myelin membrane, generated by oligodendrocytes in vivo. Plasma membrane domains in cultured primary oligodendrocytes can be distinguished both morphologically and biochemically (as described in Section 3.1). With respect to morphology, the processes and cell bodies of pOLs are enriched in noncompact myelin proteins MAG and CNPase, contain tubulin cytoskeleton and carry characteristics of non-compact myelin membrane, whereas the sheets are enriched in compact myelin protein MBP (see Figure 3.23), similarly to the membrane of the compact myelin in vivo. As profile plots demonstrate, the maxima gray values of MBP and MAG signals do not coincide (see Figure 3.23, (c)). In contrast to MBP intensity profile, the profile of tubulin signal shows maxima in the same positions where MAG intensity peaks are located (see Figure 3.23, (d)). 

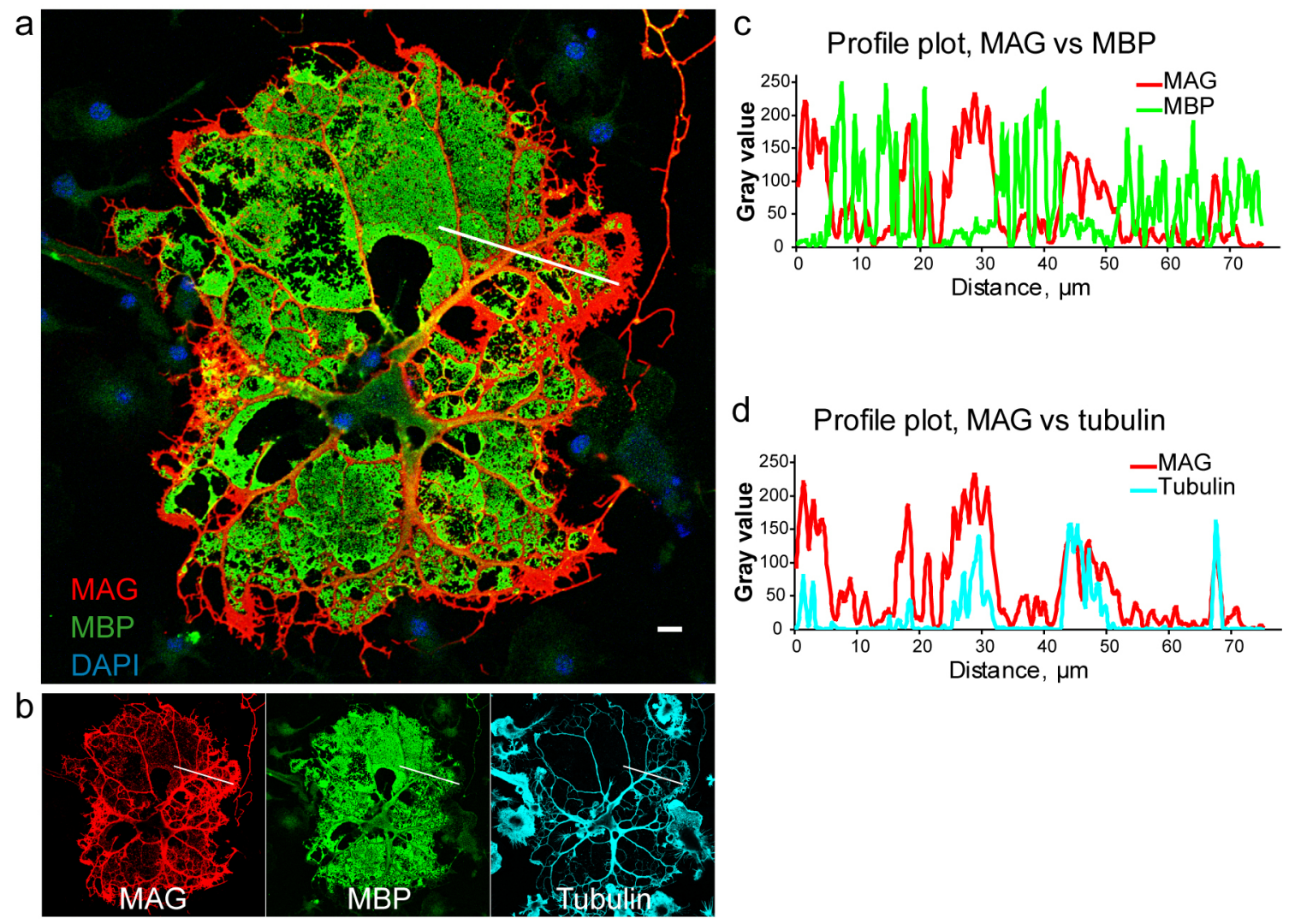

d Profile plot, MAG vs tubulin

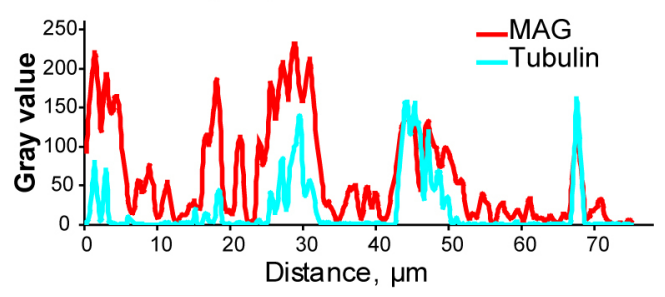

Figure 3.23: Visualization of distinct plasma membrane domains in pOLs with confocal microscopy.

Primary mouse oligodendrocytes were co-immunostained for endogenous myelin proteins MBP (green) and MAG (red), and for tubulin cytoskeleton (cyan). Nuclei were visualized with DAPI staining (blue). Scale bar, $10 \mu \mathrm{m}$.

(a) An overlay image of MBP (green), MAG (red) and DAPI (blue) signals is shown. Note segregation of red and green signals, reflecting distinct localization of non-compact myelin protein MAG and compact myelin protein MBP on pOLs plasma membrane.

(b) Imaged channels, corresponding to MAG, MBP and tubulin are shown separately. (c and d) To compare the distribution of MBP, MAG and microtubules in primary oligodendrocytes, intensity profiles of MAG, MBP and tubulin fluorescent signals were plotted along an approx. $80 \mu \mathrm{m}$ line (white line, denoted on the fluorescence images).

Distinct plasma membrane domains of pOLs can also be separated biochemically, as described in the first part of the Results Chapter. In sucrose gradients, compact 
myelin transmembrane proteins PLP and MOG reside in fractions of all densities, whereas the non-compact transmembrane myelin protein MAG was restricted to the heavier fraction (see Figure 3.5).

We have further characterized physiological properties of the plasma membrane domains in pOLs. With endocytosis assays we demonstrated that the non-compact myelin protein MAG undergoes fast turnover on pOLs plasma membrane. In contrast, the compact myelin components, such as PLP, MOG and GalC are endocytosed significantly slower (see images and quantifications in Appendix C, Figure C.2 and Figure C.3). In overexpression studies we observed that the fluorescent signals of the soluble cytosolic proteins GFP and RFP were hardly detectable in the MBPpositive sheets, but were clearly marking the cell bodies and processes of transfected oligodendrocytes (see Figure C.5 in Appendix C). Interestingly, the transmembrane proteins, tagged with GFP on the intracellular domain also showed preferential localization to the plasma membrane of the cell bodies and processes of oligodendrocytes. These proteins were restricted from entering the MBP-positive plasma membrane sheets (see Figure C.4 in Appendix C).

As illustrated in Figure 3.23, development of the compact-myelin-like and the non-compact-myelin-like membrane domains in pOLs does not require contact with neurons. Therefore, cultured primary oligodendrocytes can be used to study the process of myelin membrane assembly and organization. Furthermore, the "flat myelin" model allows studying domain formation in the plasma membrane of myelinating cells in a two-dimensional system, easily accessible for light microscopy applications.

\section{Lipids in Myelin Domains of pOLs}

Characterization of myelin-like domains in primary oligodendrocytes presented above shows polarized distribution of proteins in the plasma membrane of pOLs, and raises the question how this plasma membrane asymmetry is achieved. The main driving forces for compartmentalization of the plasma membrane are cytoskeleton-based (mesh work model), and lipid-based (microdomain or phase model) [226]. J. Zimmermann (AG Simons) demonstrated that depolymerisation of microfilaments or microtubules did not lead to redistribution of MAG and CNPase into MBP-positive domains in mature oligodendrocytes. Therefore, it is unlikely that the actin or tubu- 
a

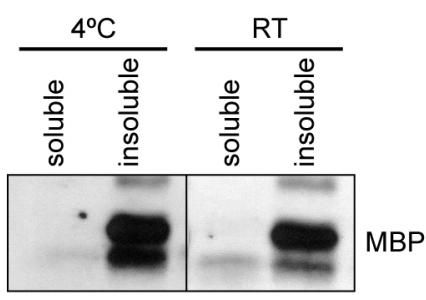

b

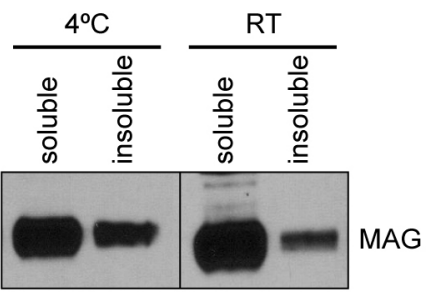

AG
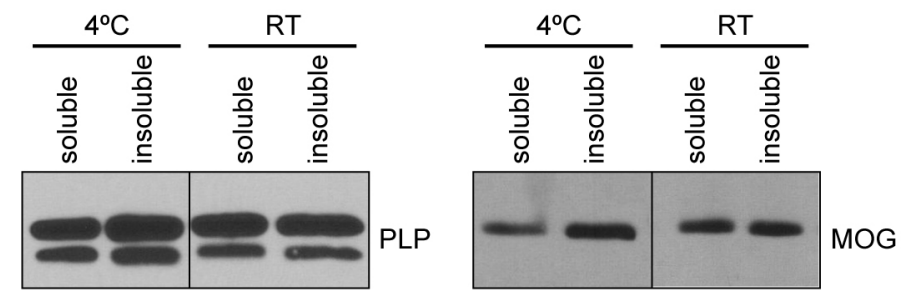

Figure 3.24: CHAPS solubility of myelin proteins MBP, PLP, MOG and MAG.

Detergent solubility assay was performed with myelin preparations at room temperature (RT) and at $4^{\circ} \mathrm{C}$. Soluble and insoluble fractions were separated by centrifugation and analyzed by Western blotting with antibodies against MBP, PLP, MOG and MAG. Out of proteins examined, MAG showed the highest solubility in CHAPS.

lin cytoskeleton are involved in selective retention of MAG and CNPase within the processes of oligodendrocytes.

The other possibility for plasma membrane organization comes from differential interactions between its components, especially sphingolipids and cholesterol, which can lead to local membrane condensation and domain formation. To analyze whether the compact and non-compact myelin proteins interact with different lipid components in the membranes, we performed detergent solubility tests on myelin isolated from the CNS of wild-type mice. As revealed by immunoblotting, the compact myelin proteins MBP, PLP and MOG were less soluble in a zwitterionic non-denaturing detergent CHAPS, than the non-compact myelin protein MAG (see Figure 3.24). Distinct detergent solubility suggests that these proteins have different lipid microenvironments.

To characterize the lipid composition of the pOLs membrane domains, we performed lipid analysis of heavy and light membrane fractions from primary oligoden- 


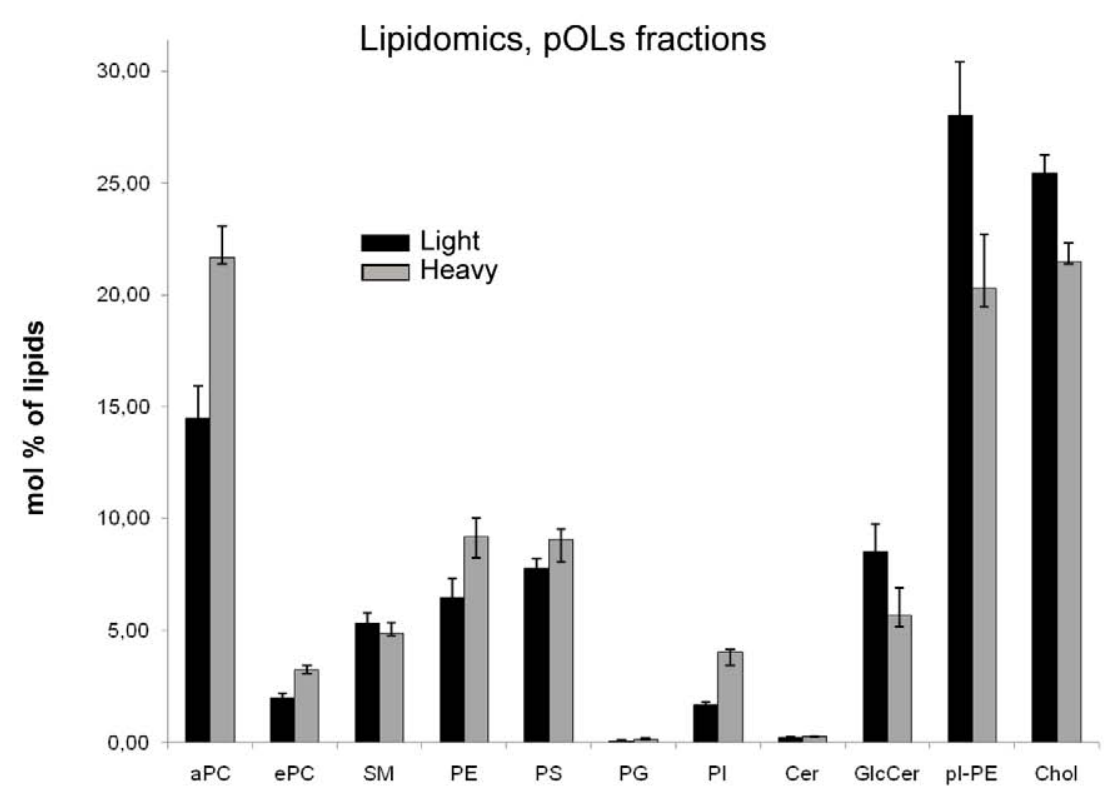

Figure 3.25: Analysis of lipid classes in membrane subfractions from pOLs cultures.

Black and gray bars correspond to light and heavy membrane fractions respectively. Bars represent means \pm SD from three independent experiments. PC, phosphatidylcholine; SM, sphingomyelin; PE, phosphatidylethanolamine; PS, phosphatidylserine; PG, phosphatidylglycerol; PI, phosphatidylinositol; Cer, ceramide; GlcCer, glycosylceramide; pl$\mathrm{PE}$, plasmenylethanolamine (plasmalogen PE); Chol, cholesterol.

drocytes. Biochemical separation of pOLs membranes in a sucrose density gradient was performed as documented in Section 2.2.2. Light fractions (low-density) were collected from the $0.32 / 0.62 \mathrm{M}$ sucrose interface, heavy fractions (high-density) were collected from the $0.7 / 1 \mathrm{M}$ sucrose interface. This separation procedure results in acquiring membrane fractions with distinct protein compositions (with non-compact myelin protein MAG being restricted to the heavier fraction, and compact myelin proteins being recovered from all fractions, as described in Section 3.1.2). Lipidomics analysis of heavy and light fractions from pOLs plasma membrane showed quantitative differences in lipid composition of the fractions. Mass spectrometry revealed that the lighter fractions were not only enriched in cholesterol (Chol), but also con- 


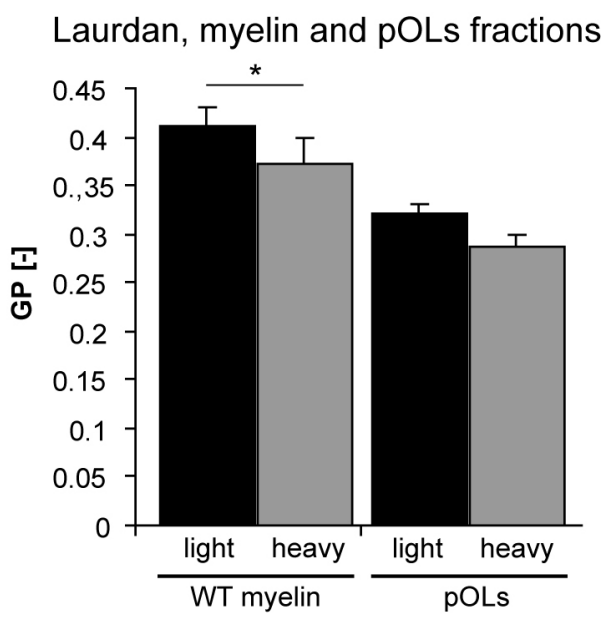

Figure 3.26: Lipid order analysis of membrane subfractions from isolated myelin and from cultured pOLs.

C-Laurdan spectroscopy reveals higher membrane order for lighter membrane fractions from wild-type myelin and from primary oligodendrocytes. For myelin samples, bars represent means $\pm S D$; * denotes significant difference (t-test), $\mathrm{P}<0.05 ; \mathrm{n}=5$. For primary oligodendrocytes, bars show means $\pm 1 / 2$ range of values $(n=2$; cells collected from at least 8 independent preparations for each experiment).

tained higher amounts of glycosphingolipids (GlcCer) and lower amounts of phosphatidylcholine (PC) compared to the heavier fraction (see Figure 3.25). In addition, quantitative analysis of lipid molecular species revealed an increase in saturated species, at the expense of polyunsaturated species, as well as higher abundance of long-chain fatty acids in the lighter fractions (see Figure C.6 in Appendix C).

Further, we compared the lipid order in heavy and light subfractions prepared from isolated wild-type CNS myelin preparations and from cultured pOLs with a fluorescent probe C-Laurdan. For lipid order analysis, C-Laurdan fluorescent spectra were recorded. C-Laurdan undergoes a shift in its peak emission wavelength from approx. $500 \mathrm{~nm}$ in fluid membrane to $400 \mathrm{~nm}$ in condensed membranes [296]. The fluorescent intensities of Laurdan at both peak emission wavelengths were compared to obtain the normalized ratio representing the generalized polarization (GP). In CLaurdan measurements, higher GP values stand for more condensed and ordered 


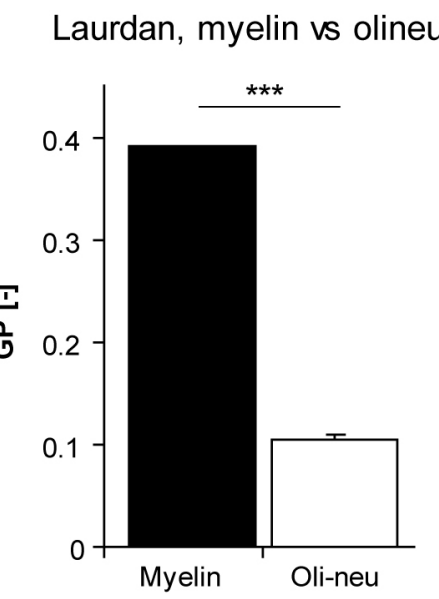

Figure 3.27: Lipid order analysis in purified myelin and in Oli-neu membranes.

C-Laurdan spectroscopy showed that purified myelin is significantly more ordered than the membranes prepared from oligodendroglial precursor cells Oli-neu. Bars represent means $\pm S D ;{ }^{* * *}$ denotes significant difference (t-test), $\mathrm{P}<0.001 ; \mathrm{n}=3$.

membranes. C-Laurdan assay revealed that both in case of myelin preparations and when pOLs membranes were used, the lighter membrane fractions have substantially higher GP values than the heavier ones. This indicates that the lighter membrane fractions have higher lipid order (see Figure 3.26).

Total membranes isolated from oligodendroglial membrane precursor cells (Olineu) had almost 4-fold lower GP values than the purified myelin (Figure 3.27). This demonstrates that myelin is a highly condensed membrane and suggests a possibility that self-organization of lipid phases could underlie lipid sorting in myelin membrane.

To assess whether myelin lipids are indeed able to separate without a requirement for proteins, experiments in giant unilamellar vesicles (GUVs) were performed. Lipids were isolated from purified or crude myelin and oligodendroglial cell membranes (total membrane preparation from Oli-neu cells, as described in Section 2.2.2). Strikingly, when lipids were mixed in a 1:1 ratio, domain formation was observed in most of the GUVs (see Figure 3.28). GUVs containing only lipids of purified myelin or only lipids of oligodendroglial cell membranes did not show domain formation. 
a

WT myelin + oli-neu

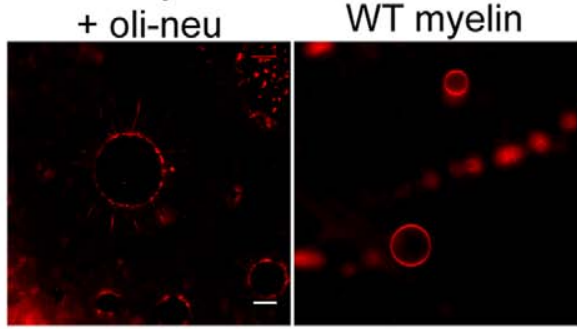

oli-neu

zoom in:

CerS2 myelin

+ oli-neu

Cers2 myelin

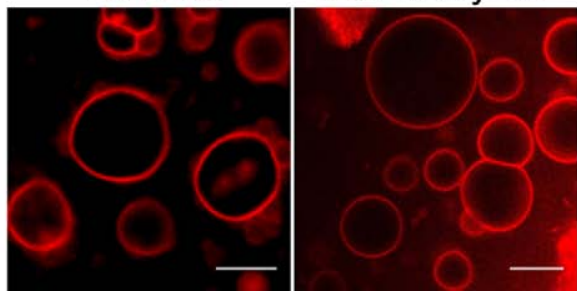

C Shiv myelin

+ oli-neu
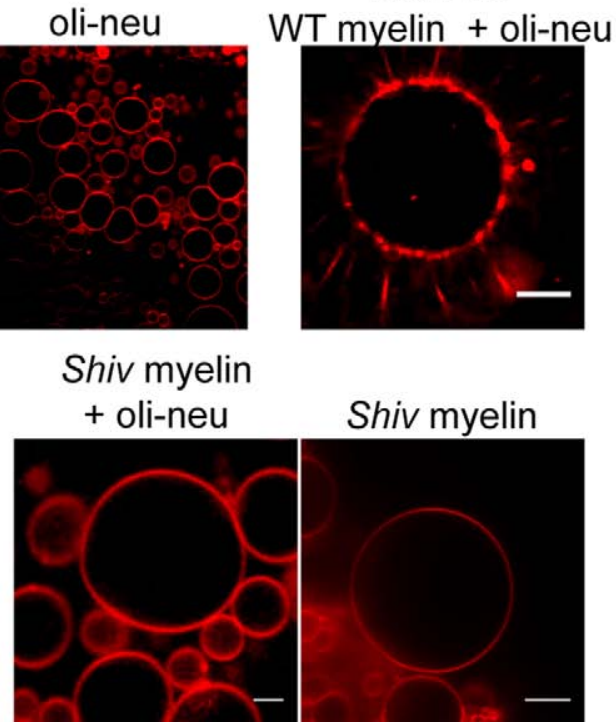

Shiv myelin

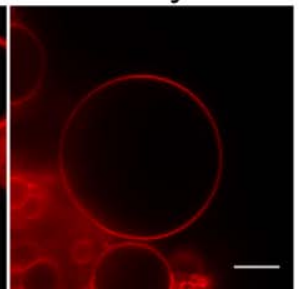

Figure 3.28: Self-organization of myelin lipids results in lateral segregation in model membranes.

In vitro assay of domain formation in giant unilamellar vesicles (GUVs) is shown.

(a) GUVs were prepared using 1:1 lipid mixtures from total Oli-neu membranes and wildtype (WT) or mutant myelin. DiD $(0.1 \%)$ was used to mark the liquid disordered phase. Clear domains were formed when WT myelin lipids were combined with Oli-neu lipids. In uncombined GUVs, neither lipids from myelin preparations, nor lipids from Oli-neu membranes formed domains.

(b and c) No phase separation was observed when crude myelin from Shiverer and CerS2 knock-out mice were used. Scale bar, $10 \mu \mathrm{m}$.

As lipid order and potential for self-assembly (as shown above) can be involved in myelin membrane domain formation, we further sought to analyze domain organization in plasma membrane of oligodendrocytes with manipulated lipid composition. 


\subsubsection{Depletion Studies on Myelin Membrane Organization in pOLs and in CNS Myelin}

\section{Sphingolipid Depletion Results in Intermixing of Membrane Domains in pOLs}

In previous sections we have shown that primary oligodendrocytes develop distinct plasma membrane domains, which can be observed with microscopy and separated biochemically. These domains differ in protein and lipid composition, as well as in lipid order. It has been reported that enrichment in sphingolipids can lead to an increase in lipid order in biological membranes [221]. We showed that in addition to being abundant in myelin, sphingolipids are enriched in lighter membrane subfractions of pOLs (see Figure 3.25), which have higher lipid order than the heavier subfractions (see Figure 3.26). These findings suggest that myelin sphingolipids may contribute to the lateral organization of myelin membrane. To assess whether sphingolipids are involved in myelin membrane domain assembly, we treated primary oligodendrocytes with fumonisin B1 (FB1), an inhibitor of ceramide synthase and de novo sphingolipid biosynthesis [320].

Under normal culture conditions, non-compact myelin proteins MAG and CNPase are restricted to the processes of oligodendrocytes (see Figure 3.1 and Figure 3.23). With confocal microscopy, we detected that oligodendrocytes cultured with FB1 show more homogeneous plasma membrane distribution of MAG and CNPase (Figure 3.31). Furthermore, these changes did not associate with cytoskeletal rearrangements (shown by J. Zimmermann, AG Simons). The effects of FB1 treatment can be rescued by addition of brain cerebrosides (demonstrated by S. Aggarwal, AG Simons), which confirms the specificity of the FB1 effect. Thus, morphological domain assembly and/or their maintenance in pOLs requires sphingolipids.

As an alternative approach to compare domain organization under sphingolipid depletion conditions, we prepared membrane subfractions from cultured oligodendrocytes. The FB1 treatment reduced the abundance of light myelin membranes (quantified by densitometric analysis of PLP signal on Western blot, see Figure 3.29). This indicates that depletion of sphingolipids increases membrane density in pOLs, thus making their characteristic "low density" property less profound. 


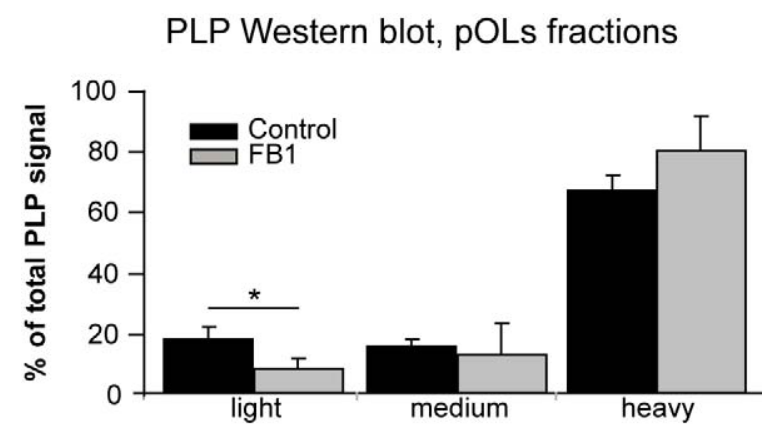

Figure 3.29: Redistribution of PLP signal in membrane subfractions from pOLs treated with FB1: densitometric analysis of Western blots.

Quantification of Western blot analysis of subfractionated membranes from mouse primary oligodendrocytes, treated with FB1 for 4 days (50 $\mu \mathrm{M}$, added every $48 \mathrm{~h}$ ), is shown. Equal volumes of light, medium and heavy fractions were collected from a 3-step density gradient (0.32/0.62/0.8 M sucrose) and subjected to SDS-PAGE and PLP immunoblotting. Densitometric analysis of fractions from control and FB1 treated oligodendrocytes showed minor decrease in PLP signal in the light fraction. t-test; ${ }^{*}, \mathrm{P}<0.05 ; \mathrm{n}=3$.

Therefore, sphingolipid depletion in cultured primary oligodendrocytes results in morphologically notable intermixing of domains on pOLs' plasma membrane and changes the physical properties of oligodendroglial membranes.

\section{CerS2 Deletion Changes Physical Properties of Myelin Membrane}

To analyze the effects of sphingolipid depletion in vivo, we performed experiments in ceramide synthase 2 deficient mice (CerS2 KO), a generous gift from A. Futerman [321, 322]. (Dihydro)ceramide synthase 2 (CerS2) is the most abundantly expressed member of the ceramide synthase gene family, expression of which transiently increases during the period of active myelination [323]. CerS2 KO mice show deficiency in synthesis of very long acyl chain (C22 - C24) ceramides, progressive loss of myelin stainability, and partial loss of compacted myelin [61].

We isolated crude myelin fractions from the brains of CerS2 KO mice and evaluated their lipid order and diffusion properties. With Laurdan spectroscopy we found that CerS2 KO crude myelin has significantly lower GP values than the crude myelin 
a Laurdan, crude myelin

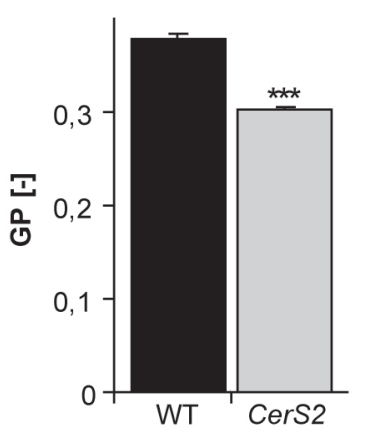

b FCS, crude myelin

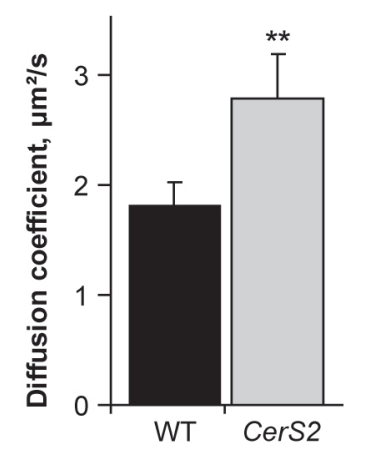

Figure 3.30: Membrane order and diffusion properties of CerS2 KO myelin.

(a) C-Laurdan fluorescence spectroscopy detected significantly lower GP values in crude CNS myelin fractions, prepared from CerS2-deficient (CerS2) mice, than in crude myelin isolated from the wild-type (WT) littermates (t-test; ${ }^{* * *}, \mathrm{P}<0.001 ; \mathrm{n}=3$ ).

(b) Fluorescence correlation spectroscopy (FCS) revealed significant increase in translational diffusion in crude myelin, isolated from the brains of CerS2 KO mice, in comparison to crude myelin fractions purified from the brains of WT littermates (t-test; ${ }^{* *}, \mathrm{P}<0.01 ; \mathrm{n}$ $=8)$.

Graph bars represent mean values \pm SD.

fractions isolated from the brains of the WT mice. Lower GP values indicate lower lipid order in myelin from animals deficient for CerS2 enzyme (see graph (a), Figure 3.30).

Diffusion properties of CerS2 KO myelin were analyzed with fluorescence correlation spectroscopy (FCS). FCS measurements showed a significant increase in diffusion coefficient values in CerS2 myelin preparations in comparison to myelin preparations from the WT mice, as plotted in graph (b), Figure 3.30.

Further we performed in vitro assays for domain formation.

The GUVs constituted with combined lipid extracts from crude CerS2 KO myelin and the Oli-neu membrane preparation did not comprise separated lipid phases (see panel (b), Figure 3.28). In contrast, lipids extracted from crude WT myelin preparation (WT littermates of CerS2 KO mice were used) demonstrated 


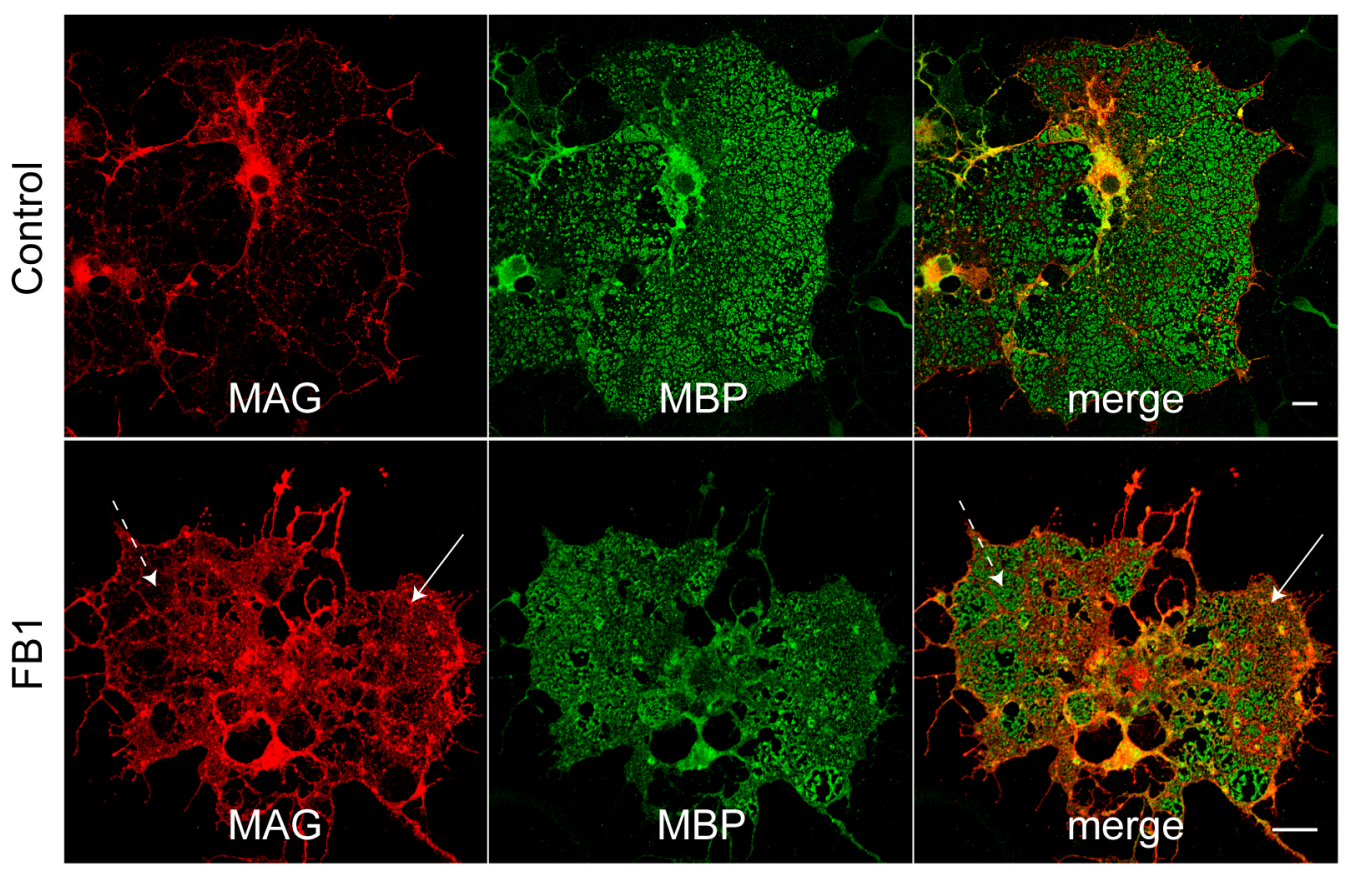

Figure 3.31: Depletion of sphingolipids results in MAG redistribution in primary oligodendrocytes.

Co-immunostaining for MAG (red) and MBP (green) in control and FB1 treated (50 $\mu \mathrm{M}$, added every 48 h, 4 days) pOLs is shown. In control cells, MAG was restricted to cell bodies and processes, whereas in FB1 treated cells MAG is reallocated into membrane sheets (white arrow). Nevertheless, MAG does not partition into plasma membrane areas with higher intensities of MBP signal (green) (dashed arrow). Scale bar, $10 \mu \mathrm{m}$.

clear domain formation when mixed with Oli-neu membrane lipids in GUVs (shown in panel (a), Figure 3.28).

The characteristics of CerS2 KO myelin are consistent with the changes observed in the plasma membrane of the FB1 treated pOLs. This data confirms the role of sphingolipids in defining myelin membrane order and diffusion properties.

\section{Sphingolipid Depletion Decreases MBP Production by pOLs}

We have shown that sphingolipids are required for high lipid order and slow diffusion characteristics of the myelin membrane, as well as for its lateral organization. 
However, in addition to this, we observed changes in MBP immunoreactivity in pOLs plasma membrane in sphingolipid-depleted cultures. FB1 treatment of primary oligodendrocytes not only led to redistribution of MAG and CNPase into membrane sheets, but also lowered the intensity of MBP signal in the sheets. Furthermore, despite that MAG and CNPase were more spread on the pOLs membrane sheets (white arrow, Figure 3.31), intermixing of MAG/CNPase and MBP-positive domains was nevertheless often avoided (dashed arrow, Figure 3.31). This suggests that the observed changes in protein distribution under sphingolipid depletion conditions might be to some extent mediated through MBP.

To assess whether depletion of sphingolipids affects the expression of MBP, we determined protein amounts in FB1 treated cultures by Western blotting. We detected a striking reduction in levels of myelin basic protein, but not of several other myelin proteins (MAG, CNP and PLP) after FB1 treatment (see Figure 3.32). However, with real-time PCR analysis we did not observe significant changes in MBP mRNA levels (see Figure 3.33). In experiments using cycloheximide to prevent new protein synthesis, faster degradation of MBP was observed in FB1 treated cells (as shown in Figure 3.34), indicating that sphingolipids may regulate MBP stability. These data suggest a possible role of sphingolipids in regulation of MBP protein levels, thus facilitating assembly of the plasma membrane domains in oligodendrocytes.

\section{MBP Deletion Interferes with pOLs Membrane Domain Organization}

To assess whether MBP is involved in domain organization of myelin membrane, we used Shiverer mice, a naturally occurring mutant lacking functional myelin basic protein due to a large deletion in the MBP gene. These mice display severe hypomyelination and seizure behavior, which increases in frequency during the animal's shortened lifespan [75]. Despite the lack of MBP, shiverer oligodendrocytes develop normally in culture, and generate normal flat membrane sheets after 3 - 4 days [297]. Form Shiverer mice, we prepared cultures of primary oligodendrocytes and isolated crude CNS myelin fractions.

Localization of the non-compact myelin proteins CNPase and MAG, normally confined to the processes and cell bodies of cultured oligodendrocytes, was disturbed in Shiverer pOLs. The observed phenotype was more severe than in case of sph- 


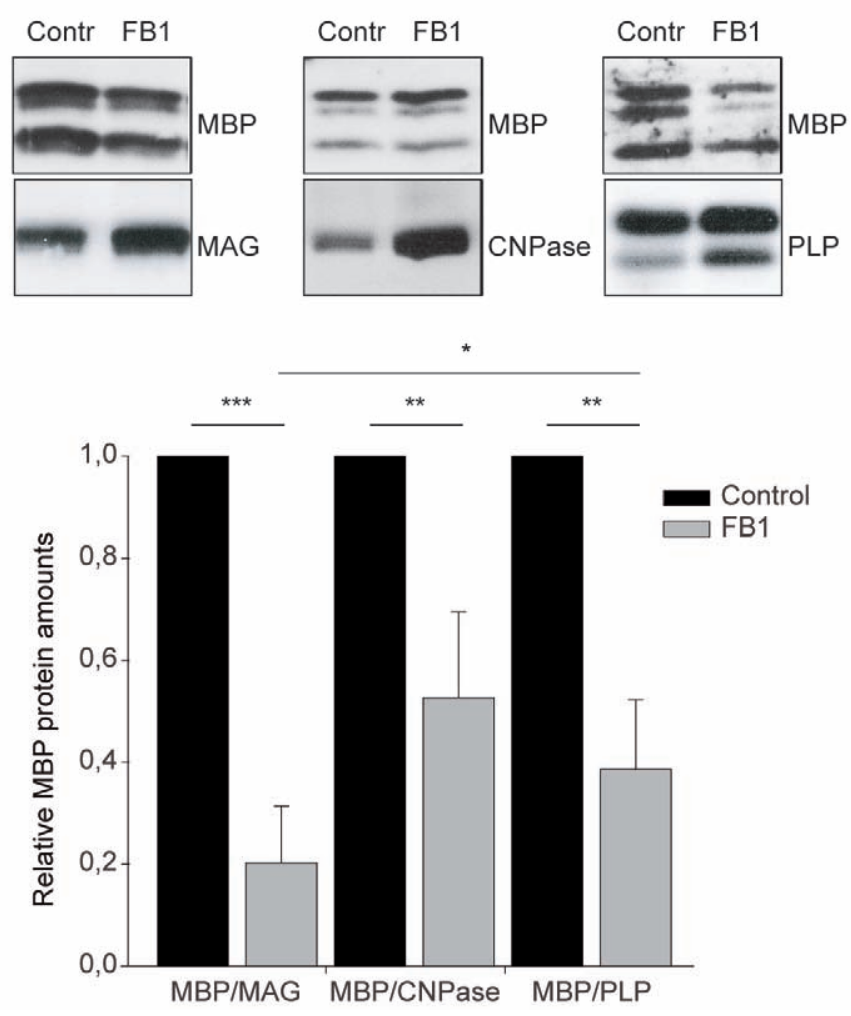

Figure 3.32: Sphingolipid depletion causes reduction of MBP amounts in pOLs.

Primary oligodendrocytes were treated with FB1 (50 $\mu \mathrm{M}$, added every $48 \mathrm{~h}, 7$ days), lysed and the amounts of myelin proteins MBP, PLP, CNPase, and MAG were determined by Western blotting. For normalization, MBP amounts were plotted as relative ratios as compared to MAG, CNPase and PLP. Sphingolipid depletion results in significant reduction of relative amounts of myelin basic protein in oligodendroglial cultures (Mann-Whitney rank sum test); * $\mathrm{P}<0.05 ;{ }^{*}, \mathrm{P}<0.01 ; \mathrm{n}=6$ - 8. Graph bars represent mean values $\pm \mathrm{SD}$, black bars refer to control values, gray bars represent values for FB1 treated cells.

ingolipid depletion (FB1 treatment). Immunofluorescence stainings for MAG and CNPase demonstrated dispersed distribution of these non-compact myelin proteins over the plasma membrane of Shiverer pOLs. In contrast, in WT cells, these proteins are restricted to cell bodies and processes of oligodendrocytes (confocal images are presented in Figure 3.35, quantifications are shown in Figure 3.36). 


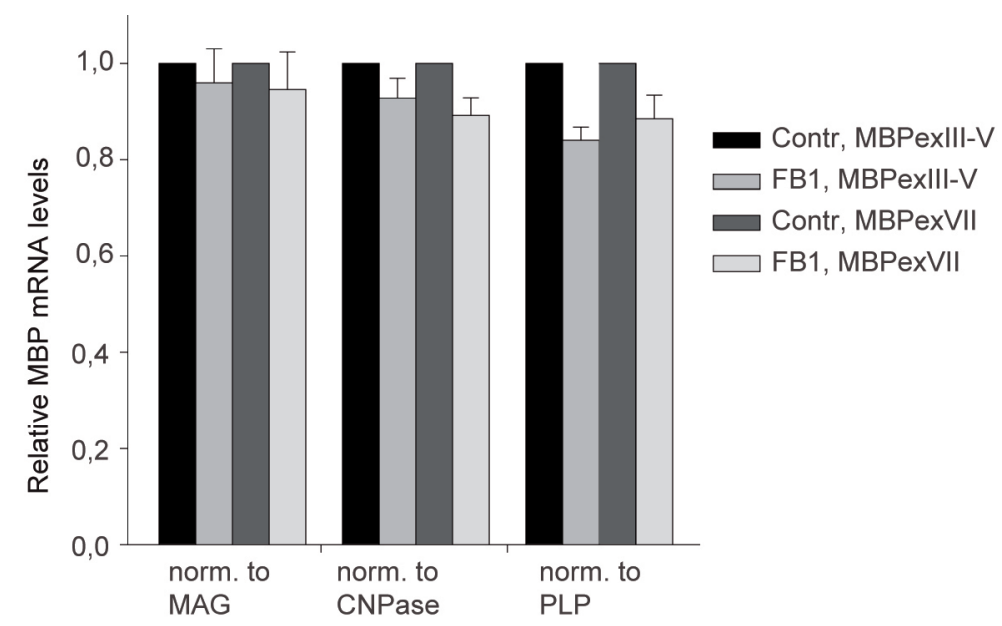

Figure 3.33: Relative MBP expression levels in pOLs after FB1 treatment.

Real-time PCR was performed on cDNA samples produced from total mRNA extracts of control and FB1 treated (50 $\mu \mathrm{M}$, added every $48 \mathrm{~h}, 7$ days) primary oligodendrocytes. Relative abundance of MBP mRNA in the samples was quantified by $\Delta \Delta \mathrm{C}_{T}$ method [275], which involves double normalization of Ct values: the expression of a gene of interest is normalized to the expression of a housekeeping gene and the treated samples are normalized to the control samples. In this case, expression levels of the several myelin genes were used (MAG, CNPase and PLP) for normalization, in order to compensate for cellular heterogeneity of the mixed oligodendroglial cultures. Two different primer pairs were used for MBP to cover the majority of isoforms. No significant difference between the samples could be detected. Bars represent means $\pm S E M ; n=6$; for each experiment measurements were done in triplicates.

To test if the different plasma membrane organization observed in Shiverer oligodendrocytes is caused by the cytoskeleton rearrangement, we compared microfilament and microtubules distribution in WT and Shiverer pOLs. In oligodendrocytes lacking MBP, microfilaments and micrutubules did not show noticeable reallocation into membrane sheets (see Figure 3.39). Filamentous actin (stained with phalloidin-Rhodamine) looked indistinguishable in the WT and Shiverer oligodendrocytes (panel (a), Figure 3.39), although phalloidin signal was difficult to detect in mature pOls (as described in Section 3.2.1). 


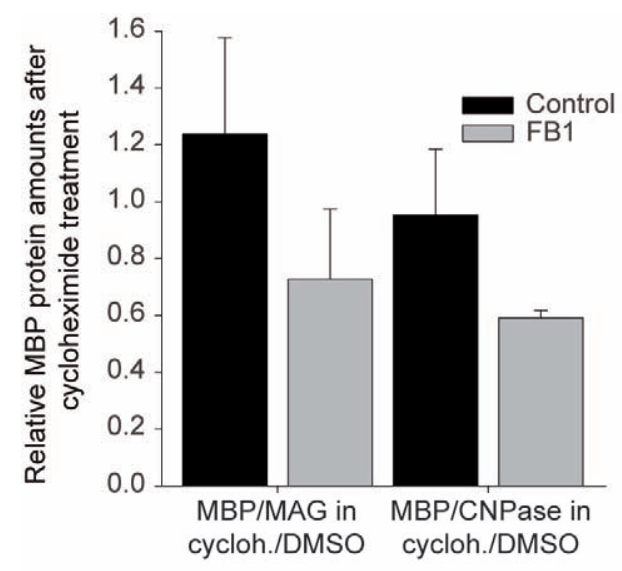

Figure 3.34: MBP protein stability is reduced in sphingolipid depletion conditions.

Primary oligodendrocytes were treated with FB1 (50 $\mu \mathrm{M}$, added every $48 \mathrm{~h}, 7$ days), and subsequently treated with cycloheximide $(50 \mu \mathrm{g} / \mathrm{mL}, 6 \mathrm{~h})$ to block new protein synthesis. The amounts of MBP, CNPase, and MAG was determined by Western blotting. For normalization, MBP amounts are presented as relative ratio to MAG and CNPase amounts. Treatment with cycloheximide reduces relative levels of MBP in FB1 treated cells (gray bars) to a larger extent than in FB1-untreated cells (black bars) indicating faster degradation of MBP under sphingolipid depletion conditions. Mann-Whitney rank sum test on normalized to control data shows significant difference between control and FB1 treated $\mathrm{pOLs} ; \mathrm{P}<0.05 ; \mathrm{n}=3$. Bars represent means $\pm \mathrm{SD}$.

Tubulin staining showed a broader variation within the population of Shiverer oligodendrocytes. In the majority of mature Shiverer pOLs, microtubules exhibited a pattern of distribution similar to that observed in WT pOLs (panel (b), Figure 3.39). However, in a minor portion of Shiverer pOLs with enlarged cell bodies and small sheets, microtubules were more dispersed.

Further on we performed a biochemical assay to compare Shiverer and WT crude myelin preparations. Subfractionation was carried out in sucrose density gradients according to the protocol described in Section 2.2.2. Western blot analysis revealed a substantially more intense PLP signal in the heavier subfractions of Shiverer myelin, as compared to the WT myelin (see Figure 3.37). These changes in physical properties of myelin under MBP deletion conditions are similar to the ones observed in 

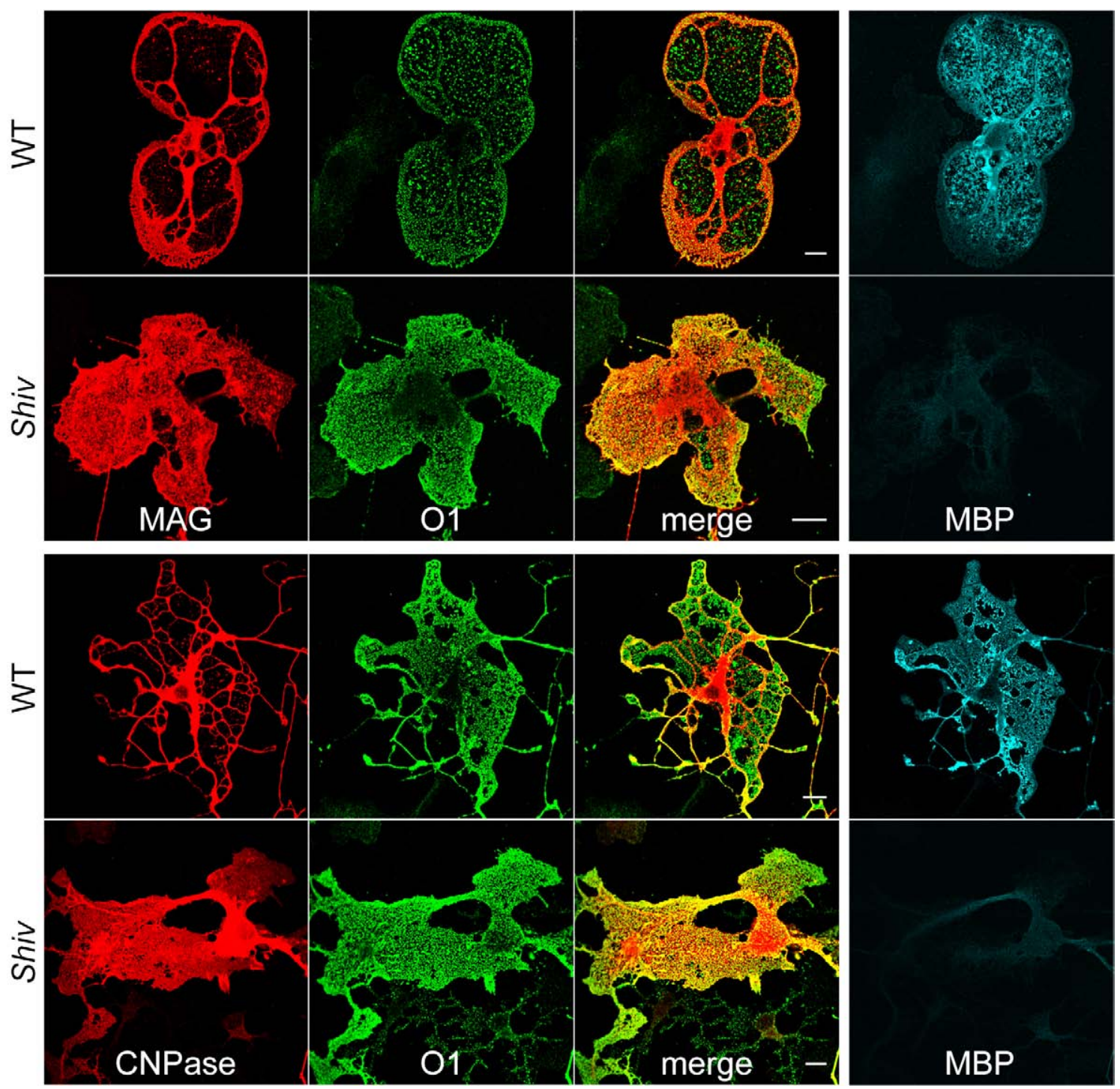

Figure 3.35: MBP deficiency results in loss of membrane asymmetry in primary oligodendrocytes: MAG and CNPase redistribution in Shiverer pOLs.

Immunostaining for MAG (red), CNPase (red), O1 (green) to mark the membranes of primary oligodendrocytes, and MBP (cyan) to distinguish MBP-deficient (Shiv) oligodendrocytes from the wild-type (WT) oligodendrocytes is shown. Merge images represent overlay of MAG or CNPase stainings with $\mathrm{O} 1$ signal to visualise distribution of MAG and CNPase in the membrane sheets of pOLs. In Shiverer oligodendrocytes, dramatic reallocation of MAG and CNPase into membrane sheets was observed. Scale bar, $10 \mu \mathrm{m}$. 

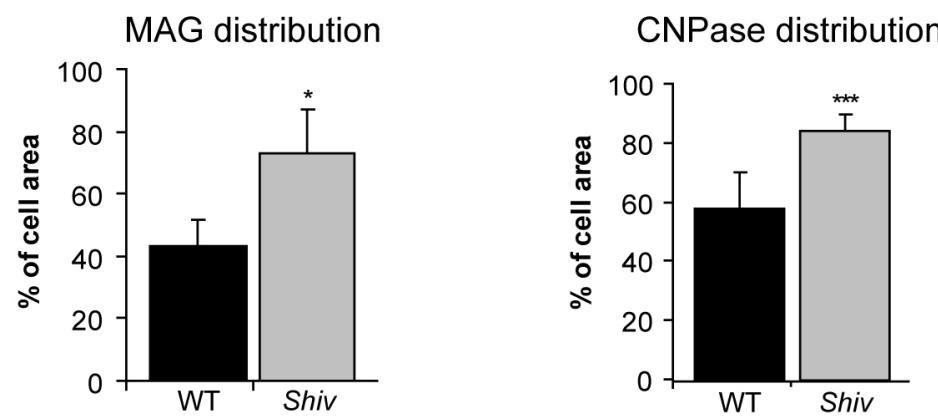

Figure 3.36: Quantitative analysis of MAG and CNPase distribution in Shiverer pOLs. Quantification of the relative surface area occupied by the non-compact myelin proteins MAG and CNPase in MBP-deficient (Shiv) and wild type (WT) oligodendrocytes is displayed. MAG and CNPase stainings show significantly broader distribution in Shiverer pOLs, than in the WT pOLs (t-test); ${ }^{*}, \mathrm{P}<0.05$; ${ }^{* *}, \mathrm{P}<0.001 ; \mathrm{n}=20-21$. Graph bars represent mean values $\pm S D$; black and gray bars refer to values obtained from control and Shiverer oligodendrocytes respectively.

the membranes of pOLs under sphingolipid depletion conditions (as shown above in Figure 3.29).

To analyze membrane organization in MBP-deficient myelin, C-Laudran and fluorescence correlation spectroscopy (FCS) measurements were performed.

C-Laurdan assay for evaluation of lipid order in myelin fractions revealed that GP values are significantly lower in crude myelin preparations from Shiverer mice than in the preparations from the WT animals (displayed in graph (a), Figure 3.38), indicating lower membrane order in Shiverer myelin. This data shows that lipid order is affected by MBP deletion, similar to the observations made for CerS2 deletion (shown in graph (a), Figure 3.30). Reduction in lipid order seemed even more profound in Shiverer samples.

To characterize the diffusion properties of MBP-deficient myelin, fluorescence correlation spectroscopy (FCS) analysis was carried out on crude myelin preparations from Shiverer brains. Translational diffusion in Shiverer myelin was faster, as compared to the WT myelin (shown in graph (b), Figure 3.38), resembling the 
a PLP Western blot, myelin fractions

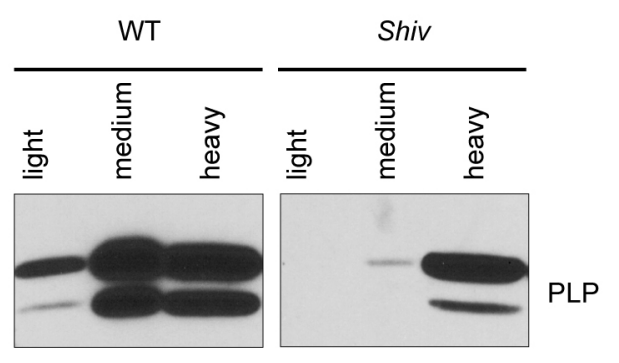

b PLP Western blot, myelin fractions (quantification)

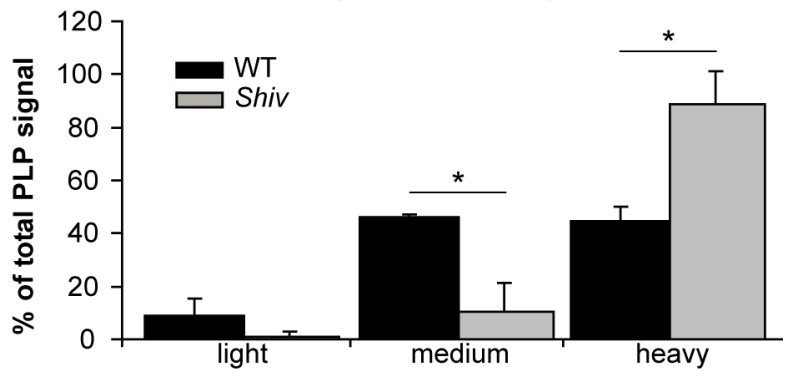

Figure 3.37: Redistribution of PLP signal in Shiverer crude myelin subfractions.

(a) Crude myelin isolated from the brains of the wild-type (WT) and MBP-deficient (Shiv) mice was further separated into light, medium and heavy subfractions, collected from the $0.32 / 0.62,0.62 / 0.7$ and $0.7 / 1 \mathrm{M}$ sucrose interfaces, respectively. Equal volumes of the fractions were resolved by SDS-PAGE and analyzed by immunoblotting with 3F4 antibodies against PLP.

(b) Densitometric quantification of immunoblots showed considerable redistribution of the PLP signal into heavier fractions in Shiverer preparations. t-test; * $\mathrm{P}<0.05 ; \mathrm{n}=3$. Graph bars represent mean values $\pm S D$; black and gray bars refer to values obtained from the WT and Shiverer preparations, respectively.

changes observed in the sphingolipid-depleted myelin (shown in graph (b), Figure 3.30).

To test whether the lipids extracted from Shiverer myelin are able to separate in model membranes, as we could observe for the preparations from the WT animals (see panel (a), Figure 3.28), experiments in giant unilamellar vesicles (GUVs) were performed. When lipids from crude preparations of MBP-deficient myelin were mixed with lipid extracts from oligodendroglial cell membranes, domain formation was not observed, resembling the homogeneous GUVs obtained with CerS2 KO samples.

Taken together, MBP is required for lateral organization, high order and low diffusion properties of myelin membrane. With respect to MAG and CNPase localization in pOLs plasma membrane, Shiverer oligodendrocytes display a dramatic dispersion phenotype, which is even more striking than the one observed 
a Laurdan, crude myelin

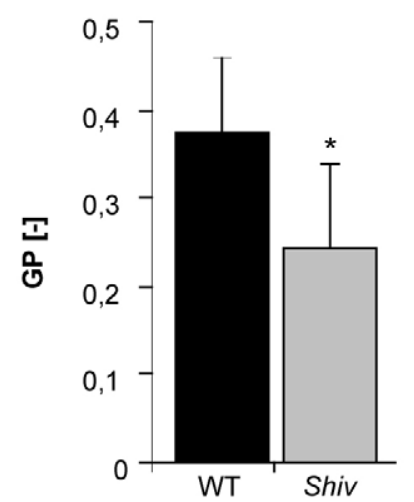

b FCS, crude myelin

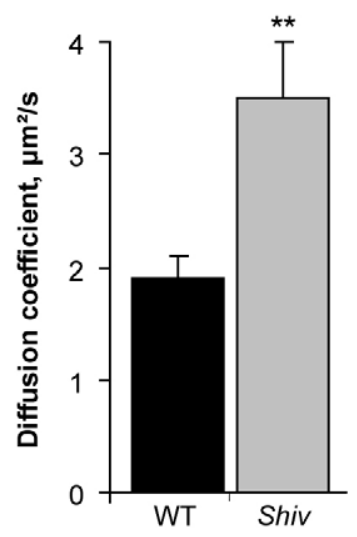

Figure 3.38: Membrane order and diffusion properties of MBP-deficient (Shiverer) crude myelin.

(a) C-Laurdan fluorescence spectroscopy revealed significant decrease in GP values in crude CNS myelin fractions, prepared from MBP-deficient (Shiv) mice, as compared to crude myelin, isolated from the wild-type (WT) littermates (t-test); ${ }^{*}, \mathrm{P}<0.05 ; \mathrm{n}=6$.

(b) Fluorescence correlation spectroscopy (FCS) showed significantly faster translational diffusion in Shiverer crude myelin, than in the WT crude myelin (t-test); ${ }^{* *}, \mathrm{P}<0.01 ; \mathrm{n}=8$ $-10$.

Graph bars represent mean values $\pm S D$; black and gray bars refer to values obtained from the WT and Shiverer preparations respectively.

in sphingolipid-depleted cells. In line with the changes detected for sphingolipid depletion, MBP deletion leads to increase in diffusion coefficient in the membranes and decrease in lipid order.

The data described above indicate that MBP and sphingolipid deficiencies result in similar changes in myelin membrane characteristics due to regulation of MBP protein amounts by sphingolipids (possibly though influencing MBP stability). MBP, the amounts of which are regulated by sphingolipids, is necessary for myelin membrane polarization at the protein level. Furthermore, myelin lipids have potential for self-organization and segregate from lipids of oligodendroglial membranes by a 
a
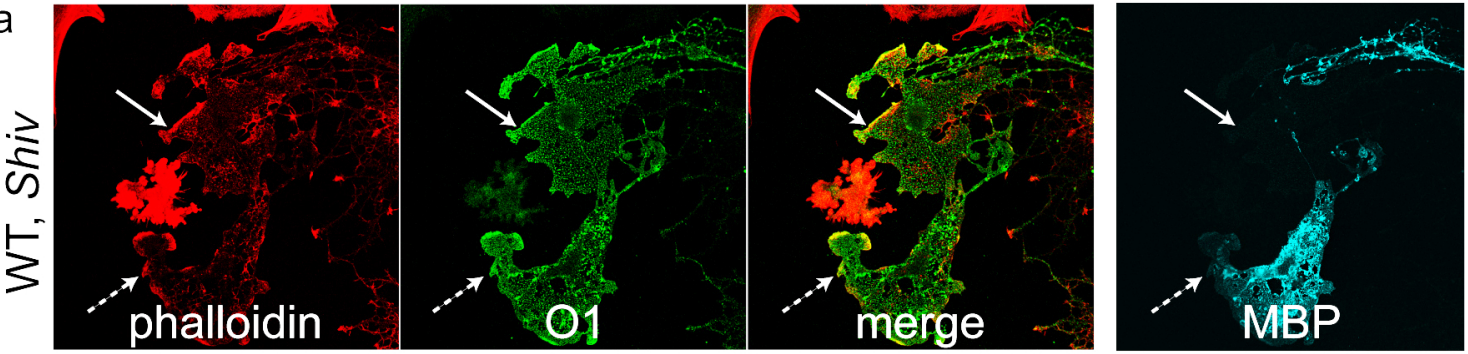

b
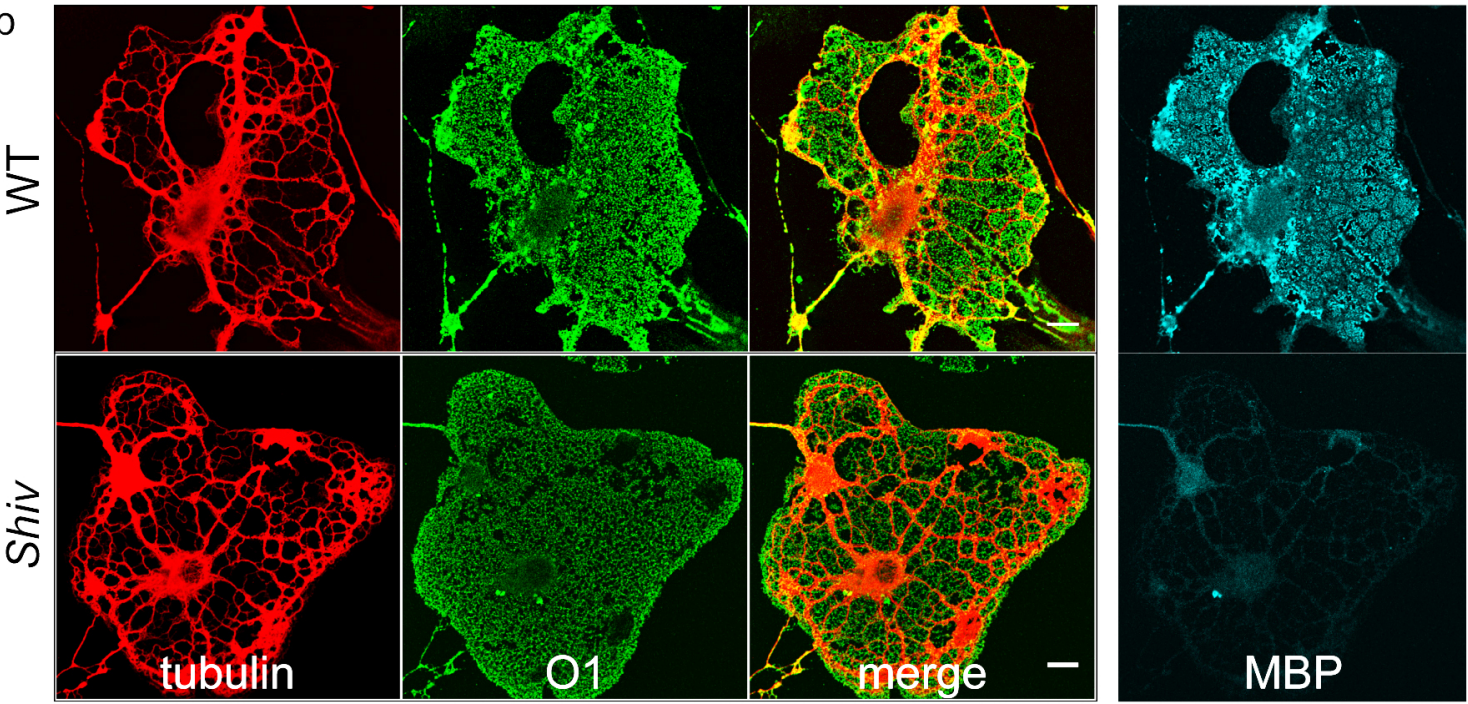

Figure 3.39: Cytoskeleton in Shiverer pOLs.

Mixed cultures of primary oligodendrocytes containing MBP-deficient (Shiv, white arrow) and wild-type (WT, dashed arrow) cells were stained for filamentous actin (panel (a), phalloidin, red), microtubules (panel (b), tubulin, red), galactosylceramide (O1, green) to visualize membrane sheets, and MBP (cyan) to distinguish MBP-positive and MBPnegative pOLs. In oligodendrocytes lacking MBP, microfilaments and micrutubules were not redistributed into membrane sheets. Scale bar, $10 \mu \mathrm{m}$.

phase separation process. Under conditions of reduced sphingolipid content, phase separation of myelin lipids did not occur. 


\section{Chapter 4}

\section{Discussion}

\subsection{Cell Culture Model to Study Myelin Sheath: Model Validation}

In this Section we discuss advantages and limitations of our novel model for studying myelin growth. The first Subsection focuses on the analysis of the morphological traits of the cellular model system, whereas the second Subsection evaluates the biochemical characteristics of the model.

\subsubsection{Morphological Approach}

Myelin has been extensively studied throughout the last decades, but the most fundamental questions about its assembly and growth have remained unsolved [24]. Partially, this is due to the tight packing of myelin membrane layers in vivo, which makes it hardly assessable by conventional light microscopy. To overcome this restriction, we have established a two-dimensional model of an "unrolled" myelin sheath, making use of cultured primary oligodendrocytes (pOLs). Oligodendroglial cultures can be prepared in different variations: pure and mixed, from embryonic and neonatal brains, spinal cord and neurospheres [324]; some investigations have been performed on cultured oligodendrocytes which develop multiple processes and sheets [205, 325], in other projects myelinating cocultures or organotypic cultures were employed [326, 327]. 
Under the culture conditions used in this study (see Section 2.2.3), mouse oligodendrocytes produce flat membrane extensions, which mimic the biochemical and morphological microcomposition that myelin acquires in vivo. In this work we describe that plasma membrane of cultured primary oligodendrocytes is polarised, i.e. pOLs develop distinct membrane domains, resembling compact myelin and noncompact myelin formed in vivo. Surprisingly, these domains develop their identity even in cell culture without the requirement for wrapping around axons, which argues in favor of yet uncovered intrinsic cellular mechanisms underlying this process, rather than neuronal signalling or other cellular context.

In vivo, myelin basic protein (MBP) and proteolipid protein (PLP) localize to the compact internodal areas of myelin and are known to be the most abundant proteins of CNS myelin. Myelin sheath is also enriched in specific sphingolipids: galactosylceramides (GalC) and sulfatides [12]. We detected that in cultured oligodendrocytes these components of compact myelin (MBP, PLP, MOG, GalC) are distributed over the myelin-like membrane sheets, whereas the non-compact myelin proteins 2', 3'cyclic-nucleotide 3'-phosphodiesterase (CNPase) and myelin-associated glycoprotein (MAG) are enriched in the cell body and processes of oligodendrocytes. In parts, this data is supported by pOLs descriptions in [325, 328].

However, being a two-dimensional model, membranes of cultured pOLs escape modulation of their polarization, which would be mediated by axons in vivo. For example, the non-compact myelin protein MAG is predominantly localized to the periaxonal lamellae of non-compact myelin in the CNS. In culture, no ab- or adaxonal specialization of oligodendrocytes takes place, thus MAG is distributed in the non-compact-myelin-like domain of pOLs with no preference to a specific segment of this area.

Another limitation of the cell culture model comes from absence of close apposition of the extracellular membrane leaflets. For this reason, restriction of protein distribution that would be in vivo imposed by the extracellular protein domains, could be circumvented. As a result, another protein MOG, which is in vivo enriched in the outer lamellae of compact myelin, in cell culture adopts more spread distribution throughout compact-myelin-like membrane domains of pOLs. 
Taken together, the cellular model reflects such important characteristics of myelin as proteolipid composition, compartmentalization of membrane into domains and compaction of MBP-enriched membrane areas, although it does not recapitulate in vivo myelin in its full complexity. The important advantages of this model are accessibility to light imaging techniques and easy manipulability, e.g. by inhibitor application, overexpression or knock-down techniques. Besides, the cell culture model simplifies interpretation of the effects observed in experiments by diminishing possible developmental compensation, which often takes place in vivo. It also attenuates the overwhelming cellular context found in brain and decreases signalling from different cell types, thus permitting the dissection of intrinsic oligodendroglial mechanisms.

\subsubsection{Biochemical Approach to Study Myelin Membrane}

In addition to the morphological approach, we employed biochemical techniques to study myelin membranes. Membrane fractions separated by density gradient centrifugation from cultured cells and from the brains of the wild-type (WT) and mutant mice (Shiverer, CerS2 KO) were further used in physical and biochemical assays performed in this work. The first step in preparation of the subfraction is isolation of crude myelin from brains and myelin-like membranes from pOLs, which relies on the large size of myelin vesicles, together with the fact that myelin has a lower density than other biological membranes [283].

In vivo, myelin is known to show regional specialization: its internodal regions consist mainly of multilamellar compact membranes, whereas paranodal, outer and inner loops of myelin, as well as inscisures are not compacted; these areas contain cytoplasm, intracellular organelles and cytoskeletal elements [12]. The population of membranes isolated in myelin preparations is heterogeneous as well. Due to this, it can be further separated into subfractions of different densities. The least dense myelin membranes (light myelin) have been previously shown to contain mainly multilamellar fragments, while the heavier myelin subfractions are enriched in single membrane vesicles and membrane fragments with two or three layers [43, 281]. These fractions have been described to have different lipid-protein ratios and dif- 
ferent protein composition, with the lighter fractions being more lipid-rich [329]. With respect to the protein composition of myelin subfractions, the compact myelin protein MBP is known to be enriched in lighter fractions, the other compact myelin protein PLP was previously shown to be distributed evenly among the fractions, whereas the non-compact myelin proteins CNPase, MAG and the components of paranodal junctions Caspr and contactin associate in higher amounts with heavier fractions [43, 202, 281, 285].

Consistent with these observations, in fractionated pOLs membranes we find MAG (protein, localized to periaxonal loops of myelin) being enriched in heavier fractions, while the compact myelin proteins PLP and MOG are distributed throughout myelin fractions of different densities. Taken together, the data from several independent studies including the one presented here indicate that the non-compact membranes from myelin and pOLs subfractionate predominantly into heaver fractions, while the compact myelin membranes (internodal multilayered myelin or myelin-like compacted areas of pOLs sheets) are enriched in the lighter fractions. This suggests that we can biochemically separate and further analyze regionally specialized myelin as well as distinct myelin-like domains of cultured primary oligodendrocytes.

\subsection{Mechanisms of Myelin Sheet Growth in pOLs}

\subsubsection{Maturation Stages in Culture}

To acquire insights in myelin sheet expansion processes, we first examined maturation stages of oligodendrocytes in culture. The stages of myelin sheet expansion were analyzed by confocal microscopy on fixed pOLs, which myelin-like sheets were resolved by immunostaining for the peripheral membrane protein MBP, a defining protein of the compact CNS myelin.

Differentiation of oligodendroglial precursor cells (OPCs) is associated with changes in cell morphology and antigen expression. In agreement with extensive literature, immature oligodendrocytes display dendritic morphology with elaborated network of processes [324, 330]. We termed this maturation stage of cultured pOLs the "spider web" stage. In studies performed in vivo, oligodendrocytes which have 


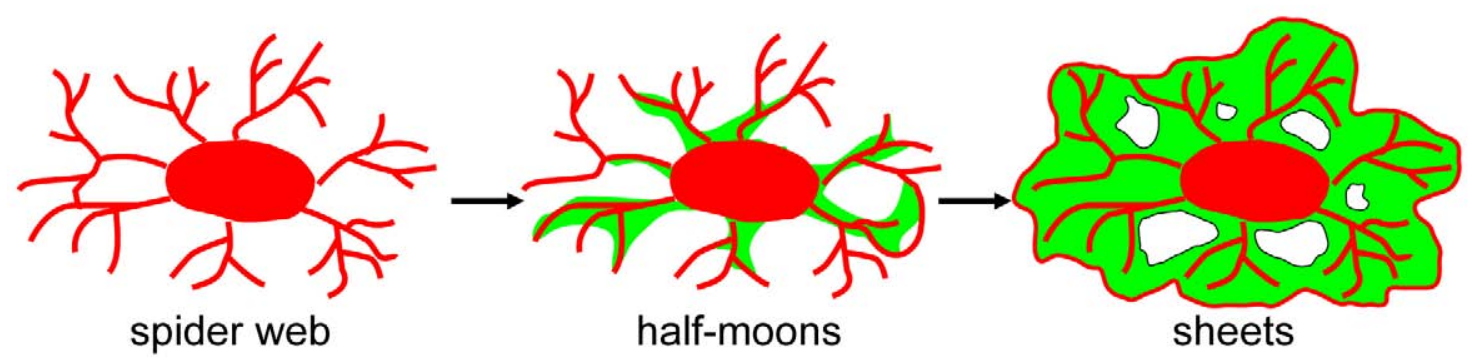

Figure 4.1: Schematic representation of myelin sheet development in culture.

"Spider web", "half-moon" and "sheet" maturation stages of primary oligodendrocytes are delineated. At the "half-moon" stage, myelin-like sheets (green) start appearing in between oligodendroglial processes (red) to fill the gaps and form spacious myelin-like sheets of the mature stage.

developed processes but not yet myelin sheets, are termed "premyelinating oligodendrocytes" [331-333]. Here we asked, how these "spider web" cells turn their processes into sheets. To resolve this, we analyzed a transitional maturation state from the "spider-web" state to the "sheets" stage.

With immunostainings on fixed and permeabilized cells we have observed a peculiar pattern of MBP staining in oligodendrocytes undergoing "spider web" to "sheets" transition. When bright MBP signals appear at the plasma membrane of oligodendrocytes, they form unusual "half-moon"-like structures (see Figure 3.9 on Page 93). To our knowledge, this transitional stage of myelin sheet development has not been described so far. According to the pattern observed, it can be anticipated, that the myelin sheets formation is carried out by filling in the gaps in between the oligodendrocytes' network of processes, as schematically shown in Figure 4.1. The "half-moon" stage is a very rapid transitional state of pOLs, which lasts only a few hours around day 3 - 4 after the OPCs were seeded on coverslips. The MBP-positive "half-moons" are compacted (almost devoid of cytoplasm, as shown with AFM, see Figure 3.20) and are lacking the cytoskeletal support (F-actin deficiency is shown in Figure 3.11 on Page 96, staining for microtubules is shown in the upper panel in Figure 3.13 on Page 99). In fact, in combination with a high enrichment in lipids 
this could promote disturbed fixation or removal of the components of this membrane during the permeabilization procedure. As MBP immunostaining requires permeabilization, this possibility should be taken into account.

To visualize the process of myelin membrane expansion and exclude the possibility of introducing artifacts due to fixation and permeabilization, we performed live imaging experiments. To mark the developing myelin-like sheets (and replace MBP immunolabeling, possible only on fixed cells) an overexpression approach was employed. The most straight-forward strategy would be to overexpress fluorescently tagged compact myelin proteins MBP or PLP. However, overexpression of compact myelin proteins has a toxic dosage effect in pOLs (overexpressed MBP-GFP aggregates in cell bodies of pOLs, as shown in Appendix C, Figure C.4), also observed in vivo [81]. To avoid the aforementioned toxicity, exogenous expression of vesicular stomatitis virus glycoprotein (VSVG) fused to a fluorescent protein was chosen for these experiments. Previously, VSVG has been demonstrated to be targeted to myelin sheets [130]. We have introduced a YFP-tagged VSVG protein into an SFV vector, followed by infection of primary oligodendrocytes and time-lapse live imaging. However, this approach was not successful due to a consistent tendency of the transmembrane proteins, fluorescently tagged on their intracellular terminus, to avoid localization in myelin-like membrane sheets (as shown in Appendix C, Figure C.4). Despite hindering interpretation of the live imaging experiments, this observation promoted further investigation of this phenomenon. We confirmed that extracellular tags did not affect protein localization in the MBP-positive domains, whereas the same proteins were excluded from the MBP-positive domains when tagged with a fluorescent protein at their cytosolic terminus (e.g. compare MOGGFP in Figure C.4 on Page 168 and myc-thr-HA-MOG in Figure 3.19 on Page 105). In addition, overexpressed soluble GFP and RFP showed very low fluorescent signal in the sheets of pOLs (see Figure C.5 on Page 169). This led us to hypothesize that the size of the intracellular domain of transmembrane proteins might regulate their partitioning into the compact-myelin-like domains of pOLs. This restriction could have developed along with compaction as a part of a specialized membrane compartmentalization mechanism in oligodendrocytes, which could have evolved to 
ensure protein exclusion from compact myelin. The size-barrier hypothesis is being further tested in our laboratory by S. Aggarwal.

With respect to the visualization of myelin sheet expansion, in future studies it would be of interest to test whether the described "half-moon"-like transition from "processes" to "sheets" can be documented with phase-contrast time-lapse live imaging approaches.

Despite the fact that the morphological relevance of the "half-moons" has not been convincingly demonstrated to date, there nevertheless are important clues to be taken from this data. The fact that the systematic "half-moon" patterns are reliably reproducible indicates differential properties within the underlying membrane and cellular structure. Boosted MBP expression, lack of the cytoskeletal support and increased lipid content of these membrane regions manifest themselves in the appearance of "half-moons".

\subsubsection{Actin Cytoskeleton in Myelin Sheet Expansion}

New insights into mechanisms of myelin sheet growth are offered by observing cytoskeletal reorganization in primary oligodendrocytes. We have revealed that the processes of immature cultured oligodendrocytes are rich in filamentous actin (Factin), whereas mature pOLs show a tremendous reduction of the F-actin signal (detected with phalloidin-Rhodamine staining, Figure 3.11 on Page 96). Furthermore, the F-actin signal is lost during the transitional "half-moon" maturation stage (described above, Section 4.2.1), when a bright MBP staining replaces the phalloidin-Rhodamine fluorescence at the branching points of the processes, where the first MBP-positive sheets appear. Although MBP seems to replace the F-actin, it is unlikely that disassembly of the microfilaments in these cells is mediated by MBP, as we observe a similar reduction of the F-actin signal in mature Shiverer pOLs, which are deficient of the myelin basic protein (compare white and dashed arrows, marking the WT and the Shiverer pOLs in Figure 3.39 on Page 131).

Previously, microfilaments have been demonstrated to form cytoarchitectural meshworks, enriched beneath the plasma membrane throughout all regions of pOLs and implemented in guiding local reorganization of microtubules [205, 334]. It has 
been shown, that processes in oligodendrocytes extend through formation of filopodia and lamellipodia, a phenomenon which employs regulation of actin nucleation and polymerisation by the proteins WAVE1 and N-WASP [209, 335]. Indeed, active regulated polymerisation of microfilaments can be expected during processes outgrowth, that takes place in immature oligodendrocytes.

In contrast, during formation of the myelin sheets, we observe tremendous reorganization and disassembly of microfilaments. In mature oligodendrocytes phalloidinRhodamine staining has a several fold lower intensity, than in non-oligodendroglial cells present in the same culture (see second image in Figure 3.11 on Page 96). Interestingly, the phalloidin-Rhodamine signal contained star-like structures, positioned in between the MBP-positive domains of the myelin-like sheets.

The cytoskeletal changes, which accompany myelin sheath expansion, wrapping and compaction have not been investigated in detail previously. Compaction of a myelin sheath implies close apposition of plasma membranes with only few nanometer distance in between, which in a way imposes a requirement for cytoskeleton retraction [13].

Said scenario is supported by reports showing transient up-regulation of gelsolin during myelinogenesis [336]. This actin-binding protein severs and caps the actin microfilaments and is possibly involved in the control of myelin wrapping [337]. Additional evidence in favor of this hypothesis is provided by in vitro experiments of Boggs et al., who showed that signalling through glycosphingolipids results in loss of the cytoskeleton [338]. In these experiments, cultured oligodendrocytes were incubated with GalC-containing liposomes to mimic the intersheath interactions in myelin, resulting in microfilament disassembly and clustering of membrane domains [213]. One more confirmation of cytoskeletal retraction comes from careful examination of figures presented in a study, which reports identification of Ermin and its colocalization with actin in mature cultured pOLs [339]. A closer look at the images reveals that the signals of both actin and Ermin are minimal in MBP- and MOG-positive sheets of cultured oligodendrocytes (see Figure 3 in [339]).

Reported induction of actin polymerization [340, 341] and microtubule bundling $[342,343]$ through interactions with MBP are in apparent contradiction with the cytoskeletal retraction hypothesis and with the requirement for myelin compaction as 
well. However, a thorough interpretation of these findings should take into account various isoforms of MBP and multiple post-translational modifications, which might modulate the reported interactions in vivo. Accompanying data on interactions of CNPase and MAG with cytoskeleton are in line with the suggested hypothesis, as both proteins localize to cytoplasm-containing non-compact areas of a developed myelin sheath, where they might contribute to organization of cytoskeleton [107, 204, 344].

Taken together, cytoskeleton retraction is most likely to take place during myelin sheath biogenesis, at latest during compaction of the myelin layers. Interestingly, our results indicate that disassembly of microfilaments might actually happen already during the primary stage of budding of MBP-positive myelin sheets (see upper panel, Figure 3.11 on Page 96). This observation suggests that myelin sheet spreading might be driven by relaxation of the cortical tension at the sites of its appearance. Indeed, we found indications of changes in membrane tension during oligodendrocyte maturation (optical tweezer experiments performed by S. Nawaz, unpublished data).

It is widely accepted that the cortical actin network underlying the plasma membrane determines to a large extent the shape of animal cells. The actin cortex is under tension, and polarization of the cells often results from the local relaxation of this tension [345]. Cortex instability has been implicated in cell blebbing, commonly observed during cell spreading, division, migration or apoptosis [346, 347]. Blebs form at sites of cortex rapture or at sites of local detachment of membrane from the cortex (which is sometimes followed by cortex disassembly at the base of the bleb) [348]. Cortex instabilities can occur at multiple sites along the cell periphery, leading to multiple blebs, or can be a single event leading to a global polarization of the cell (as during polarization of the C.elegans embryo) [345].

Given that protrusion of the myelin sheets is indeed driven by actin cortex rapture, it is tempting to speculate that the observed strong expression of MBP in those regions ("half-moons", shown in Figure 3.9, on Page 93) mediates immediate compaction of the developing sheets in pOLs.

Taking into account aforementioned aspects, we suggest that myelin sheet expansion involves developmental downregulation of actin cytoskeleton. The resulting relaxation of cortex tension at the branching points of oligodendroglial processes en- 
ables protrusion of the myelin sheets, in which MBP promotes abrupt compaction. The proposed mechanism explains how myelin compaction and expansion could proceed in parallel, as observed in vivo (described in Section 1.1.1).

\subsection{New Membrane Incorporation into a Growing Myelin Sheet}

\subsubsection{Indications from Development}

To understand the mechanisms underlying myelin sheet growth and maintenance, we need to discern where exactly the new membrane is incorporated into it. To this end, we have examined the stages oligodendrocytes undergo during maturation. In the experiments described above (Section 4.2.1), we labeled the new developing sheets by immunostaing for MBP (shown in Figure 3.9 on Page 93). However, MBP has been shown to be translated locally at the myelin sheet assembly sites and transported to those sites in the form of mRNA [191]. Therefore, this strategy does not allow detection of plasma membrane components (lipids and proteins) arriving from the biosynthetic-secretory pathway. In order to mark the bulk myelin membrane incorporation, we used antibodies against the surface domain of PLP, a transmembrane protein of compact myelin, which follows the biosynthetic-secretory pathway [80].

At the "half-moon" stage of pOLs maturation, the plasma membrane PLP signal could be detected in between the MBP-positive domains. Later on, at the "sheets" stage, PLP staining was distributed over the cell surface (compared in Figure 3.8 on Page 91, middle and lower panels). This could result from lateral redistribution of PLP on the surface of pOLs. Another possibility is that the transport to the plasma membrane of the earlier and later PLP pools differ such that the earlier pools are delivered to the plasma membrane of the processes, while the later ones are targeted directly to the MBP-positive domains. To discriminate between these scenarios and address the question of new membrane incorporation into myelin sheet, we further analyzed vesicular trafficking in cultured primary oligodendrocytes. 


\subsubsection{Subcellular Localization of Membrane Extension Players}

Using immunocytochemistry we could detect vesicular trafficking and fusion machineries (microtubules, vesicles, SNAREs, Rabs, exocyst, etc.) in the processes of cultured oligodendrocytes. However, we did not find indications of enrichment of these components in any specific regions of the sheets. In fact, the myelin-like sheets seem to be depleted of the components examined throughout their developmental stages.

With respect to the localization of the transport tracks for intracellular trafficking in pOLs, we have shown that microtubules form dense bundles in oligodendroglial processes, but are excluded from the MBP-positive myelin-like sheets. These observations are in line with previous studies, describing localization of microtubules to cytoplasmic "veins" of oligodendrocytes [205, 325]. We found LAMP1-positive vesicles being restricted to the processes of pOLs, i.e. localized outside of the MBPpositive domains as well (see Figure 3.13 on Page 99).

The mediators of membrane fusion - the SNARE proteins - are known to reside predominantly in specific subcellular compartments [139]. This enabled us to choose the plasma membrane confined SNAREs (syntaxins 2, 3 and 4, SNAP-23) and the SNARE proteins restricted to the transport vesicles directed to plasma membrane (VAMP2/synaptobrevin) for immunocytochemical analysis. Specific localization of SNARE proteins in plasma membrane domains of pOLs has not been clarified yet, despite extensive work on myelin-associated SNAREs performed previously [156, 198, 199]. All the SNAREs we examined showed preferential localization to the processes and cell bodies of pOLs (see Figure 3.14 on Page 100 and Figure 3.15 on Page 101), which is in agreement with the subcellular localization of Stx3 as shown by D. Hoekstra [179]. However, his group made a different observation regarding Stx4, which they detected in the sheets [179].

Docking of transport vesicles to the myelin membrane can be regulated by Rab proteins (see [146] and Section 1.3.2). Distinct sets of Rab proteins are expressed in oligodendrocytes, with Rab3a being the most promising candidate for regulation of transport to plasma membrane [156]. In neurons, Rab3a is localized to synaptic vesicles and regulates neurotransmitter release (reviewed in [349]). We performed 
immunostainings of cultured primary oligodendrocytes for Rab3 and Rab3a proteins, which showed enrichment in the cell bodies and processes of pOLs (Figure 3.16). Consistent with our observations for Rab3/Rab3a localisation, Rab3a has previously been shown to colocalize with SNAP-29 in oligodendrocytes, which was detected mainly in the cell body and the major processes of pOLs [198].

Both endo- and exocytosis are regulated by the dynamics of membrane distribution of phosphatidylinositol 4,5-bisphosphate (PIP2) [158]. Here we show that PIP2 is predominantly localized to the processes and cell bodies of pOLs (see Figure 3.17 on Page 103), suggesting involvement of these oligodendroglial domains in membrane turnover. Interaction of PIP2 and MBP, which was previously shown by $\mathrm{S}$. Nawaz [318], could affect accessibility of some PIP2 epitopes to anti-PIP2 antibodies. However, implication of oligodendroglial processes and cell bodies in membrane turnover is further supported by the enrichment of proteins clathrin and caveolin in these regions (shown in Figure 3.18 on Page 104).

Recruitment of the transport vesicles to the areas of rapid membrane growth often employs an exocyst complex [143]. Engagement of exocyst in membrane tethering has been demonstrated in yeast and in polarized cells, such as epithelial cells, neurons and Schwann cells $[144,145,350]$. The Sec8 exocyst component is implicated in oligodendroglial morphological differentiation, but the data on its subcellular localization in pOLs is not consistent [180]. We show that the Sec8 protein is enriched in the cell bodies and processess of pOLs, confirming the involvement of these domains in exocytosis (see Figure 3.17 on Page 103).

\subsubsection{Hypothesis: Proximal Incorporation, Distal Flow}

Our observations on pOLs maturation and localization of vesicular trafficking/fusion machineries and intracellular pools of myelin proteins in pOLs (see Figure 3.19 on Page 105) indicate that intracellular trafficking takes place along the processes of oligodendrocytes.

These findings enable us to suggest novel directionality for myelin membrane growth: from the processes into the sheets domains (schematically shown in Figure 4.2). We speculate that the new myelin components, destined to form both 

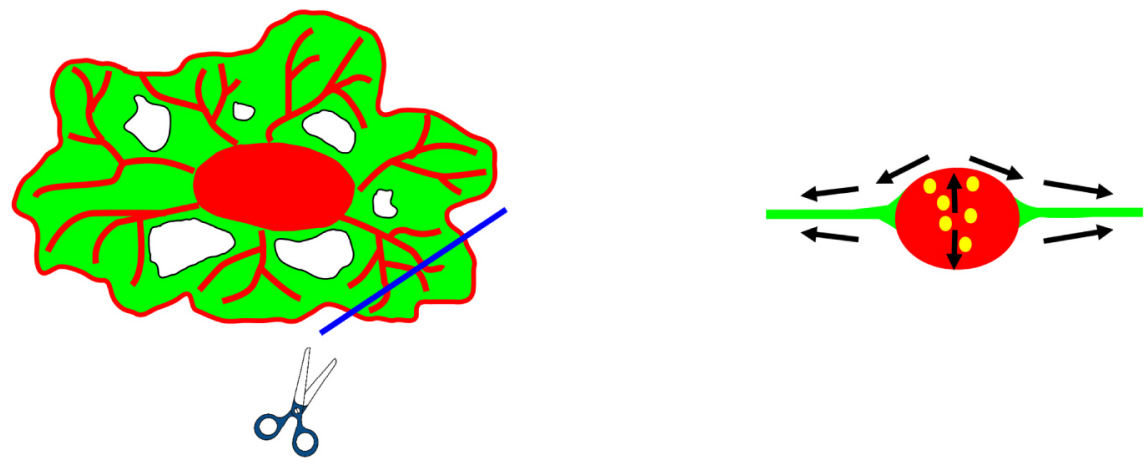

Figure 4.2: Schematic representation of the myelin growth hypothesis.

In a delineated primary oligodendrocyte, the processes and the cell body are highlighted in red, the sheets are in green. On the right, a putative cross-section through a cultured $\mathrm{pOL}$ is shown. In the center of the cross-section, a red oligodendroglial process harbors yellow vesicles, carrying new myelin components. The vesicles are incorporated into the plasma membrane of the process; the myelin sheet is extended by lateral flow of the plasma membrane.

compact and non-compact myelin domains, are produced, transported and incorporated into plasma membranes of pOLs in the processes, whereas the myelin-like sheets are generated by lateral spreading of the myelin components on plasma membrane. Lateral flow of the plasma membrane might be driven by incorporation of the new membrane, coming from the biosynthetic machinery.

Oligodendrocytes are polarized cells, like epithelial cells and neurons [156]. However, the proposed mechanism of membrane expansion in myelin-producing cells is radically different from the targeted or transcytotic delivery to apical and basolateral membranes, which was demonstrated for epithelial cells [175]. It is also distinguished from the mechanism of distal incorporation of membrane into the growth cone, invoking the cell body directed plasma membrane flow, currently thought to underlie axonal outgrowth [177]. Here, we suggest proximal membrane incorporation and distal flow for myelin biogenesis.

To support the hypothesis of proximal membrane incorporation in pOLs, visualization of the sites where the new membrane is added to the growing myelin sheet 
is required. For this, we have tagged compact and non-compact myelin proteins with a pH-sensitive GFP variant (pHluorin, [242]) at their extracellular domains. We are currently mapping exocytic events of pHluorin-MOG, pHluorin-MAG and pHluorin-TM4PLP to oligodendroglial cellular domains with total internal reflection fluorescence (TIRF) microscopy. Alternatively, to support the proximal membrane incorporation hypothesis, exocytosis could be monitored using plasma membrane lawns from pOLs [351]. Although we were successful in generating plasma membrane lawns from the oligodendroglial cell line Oli-neu and from primary microglial cultures, primary oligodendrocytes did not yield single-membrane lawns, possibly due to the compaction of their myelin-like sheets.

In addition, to assess the contribution of the biosynthetic-secretory machinery to the mobility of molecules in pOLs plasma membranes, we performed fluorescence recovery after photobleaching (FRAP) experiments. The FRAP data indicates that the new membrane incorporation into the growing plasma membrane indeed contributes to the mobility of the plasma membrane components tested (see Figure 3.22 on Page 109). To better resolve the flow component in the myelin-like membrane sheets, single particle tracking (SPT) experiments can be employed in future studies.

\subsection{Mechanisms of Myelin Domain Assembly}

Myelin is a polarized structure with distinct membrane heterogeneities, which can be broadly assigned as compact and non-compact myelin domains. During the characterization of the cellular model system for studying myelin, we have observed that primary oligodendrocytes are capable of establishing distinct plasma membrane domains in culture (see Section 4.1 and Figure 3.23 on Page 112). This ability is not unique to oligodendrocytes, as compartmentalization has been previously shown to be an intrinsic property of neurons, which extend distinct axons and dendrites in culture [352]. Unquestionably, in vivo the polarity, directionality and rate of pOLs membrane growth are modulated by the signals from the neurons [201]. Indeed, when cultured on coverslips without contact to neurons, pOLs extend their plasma membrane outgrowths radially. Nevertheless, the plasma membrane of oligodendrocytes displays distinct compartmentalization. Thus the ability to segregate plasma 
membrane and cellular constituents into distinct domains, which carry characteristics of either compact or non-compact myelin, appears to be an intrinsic property of oligodendroglial cells.

In contrast, our results (discussed in Section 4.3.3) suggest that during myelin sheet extension and/or maintenance, the newly produced components of compact and non-compact myelin are incorporated into the same plasma membrane domain. This apparent lack of discrimination in targeting of myelin components raises the question about the mechanisms of further segregation of myelin membrane into distinct domains.

Lateral heterogeneity after nondiscriminatory incorporation of components can be established by selective retention in specific membrane regions. Selective retention in plasma membrane can be mediated by cytoskeleton [229]. Thus, we first tested the possibility that the cytoskeleton imposes compartmentalization in pOLs plasma membranes. However, depolymerization of the actin cytoskeleton or microtubules did not result in redistribution of MAG and CNPase in the cell culture model (shown by J. Zimmermann, AG Simons).

Another candidate force for plasma membrane organization comes from differential interactions between its components, especially sphingolipids and cholesterol, which can lead to local membrane condensation and domain formation [221]. Sphingolipids and cholesterol are specifically enriched in myelin, making this lipid-driven mechanism likely to be involved by oligodendrocytes in the formation of its membrane domains. We performed a lipid analysis of membrane fractions isolated from pOLs, and were able to reveal quantitative differences in lipid composition of light and heavy pOLs membranes. Light membranes (compact-myelin-like) contained higher amounts of sphingolipids (Figure 3.25 on Page 115), than heavy membranes (non-compact-myelin-like). Furthermore, the acyl chains of the lipids in the light fractions were longer and more saturated than in the heavy fractions (Figure C.6 on Page 170). In addition, lighter fractions (compact-myelin-like), both isolated from pOLs and from myelin, were characterized by higher membrane order (shown with C-Laurdan, see Figure 3.26 on Page 116). We have also detected, that the compact myelin proteins were less soluble in detergent solubility tests (see Figure 3.24), confirming their association with detergent-resistant (more ordered) membrane do- 
mains. Taken together, these data imply lipid involvement in the assembly of myelin domains. To dissect the role of lipids in lateral organization of myelin membrane, we performed lipid depletion experiments.

\subsubsection{Role of Myelin Sphingolipids in Domain Formation}

Since sphingolipids were previously shown to promote formation of domains with higher lipid order (as shown in vitro in ternary mixtures with cholesterol and glycerophospholipids [220]), we probed galactosylceramide involvement in lateral organization of myelin membrane.

To reduce GalC levels in the cell culture model, we cultured pOLs in the presence of a specific inhibitor of ceramide synthase fumonisin B1 (FB1) [320]. Although FB1 has previously been reported to inhibit process outgrowth in cultured oliogodendroglial cells [211], we did not observe this phenotype. To model GalC depletion in vivo, we took advantage of mice deficient of ceramide synthase 2 (CerS2 KO), which show reduced levels of ceramide species with very long fatty acid residues, galactosylceramides and sulfatides in brain, and have compromised myelin maintenance [61]. Although mice lacking the enzyme UDP-galactose:ceramide galactosyltransferase (CGT) have been generated previously, we did not include them in this study due to possible compensation for GalC by glucosylcerebroside, reported in these mice [58].

To assess lateral organization in myelin membrane under sphingolipid depletion conditions, we measured membrane order with C-Laurdan fluorescent probe and lateral diffusion parameters with fluorescence correlation spectroscopy (FCS). CLaurdan allows estimation of lipid order in model and plasma membranes [296]. When integrated into the bilayer, the wavelength of the emission peak of C-Laurdan depends on degree of lipid packing. FCS is based on single-molecule sensitivity and analyzes the fluctuations in fluorescence intensity inside a small detection volume [293]. During the recent years, FCS has been successfully employed to study membrane dynamics both in model membranes and in living cells [226].

We have shown that both in cell culture and in vivo, sphingolipids are required for high lipid order and slow diffusion in the myelin membrane (see Figure 3.30 
on Page 121), as well as for lateral confinement of proteins in oligodendroglial plasma membrane (Figure 3.31 on Page 122). In oligodendrocytes, sphingolipids have been previously implemented in interactions of extracellular surfaces in apposing myelin leaflets and initiation of signalling processes, possibly involving MBP and cytoskeleton $[338,353,354]$. Yet, we did not observe redistribution of neither microtubules, nor microfilaments into the MBP-positive membrane sheets of oligodendrocytes (documented by J. Zimmermann, AG Simons). However, we detected that in addition to misorganization of myelin domains, as assessed from the distribution patterns of MAG and CNPase, inhibition of ceramide synthase with FB1 in culture induced changes in MBP immunostaining patterns. In fact, mislocalization of MAG or CNPase into the sheets of pOLs was associated with decreased MBP signal in the corresponding sheet areas (arrows, Figure 3.31 on Page 122). Therefore, further we sought to determine whether the observed effect of sphingolipid depletion on myelin membrane properties is direct or mediated by MBP.

\subsubsection{Regulation of MBP Levels by Sphingolipids}

We determined that sphingolipid depletion dramatically decreases MBP protein levels in cultured pOLs (see immunoblotting analysis in Figure 3.32 on Page 124). This observation is complemented by the reported decrease in MBP levels in the CerS2 $\mathrm{KO}$ myelin [61], and by the previously observed decrease in total MBP content in aggregating cultures of fetal rat brain [355]. Taken together, these data convincingly demonstrate that sphingolipids regulate the amounts of myelin basic protein in oligodendrocytes both in vivo and in culture. In addition, our results indicate that the regulatory effect of sphingolipids on MBP is rather exerted at the protein stability level, than at the transcriptional level (see Figure 3.33 on Page 125 and Figure 3.34 on Page 126).

Interestingly, the reverse regulatory effects cannot be excluded either. Thus, brain lipid composition of homozygote MBP-deficient mice (Shiverer) was reported to show a reduction in galactolipids [356]. Also in Shiverer heterozygotes, brain levels of galactocerebroside and sulfatide were decreased [329]. However, these data is difficult to interpret with respect to myelin composition, as mice lacking MBP have 
severe deficiency of the CNS myelin [75, 356]. Furthermore, since myelin is enriched in GalC, the observed reductions in Shiverer homo- and heterozygotes could result from the dysmyelination phenotype of these mice. However, mice carrying mutations in other myelin genes often display perturbations of their protein and/or lipid myelin composition [81]. Taken together, these data indicate that either incorporation or retention of myelin components in the myelin sheath is a cooperative process, in which proteins and lipids exert mutual regulation.

With respect to the depletion experiments, this implies that selective reduction in myelin levels of one specific component is unlikely to occur. Thus, in sphingolipid depletion experiments a more complex phenotype involving MBP depletion should be taken into account. Therefore, to verify the role of MBP in lateral organization of myelin, we further analyzed membranes of MBP-deficient oligodendrocytes.

\subsubsection{MBP Depletion in Cell Culture and In Vivo}

We prepared MBP-deficient oligodendroglial cultures and crude myelin samples from brains of Shiverer mice. Shiverer is an autosomal recessive mutation consisting of a deletion of the 3' end of the MBP gene which completely prevents production of mature mRNA and protein, and results in severe dysmyelination and a trembling behavior of animals [75]. Consistent with previous observations, we showed that the membranes prepared from Shiverer mice according to the crude myelin isolation protocol (detailed in Section 2.2.2) are denser than the WT myelin from the animals of the same age (P21), resembling myelin of younger animals, in which the myelination process is ongoing (P14) (see Figure 3.37 on Page 129 and [202, 329]). Despite severe dysmyelination of the CNS, Shiverer oligodendrocytes were shown to develop relatively normally in culture. However, a closer look at the distribution of non-compact myelin proteins (MAG and CNPase) in the plasma membrane of Shiverer pOLs revealed a dramatic loss of domain organization (see Figure 3.36 on Page 128), which was even more profound than the one induced by FB1 treatment. As in case of sphingolipid depletion, the cytoskeletal organization in most Shiverer oligodendrocytes was not notably compromised (shown in Figure 3.39 on Page 131). Both in the mature WT and in Shiverer pOLs, microfilaments had similar 
distribution and showed equally low abundance (with respect to the WT discussed in Section 4.2.2), although a small proportion of Shiverer pOLs with enlarged cell bodies and rudimentary sheets displayed less bundled distribution of microtubules, also observed previously [357].

Both in vivo and in culture, detected changes in Shiverer oligodendroglial membrane order, diffusion properties, lateral domain organization were even more severe than in the scenario observed after the sphingolipid depletion. Possibly, this is due to the absence of MBP expression in case of Shiverer [358], whereas MBP was dramatically underrepresented, but not absent in case of FB1 treatment or CerS2 deletion (see Figure 3.29 on Page 120 and [61]). Thus, both MBP deletion and sphingolipid depletion (which is associated with decrease in MBP amounts) not only induce disarrangement of proteins in oligodendroglial membranes (likely to be mediated by MBP), but also reduce lipid order in myelin membranes and increase diffusion rates in lipid vesicles, prepared from these membranes. Therefore, we further sought to investigate self-assembly properties of myelin lipids, both under normal and depletion conditions, in more detail.

\subsubsection{Lateral Segregation of Myelin Lipids in Model Membranes}

To examine the self-organization properties of myelin lipids, we performed in vitro experiments in giant unilamellar vesicles (GUVs). This in vitro assay is routinely used to study phase transitions in lipid mixtures of defined composition, aimed to model cellular membranes [220]. We observed lateral segregation, when lipids isolated from purified myelin and oligodendorglial cell membranes were combined in GUVs, but not in the GUVs formed from separate lipid extracts (see Figure 3.28 on Page 118).

The importance of compact myelin lipids for phase separation is supported by the observed lack of domain formation in GUVs prepared from combined lipid extracts from uncompacted myelin (Shiverer crude myelin) and oligodendroglial cell membranes. The role of sphingolipids in domain formation is further confirmed by observed homogeneity of GUVs, formed from lipids purified from CerS2 KO myelin and oligodendroglial cell membranes (see Figure 3.28 on Page 118). 
Together these data show that due to their enrichment in galactosylceramides, myelin lipids can self-organize and separate by the formation of different lipid phases, when observed in a protein-free in vitro system. The role of proteins in possible phase separation processes in biological membranes is currently a matter of controversy in the field [359]. It has been observed that in some model mixtures, interactions between the lipids dominate the clustering and phase behavior, whereas in other mixtures, protein-protein and protein-lipid interactions play a larger role [223]. While the protein effect on membrane phase organization depends on the specifics of the membrane studied, the role of proteins in myelin lipid self-organization needs to be assessed experimentally. To this end, it would be of interest to verify whether the GUVs composed of purified and combined myelin and plasma membrane would also exhibit domain formation, similarly to the lipid-only GUVs. Alternatively, phase separation can be probed in plasma membrane-derived vesicles GPMVs (giant plasma membrane vesicles) or PMS (plasma membrane spheres) [296].

In the next Section, we suggest possible implications of the observed self-organization of myelin lipids and MBP-dependent organization of the non-compact myelin proteins in the process of myelin membrane biogenesis.

\subsubsection{Phase Separation in the Context of Myelin Biogenesis}

Whereas phase separation has been shown to occur in model membranes in vitro [359], the situation in cellular membranes is still a subject of debate. Cellular membranes are crowded with proteins, which could exert distorting effects preventing self-organization of lipids within a membrane. In an exceptionally lipid-rich membrane however, as is the case with myelin, it can be argued that the scenario is closer to the model case than to other cellular membranes.

Myelin-producing cells have developed a remarkable capacity to generate a membrane with lipid contents as high as $70 \%$, and in addition, to allow only a limited number of proteins to reside in it [2]. Our findings indicate that restricting proteins from entering the myelin sheath (shown for MAG, CNPase, GFP-fusion proteins within the scope of this work, see Figure 3.23 on Page 112 and Figure C.4 on Page 168) requires $\mathrm{MBP}$. We speculate that $\mathrm{MBP}$ is involved in the formation of a diffu- 
sion barrier, which acts as a molecular sieve that is impermeable to most proteins, but not to lipids (unrestricted lipid diffusion was confirmed in FRAP experiments, see Figure C.7 on Page 171), thus being a precondition for the formation of a lipidrich membrane. We have shown this barrier to be regulated by sphingolipids, which are able to modulate cellular levels of MBP.

In addition to their regulatory role, sphingolipids themselves are important for lipid self-organization. Lipids of cellular membranes may form distinct phases, an ability that arises from the differential solubility of the lipids involved [217]. Glycosphingolipids (lipids composed of a ceramide backbone and a sugar headgroup), which are highly enriched in myelin [2], show self-associative behaviour in membranes [54]. These lipids have a tendency to form a hydrogen-bonded network with sphingomyelin and cholesterol in a phospholipid environment. Glycosphingolipids have much higher melting temperatures than glycerophospholipids, therefore the structural differences between these lipid classes allow sphingolipids to self-associate in the plane of the membrane [48].

In myelin-producing cells, the development of the diffusion barrier along with the high rate of sphingolipid synthesis may exploit the intrinsic potential of membrane lipids for phase separation. We suggest that these molecular mechanisms might underlie the formation of a highly ordered myelin membrane and ensure insulating properties of myelin, which facilitate rapid nerve conduction. 


\section{Chapter 5}

\section{Summary and Conclusions}

The work described in this thesis investigates mechanisms of myelin membrane extension and organization. Myelin formation and maintenance are indispensable for the proper functioning of the nervous system, as emphasised by the severe neurological deficits appearing in demyelinating diseases such as multiple sclerosis. So far the aforementioned aspects of myelin biogenesis have remained poorly understood due to the extreme compaction and nanometer thickness of myelin layers, which make these questions difficult to approach. To overcome these inherent difficulties, a cellular model representing two-dimensional "unrolled myelin" was chosen for this study. Although this work was mainly performed on primary cultures of myelin-producing cells, it also provides insights into the organization of myelin in vivo, as the major results from cell culture experiments were confirmed in CNS myelin preparations from mice.

To model the myelin sheath, we used cultured primary mouse oligodendrocytes. As evident from experimental data, in culture these cells develop flat myelin-like membrane sheets, which strikingly recapitulate the morphological and biochemical microcomposition of myelin in vivo. Cultured oligodendroglial cells establish two major subcellular compartments, that are cell body together with the processes and the flat membrane sheets, which carry properties of non-compact and compact myelin, respectively. The first question that was addressed in this study is how myelin membrane sheets extend, i.e. where the new myelin membrane is incorporated into the growing myelin sheet. Biochemical, immunocytochemical and live imaging data revealed preferred insertion of new membrane into the processes com- 
partment of growing oligodendrocytes. Based on this, we proposed a hypothesis of "proximal incorporation and distal flow". We suggest that the newly synthesized myelin membrane is transported along the processes of oligodendrocytes (corresponding to the non-compact myelin), where it is incorporated into the plasma membrane, followed by lateral diffusion to form the myelin sheets (corresponding to compact myelin).

These findings led us to the second question addressed in this study, namely the mechanisms of myelin membrane lateral organization. Surprisingly, primary oligodendrocytes develop distinct membrane domains without the requirement for neurons to wrap around, which allowed the assessment of myelin membrane organization in this cellular model. Since our data indicate that components belonging both to compact and non-compact myelin are initially incorporated into the same membrane domains (non-compact myelin), we asked how they are further sorted to form distinct membrane domains. Using biochemical and biophysical techniques in conjunction with fluorescence microscopy, we discovered the critical role of sphingolipids and myelin basic protein (MBP) in the lateral organization of myelin membrane, as well as the regulation of MBP amounts in primary oligodendrocytes by the sphingolipid dosage in these cells. Our findings indicate that in the myelin sheet, MBP is involved in the formation of a molecular barrier for proteins, allowing only a very limited set of protein species to enter the myelin sheath and thus enabling lipids to exert their potential for self-organization.

Taken together, the data presented in this thesis suggest that the myelin sheet is extended by membrane incorporation into the plasma membrane of oligodendroglial processes, but not into the sheet itself. Myelin expansion is accompanied by lateral segregation of myelin membrane components under the control of MBP and sphingolipids, which possibly employs lipid self-organization mechanisms. In addition, this work uncovered dramatic changes in the actin cytoskeleton during oligodendrocyte differentiation, raising the possibly that the protrusion of myelin sheets might be driven by local cortex relaxation. Furthermore, this study suggested size restrictions for the intracellular domains of the proteins residing in compact myelin, and established the basis for future elucidation of these aspects in myelin biogenesis. 


\section{Appendix A}

\section{Sequences}

\section{A.1 Sequences of Tags and Peptides}

Nucleotide and amino acid sequences of tags and peptides introduced by cloning into the expression constructs are listed in Table A.1.

Table A.1: Sequences of tags and peptides

\begin{tabular}{lll}
\hline Name & Nucleotide sequence & Amino acid sequence \\
\hline \hline HA tag & TACCCATACGATGTTCCAGATTACGCT & Y P Y D V P D Y A \\
myc-tag & GAGCAGAAACTCATCTCTGAAGAGGATCTG & E Q K L I S E E D L \\
tetracystein tag & TGTTGTCCTGGCTGTTGC & C C P G C C \\
thrombin cleavage site & CTGGTACCCCGAGGATCT & L V P R G S \\
\hline
\end{tabular}

\section{A.2 Sequences of Cloned Plasmids}

The cloning strategies for generation of the mammalian expression constructs used in this study are described below. The color scheme of the listed nucleotide sequences of the inserts is as follows:

- cDNA sequences of the myelin proteins are noted in black capital letters,

- red stands for restriction enzymes sequences,

- green indicates sequences of the tags,

- blue stands for thrombin protease cleavage sequence,

- sequences of glycine linkers are in yellow, 
- part of the Kozak sequence is in cyan color.

To generate the myc-thrombin-HA-MOG (clone No 62) construct (the sequence listed below in Table A.2), the inserts were amplified by PCR from the full-length MOG construct ([241]) and introduced in between ApaI and HindIII restriction enzymes sites of the multiple cloning site of pcDNA3.1(-) vector (Invitrogen, Carlsbad, CA 92008 USA).

Table A.2: Nucleotide sequence of myc-thrombin-HA-MOG (clone No 62)

10

GGGCCCGG

CCCTTCTCCT

CTGAAGAGGA

GAGGAGGAGG

CAGGGTATCC

CTGGGAAAAA

TTCACCTCTA

GCACAGAGCT

TGAGATTCTC

AGGCAGCAAT

CTCTCATCGC

TCCTGCAGCA

TTGATCCTCA

TTCTTGGACC

GACAGTTTCT

CTAGTAAGCT T
20

30

ATCCAAGATG

CCTCCTTCTC

TCTGGGAGGA

TACCCATAC

CATCCGGGCT

TGCCACGGGC

CCGAAATGGC

CCTGAAAGAG

AGATGAAGGA

GGAGTTGAAA

ACTTGTGCCT

CAGACTGAGA

CTTCCTGAGG

CCTGGTTGCC

TGAAGAgCTA

$\mathrm{T}$

GGAGGAAGCG

GATGTTCCAG

TTAGTTGGGG

ATGGAGGTGG

ACTATCAGTG

GTGCCCTGCT

TTGATCATCT
$40 \quad 50$

60

GCCTGTTTGT GGAGCTTCTC TTGGCCCAGC TGCTTCCTCT

CTCCAGTTGT CATGCAGCTA TGCAGAGCAG AAACTCATCT

GCCGCCTGGT

ACCCCGAGGA

TCTAGCGCT

ATTACGCTGG

ACAATTCAGA

GTGATAGGAC

ATGAAGCAGA

GCTGCCGTGC

CGCATCTCTC

GTTGGTACCG

TTCTCCCTTC

TCAAGAGTGG

AAGGACCAAG ATGCAGAGCA

AGCACCTGAA

TACCGGGGAC

AGGGAAAGGT

TACCCTTAGG

ATTCAGAACG

GGCTACACCT GCTTCTTCAG

AGACCACTCT

TACCAAGAAG

GTGGAAGATC CCTTCTATTG

GGTCAACCCC

GGTGTGCTGA

ACGATCCTCC TGCAGGTCTC

TGTAGGCCTT

GTATTCCTCT

GGAAAACTTC GTGCAGAAGT

AGAGAATCTC

CATCGGACTT

GGAAGATAAC

ACTGTTTGTT

ATTGTGCCTG

GCTACAACTG

GCTGCACCGA

AGACTGGCAG

AGAAACCCCT TTggtgctTG

TTGTCCTGGC TGTTGCTGAA

pHluorin-MOG (clone No 69) was generated by replacing the thrombin protease cleavage site in clone No 62 (flanked with NotI and AfeI restriction sites, see the sequence above) with pHluorin sequence, amplified from pHluorin-synaptobrevin plasmid (see Table 2.2, a kind gift from Prof. G. Miesenböck, University of Oxford, Oxford, UK) with 16934 and 16935 primers (see Table A.3).

To produce pHluorin-thrombin-MOG (clone No 82), the MOG cDNA region was amplified from the full-length MOG construct ([241]) with 17052 and 17053 primers (see Table A.3) containing the thrombin protease cleavage site. The PCR-amplified region was introduced in between AfeI and HindIII restriction sites of pHluorin-MOG (clone No 69). The insert sequence of pHluorin-thrombin-MOG (clone No 82) is listed in Table A.4. 
pHluorin-MAG (clone No 96) was generated by replacing MOG sequence in clone No 69 (flanked with AfeI and HindIII restriction sites, see the sequence above) with thrombin-HA-MAG sequence, amplified from clone No 49 (generated by replacing the GFP sequence with myc-thrombin-HA oligonucleotide in $\mathrm{p} \beta$-actin-GFP-S-MAG construct [115]) with 19198 and 19199 primers (see Table A.3).

To produce pHluorin-PLPTM4 (clone No 100) construct, the sequence of the 4 th transmembrane domain of the Mus musculus proteolipid protein was PCR-amplified with 19196 and 19228 primers from the PLP-myc-EGFP construct [201] and introduced into clone No 82 with EcoRI and HindIII restriction enzymes instead of the MOG sequence.

Primers, used for cloning of the constructs described above, are listed in Table A.3.

Table A.3: List of primers used for cloning

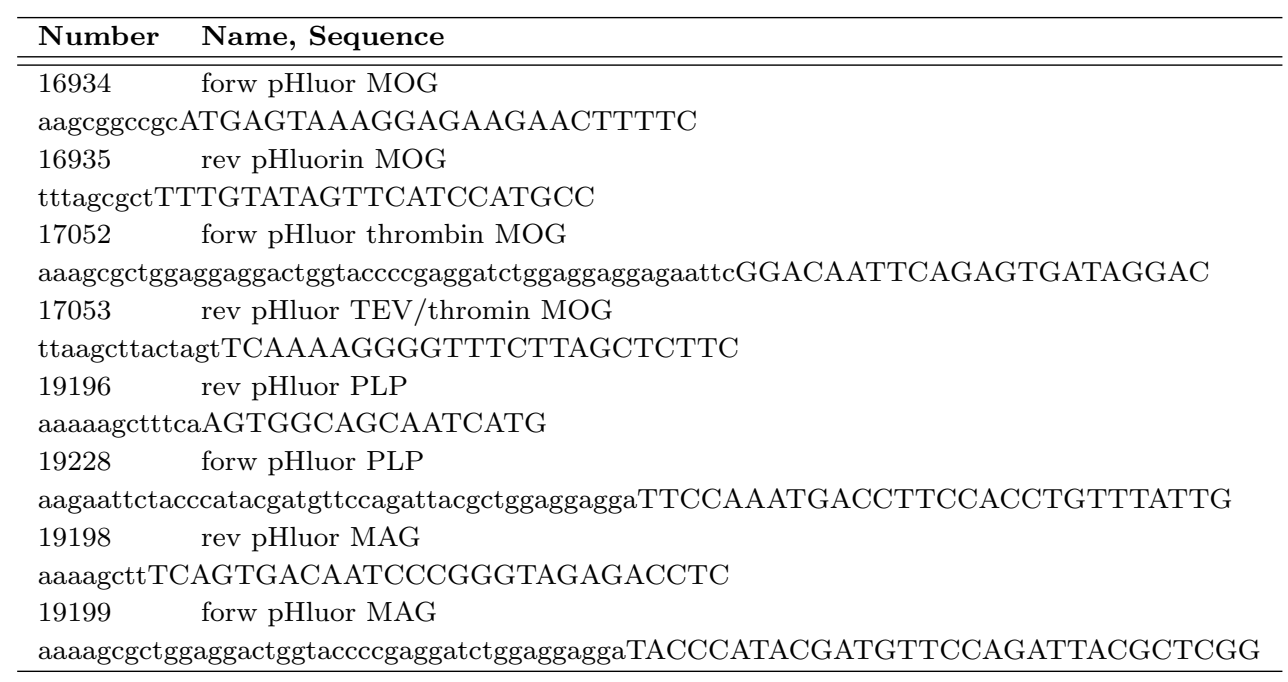

Here, the parts of the primer sequences, complementary to the amplified regions of the myelin proteins are indicated in capital letters, whereas sequences coding for the additional restriction sites, tags, etc. are in lower-case letters. 
Table A.4: Nucleotide sequence of pHluorin-thrombin-MOG (clone No 82)

10

GGGCCCGG CCCTTCTCCT

CTGAAGAGGA

ctggagttgt

tcagtggaga

ctactggaaa

aatgctttc

ccgaaggtta

gtgctgaagt

attttaaaga

aggtgtacat

acaacattga

gcgatgggcc

aagatcccaa

ttacacatgg

CT

TAGTTGGGGA

TGGAGGTGGG

AGGACCAAGA

CTATCAGTGA

GCTACACCTG

TGGAAGATCC

CGATCCTCCT

GAAAACTTCG

TGCCCTGCTG

TGATCATCTG

GAAACCCCTT
20

ATCCAAGATG

CCTCCTTCTC

TCTGGGAGGA

cccaattctt

gggtgaaggt

actacctgtt

aagataccca

tgtacaggaa

caagtttgaa

agatggaaac

catggcagac

agatggaggc

cgtccttta

cgaaaagaga

catggatgaa

GAATTCGGA

TGAAGCAGAG

TTGGTACCGT

TGCAGAGCAA

GGGAAAGGTT

CTTCTTCAGA

CTTCTATTGG

GCAGGTCTCT

TGCAGAAGTA

GAAGATAACA

CTACAACTGG

TTGAACTAGT
30

40

50

60

GCCTGTTTGT

CTCCAGTTGT

AGCG

GGAGCTTCTC

TTGGCCCAGC

TGCAGAGCAG

taaaggagaa

taatgggcac

tacccttaaa

tactttaact

ctttttcaag

tgacgggaac

aatcgagtta

atacaactat

agctaacttc

tcaacaaaat

tacaacttct

gtttgtaaca

CTGGTA

AGGGTATCCC

TGGGAAAAAT

TCACCTCTAC

CACAGAGCTC

GAGATTCTCA

GGCAGCAATG

TCTCATCGCA

CCTGCAGCAC

TGATCCTCAC

TCTTGGACCC

ACAGTTTCTT
TGCTTCCTCT

AAACTCATCT

gaactttca

aaattttctg

tttatttgca

tatggtgttc

agtgccatgc

tacaagacac

aaaggtattg

aacgatcacc

aaaattagac

actccaattg

actctttcga

gctgctggga

CCCCGAGGAT

ATCCGGGCTT

GCCACGGGCA

CGAAATGGCA

CTGAAAGAGA

GATGAAGGAG

GAGTTGAAAG

CTTGTGCCTA

AGACTGAGAG

TTCCTGAGGG

CTGGTTGCCT

GAAGAgCTAA

Here, pHluorin sequence is in lower-case letters, an extra nucleotide introduced to avoid the frame shift is also noted in a lower-case letter (yellow letter "a"). 


\section{Appendix B}

\section{Source Code}

\section{B.1 ImageJ Macros}

\section{B.1.1 Calculation of the Correlation Coefficient Between the Two Channels}

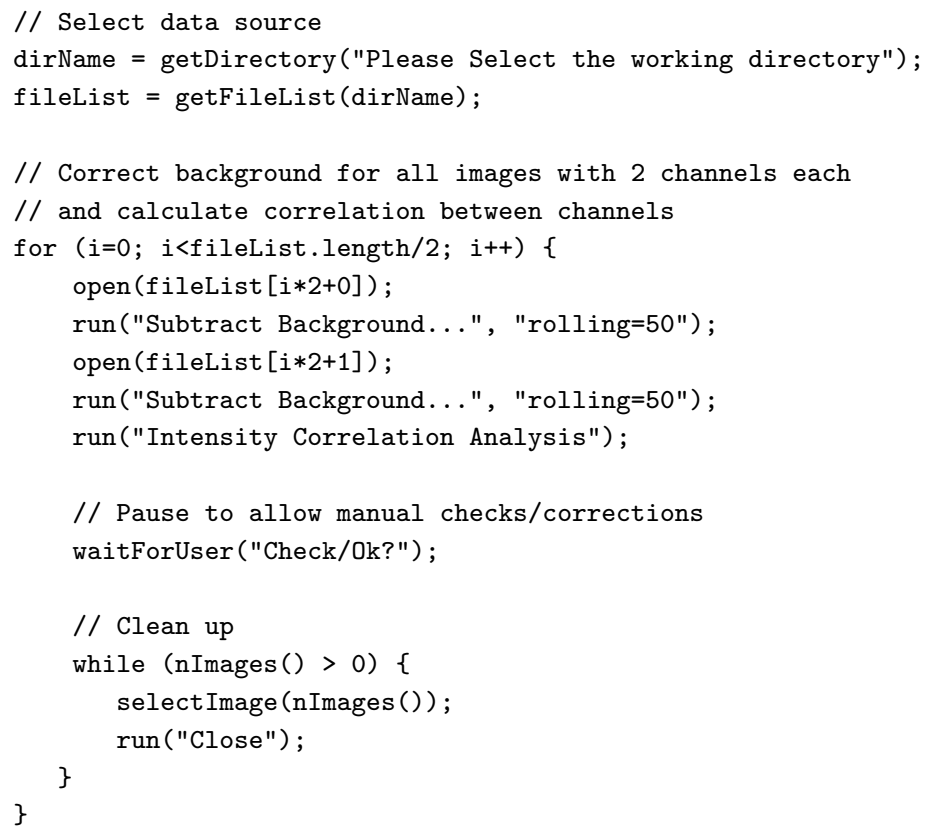




\section{B.1.2 Measuring Total Fluorescence Intensities}

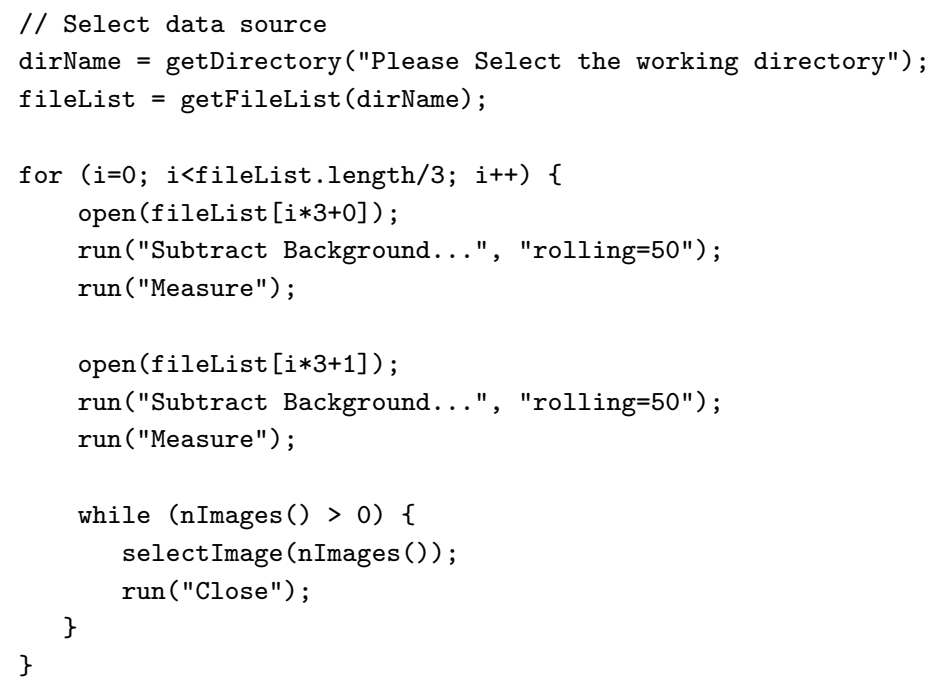

\section{B.1.3 Measuring ROI Fluorescence Intensities}

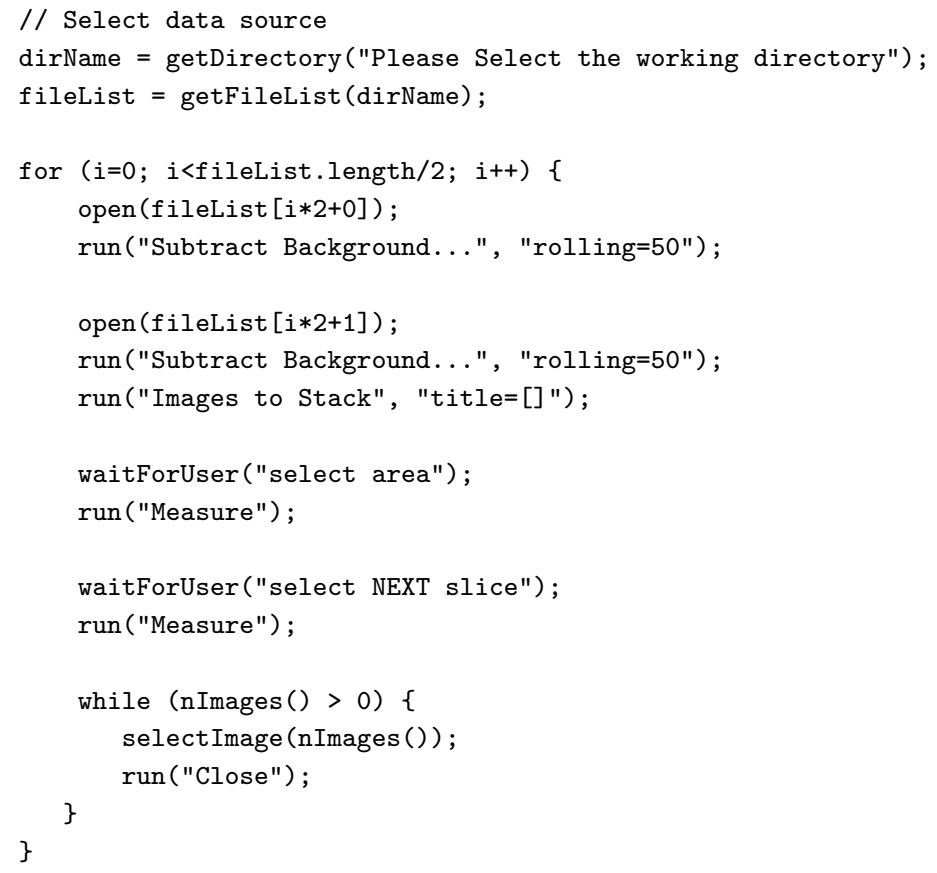




\section{B.1.4 Image Processing, Cropping, Channel Merge}

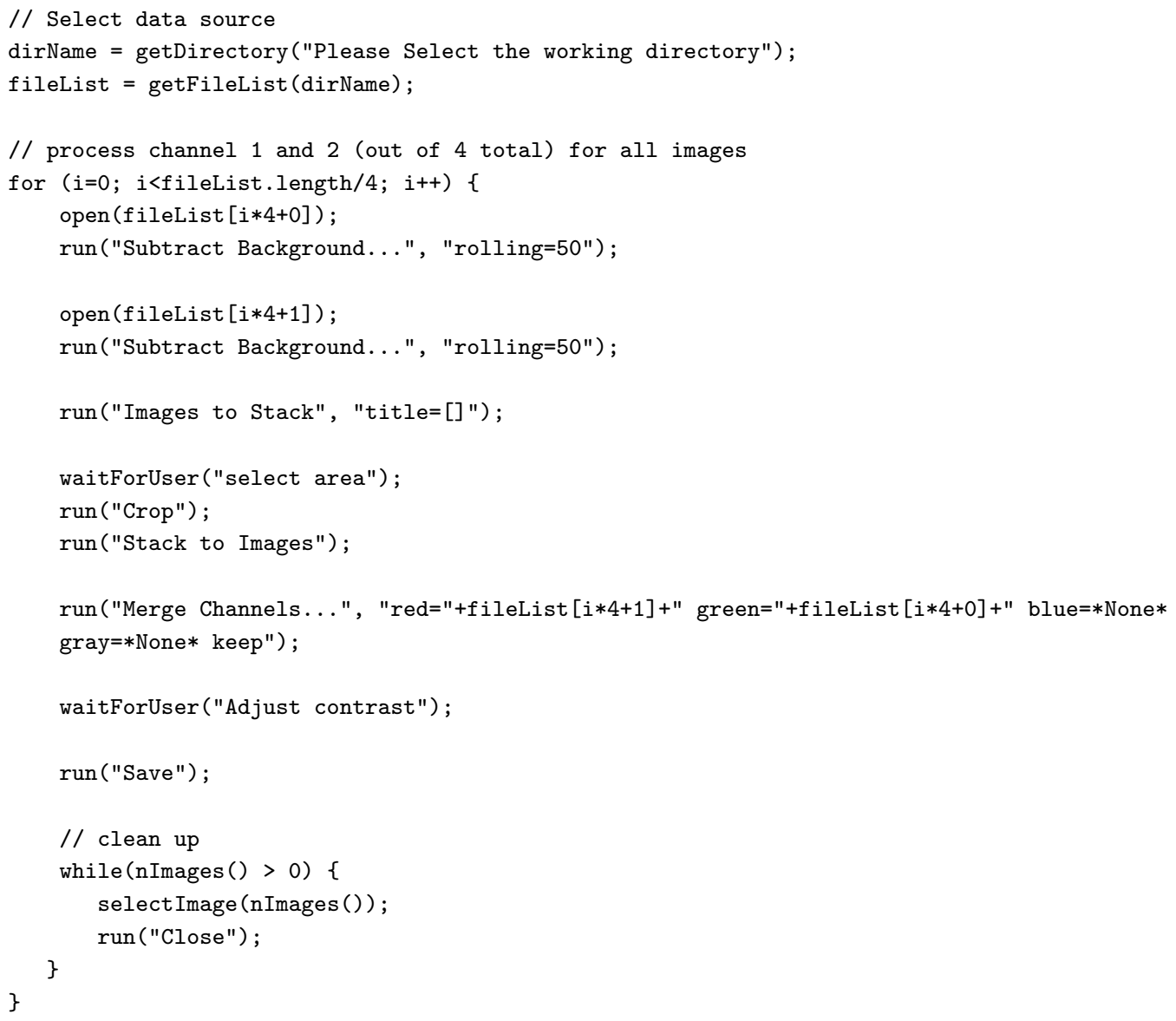




\section{Appendix C}

\section{Supplementary Figures}
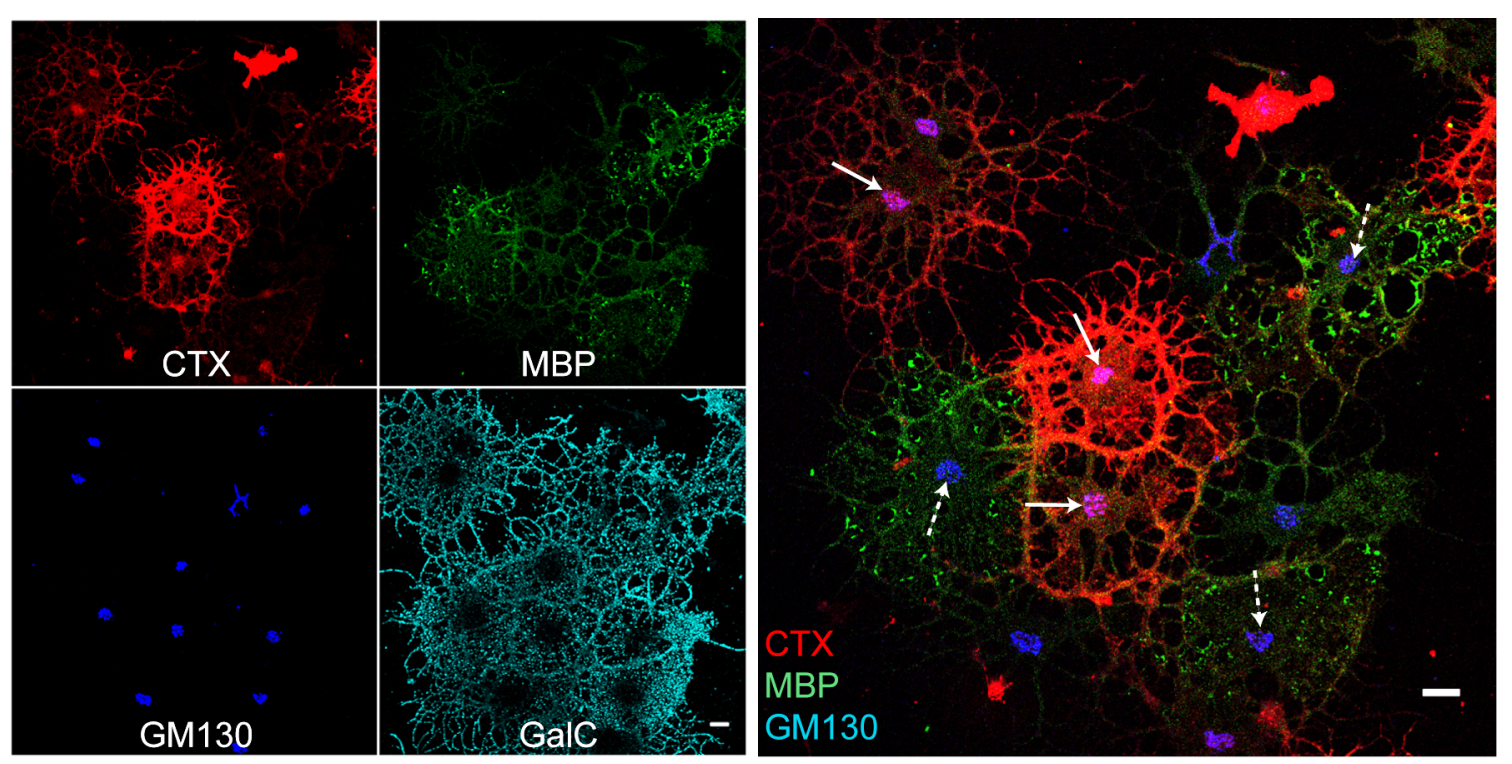

Figure C.1: Endocytosis in developing oligodendrocytes: Cholera toxin (CTX) uptake. Mouse primary oligodendrocytes, maintained in cultures for 3 days after shake, were incubated with Cholera toxin subunit B (CTX, red) coupled to Alexa Fluor $\AA 555$ (Invitrogen $\mathrm{GmbH}$, Darmstadt, Germany) to allow binding to its plasma membrane receptor GM1. Internalization of CTX was carried out for $30 \mathrm{~min}$ at $37^{\circ} \mathrm{C}$. The cells were fixed, permeabilized, stained for MBP (green), GM130 (blue), O1 (cyan) and imaged with a confocal microscope. Overlay image demonstrates higher enrichment of internalized CTX signal in the Golgi complex (GM130 staining, blue) in MBP-negative younger cells (white arrows), than in more mature MBP-positive cells (dashed arrows). Scale bar, $10 \mu \mathrm{m}$. 


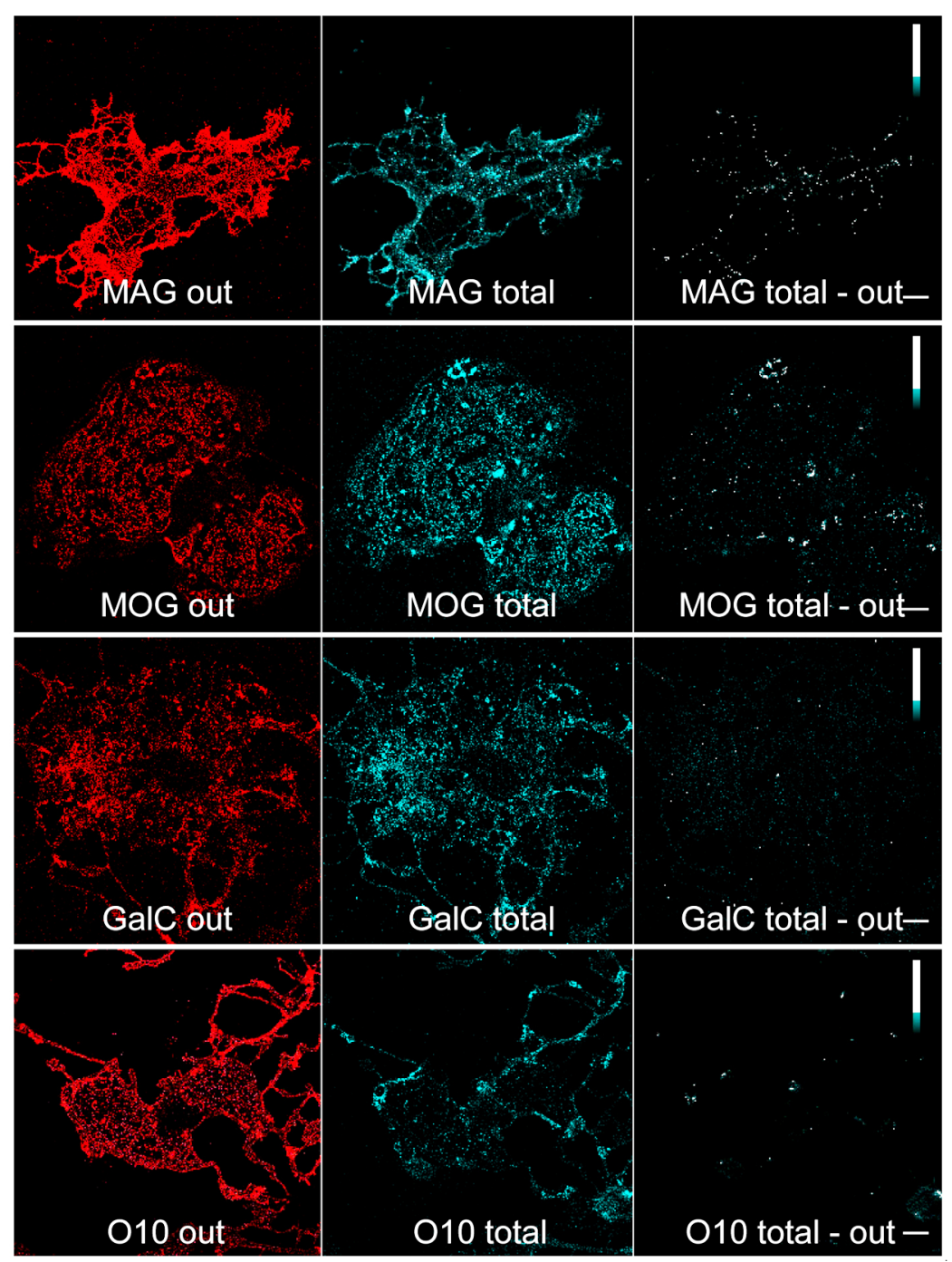

Figure C.2: Endocytosis of MAG, MOG, GalC and PLP epitopes.

Live cultured oligodendrocytes ( 5 - 6 days after shake) were incubated with primary antibodies against surface epitopes of MAG, MOG, GalC or O10(PLP) for $10 \mathrm{~min}$ at $4^{\circ} \mathrm{C}$ to allow specific binding. Unbound immunoglobulins were washed off; the epitopes were allowed to internalize for 30 or 120 min (only a 30 min time-point is shown). After internalization, the cells were fixed, their surfaces were stained with appropriate secondary antibodies (red), followed by permeabilization and detection of the total pool of primary antibodies (internalized and remaining surface epitopes) with secondary antibodies, coupled to a different fluorophore (cyan). The right panel shows the endocytosed pools; these images were calculated by subtracting the surface signals from the total signals. Scale bar, $10 \mu \mathrm{m}$. 
a Endocytosis of myelin epitopes, $30 \mathrm{~min}$

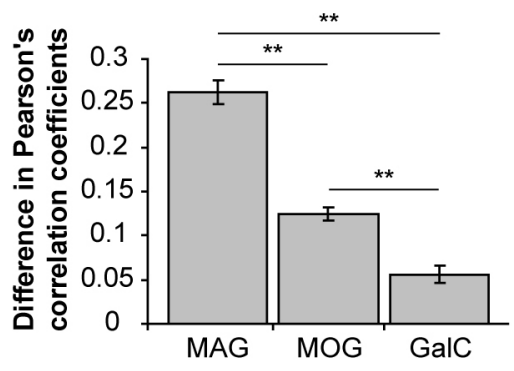

b Dynamics of myelin epitopes endocytosis

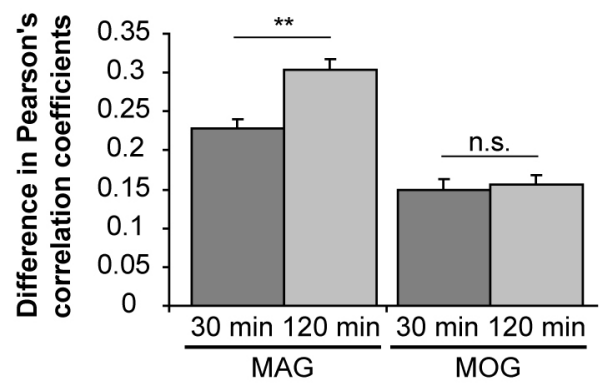

Figure C.3: Quantitative analysis of endocytosis of MAG, MOG and GalC.

The endocytosis assays were performed as described for Figure C.2.

(a) To compare endocytosis rates for MAG, MOG and GalC, Pearson's correlation coefficients were calculated between the channels corresponding to surface stainings and to total signals after $0 \mathrm{~min}$ and after $30 \mathrm{~min}$ of internalization. The $30 \mathrm{~min}$ values were subtracted from the 0 min values to obtain decrease in colocalization of the surface and total epitopes. Higher endocytic activity results in larger difference in colocalization. The differences (relative endocytic activity), calculated for MAG, MOG and GalC were analyzed with ANOVA, followed by pairwise multiple comparison (Tukey test); ** denotes significantly different pairs, $\mathrm{P}<0.01, \mathrm{n}=38-42$.

(b) To analyze the dynamics of MAG and MOG endocytosis, 30 min and 120 min internalization time-points were compared. Decrease in colocalization of MAG surface and total signals was significantly higher after $120 \mathrm{~min}$, than after $30 \mathrm{~min}$ (t-test, ${ }^{* *} \mathrm{P}<0.01, \mathrm{n}=40$ - 42), indicating progression of endocytosis. Difference in colocalization of MOG surface and total signals after 30 and 120 min of uptake was not significant. 

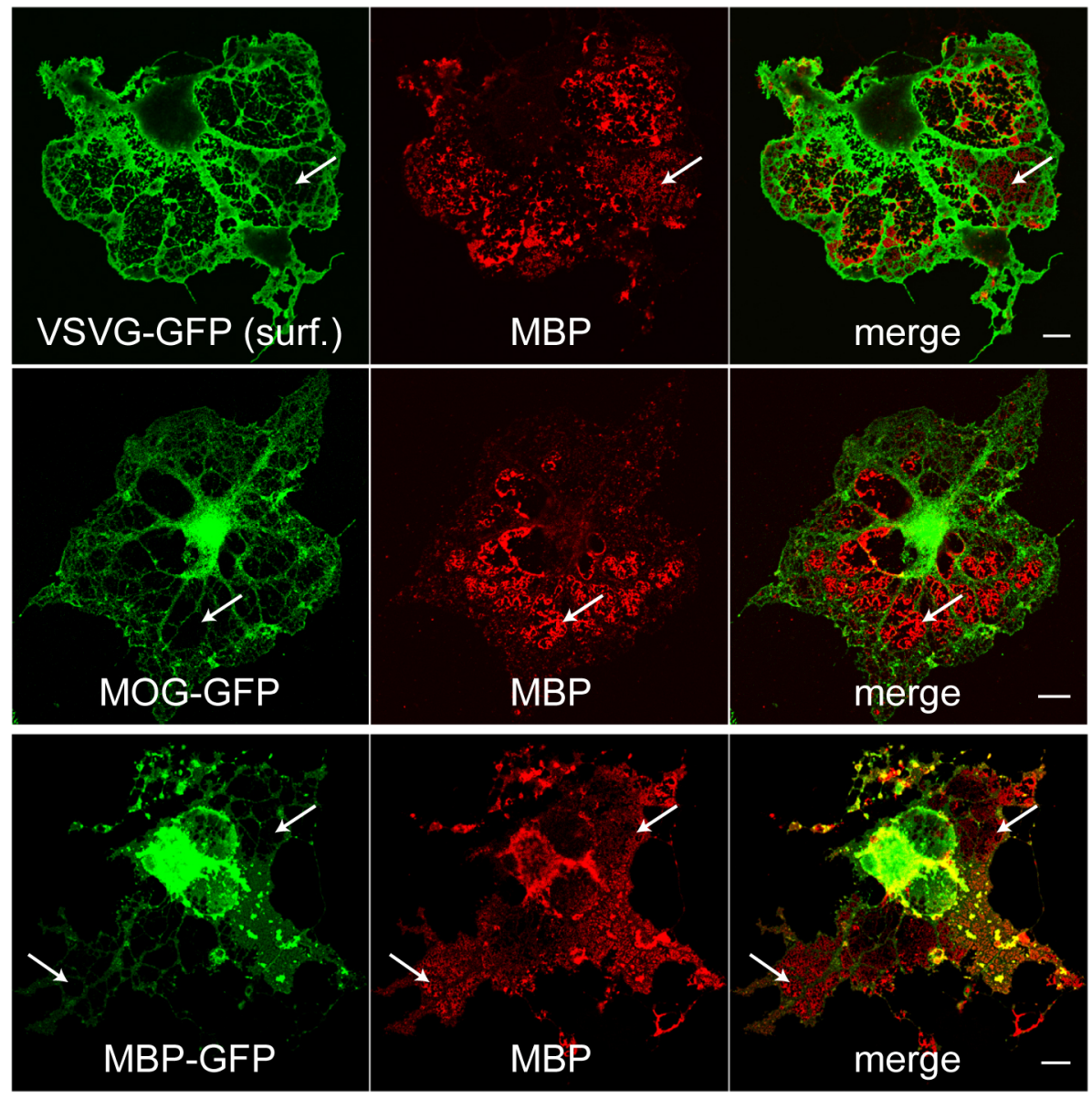

Figure C.4: Subcellular localization of overexpressed VSVG-YFP, MOG-GFP and MBPGFP in pOLs.

Cultured primary oligodendrocytes were infected with SFV carrying VSVG-YFP or MBPGFP or transfected with MOG-GFP, immunostained and imaged with confocal microscope. Immunolabeling for surface epitopes of VSVG (green), fluorescent signals of MOG-GFP (green) and MBP-GFP (green) are shown. Endogenous MBP was immunostained to resolve the MBP-positive membrane domains (red). Note that proteins tagged on their intracellular domains with fluorescent proteins do not localize to MBP-positive membrane sheets (white arrows). Furthermore, MBP-GFP overexpression is toxic to cells and leads to aggregation of MBP-GFP in cell bodies of pOLs (lower panel). Scale bar, $10 \mu \mathrm{m}$. 


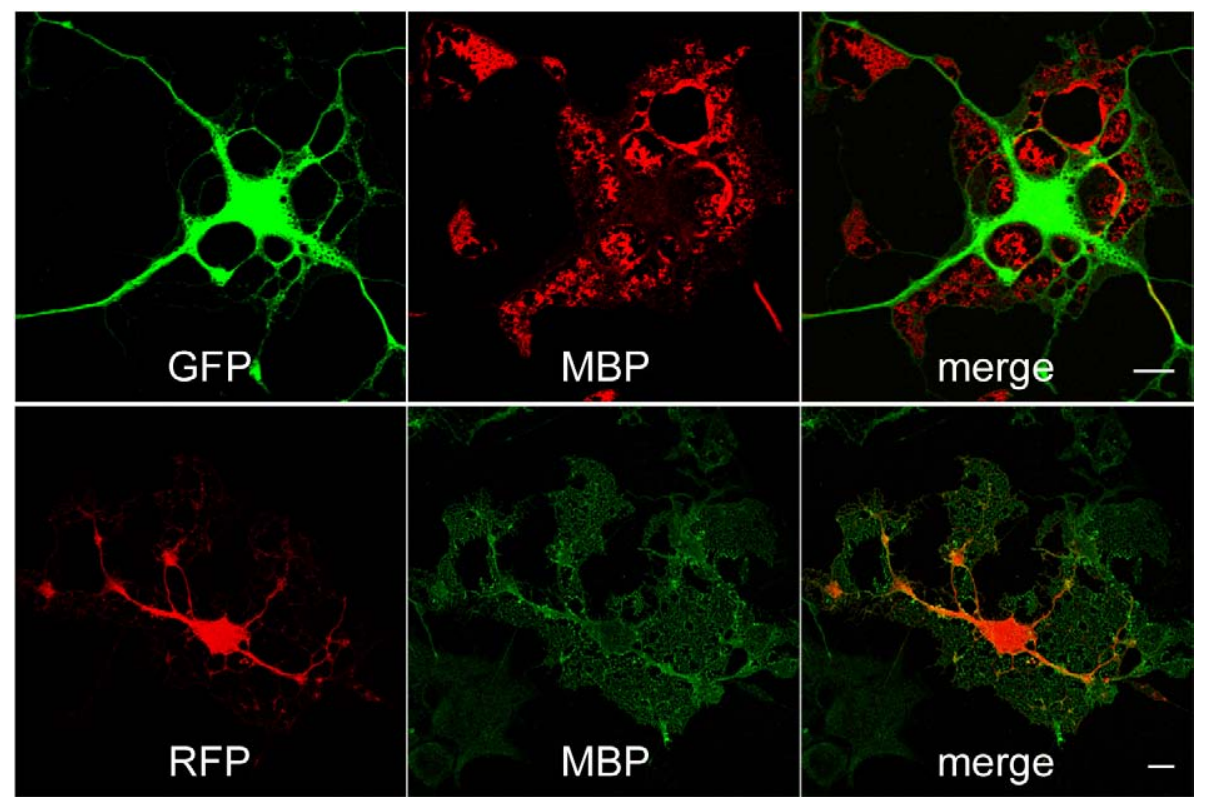

Figure C.5: Subcellular localization of overexpressed GFP and RFP in pOLs.

Cultured primary oligodendrocytes were transfected with mammalian expression constructs carrying GFP or RFP, immunostained and imaged with confocal microscope. Immunostaining for MBP shows MBP-positive membrane domains. Note very weak fluorescence of GFP and RFP in MBP-positive sheets. Scale bar, $10 \mu \mathrm{m}$. 
a

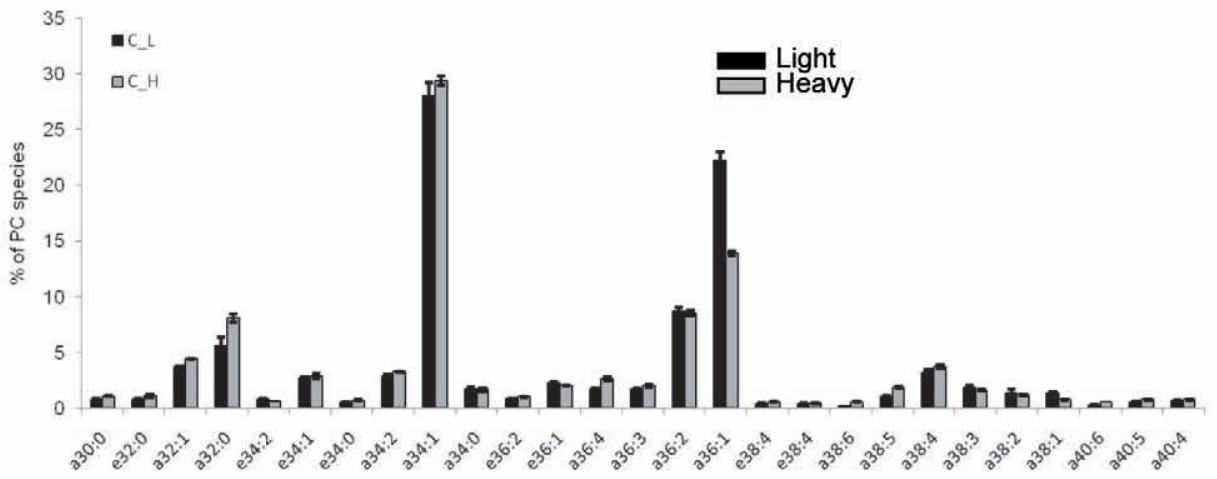

b

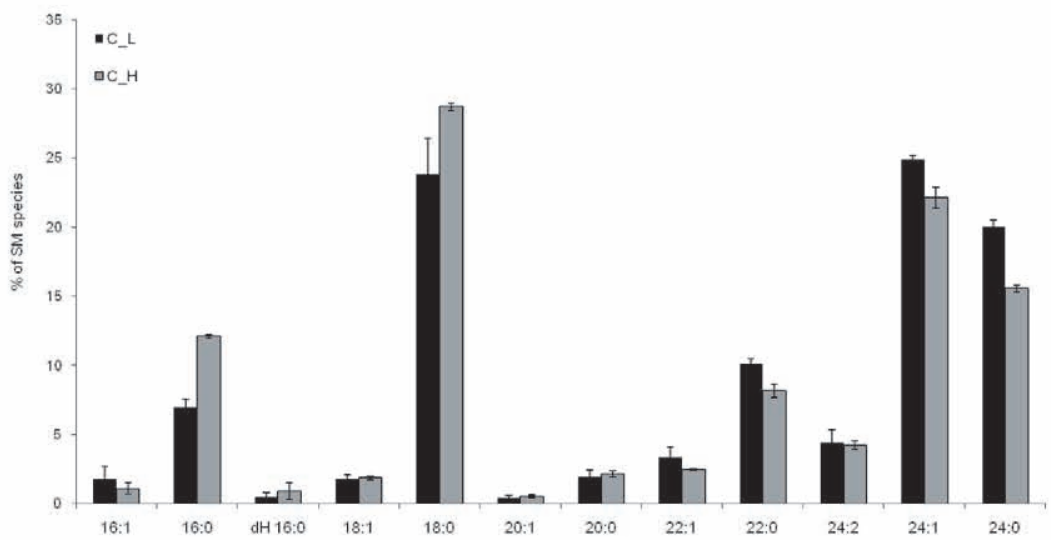

C

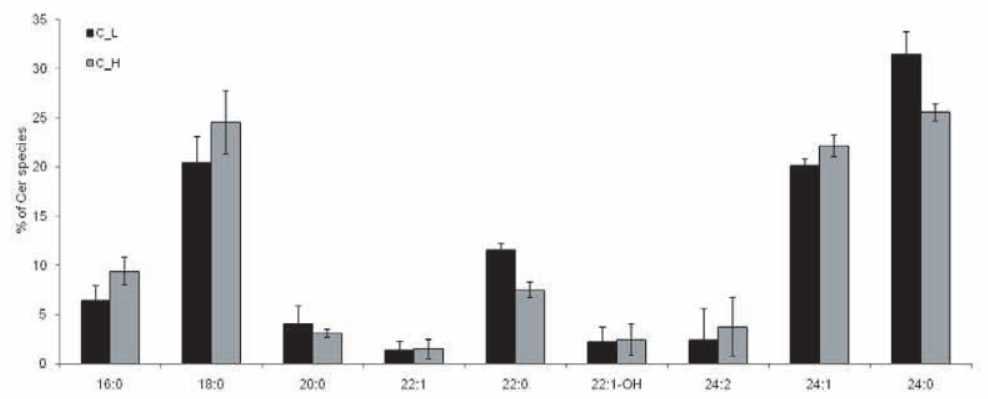

d

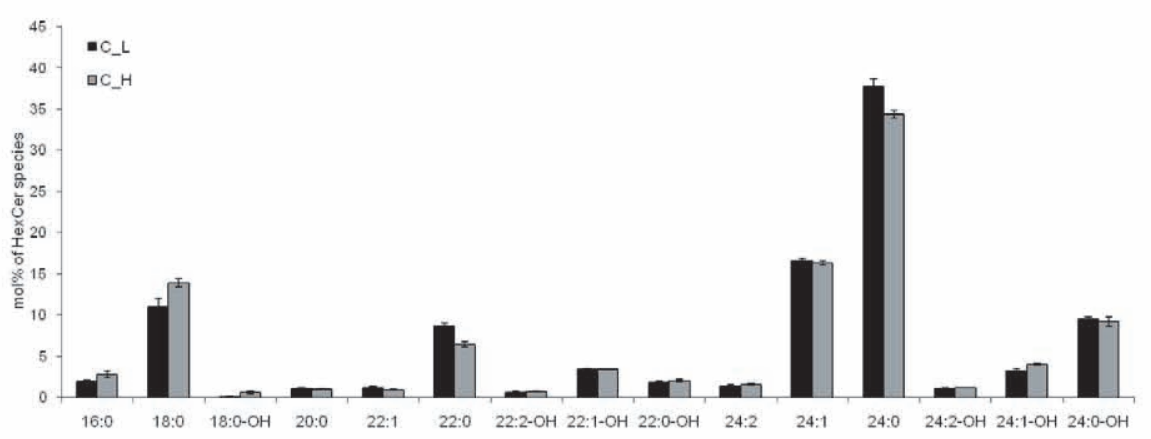

Figure C.6: Quantitative analysis of lipid molecular species in membrane subfractions from cultured primary oligodendrocytes.

Black and gray bars correspond to light and heavy membrane fractions respectively. Bars represent means $\pm S D$ from three independent experiments. Measurements are courtesy of B. Brügger. 
a

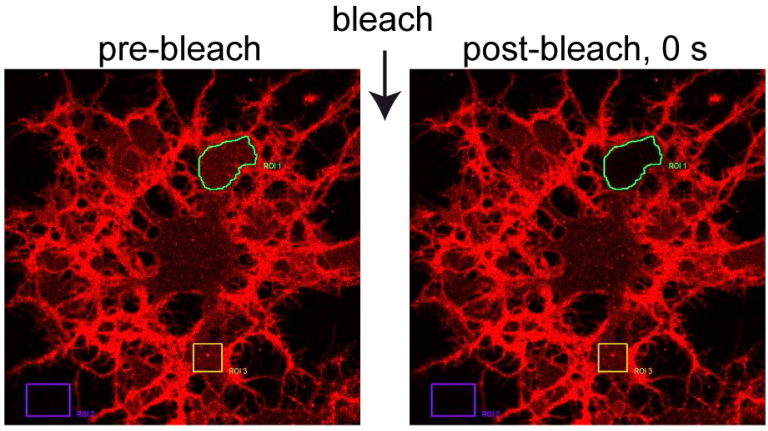

post-bleach, $15 \mathrm{~s}$

post-bleach, $50 \mathrm{~s}$

b

FRAP Cell Mask Orange, whole sheet

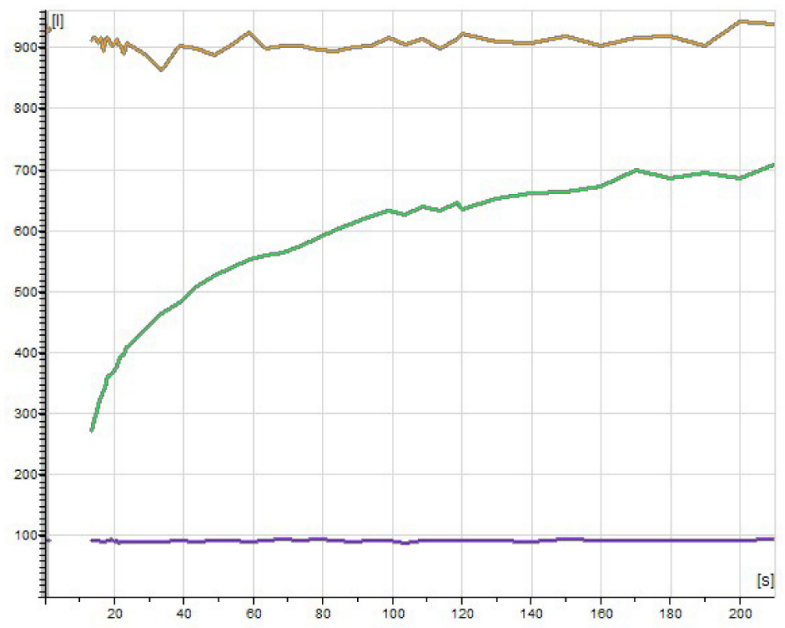

Figure C.7: FRAP analysis: free lipid diffusion in the myelin sheet.

(a) Pre-bleach and post-bleach confocal images of a primary oligodendrocyte, stained with Cell Mask Orange fluorescent lipid dye (Invitrogen $\mathrm{GmbH}$, Darmstadt, Germany) on the surface. In this experiment, the whole membrane sheets of pOLs were bleached to test whether lateral diffusion from the adjacent processes is permitted. Scale bar, $10 \mu \mathrm{m}$. (b) Graph shows a recovery curve of fluorescent intensities in the bleached region (green line). Violet line indicates background fluorescence, yellow line shows fluorescence in the unbleached region. 


\section{Appendix D}

\section{List of Symbols and Abbreviations}

$\begin{array}{ll}\text { AFM } & \text { Atomic force microscopy } \\ \text { ANOVA } & \text { One way analysis of variance } \\ \text { APC } & \text { Antigen presenting cell } \\ \text { APP } & \text { Amyloid precursor protein } \\ \text { APS } & \text { Ammonium persulfate } \\ \text { BCA } & \text { Bicinchoninic acid } \\ \text { BSA } & \text { Bovine serum albumin } \\ \text { C-Laurdan } & \text { 6-dodecanoyl-2-methylcarboxymethylaminonaphthalene } \\ \text { CHAPS } & 3 \text {-[3-(Cholamidopropyl)dimethylammonio]-1-proanesulfonate } \\ \text { CNPase } & 2 \text { ','-cyclic-nucleotide 3'-phosphodiesterase } \\ \text { CNS } & \text { Central nervous system } \\ \text { COPI, COPII } & \text { Cytosolic coat proteins I and II } \\ \text { CSF } & \text { Cerebrospinal fluid } \\ \text { CTX } & \text { Cholera toxin } \\ \text { DNA } & \text { Deoxyribonucleic acid } \\ \text { DOPC } & \text { Dioleoylphosphatadiylcholine } \\ \text { EAE } & \text { Experimental autoimmune encephalomyelitis } \\ \text { EDTA } & \text { Ethylenediaminetetraacetic acid } \\ \text { EGF } & \text { Epidermal grotwh factor } \\ \text { EGFR } & \text { Epidermal grotwh factor receptor } \\ \text { ER } & \text { Endoplasmic reticulum } \\ \text { EtBr } & \text { Ethidium bromide } \\ \text { FB1 } & \text { Fumonisin B1 } \\ \text { FP } & \text { Fluorescent protein } \\ \text { FRAP } & \text { Fluorescence recovery after photobleaching } \\ \text { GalC } & \text { Galactosylceramide } \\ \text { GFP } & \text { Green fluorescent protein } \\ & \\ & \end{array}$


GM130

GPI

GTP

GTPase

GUV

$\mathrm{h}$

HA

HEPES

HRP

$\mathrm{kDa}$

LAMP1

Laurdan

MAG

MBP

$\min$

MOG

MOPS

NSF

P0, P14, P21, P60

PAGE

PBS

PCR

PFA

$\mathrm{PH}$

pHluorin

PI

PIP2, PI(4,5)P2

$\mathrm{PKC}$

PLC

PLD

PLL

PLP

pOLs

PS

Rab

RFP

RNA

RNase

ROI
Golgi matrix protein, $130 \mathrm{kDa}$

Glycosyl phosphatidylinositol

Guanosine triphosphate

Guanine triphosphatases

Giant unilamellar vesicle

Hour(s)

Hemagglutinin tag

4-(2-hydroxyethyl)-1-piperazineethanesulfonic acid

Horse radish peroxidase

Kilodalton

Lysosomal-associated membrane protein 1

6-dodecanoyl-2-dimethylaminonaphthalene

Myelin-associated glycoprotein

Myelin basic protein

Minute(s)

Myelin oligodendrocyte glycoprotein

3-(N-Morpholino)propanesulfonic acid

N-ethylmaleimide sensitive factor

Postnatal day $0,14,21$ or 60

Polyacrylamide gel electrophoresis

Phosphate buffered saline

Polymerase chain reaction

Paraformaldehyde

Pleckstrin homology

$\mathrm{pH}$-sensitive green fluorescent protein

Phosphatidylinositol

Phosphatidylinositol 4,5-bisphosphate

Protein kinase $\mathrm{C}$

Phospholipase C

Phospholipase D

Poly-L-lysine

Proteolipid protein

Primary oligodendrocytes

Phosphatidylserine

Ras like protein in brain

Red fluorescent protein

Ribonucleic acid

Ribonuclease

Region of interest (microscopy) 
RT

$\mathrm{S}$

$\mathrm{SD}$

SDS

SEM

SFV

SNAP-25

SNAP-29

SNAPS

SNARE

SPT

Stx

Taq DNA polymerase

TCA

TEMED

TGN

TIRF

VAMP

VLP

VSVG

YFP
Room Temperature

Second(s)

Standard deviation

Sodium dodecyl sulphate

Standard error of the mean

Semliki forest virus

Synaptosome-associated protein, $25 \mathrm{kDa}$

Synaptosome-associated protein, $29 \mathrm{kDa}$

Soluble NSF attachment proteins

Soluble NSF attachment protein receptor

Single particle tracking

Syntaxin

DNA polymerase from Thermus aquaticus

Trichloroacetic acid

N'N'N'-tetramethylethylene diamine

Trans-Golgi network

Total internal reflection fluorescence

Vesicle-associated membrane protein

Virus like particle

Vesicular stomatitis virus glycoprotein

Yellow fluorescent protein 
Fractions of RNA or DNA, or functions exercised by preparations of RNA and DNA are designated as follows:

$$
\begin{array}{ll}
\text { cDNA } & \text { Complementary DNA } \\
\text { mRNA } & \text { Messenger RNA } \\
\text { rRNA } & \text { Ribosomal RNA } \\
\text { siRNA } & \text { Small interfering RNA } \\
\text { tRNA } & \text { Transfer RNA }
\end{array}
$$

By convention, single strands of DNA and RNA sequences were written in 5'- to 3'-direction [360]. For the representation of oligonucleotides and polynucleotides the oneletter system was used. Indicated letters refer to the corresponding purine or pyrimidine bases:
A Adenine
C Cytosine
G Guanine
$\mathrm{T}$ Thymine
U Uracil

References to and abbreviations of amino acids follow the recommendations of IUPAC (International Union of Pure and Applied Chemistry) and IUB (International Union of Biochemistry and Molecular Biology):

$\begin{array}{llllll}\text { A } & \text { Ala } & \text { Alanine } & \text { M } & \text { Met } & \text { Methionine } \\ \text { C } & \text { Cys } & \text { Cysteine } & \text { N } & \text { Asn } & \text { Asparagine } \\ \text { D } & \text { Asp } & \text { Aspartic acid } & \text { P } & \text { Pro } & \text { Proline } \\ \text { E } & \text { Glu } & \text { Glutamic acid } & \text { Q } & \text { Gln } & \text { Glutamine } \\ \text { F } & \text { Phe } & \text { Phenylalanine } & \text { R } & \text { Arg } & \text { Arginine } \\ \text { G } & \text { Gly } & \text { Glycine } & \text { S } & \text { Ser } & \text { Serine } \\ \text { H } & \text { His } & \text { Histidine } & \text { T } & \text { Thr } & \text { Threonine } \\ \text { I } & \text { Ile } & \text { Isoleucine } & \text { V } & \text { Val } & \text { Valine } \\ \text { K } & \text { Lys } & \text { Lysine } & \text { W } & \text { Trp } & \text { Tryptophan } \\ \text { L } & \text { Leu } & \text { Leucine } & \text { Y } & \text { Tyr } & \text { Tyrosine } \\ & & & \text { X } & \text { Xaa } & \text { Unspecified amino acid }\end{array}$




\section{Bibliography}

[1] B. Alberts, A. Johnson, J. Lewis, and M. Raff. Molecular Biology of the Cell. Garland Science, 4 edition, 2002.

[2] G. J. Siegel, editor. Basic Neurochemistry: Molecular, Cellular and Medical Aspects. Lippincott Williams \& Wilkins, 6th edition, 1998.

[3] D. K. Hartline and D. R. Colman. Rapid conduction and the evolution of giant axons and myelinated fibers. Curr Biol, 17(1):R29-R35, Jan 2007.

[4] C. Hildebrand, S. Remahl, H. Persson, and C. Bjartmar. Myelinated nerve fibres in the cns. Prog Neurobiol, 40(3):319-384, Mar 1993.

[5] K.-A. Nave. Myelination and the trophic support of long axons. Nat Rev Neurosci, 11(4):275-283, Apr 2010.

[6] B. Zalc and D. R. Colman. Origins of vertebrate success. Science, 288(5464):271272, Apr 2000.

[7] B. I. Roots. The phylogeny of invertebrates and the evolution of myelin. Neuron Glia Biol, 4(2):101-109, May 2008.

[8] J. E. Heuser and C. F. Doggenweiler. The fine structural organization of nerve fibers, sheaths, and glial cells in the prawn, palaemonetes vulgaris. J Cell Biol, 30(2):381-403, Aug 1966.

[9] B. Geren. The formation from the schwann cell surface of myelin in the peripheral nerves of chick embryos. Exp. Cell Res., 7:558-562, 1954.

[10] A. Peters. The formation and structure of myelin sheaths in the central nervous system. J Biophys Biochem Cytol, 8:431-446, Oct 1960.

[11] S. Remahl and C. Hildebrand. Changing relation between onset of myelination and axon diameter range in developing feline white matter. J Neurol Sci, 54(1):33-45, Apr 1982. 
[12] N. Baumann and D. Pham-Dinh. Biology of oligodendrocyte and myelin in the mammalian central nervous system. Physiol Rev, 81(2):871-927, Apr 2001.

[13] J. L. Salzer. Polarized domains of myelinated axons. Neuron, 40(2):297-318, Oct 2003.

[14] C. Hildebrand and S. G. Waxman. Postnatal differentiation of rat optic nerve fibers: electron microscopic observations on the development of nodes of ranvier and axoglial relations. J Comp Neurol, 224(1):25-37, Mar 1984.

[15] J. Rosenbluth. Multiple functions of the paranodal junction of myelinated nerve fibers. J Neurosci Res, 87(15):3250-3258, Nov 2009.

[16] M. A. Wells and J. C. Dittmer. A comprehensive study of the postnatal changes in the concentration of the lipids of developing rat brain. Biochemistry, 6(10):31693175 , Oct 1967.

[17] H. C. Kinney, B. A. Brody, A. S. Kloman, and F. H. Gilles. Sequence of central nervous system myelination in human infancy. ii. patterns of myelination in autopsied infants. J Neuropathol Exp Neurol, 47(3):217-234, May 1988.

[18] S. Remahl and C. Hildebrand. Relations between axons and oligodendroglial cells during initial myelination. ii. the individual axon. J Neurocytol, 19(6):883-898, Dec 1990.

[19] S. Remahl and C. Hilderbrand. Relation between axons and oligodendroglial cells during initial myelination. i. the glial unit. J Neurocytol, 19(3):313-328, Jun 1990.

[20] C. Hildebrand, S. Remahl, and S. G. Waxman. Axo-glial relations in the retina-optic nerve junction of the adult rat: electron-microscopic observations. J Neurocytol, 14(4):597-617, Aug 1985.

[21] S. G. Waxman and R. J. Melker. Closely spaced nodes of ranvier in the mammalian brain. Brain Res, 32(2):445-448, Sep 1971.

[22] C. H. Berthold and T. Carlstedt. Observations on the morphology at the transition between the peripheral and the central nervous system in the cat. ii. general organization of the transitional region in s1 dorsal rootlets. Acta Physiol Scand Suppl, 446:23-42, 1977.

[23] S. Remahl and C. Hildebrand. Myelinated non-axonal neuronal elements in the feline olfactory bulb lack sites with a nodal structural differentiation. Brain Res, 325(1-2):1-11, Jan 1985. 
[24] B. Barres. The mystery and magic of glia: a perspective on their roles in health and disease. Neuron, 60(3):430-440, Nov 2008.

[25] R. Virchow. Gesammelte abhandlungen zur wissenschaftichen medicin. Hamm, Frankfurt a.M., page p. 507, 1856.

[26] G. G. Somjen. Nervenkitt: notes on the history of the concept of neuroglia. Glia, $1(1): 2-9,1988$.

[27] A. S. Gill and D. K. Binder. Wilder penfield, pío del río-hortega, and the discovery of oligodendroglia. Neurosurgery, 60(5):940-8; discussion 940-8, May 2007.

[28] A. Vallstedt, J. M. Klos, and J. Ericson. Multiple dorsoventral origins of oligodendrocyte generation in the spinal cord and hindbrain. Neuron, 45(1):55-67, Jan 2005 .

[29] W. D. Richardson, N. Kessaris, and N. Pringle. Oligodendrocyte wars. Nat Rev Neurosci, 7(1):11-18, Jan 2006.

[30] J. Cai, Y. Qi, X. Hu, M. Tan, Z. Liu, J. Zhang, Q. Li, M. Sander, and M. Qiu. Generation of oligodendrocyte precursor cells from mouse dorsal spinal cord independent of nkx6 regulation and shh signaling. Neuron, 45(1):41-53, Jan 2005.

[31] M. Fogarty, W. D. Richardson, and N. Kessaris. A subset of oligodendrocytes generated from radial glia in the dorsal spinal cord. Development, 132(8):1951-1959, Apr 2005.

[32] W. D. Richardson, H. K. Smith, T. Sun, N. P. Pringle, A. Hall, and R. Woodruff. Oligodendrocyte lineage and the motor neuron connection. Glia, 29(2):136-142, Jan 2000 .

[33] N. Kessaris, M. Fogarty, P. Iannarelli, M. Grist, M. Wegner, and W. D. Richardson. Competing waves of oligodendrocytes in the forebrain and postnatal elimination of an embryonic lineage. Nat Neurosci, 9(2):173-179, Feb 2006.

[34] D. J. Nicolay, J. R. Doucette, and A. J. Nazarali. Transcriptional control of oligodendrogenesis. Glia, 55(13):1287-1299, Oct 2007.

[35] M. Noble. Precursor cell transitions in oligodendrocyte development. J Cell Biol, 148(5):839-842, Mar 2000.

[36] M. C. Raff, R. H. Miller, and M. Noble. A glial progenitor cell that develops in vitro into an astrocyte or an oligodendrocyte depending on culture medium. Nature, 303(5916):390-396, 1983. 
[37] S. E. Pfeiffer, A. E. Warrington, and R. Bansal. The oligodendrocyte and its many cellular processes. Trends Cell Biol, 3(6):191-197, Jun 1993.

[38] A. M. Butt, M. Ibrahim, and M. Berry. Axon-myelin sheath relations of oligodendrocyte unit phenotypes in the adult rat anterior medullary velum. $J$ Neurocytol, 27(4):259-269, Apr 1998.

[39] I. Sommer and M. Schachner. Monoclonal antibodies (o1 to o4) to oligodendrocyte cell surfaces: an immunocytological study in the central nervous system. Dev Biol, 83(2):311-327, Apr 1981.

[40] C. M. Kassmann, C. Lappe-Siefke, M. Baes, B. Brügger, A. Mildner, H. B. Werner, O. Natt, T. Michaelis, M. Prinz, J. Frahm, and K.-A. Nave. Axonal loss and neuroinflammation caused by peroxisome-deficient oligodendrocytes. Nat Genet, 39(8):969976, Aug 2007.

[41] A. Teigler, D. Komljenovic, A. Draguhn, K. Gorgas, and W. W. Just. Defects in myelination, paranode organization and purkinje cell innervation in the ether lipiddeficient mouse cerebellum. Hum Mol Genet, 18(11):1897-1908, Jun 2009.

[42] G. J. Snipes and U. Suter. Cholesterol and myelin. Subcell Biochem, 28:173-204, 1997.

[43] W. T. Norton and W. Cammer. Myelin, chapter Isolation and characterization of myelin, pages 147-195. Plenum Press, New York., 1984.

[44] J. S. O'Brien. Stability of the myelin membrane. Science, 147:1099-1107, Mar 1965.

[45] J. S. O'Brien and E. L. Sampson. Lipid composition of the normal human brain: gray matter, white matter, and myelin. J Lipid Res, 6(4):537-544, Oct 1965.

[46] W. T. Norton and L. A. Autilio. The lipid composition of purified bovine brain myelin. J Neurochem, 13(4):213-222, Apr 1966.

[47] G. van Meer, D. R. Voelker, and G. W. Feigenson. Membrane lipids: where they are and how they behave. Nat Rev Mol Cell Biol, 9(2):112-124, Feb 2008.

[48] S. Degroote, J. Wolthoorn, and G. van Meer. The cell biology of glycosphingolipids. Semin Cell Dev Biol, 15(4):375-387, Aug 2004.

[49] G. van Meer and Q. Lisman. Sphingolipid transport: rafts and translocators. J Biol Chem, 277(29):25855-25858, Jul 2002. 
[50] S. Schulte and W. Stoffel. Ceramide udpgalactosyltransferase from myelinating rat brain: purification, cloning, and expression. Proc Natl Acad Sci U S A, 90(21):1026510269, Nov 1993.

[51] H. Sprong, B. Kruithof, R. Leijendekker, J. W. Slot, G. van Meer, and P. van der Sluijs. Udp-galactose:ceramide galactosyltransferase is a class i integral membrane protein of the endoplasmic reticulum. J Biol Chem, 273(40):25880-25888, Oct 1998.

[52] X. Q. Wang, P. Sun, and A. S. Paller. Inhibition of integrin-linked kinase/protein kinase b/akt signaling: mechanism for ganglioside-induced apoptosis. J Biol Chem, 276(48):44504-44511, Nov 2001.

[53] A. A. Farooqui, T. Farooqui, A. J. Yates, and L. A. Horrocks. Regulation of protein kinase c activity by various lipids. Neurochem Res, 13(6):499-511, Jun 1988.

[54] K. Simons and G. van Meer. Lipid sorting in epithelial cells. Biochemistry, 27(17):6197-6202, Aug 1988.

[55] W. I. Lencer, T. R. Hirst, and R. K. Holmes. Membrane traffic and the cellular uptake of cholera toxin. Biochim Biophys Acta, 1450(3):177-190, Jul 1999.

[56] J. M. Boggs, W. Gao, and Y. Hirahara. Myelin glycosphingolipids, galactosylceramide and sulfatide, participate in carbohydrate-carbohydrate interactions between apposed membranes and may form glycosynapses between oligodendrocyte and/or myelin membranes. Biochim Biophys Acta, 1780(3):445-455, Mar 2008.

[57] A. Bosio, E. Binczek, and W. Stoffel. Functional breakdown of the lipid bilayer of the myelin membrane in central and peripheral nervous system by disrupted galactocerebroside synthesis. Proc Natl Acad Sci U S A, 93(23):13280-13285, Nov 1996.

[58] T. Coetzee, N. Fujita, J. Dupree, R. Shi, A. Blight, K. Suzuki, K. Suzuki, and B. Popko. Myelination in the absence of galactocerebroside and sulfatide: normal structure with abnormal function and regional instability. Cell, 86(2):209-219, Jul 1996.

[59] J. L. Dupree, T. Coetzee, A. Blight, K. Suzuki, and B. Popko. Myelin galactolipids are essential for proper node of ranvier formation in the cns. J Neurosci, 18(5):16421649, Mar 1998.

[60] B. Popko, J. L. Dupree, T. Coetzee, K. Suzuki, and K. Suzuki. Genetic analysis of myelin galactolipid function. Adv Exp Med Biol, 468:237-244, 1999. 
[61] S. Imgrund, D. Hartmann, H. Farwanah, M. Eckhardt, R. Sandhoff, J. Degen, V. Gieselmann, K. Sandhoff, and K. Willecke. Adult ceramide synthase 2 (cers2)deficient mice exhibit myelin sheath defects, cerebellar degeneration, and hepatocarcinomas. J Biol Chem, 284(48):33549-33560, Nov 2009.

[62] K. Suzuki. Globoid cell leukodystrophy (krabbe's disease): update. J Child Neurol, 18(9):595-603, Sep 2003.

[63] V. Gieselmann. Metachromatic leukodystrophy: recent research developments. $J$ Child Neurol, 18(9):591-594, Sep 2003.

[64] Peter J Espenshade and Adam L Hughes. Regulation of sterol synthesis in eukaryotes. Annu Rev Genet, 41:401-427, 2007.

[65] A. N. Davison. Brain sterol metabolism. Adv Lipid Res, 3:171-196, 1965.

[66] R. H. Laatsch, M. W. Kies, S. Gordon, and E. C. Alvord. The encephalomyelitic activity of myelin isolated by ultracentrifugation. J Exp Med, 115:777-788, Apr 1962.

[67] J. M. Dietschy and S. D. Turley. Thematic review series: brain lipids. cholesterol metabolism in the central nervous system during early development and in the mature animal. J Lipid Res, 45(8):1375-1397, Aug 2004.

[68] D. K. Spady and J. M. Dietschy. Sterol synthesis in vivo in 18 tissues of the squirrel monkey, guinea pig, rabbit, hamster, and rat. J Lipid Res, 24(3):303-315, Mar 1983.

[69] G. Saher, B. Brügger, C. Lappe-Siefke, W. Möbius, R. Tozawa, M. C. Wehr, F. Wieland, S. Ishibashi, and K.-A. Nave. High cholesterol level is essential for myelin membrane growth. Nat Neurosci, 8(4):468-475, Apr 2005.

[70] K. Simons and E. Ikonen. Functional rafts in cell membranes. Nature, 387(6633):569572, Jun 1997.

[71] G. Bu. Apolipoprotein e and its receptors in alzheimer's disease: pathways, pathogenesis and therapy. Nat Rev Neurosci, 10(5):333-344, May 2009.

[72] O. Jahn, S. Tenzer, and H. B. Werner. Myelin proteomics: molecular anatomy of an insulating sheath. Mol Neurobiol, 40(1):55-72, Aug 2009.

[73] D. A. Kirschner, A. L. Ganser, and D. L. D. Caspar. Myelin, chapter Diffraction studies of molecular organization and membrane interactions in myelin, pages 51-95. Plenum Press, New York, 1984. 
[74] J. M. Boggs. Myelin basic protein: a multifunctional protein. Cell Mol Life Sci, 63(17):1945-1961, Sep 2006.

[75] G. F. Chernoff. Shiverer: an autosomal recessive mutant mouse with myelin deficiency. J Hered, 72(2):128, 1981.

[76] A. Privat, C. Jacque, J. M. Bourre, P. Dupouey, and N. Baumann. Absence of the major dense line in myelin of the mutant mouse "shiverer". Neurosci Lett, 12(1):107-112, Apr 1979.

[77] K. Mikoshiba, S. Kohsaka, K. Takamatsu, and Y. Tsukada. Neurochemical and morphological studies on the myelin of peripheral nervous system from shiverer mutant mice: absence of basic proteins common to central nervous system. Brain Res, 204(2):455-460, Jan 1981.

[78] A. M. Messier and O. A. Bizzozero. Conserved fatty acid composition of proteolipid protein during brain development and in myelin subfractions. Neurochem Res, 25(4):449-455, Apr 2000.

[79] J. L. Popot, D. Pham Dinh, and A. Dautigny. Major myelin proteolipid: the 4alpha-helix topology. J Membr Biol, 123(3):278, Sep 1991.

[80] Judith M Greer and Marjorie B Lees. Myelin proteolipid protein-the first 50 years. Int J Biochem Cell Biol, 34(3):211-215, Mar 2002.

[81] K. A. Nave. Neurological mouse mutants and the genes of myelin. J Neurosci Res, 38(6):607-612, Aug 1994.

[82] M. Klugmann, M. H. Schwab, A. Pühlhofer, A. Schneider, F. Zimmermann, I. R. Griffiths, and K. A. Nave. Assembly of cns myelin in the absence of proteolipid protein. Neuron, 18(1):59-70, Jan 1997.

[83] H. Jurevics, J. Hostettler, D. W. Sammond, K.-A. Nave, A. D. Toews, and P. Morell. Normal metabolism but different physical properties of myelin from mice deficient in proteolipid protein. J Neurosci Res, 71(6):826-834, Mar 2003.

[84] T. J. Anderson, A. Schneider, J. A. Barrie, M. Klugmann, M. C. McCulloch, D. Kirkham, E. Kyriakides, K. A. Nave, and I. R. Griffiths. Late-onset neurodegeneration in mice with increased dosage of the proteolipid protein gene. J Comp Neurol, 394(4):506-519, May 1998.

[85] I. Griffiths, M. Klugmann, T. Anderson, C. Thomson, D. Vouyiouklis, and K. A. Nave. Current concepts of plp and its role in the nervous system. Microsc Res Tech, 41(5):344-358, Jun 1998. 
[86] R. H. Quarles. Myelin-associated glycoprotein in development and disease. Dev Neurosci, 6(6):285-303, 1983.

[87] J. L. Salzer, W. P. Holmes, and D. R. Colman. The amino acid sequences of the myelin-associated glycoproteins: homology to the immunoglobulin gene superfamily. J Cell Biol, 104(4):957-965, Apr 1987.

[88] H. Ishiguro, S. Sato, N. Fujita, T. Inuzuka, R. Nakano, and T. Miyatake. Immunohistochemical localization of myelin-associated glycoprotein isoforms during the development in the mouse brain. Brain Res, 563(1-2):288-292, Nov 1991.

[89] T. Inuzuka, N. Fujita, S. Sato, H. Baba, R. Nakano, H. Ishiguro, and T. Miyatake. Expression of the large myelin-associated glycoprotein isoform during the development in the mouse peripheral nervous system. Brain Res, 562(1):173-175, Oct 1991.

[90] N. H. Sternberger, R. H. Quarles, Y. Itoyama, and H. D. Webster. Myelin-associated glycoprotein demonstrated immunocytochemically in myelin and myelin-forming cells of developing rat. Proc Natl Acad Sci U S A, 76(3):1510-1514, Mar 1979.

[91] R. L. Schnaar, B. E. Collins, L. P. Wright, M. Kiso, M. B. Tropak, J. C. Roder, and P. R. Crocker. Myelin-associated glycoprotein binding to gangliosides. structural specificity and functional implications. Ann N Y Acad Sci, 845:92-105, Jun 1998.

[92] H. Umemori, S. Sato, T. Yagi, S. Aizawa, and T. Yamamoto. Initial events of myelination involve fyn tyrosine kinase signalling. Nature, 367(6463):572-576, Feb 1994.

[93] M. Domeniconi, Z. Cao, T. Spencer, R. Sivasankaran, K. Wang, E. Nikulina, N. Kimura, H. Cai, K. Deng, Y. Gao, Z. He, and M. Filbin. Myelin-associated glycoprotein interacts with the nogo66 receptor to inhibit neurite outgrowth. Neuron, 35(2):283-290, Jul 2002.

[94] F. Orfaniotou. A novel role for myelin-associated inhibitors in modulating microglial motility. PhD thesis, Georg August University Goettingen, 2008.

[95] D. Montag, K. P. Giese, U. Bartsch, R. Martini, Y. Lang, H. Blüthmann, J. Karthigasan, D. A. Kirschner, E. S. Wintergerst, and K. A. Nave. Mice deficient for the myelin-associated glycoprotein show subtle abnormalities in myelin. Neuron, 13(1):229-246, Jul 1994.

[96] C. Li, M. B. Tropak, R. Gerlai, S. Clapoff, W. Abramow-Newerly, B. Trapp, A. Peterson, and J. Roder. Myelination in the absence of myelin-associated glycoprotein. Nature, 369(6483):747-750, Jun 1994. 
[97] M. C. Birling, G. Roussel, F. Nussbaum, and J. L. Nussbaum. Biochemical and immunohistochemical studies with specific polyclonal antibodies directed against bovine myelin/oligodendrocyte glycoprotein. Neurochem Res, 18(8):937-945, Aug 1993.

[98] D. Pham-Dinh, M. G. Mattei, J. L. Nussbaum, G. Roussel, P. Pontarotti, N. Roeckel, I. H. Mather, K. Artzt, K. F. Lindahl, and A. Dautigny. Myelin/oligodendrocyte glycoprotein is a member of a subset of the immunoglobulin superfamily encoded within the major histocompatibility complex. Proc Natl Acad Sci U S A, 90(17):7990-7994, Sep 1993.

[99] J. F. Kroepfl, L. R. Viise, A. J. Charron, C. Linington, and M. V. Gardinier. Investigation of myelin/oligodendrocyte glycoprotein membrane topology. J Neurochem, 67(5):2219-2222, Nov 1996.

[100] C. Brunner, H. Lassmann, T. V. Waehneldt, J. M. Matthieu, and C. Linington. Differential ultrastructural localization of myelin basic protein, myelin/oligodendroglial glycoprotein, and 2',3'-cyclic nucleotide 3'-phosphodiesterase in the cns of adult rats. J Neurochem, 52(1):296-304, Jan 1989.

[101] T. G. Johns and C. C. Bernard. The structure and function of myelin oligodendrocyte glycoprotein. J Neurochem, 72(1):1-9, Jan 1999.

[102] C. Delarasse, P. Daubas, L. T. Mars, C. Vizler, T. Litzenburger, A. Iglesias, J. Bauer, B. D. Gaspera, A. Schubart, L. Decker, D. Dimitri, G. Roussel, A. Dierich, S. Amor, A. Dautigny, R. Liblau, and D. Pham-Dinh. Myelin/oligodendrocyte glycoproteindeficient (mog-deficient) mice reveal lack of immune tolerance to mog in wild-type mice. J Clin Invest, 112(4):544-553, Aug 2003.

[103] T. Kurihara, K. Monoh, K. Sakimura, and Y. Takahashi. Alternative splicing of mouse brain 2',3'-cyclic-nucleotide 3'-phosphodiesterase mrna. Biochem Biophys Res Commun, 170(3):1074-1081, Aug 1990.

[104] R. C. O'Neill, J. Minuk, M. E. Cox, P. E. Braun, and M. Gravel. Cnp2 mrna directs synthesis of both cnp1 and cnp2 polypeptides. J Neurosci Res, 50(2):248-257, Oct 1997.

[105] P. E. Braun, D. De Angelis, W. W. Shtybel, and L. Bernier. Isoprenoid modification permits 2',3'-cyclic nucleotide 3'-phosphodiesterase to bind to membranes. $J$ Neurosci Res, 30(3):540-544, Nov 1991. 
[106] B. D. Trapp, L. Bernier, S. B. Andrews, and D. R. Colman. Cellular and subcellular distribution of 2',3'-cyclic nucleotide 3'-phosphodiesterase and its mrna in the rat central nervous system. J Neurochem, 51(3):859-868, Sep 1988.

[107] J. Lee, M. Gravel, R. Zhang, P. Thibault, and P. E. Braun. Process outgrowth in oligodendrocytes is mediated by cnp, a novel microtubule assembly myelin protein. J Cell Biol, 170(4):661-673, Aug 2005.

[108] C. Lappe-Siefke, S. Goebbels, M. Gravel, E. Nicksch, J. Lee, P. E. Braun, I. R. Griffiths, and K.-A. Nave. Disruption of cnp1 uncouples oligodendroglial functions in axonal support and myelination. Nat Genet, 33(3):366-374, Mar 2003.

[109] M. N. Rasband, J. T., Y. Kaga, Y. Yang, C. Lappe-Siefke, K.-A. Nave, and R. Bansal. Cnp is required for maintenance of axon-glia interactions at nodes of ranvier in the cns. Glia, 50(1):86-90, Apr 2005.

[110] M. Gravel, J. Peterson, V. W. Yong, V. Kottis, B. Trapp, and P. E. Braun. Overexpression of 2',3'-cyclic nucleotide 3'-phosphodiesterase in transgenic mice alters oligodendrocyte development and produces aberrant myelination. Mol Cell Neurosci, 7(6):453-466, Jun 1996.

[111] J. M. Bronstein. Function of tetraspan proteins in the myelin sheath. Curr Opin Neurobiol, 10(5):552-557, Oct 2000.

[112] J. M. Bronstein, P. E. Micevych, and K. Chen. Oligodendrocyte-specific protein (osp) is a major component of cns myelin. J Neurosci Res, 50(5):713-720, Dec 1997.

[113] E. Chow, J. Mottahedeh, M. Prins, W. Ridder, S. Nusinowitz, and J. M. Bronstein. Disrupted compaction of cns myelin in an osp/claudin-11 and plp/dm20 double knockout mouse. Mol Cell Neurosci, 29(3):405-413, Jul 2005.

[114] S. S. Scherer, S. M. Deschênes, Y. T. Xu, J. B. Grinspan, K. H. Fischbeck, and D. L. Paul. Connexin32 is a myelin-related protein in the pns and cns. J Neurosci, 15(12):8281-8294, Dec 1995.

[115] M. Erb, A. J. Steck, K. A. Nave, and N. Schaeren-Wiemers. Differential expression of l- and s-mag upon camp stimulated differentiation in oligodendroglial cells. $J$ Neurosci Res, 71(3):326-337, Feb 2003.

[116] M. Frank. Mal, a proteolipid in glycosphingolipid enriched domains: functional implications in myelin and beyond. Prog Neurobiol, 60(6):531-544, Apr 2000. 
[117] K. H. Cheong, D. Zacchetti, E. E. Schneeberger, and K. Simons. Vip17/mal, a lipid raft-associated protein, is involved in apical transport in mdck cells. Proc Natl Acad Sci U S A, 96(11):6241-6248, May 1999.

[118] A. Holz and M. E. Schwab. Developmental expression of the myelin gene mobp in the rat nervous system. J Neurocytol, 26(7):467-477, Jul 1997.

[119] P. Montague, A. S. McCallion, R. W. Davies, and I. R. Griffiths. Myelin-associated oligodendrocytic basic protein: a family of abundant cns myelin proteins in search of a function. Dev Neurosci, 28(6):479-487, 2006.

[120] P. Montague, P. J. Dickinson, A. S. McCallion, G. J. Stewart, A. Savioz, R. W. Davies, P. G. Kennedy, and I. R. Griffiths. Developmental expression of the murine mobp gene. J Neurosci Res, 49(2):133-143, Jul 1997.

[121] G. Palade. Intracellular aspects of the process of protein synthesis. Science, 189(4200):347-358, Aug 1975.

[122] J. S. Bonifacino and B. S. Glick. The mechanisms of vesicle budding and fusion. Cell, 116(2):153-166, Jan 2004.

[123] N. B. Cole and J. Lippincott-Schwartz. Organization of organelles and membrane traffic by microtubules. Curr Opin Cell Biol, 7(1):55-64, Feb 1995.

[124] R. B. Kelly. Microtubules, membrane traffic, and cell organization. Cell, 61(1):5-7, Apr 1990.

[125] L. Ellgaard and A. Helenius. Er quality control: towards an understanding at the molecular level. Curr Opin Cell Biol, 13(4):431-437, Aug 2001.

[126] B. S. Glick. Organization of the golgi apparatus. Curr Opin Cell Biol, 12(4):450-456, Aug 2000.

[127] T. E. Kreis, M. Lowe, and R. Pepperkok. Cops regulating membrane traffic. Annu Rev Cell Dev Biol, 11:677-706, 1995.

[128] K. Honke, M. Tsuda, Y. Hirahara, A. Ishii, A. Makita, and Y. Wada. Molecular cloning and expression of cdna encoding human 3'phosphoadenylylsulfate:galactosylceramide 3'-sulfotransferase. $J$ Biol Chem, 272(8):4864-4868, Feb 1997.

[129] G. Griffiths and K. Simons. The trans golgi network: sorting at the exit site of the golgi complex. Science, 234(4775):438-443, Oct 1986. 
[130] M. Simons, E. M. Krämer, C. Thiele, W. Stoffel, and J. Trotter. Assembly of myelin by association of proteolipid protein with cholesterol- and galactosylceramide-rich membrane domains. J Cell Biol, 151(1):143-154, Oct 2000.

[131] R. W. Klemm, C. S. Ejsing, M. A. Surma, H.-J. Kaiser, M. J. Gerl, J. L. Sampaio, Q. de Robillard, C. Ferguson, T. J. Proszynski, A. Shevchenko, and K. Simons. Segregation of sphingolipids and sterols during formation of secretory vesicles at the trans-golgi network. J Cell Biol, 185(4):601-612, May 2009.

[132] A. L. Ang, T. Taguchi, S. Francis, H. Fölsch, L. J. Murrells, M. Pypaert, G. Warren, and I. Mellman. Recycling endosomes can serve as intermediates during transport from the golgi to the plasma membrane of mdck cells. J Cell Biol, 167(3):531-543, Nov 2004.

[133] A. Musch. Microtubule organization and function in epithelial cells. Traffic, 5(1):19, Jan 2004.

[134] P. Keller, D. Toomre, E. Díaz, J. White, and K. Simons. Multicolour imaging of post-golgi sorting and trafficking in live cells. Nat Cell Biol, 3(2):140-149, Feb 2001.

[135] S. Schuck and K. Simons. Polarized sorting in epithelial cells: raft clustering and the biogenesis of the apical membrane. J Cell Sci, 117(Pt 25):5955-5964, Dec 2004.

[136] G. S. Bloom and L. S. Goldstein. Cruising along microtubule highways: how membranes move through the secretory pathway. J Cell Biol, 140(6):1277-1280, Mar 1998.

[137] J. L. Ross, M. Y. Ali, and D. M. Warshaw. Cargo transport: molecular motors navigate a complex cytoskeleton. Curr Opin Cell Biol, 20(1):41-47, Feb 2008.

[138] R. Jahn and T. C. Südhof. Membrane fusion and exocytosis. Annu Rev Biochem, 68:863-911, 1999.

[139] R. Jahn and R. H. Scheller. Snares-engines for membrane fusion. Nat Rev Mol Cell Biol, 7(9):631-643, Sep 2006.

[140] H. Stenmark and V. M. Olkkonen. The rab gtpase family. Genome Biol, 2(5):REVIEWS3007, 2001.

[141] J. H. Lipschutz and K. E. Mostov. Exocytosis: the many masters of the exocyst. Curr Biol, 12(6):R212-R214, Mar 2002.

[142] M. A. De Matteis and A. Godi. Pi-loting membrane traffic. Nat Cell Biol, 6(6):487492, Jun 2004. 
[143] D. R. TerBush, T. Maurice, D. Roth, and P. Novick. The exocyst is a multiprotein complex required for exocytosis in saccharomyces cerevisiae. EMBO J, 15(23):64836494, Dec 1996.

[144] K. K. Grindstaff, C. Yeaman, N. Anandasabapathy, S. C. Hsu, E. Rodriguez-Boulan, R. H. Scheller, and W. J. Nelson. Sec6/8 complex is recruited to cell-cell contacts and specifies transport vesicle delivery to the basal-lateral membrane in epithelial cells. Cell, 93(5):731-740, May 1998.

[145] C. D. Hazuka, D. L. Foletti, S. C. Hsu, Y. Kee, F. W. Hopf, and R. H. Scheller. The sec6/8 complex is located at neurite outgrowth and axonal synapse-assembly domains. J Neurosci, 19(4):1324-1334, Feb 1999.

[146] H. Stenmark. Rab gtpases as coordinators of vesicle traffic. Nat Rev Mol Cell Biol, 10(8):513-525, Aug 2009.

[147] S. R. Pfeffer. Rab gtpases: specifying and deciphering organelle identity and function. Trends Cell Biol, 11(12):487-491, Dec 2001.

[148] J. S. Anant, L. Desnoyers, M. Machius, B. Demeler, J. C. Hansen, K. D. Westover, J. Deisenhofer, and M. C. Seabra. Mechanism of rab geranylgeranylation: formation of the catalytic ternary complex. Biochemistry, 37(36):12559-12568, Sep 1998.

[149] J. B. Bock, H. T. Matern, A. A. Peden, and R. H. Scheller. A genomic perspective on membrane compartment organization. Nature, 409(6822):839-841, Feb 2001.

[150] M. Zerial and H. McBride. Rab proteins as membrane organizers. Nat Rev Mol Cell Biol, 2(2):107-117, Feb 2001.

[151] J. C. Hay and R. H. Scheller. Snares and nsf in targeted membrane fusion. Curr Opin Cell Biol, 9(4):505-512, Aug 1997.

[152] T. Weimbs, S. H. Low, S. J. Chapin, K. E. Mostov, P. Bucher, and K. Hofmann. A conserved domain is present in different families of vesicular fusion proteins: a new superfamily. Proc Natl Acad Sci U S A, 94(7):3046-3051, Apr 1997.

[153] W. Hong. Snares and traffic. Biochim Biophys Acta, 1744(2):120-144, Jun 2005.

[154] I. Bethani, T. Lang, U. Geumann, J. J. Sieber, R. Jahn, and S. O. Rizzoli. The specificity of snare pairing in biological membranes is mediated by both proof-reading and spatial segregation. EMBO J, 26(17):3981-3992, Sep 2007. 
[155] D. Fasshauer, R. B. Sutton, A. T. Brunger, and R. Jahn. Conserved structural features of the synaptic fusion complex: Snare proteins reclassified as q- and rsnares. Proc Natl Acad Sci U S A, 95(26):15781-15786, Dec 1998.

[156] E. M. Krämer, A. Schardt, and K. A. Nave. Membrane traffic in myelinating oligodendrocytes. Microsc Res Tech, 52(6):656-671, Mar 2001.

[157] J. J. Sieber, K. I. Willig, R. Heintzmann, S. W. Hell, and T. Lang. The snare motif is essential for the formation of syntaxin clusters in the plasma membrane. Biophys J, 90(8):2843-2851, Apr 2006.

[158] T. F. Martin. $\operatorname{Pi}(4,5) \mathrm{p}(2)$ regulation of surface membrane traffic. Curr Opin Cell Biol, 13(4):493-499, Aug 2001.

[159] I. Milosevic, J. B. Sørensen, T. Lang, M. Krauss, G. Nagy, V. Haucke, R. Jahn, and E. Neher. Plasmalemmal phosphatidylinositol-4,5-bisphosphate level regulates the releasable vesicle pool size in chromaffin cells. J Neurosci, 25(10):2557-2565, Mar 2005 .

[160] Y. Aikawa and T. F. J. Martin. Arf6 regulates a plasma membrane pool of phosphatidylinositol(4,5)bisphosphate required for regulated exocytosis. J Cell Biol, 162(4):647-659, Aug 2003.

[161] A. Radhakrishnan, A. Stein, R. Jahn, and D. Fasshauer. The ca2+ affinity of synaptotagmin 1 is markedly increased by a specific interaction of its $\mathrm{c} 2 \mathrm{~b}$ domain with phosphatidylinositol 4,5-bisphosphate. J Biol Chem, 284(38):25749-25760, Sep 2009.

[162] S. D. Conner and S. L. Schmid. Regulated portals of entry into the cell. Nature, 422(6927):37-44, Mar 2003.

[163] E. J. Ungewickell and L. Hinrichsen. Endocytosis: clathrin-mediated membrane budding. Curr Opin Cell Biol, 19(4):417-425, Aug 2007.

[164] J. Heuser. Three-dimensional visualization of coated vesicle formation in fibroblasts. J Cell Biol, 84(3):560-583, Mar 1980.

[165] T. Kirchhausen. Clathrin. Annu Rev Biochem, 69:699-727, 2000.

[166] T. Itoh, K. S. Erdmann, A. Roux, B. Habermann, H. Werner, and P. De Camilli. Dynamin and the actin cytoskeleton cooperatively regulate plasma membrane invagination by bar and f-bar proteins. Dev Cell, 9(6):791-804, Dec 2005. 
[167] J. Marx. Caveolae: a once-elusive structure gets some respect. Science, 294(5548):1862-1865, Nov 2001.

[168] R. G. Parton and K. Simons. The multiple faces of caveolae. Nat Rev Mol Cell Biol, 8(3):185-194, Mar 2007.

[169] P. Lajoie, J. G. Goetz, J. W. Dennis, and I. R. Nabi. Lattices, rafts, and scaffolds: domain regulation of receptor signaling at the plasma membrane. J Cell Biol, 185(3):381-385, May 2009.

[170] J. R. Henley, E. W. Krueger, B. J. Oswald, and M. A. McNiven. Dynamin-mediated internalization of caveolae. J Cell Biol, 141(1):85-99, Apr 1998.

[171] P. Caroni. New embo members' review: actin cytoskeleton regulation through modulation of pi(4,5)p(2) rafts. EMBO J, 20(16):4332-4336, Aug 2001.

[172] F. R. Maxfield and T. E. McGraw. Endocytic recycling. Nat Rev Mol Cell Biol, 5(2):121-132, Feb 2004.

[173] M. J. Clague. Molecular aspects of the endocytic pathway. Biochem J, 336 ( Pt 2):271-282, Dec 1998.

[174] I. Mellman and W. J. Nelson. Coordinated protein sorting, targeting and distribution in polarized cells. Nat Rev Mol Cell Biol, 9(11):833-845, Nov 2008.

[175] O. A. Weisz and E. Rodriguez-Boulan. Apical trafficking in epithelial cells: signals, clusters and motors. J Cell Sci, 122(Pt 23):4253-4266, Dec 2009.

[176] A. C. Horton and M. D. Ehlers. Neuronal polarity and trafficking. Neuron, 40(2):277-295, Oct 2003.

[177] K. H. Pfenninger. Plasma membrane expansion: a neuron's herculean task. Nat Rev Neurosci, 10(4):251-261, Apr 2009.

[178] J. F. Kroepfl and M. V. Gardinier. Mutually exclusive apicobasolateral sorting of two oligodendroglial membrane proteins, proteolipid protein and myelin/oligodendrocyte glycoprotein, in madin-darby canine kidney cells. J Neurosci Res, 66(6):1140-1148, Dec 2001.

[179] W. Baron and D. Hoekstra. On the biogenesis of myelin membranes: sorting, trafficking and cell polarity. FEBS Lett, 584(9):1760-1770, May 2010.

[180] M. Anitei, M. Ifrim, M.-A. Ewart, A. E. Cowan, J. H. Carson, R. Bansal, and S. E. Pfeiffer. A role for sec8 in oligodendrocyte morphological differentiation. J Cell Sci, 119(Pt 5):807-818, Mar 2006. 
[181] E. D. Muse, H. Jurevics, A. D. Toews, G. K. Matsushima, and P. Morell. Parameters related to lipid metabolism as markers of myelination in mouse brain. $J$ Neurochem, 76(1):77-86, Jan 2001.

[182] A. Schneider, H. Länder, G. Schulz, H. Wolburg, K.-A. Nave, J. B. Schulz, and M. Simons. Palmitoylation is a sorting determinant for transport to the myelin membrane. J Cell Sci, 118(Pt 11):2415-2423, Jun 2005.

[183] M. C. Brown, M. Besio Moreno, E. R. Bongarzone, P. D. Cohen, E. F. Soto, and J. M. Pasquini. Vesicular transport of myelin proteolipid and cerebroside sulfates to the myelin membrane. J Neurosci Res, 35(4):402-408, Jul 1993.

[184] J. M. Pasquini, M. M. Guarna, M. A. Besio-Moreno, M. T. Iturregui, P. I. Oteiza, and E. F. Soto. Inhibition of the synthesis of glycosphingolipids affects the translocation of proteolipid protein to the myelin membrane. J Neurosci Res, 22(3):289-296, Mar 1989.

[185] R. Bansal and S. E. Pfeiffer. Inhibition of protein and lipid sulfation in oligodendrocytes blocks biological responses to fgf-2 and retards cytoarchitectural maturation, but not developmental lineage progression. Dev Biol, 162(2):511-524, Apr 1994.

[186] H. de Vries and D. Hoekstra. On the biogenesis of the myelin sheath: cognate polarized trafficking pathways in oligodendrocytes. Glycoconj J, 17(3 -4):181-190, 2000 .

[187] J. A. Benjamins, R. Iwata, and J. Hazlett. Kinetics of entry of proteins into the myelin membrane. J Neurochem, 31(4):1077-1085, Oct 1978.

[188] K. Ainger, D. Avossa, F. Morgan, S. J. Hill, C. Barry, E. Barbarese, and J. H. Carson. Transport and localization of exogenous myelin basic protein mrna microinjected into oligodendrocytes. J Cell Biol, 123(2):431-441, Oct 1993.

[189] A. T. Campagnoni, G. D. Carey, and Y. T. Yu. In vitro synthesis of the myelin basic proteins: subcellular site of synthesis. J Neurochem, 34(3):677-686, Mar 1980.

[190] C. Barry, C. Pearson, and E. Barbarese. Morphological organization of oligodendrocyte processes during development in culture and in vivo. Dev Neurosci, 18(4):233$242,1996$.

[191] E. Barbarese, C. Brumwell, S. Kwon, H. Cui, and J. H. Carson. Rna on the road to myelin. J Neurocytol, 28(4-5):263-270, 1999.

[192] J. M. Berg, J. L. Tymoczko, and L. Stryer. Biochemistry. W. H. Freeman, 5 edition, 2002. 
[193] B. Mesmin and F. R. Maxfield. Intracellular sterol dynamics. Biochim Biophys Acta, 1791(7):636-645, Jul 2009.

[194] K. Hanada, K. Kumagai, N. Tomishige, and T. Yamaji. Cert-mediated trafficking of ceramide. Biochim Biophys Acta, 1791(7):684-691, Jul 2009.

[195] T. Hayashi and T.-P. Su. Sigma-1 receptors at galactosylceramide-enriched lipid microdomains regulate oligodendrocyte differentiation. Proc Natl Acad Sci U S A, 101(41):14949-14954, Oct 2004.

[196] M. J. Romanowski, R. E. Soccio, J. L. Breslow, and S. K. Burley. Crystal structure of the mus musculus cholesterol-regulated start protein 4 (stard4) containing a starrelated lipid transfer domain. Proc Natl Acad Sci U S A, 99(10):6949-6954, May 2002 .

[197] B. P. Bouverat, W. H. Krueger, T. Coetzee, R. Bansal, and S. E. Pfeiffer. Expression of rab gtp-binding proteins during oligodendrocyte differentiation in culture. $J$ Neurosci Res, 59(3):446-453, Feb 2000.

[198] A. Schardt, B. G. Brinkmann, M. Mitkovski, M. W. Sereda, H. B. Werner, and K.-A. Nave. The snare protein snap-29 interacts with the gtpase rab3a: Implications for membrane trafficking in myelinating glia. J Neurosci Res, 87(15):3465-3479, Nov 2009 .

[199] A. Feldmann, C. Winterstein, R. White, J. Trotter, and E.-M. Krämer-Albers. Comprehensive analysis of expression, subcellular localization, and cognate pairing of snare proteins in oligodendrocytes. J Neurosci Res, 87(8):1760-1772, Jun 2009.

[200] D. L. Madison, W. H. Krueger, D. Cheng, B. D. Trapp, and S. E. Pfeiffer. Snare complex proteins, including the cognate pair vamp-2 and syntaxin-4, are expressed in cultured oligodendrocytes. J Neurochem, 72(3):988-998, Mar 1999.

[201] K. Trajkovic, A. S. Dhaunchak, J. T. Goncalves, D. Wenzel, A. Schneider, G. Bunt, K.-A. Nave, and M. Simons. Neuron to glia signaling triggers myelin membrane exocytosis from endosomal storage sites. J Cell Biol, 172(6):937-948, Mar 2006.

[202] C. Winterstein, J. Trotter, and E.-M. Krämer-Albers. Distinct endocytic recycling of myelin proteins promotes oligodendroglial membrane remodeling. J Cell Sci, 121(Pt 6):834-842, Mar 2008.

[203] B. D. Trapp, S. B. Andrews, C. Cootauco, and R. Quarles. The myelin-associated glycoprotein is enriched in multivesicular bodies and periaxonal membranes of actively myelinating oligodendrocytes. J Cell Biol, 109(5):2417-2426, Nov 1989. 
[204] R. Wilson and P. J. Brophy. Role for the oligodendrocyte cytoskeleton in myelination. J Neurosci Res, 22(4):439-448, Apr 1989.

[205] J. Song, B. D. Goetz, P. W. Baas, and I. D. Duncan. Cytoskeletal reorganization during the formation of oligodendrocyte processes and branches. Mol Cell Neurosci, 17(4):624-636, Apr 2001.

[206] K. F. Lunn, P. W. Baas, and I. D. Duncan. Microtubule organization and stability in the oligodendrocyte. J Neurosci, 17(13):4921-4932, Jul 1997.

[207] T. Ichimura and M. H. Ellisman. Three-dimensional fine structure of cytoskeletalmembrane interactions at nodes of ranvier. J Neurocytol, 20(8):667-681, Aug 1991.

[208] N. G. Bauer, C. Richter-Landsberg, and C. Ffrench-Constant. Role of the oligodendroglial cytoskeleton in differentiation and myelination. Glia, 57(16):1691-1705, Dec 2009 .

[209] C. Bacon, V. Lakics, L. Machesky, and M. Rumsby. N-wasp regulates extension of filopodia and processes by oligodendrocyte progenitors, oligodendrocytes, and schwann cells-implications for axon ensheathment at myelination. Glia, 55(8):844858, Jun 2007.

[210] X. Liang, N. A. Draghi, and M. D. Resh. Signaling from integrins to fyn to rho family gtpases regulates morphologic differentiation of oligodendrocytes. J Neurosci, 24(32):7140-7149, Aug 2004.

[211] C. Klein, E.-M. Kramer, A.-M. Cardine, B. Schraven, R. Brandt, and J. Trotter. Process outgrowth of oligodendrocytes is promoted by interaction of fyn kinase with the cytoskeletal protein tau. J Neurosci, 22(3):698-707, Feb 2002.

[212] C. M. Southwood, M. Peppi, S. Dryden, M. A. Tainsky, and A. Gow. Microtubule deacetylases, sirt2 and hdac6, in the nervous system. Neurochem Res, 32(2):187-195, Feb 2007.

[213] J. M. Boggs and H. Wang. Co-clustering of galactosylceramide and membrane proteins in oligodendrocyte membranes on interaction with polyvalent carbohydrate and prevention by an intact cytoskeleton. J Neurosci Res, 76(3):342-355, May 2004.

[214] D. Marguet, P.-F. Lenne, H. Rigneault, and H.-T. He. Dynamics in the plasma membrane: how to combine fluidity and order. EMBO J, 25(15):3446-3457, Aug 2006.

[215] S. J. Singer and G. L. Nicolson. The fluid mosaic model of the structure of cell membranes. Science, 175(23):720-731, Feb 1972. 
[216] L. Rajendran and K. Simons. Lipid rafts and membrane dynamics. J Cell Sci, 118(Pt 6):1099-1102, Mar 2005.

[217] P. W. Atkins. Physical Chemistry. W.H.Freeman \& Co Ltd, 1994.

[218] L. A. Bagatolli, J. H. Ipsen, A. C. Simonsen, and O. G. Mouritsen. An outlook on organization of lipids in membranes: searching for a realistic connection with the organization of biological membranes. Prog Lipid Res, May 2010.

[219] E. L. Elson, E. Fried, J. E. Dolbow, and G. M. Genin. Phase separation in biological membranes: integration of theory and experiment. Annu Rev Biophys, 39:207-226, Jun 2010.

[220] R. F. M. de Almeida, A. Fedorov, and M. Prieto. Sphingomyelin/phosphatidylcholine/cholesterol phase diagram: boundaries and composition of lipid rafts. Biophys J, 85(4):2406-2416, Oct 2003.

[221] D. Lingwood and K. Simons. Lipid rafts as a membrane-organizing principle. Science, 327(5961):46-50, Jan 2010.

[222] G. van Meer. Cellular lipidomics. EMBO J, 24(18):3159-3165, Sep 2005.

[223] G. W. Feigenson. Phase boundaries and biological membranes. Annu Rev Biophys Biomol Struct, 36:63-77, 2007.

[224] O. G. Mouritsen and M. Bloom. Mattress model of lipid-protein interactions in membranes. Biophys J, 46(2):141-153, Aug 1984.

[225] M. Sheng and S. H. Lee. Ampa receptor trafficking and the control of synaptic transmission. Cell, 105(7):825-828, Jun 2001.

[226] P.-F. Lenne, L. Wawrezinieck, F. Conchonaud, O. Wurtz, A. Boned, X.-J. Guo, H. Rigneault, H.-T. He, and D. Marguet. Dynamic molecular confinement in the plasma membrane by microdomains and the cytoskeleton meshwork. EMBO J, 25(14):3245-3256, Jul 2006.

[227] K. F. Meiri. Membrane/cytoskeleton communication. Subcell Biochem, 37:247-282, 2004.

[228] C. Eggeling, C. Ringemann, R. Medda, G. Schwarzmann, K. Sandhoff, S. Polyakova, V. N. Belov, B. Hein, C. von Middendorff, A. Schönle, and S. W. Hell. Direct observation of the nanoscale dynamics of membrane lipids in a living cell. Nature, 457(7233):1159-1162, Feb 2009. 
[229] G. R. Chichili and W. Rodgers. Cytoskeleton-membrane interactions in membrane raft structure. Cell Mol Life Sci, 66(14):2319-2328, Jul 2009.

[230] K. Gaus, E. Chklovskaia, B. F. de S. Groth, W. Jessup, and T. Harder. Condensation of the plasma membrane at the site of tymphocyte activation. J Cell Biol, 171(1):121-131, Oct 2005.

[231] A. Gow, C. M. Southwood, J. S. Li, M. Pariali, G. P. Riordan, S. E. Brodie, J. Danias, J. M. Bronstein, B. Kachar, and R. A. Lazzarini. Cns myelin and sertoli cell tight junction strands are absent in osp/claudin-11 null mice. Cell, 99(6):649-659, Dec 1999.

[232] K. Morita, H. Sasaki, K. Fujimoto, M. Furuse, and S. Tsukita. Claudin-11/ospbased tight junctions of myelin sheaths in brain and sertoli cells in testis. $J$ Cell Biol, 145(3):579-588, May 1999.

[233] Ellen Gielen, Wia Baron, Martin Vandeven, Paul Steels, Dick Hoekstra, and Marcel Ameloot. Rafts in oligodendrocytes: evidence and structure-function relationship. Glia, 54(6):499-512, Nov 2006.

[234] R. Puertollano, F. Martín-Belmonte, J. Millán, M. C. de Marco, J. P. Albar, L. Kremer, and M. A. Alonso. The mal proteolipid is necessary for normal apical transport and accurate sorting of the influenza virus hemagglutinin in madin-darby canine kidney cells. J Cell Biol, 145(1):141-151, Apr 1999.

[235] M. Poltorak, R. Sadoul, G. Keilhauer, C. Landa, T. Fahrig, and M. Schachner. Myelin-associated glycoprotein, a member of the 12/hnk-1 family of neural cell adhesion molecules, is involved in neuron-oligodendrocyte and oligodendrocyteoligodendrocyte interaction. J Cell Biol, 105(4):1893-1899, Oct 1987.

[236] S. Kuhlmann-Krieg, I. Sommer, and M. Schachner. Ultrastructural features of cultured oligodendrocytes expressing stage-specific cell-surface antigens. Brain Res, 467(2):269-280, Apr 1988.

[237] J. Trotter and M. Schachner. Cells positive for the o4 surface antigen isolated by cell sorting are able to differentiate into astrocytes or oligodendrocytes. Brain Res Dev Brain Res, 46(1):115-122, Mar 1989.

[238] M. Jung, I. Sommer, M. Schachner, and K. A. Nave. Monoclonal antibody o10 defines a conformationally sensitive cell-surface epitope of proteolipid protein (plp): evidence that plp misfolding underlies dysmyelination in mutant mice. J Neurosci, 16(24):7920-7929, Dec 1996. 
[239] J. M. Greer, C. A. Dyer, M. Pakaski, C. Symonowicz, and M. B. Lees. Orientation of myelin proteolipid protein in the oligodendrocyte cell membrane. Neurochem Res, 21(4):431-440, Apr 1996.

[240] C. Linington and T. V. Waehneldt. Conservation of the carboxyl terminal epitope of myelin proteolipid protein in the tetrapods and lobe-finned fish. J Neurochem, 54(4):1354-1359, Apr 1990.

[241] C. Bourquin, A. Iglesias, T. Berger, H. Wekerle, and C. Linington. Myelin oligodendrocyte glycoprotein-dna vaccination induces antibody-mediated autoaggression in experimental autoimmune encephalomyelitis. Eur J Immunol, 30(12):3663-3671, Dec 2000 .

[242] G. Miesenböck, D. A. De Angelis, and J. E. Rothman. Visualizing secretion and synaptic transmission with ph-sensitive green fluorescent proteins. Nature, 394(6689):192-195, Jul 1998.

[243] P. Liljeström and H. Garoff. A new generation of animal cell expression vectors based on the semliki forest virus replicon. Biotechnology (N Y), 9(12):1356-1361, Dec 1991.

[244] P. Berglund, M. Sjöberg, H. Garoff, G. J. Atkins, B. J. Sheahan, and P. Liljeström. Semliki forest virus expression system: production of conditionally infectious recombinant particles. Biotechnology (N Y), 11(8):916-920, Aug 1993.

[245] D. Toomre, P. Keller, J. White, J. C. Olivo, and K. Simons. Dual-color visualization of trans-golgi network to plasma membrane traffic along microtubules in living cells. J Cell Sci, 112 ( Pt 1):21-33, Jan 1999.

[246] J. Sambrook and D. Russell, editors. Molecular cloning: A laboratory manual. Cold Spring Harbor Laboratory Press, 3 edition, 2001.

[247] A.J. Barrett and G. Salvesen, editors. Proteinase inhibitors. Research monographs in cell and tissue physiology, vol. 12. Elsevier, Amsterdam, 1986.

[248] G. Bertani. Studies on lysogenesis. i. the mode of phage liberation by lysogenic escherichia coli. J Bacteriol, 62(3):293-300, Sep 1951.

[249] H. C. Birnboim and J. Doly. A rapid alkaline extraction procedure for screening recombinant plasmid dna. Nucleic Acids Res, 7(6):1513-1523, Nov 1979.

[250] A. Lehninger, editor. Biochemistry. Worth publishes, Inc, 1975. 
[251] R. B. Helling, H. M. Goodman, and H. W. Boyer. Analysis of endonuclease r-ecori fragments of dna from lambdoid bacteriophages and other viruses by agarose-gel electrophoresis. J Virol, 14(5):1235-1244, Nov 1974.

[252] P. A. Sharp, B. Sugden, and J. Sambrook. Detection of two restriction endonuclease activities in haemophilus parainfluenzae using analytical agarose-ethidium bromide electrophoresis. Biochemistry, 12(16):3055-3063, Jul 1973.

[253] B. Vogelstein and D. Gillespie. Preparative and analytical purification of dna from agarose. Proc Natl Acad Sci U S A, 76(2):615-619, Feb 1979.

[254] W. Arber and D. Dussoix. Host specificity of dna produced by escherichia coli. i. host controlled modification of bacteriophage lambda. J Mol Biol, 5:18-36, Jul 1962.

[255] H. O. Smith and K. W. Wilcox. A restriction enzyme from hemophilus influenzae. i. purification and general properties. J Mol Biol, 51(2):379-391, Jul 1970.

[256] G. H. Sack and D. Nathans. Studies of sv40 dna. vi. cleavage of sv40 dna by restriction endonuclease from hemophilus parainfluenzae. Virology, 51(2):517-520, Feb 1973.

[257] B. Weiss, A. Jacquemin-Sablon, T. R. Live, G. C. Fareed, and C. C. Richardson. Enzymatic breakage and joining of deoxyribonucleic acid. vi. further purification and properties of polynucleotide ligase from escherichia coli infected with bacteriophage t4. J Biol Chem, 243(17):4543-4555, Sep 1968.

[258] K. Mullis, F. Faloona, S. Scharf, R. Saiki, G. Horn, and H. Erlich. Specific enzymatic amplification of dna in vitro: the polymerase chain reaction. Cold Spring Harb Symp Quant Biol, 51 Pt 1:263-273, 1986.

[259] R. K. Saiki, D. H. Gelfand, S. Stoffel, S. J. Scharf, R. Higuchi, G. T. Horn, K. B. Mullis, and H. A. Erlich. Primer-directed enzymatic amplification of dna with a thermostable dna polymerase. Science, 239(4839):487-491, Jan 1988.

[260] K. J. Breslauer, R. Frank, H. Blöcker, and L. A. Marky. Predicting dna duplex stability from the base sequence. Proc Natl Acad Sci U S A, 83(11):3746-3750, Jun 1986.

[261] H. T. Allawi and J. SantaLucia. Thermodynamics and nmr of internal g.t mismatches in dna. Biochemistry, 36(34):10581-10594, Aug 1997.

[262] R. F. Moreira and C. J. Noren. Minimum duplex requirements for restriction enzyme cleavage near the termini of linear dna fragments. Biotechniques, 19(1):56, 58-56, 59, Jul 1995. 
[263] M. H. Caruthers, S. L. Beaucage, C. Becker, J. W. Efcavitch, E. F. Fisher, G. Galluppi, R. Goldman, P. deHaseth, M. Matteucci, and L. McBride. Deoxyoligonucleotide synthesis via the phosphoramidite method. Gene Amplif Anal, 3:1$26,1983$.

[264] C. Papworth, J. C. Bauer, J. Braman, and D. A. Wright. Quikchange site-directed mutagenesis. Strategies, 9:3-4, 1996.

[265] M. Mandel and A. Higa. Calcium-dependent bacteriophage dna infection. $J$ Mol Biol, 53(1):159-162, Oct 1970.

[266] S. N. Cohen, A. C. Chang, and L. Hsu. Nonchromosomal antibiotic resistance in bacteria: genetic transformation of escherichia coli by r-factor dna. Proc Natl Acad Sci U S A, 69(8):2110-2114, Aug 1972.

[267] D. Hanahan. Studies on transformation of escherichia coli with plasmids. J Mol Biol, 166(4):557-580, Jun 1983.

[268] F. Sanger, S. Nicklen, and A. R. Coulson. Dna sequencing with chain-terminating inhibitors. Proc Natl Acad Sci U S A, 74(12):5463-5467, Dec 1977.

[269] P. J. Bredenbeek, I. Frolov, C. M. Rice, and S. Schlesinger. Sindbis virus expression vectors: packaging of rna replicons by using defective helper rnas. J Virol, 67(11):6439-6446, Nov 1993.

[270] M. U. Ehrengruber and K. Lundstrom. Semliki forest virus and sindbis virus vectors. Curr Protoc Hum Genet, Chapter 12:Unit 12.2, Aug 2002.

[271] P. Chomczynski and N. Sacchi. Single-step method of rna isolation by acid guanidinium thiocyanate-phenol-chloroform extraction. Anal Biochem, 162(1):156-159, Apr 1987.

[272] S. Spiegelman, K. F. Watson, and D. L. Kacian. Synthesis of dna complements of natural rnas: a general approach. Proc Natl Acad Sci U S A, 68(11):2843-2845, Nov 1971.

[273] R. Higuchi, C. Fockler, G. Dollinger, and R. Watson. Kinetic pcr analysis: real-time monitoring of dna amplification reactions. Biotechnology (N Y), 11(9):1026-1030, Sep 1993.

[274] H. D. VanGuilder, K. E. Vrana, and W. M. Freeman. Twenty-five years of quantitative pcr for gene expression analysis. Biotechniques, 44(5):619-626, Apr 2008. 
[275] K. J. Livak and T. D. Schmittgen. Analysis of relative gene expression data using real-time quantitative pcr and the 2(-delta delta c(t)) method. Methods, 25(4):402408, Dec 2001.

[276] C. M. Gomez, A. L. Muggleton-Harris, D. G. Whittingham, L. E. Hood, and C. Readhead. Rapid preimplantation detection of mutant (shiverer) and normal alleles of the mouse myelin basic protein gene allowing selective implantation and birth of live young. Proc Natl Acad Sci U S A, 87(12):4481-4484, Jun 1990.

[277] U. K. Laemmli. Cleavage of structural proteins during the assembly of the head of bacteriophage t4. Nature, 227(5259):680-685, Aug 1970.

[278] H. Towbin, T. Staehelin, and J. Gordon. Electrophoretic transfer of proteins from polyacrylamide gels to nitrocellulose sheets: procedure and some applications. Proc Natl Acad Sci U S A, 76(9):4350-4354, Sep 1979.

[279] D. R. Manning, editor. G ProteinsTechniques of Analysis. CRC Press, 1999.

[280] P. Chomczynski. A reagent for the single-step simultaneous isolation of rna, dna and proteins from cell and tissue samples. Biotechniques, 15(3):532-4, 536-7, Sep 1993.

[281] P. Morell and R. H. Quarles. Basic Neurochemistry: Molecular, Cellular and Medical Aspects. Lippincott-Raven, New York, 6 edition, 1999.

[282] W. T. Norton and S. E. Poduslo. Myelination in rat brain: method of myelin isolation. J Neurochem, 21(4):749-757, Oct 1973.

[283] Jorge N Larocca and Williams T Norton. Isolation of myelin. Curr Protoc Cell Biol, Chapter 3:Unit3.25, Jan 2007.

[284] W. Cammer, L. Bieler, T. Fredman, and W. T. Norton. Quantitation of myelin carbonic anhydrase-development and subfractionation of rat brain myelin and comparison with myelin from other species. Brain Res, 138(1):17-28, Dec 1977.

[285] J. C. Rios, C. V. Melendez-Vasquez, S. Einheber, M. Lustig, M. Grumet, J. Hemperly, E. Peles, and J. L. Salzer. Contactin-associated protein (caspr) and contactin form a complex that is targeted to the paranodal junctions during myelination. J Neurosci, 20(22):8354-8364, Nov 2000.

[286] J. Folch, M. Lees, and G. H. Sloane Stanley. A simple method for the isolation and purification of total lipides from animal tissues. J Biol Chem, 226(1):497-509, May 1957. 
[287] M. M. Bradford. A rapid and sensitive method for the quantitation of microgram quantities of protein utilizing the principle of protein-dye binding. Anal Biochem, 72:248-254, May 1976.

[288] P. K. Smith, R. I. Krohn, G. T. Hermanson, A. K. Mallia, F. H. Gartner, M. D. Provenzano, E. K. Fujimoto, N. M. Goeke, B. J. Olson, and D. C. Klenk. Measurement of protein using bicinchoninic acid. Anal Biochem, 150(1):76-85, Oct 1985.

[289] K. Fiedler, T. Kobayashi, T. V. Kurzchalia, and K. Simons. Glycosphingolipidenriched, detergent-insoluble complexes in protein sorting in epithelial cells. Biochemistry, 32(25):6365-6373, Jun 1993.

[290] D. A. Brown and J. K. Rose. Sorting of gpi-anchored proteins to glycolipid-enriched membrane subdomains during transport to the apical cell surface. Cell, 68(3):533544, Feb 1992.

[291] B. Bruegger, G. Erben, R. Sandhoff, F. T. Wieland, and W. D. Lehmann. Quantitative analysis of biological membrane lipids at the low picomole level by nanoelectrospray ionization tandem mass spectrometry. Proc Natl Acad Sci USA, 94(6):2339-2344, Mar 1997.

[292] B. Bruegger, B. Glass, P. Haberkant, I. Leibrecht, F. T. Wieland, and H.-G. Kräusslich. The hiv lipidome: a raft with an unusual composition. Proc Natl Acad Sci U $S$ A, 103(8):2641-2646, Feb 2006.

[293] D. Magde, E. L. Elson, and W. W. Webb. Fluorescence correlation spectroscopy. ii. an experimental realization. Biopolymers, 13(1):29-61, Jan 1974.

[294] A. J. García-Sáez, D. C. Carrer, and P. Schwille. Fluorescence correlation spectroscopy for the study of membrane dynamics and organization in giant unilamellar vesicles. Methods Mol Biol, 606:493-508, 2010.

[295] S. Chiantia, J. Ries, and P. Schwille. Fluorescence correlation spectroscopy in membrane structure elucidation. Biochim Biophys Acta, 1788(1):225-233, Jan 2009.

[296] H.-J. Kaiser, D. Lingwood, I. Levental, J. L. Sampaio, L. Kalvodova, L. Rajendran, and K. Simons. Order of lipid phases in model and plasma membranes. Proc Natl Acad Sci U S A, 106(39):16645-16650, Sep 2009.

[297] D. Fitzner, A. Schneider, A. Kippert, W. Möbius, K. I. Willig, S. W. Hell, G. Bunt, K. Gaus, and M. Simons. Myelin basic protein-dependent plasma membrane reorganization in the formation of myelin. EMBO J, 25(21):5037-5048, Nov 2006. 
[298] M. Jung, E. Krämer, M. Grzenkowski, K. Tang, W. Blakemore, A. Aguzzi, K. Khazaie, K. Chlichlia, G. von Blankenfeld, and H. Kettenmann. Lines of murine oligodendroglial precursor cells immortalized by an activated neu tyrosine kinase show distinct degrees of interaction with axons in vitro and in vivo. Eur $J$ Neurosci, 7(6):1245-1265, Jun 1995.

[299] G. Gregoriadis, editor. Liposome Technology. CRC, Boca Raton, FL, 1984.

[300] P. L. Felgner, T. R. Gadek, M. Holm, R. Roman, H. W. Chan, M. Wenz, J. P. Northrop, G. M. Ringold, and M. Danielsen. Lipofection: a highly efficient, lipidmediated dna-transfection procedure. Proc Natl Acad Sci U S A, 84(21):7413-7417, Nov 1987.

[301] E. Neumann, M. Schaefer-Ridder, Y. Wang, and P. H. Hofschneider. Gene transfer into mouse lyoma cells by electroporation in high electric fields. EMBO J, 1(7):841$845,1982$.

[302] M. Simons, E.-M. Kramer, P. Macchi, S. Rathke-Hartlieb, J. Trotter, K.-A. Nave, and J. B. Schulz. Overexpression of the myelin proteolipid protein leads to accumulation of cholesterol and proteolipid protein in endosomes/lysosomes: implications for pelizaeus-merzbacher disease. J Cell Biol, 157(2):327-336, Apr 2002.

[303] M. Minsky. Memoir on inventing the confocal scanning microscope. Scanning, 10:128-138, 1988.

[304] J. Pawley, editor. Handbook of Biological Confocal Microscopy. Springer, 1995.

[305] H. Hutter. Fluorescent reporter methods. Methods Mol Biol, 351:155-173, 2006.

[306] P. Várnai and T. Balla. Visualization of phosphoinositides that bind pleckstrin homology domains: calcium- and agonist-induced dynamic changes and relationship to myo-[3h]inositol-labeled phosphoinositide pools. J Cell Biol, 143(2):501-510, Oct 1998.

[307] Qi Li, Anthony Lau, Terence J Morris, Lin Guo, Christopher B Fordyce, and Elise F Stanley. A syntaxin 1, galpha(o), and n-type calcium channel complex at a presynaptic nerve terminal: analysis by quantitative immunocolocalization. J Neurosci, 24(16):4070-4081, Apr 2004.

[308] M. Sezgin and B. Sankur. Survey over image thresholding techniques and quantitative performance evaluation. Journal of Electronic Imaging, 13:146-165, 2004.

[309] D. J. Mueller, J. Helenius, D. Alsteens, and Y. F. Dufrêne. Force probing surfaces of living cells to molecular resolution. Nat Chem Biol, 5(6):383-390, Jun 2009. 
[310] D. J. Muller. Afm: a nanotool in membrane biology. Biochemistry, 47(31):79867998, Aug 2008.

[311] D. Axelrod, D. E. Koppel, J. Schlessinger, E. Elson, and W. W. Webb. Mobility measurement by analysis of fluorescence photobleaching recovery kinetics. Biophys J, 16(9):1055-1069, Sep 1976.

[312] D. M. Soumpasis. Theoretical analysis of fluorescence photobleaching recovery experiments. Biophys J, 41(1):95-97, Jan 1983.

[313] E. L. Snapp, N. Altan, and J. Lippincott-Schwartz. Measuring protein mobility by photobleaching gfp chimeras in living cells. Curr Protoc Cell Biol, Chapter 21:Unit 21.1, Aug 2003.

[314] A. K. Kenworthy. Fluorescence recovery after photobleaching studies of lipid rafts. Methods Mol Biol, 398:179-192, 2007.

[315] M. A. Ravier, T. Tsuboi, and G. A. Rutter. Imaging a target of ca2+ signalling: dense core granule exocytosis viewed by total internal reflection fluorescence microscopy. Methods, 46(3):233-238, Nov 2008.

[316] Systat Software Inc., San Jose, CA, USA. SigmaPlot Statistics user guide, 2009.

[317] R. C. Armstrong. Isolation and characterization of immature oligodendrocyte lineage cells. Methods, 16(3):282-292, Nov 1998.

[318] S. Nawaz, A. Kippert, A. S. Saab, H. B. Werner, T. Lang, K.-A. Nave, and M. Simons. Phosphatidylinositol 4,5-bisphosphate-dependent interaction of myelin basic protein with the plasma membrane in oligodendroglial cells and its rapid perturbation by elevated calcium. J Neurosci, 29(15):4794-4807, Apr 2009.

[319] W. Hunziker, J. A. Whitney, and I. Mellman. Brefeldin a and the endocytic pathway. possible implications for membrane traffic and sorting. FEBS Lett, 307(1):93-96, Jul 1992.

[320] A. H. Merrill, E. Wang, D. G. Gilchrist, and R. T. Riley. Fumonisins and other inhibitors of de novo sphingolipid biosynthesis. Adv Lipid Res, 26:215-234, 1993.

[321] Y. Pewzner-Jung, O. Brenner, S. Braun, E. L. Laviad, S. Ben-Dor, E. Feldmesser, S. Horn-Saban, D. Amann-Zalcenstein, C. Raanan, T. Berkutzki, R. Erez-Roman, O. Ben-David, M. Levy, D. Holzman, H. Park, A. Nyska, A. H. Merrill, and A. H. Futerman. A critical role for ceramide synthase 2 in liver homeostasis: Ii. insights into molecular changes leading to hepatopathy. J Biol Chem, 285(14):10911-10923, Apr 2010. 
[322] Y. Pewzner-Jung, H. Park, E. L. Laviad, L. C. Silva, S. Lahiri, J. Stiban, R. ErezRoman, B. Brügger, T. Sachsenheimer, F. Wieland, M. Prieto, A. H. Merrill, and A. H. Futerman. A critical role for ceramide synthase 2 in liver homeostasis: I. alterations in lipid metabolic pathways. J Biol Chem, 285(14):10902-10910, Apr 2010.

[323] I. Becker, L. Wang-Eckhardt, A. Yaghootfam, V. Gieselmann, and M. Eckhardt. Differential expression of (dihydro)ceramide synthases in mouse brain: oligodendrocytespecific expression of cers2/lass2. Histochem Cell Biol, 129(2):233-241, Feb 2008.

[324] J. Mokry, J. Karbanova, S. Filip, D. Cizkova, J. Pazour, and D. English. Phenotypic and morphological characterization of in vitro oligodendrogliogenesis. Stem Cells Dev, 17(2):333-341, Apr 2008.

[325] C. A. Dyer and J. A. Benjamins. Organization of oligodendroglial membrane sheets. i: Association of myelin basic protein and 2',3'-cyclic nucleotide 3'-phosphohydrolase with cytoskeleton. J Neurosci Res, 24(2):201-211, Oct 1989.

[326] T. A. Watkins, B. Emery, S. Mulinyawe, and B. A. Barres. Distinct stages of myelination regulated by gamma-secretase and astrocytes in a rapidly myelinating cns coculture system. Neuron, 60(4):555-569, Nov 2008.

[327] M. Haber, S. Vautrin, E. J. Fry, and K. K. Murai. Subtype-specific oligodendrocyte dynamics in organotypic culture. Glia, 57(9):1000-1013, Jul 2009.

[328] R. N. Lintner and C. A. Dyer. Redistribution of cholesterol in oligodendrocyte membrane sheets after activation of distinct signal transduction pathways. J Neurosci Res, 60(4):437-449, May 2000.

[329] J. M. Bourre, S. Pollet, O. Daudu, F. Le Saux, and N. Baumann. Myelin consists of a continuum of particles of different density with varying lipid composition: major differences are found between normal mice and quaking mutants. Biochimie, 59(10):819-824, 1977.

[330] V. Koliatsos and R. Ratan, editors. Cell Death and Diseases of the Nervous System. Humana Press, Totowa, 1999.

[331] V. L. Friedrich and E. Mugnaini. Myelin sheath thickness in the cns is regulated near the axon. Brain Res, 274(2):329-331, Sep 1983.

[332] A. E. Warrington and S. E. Pfeiffer. Proliferation and differentiation of o4+ oligodendrocytes in postnatal rat cerebellum: analysis in unfixed tissue slices using antiglycolipid antibodies. J Neurosci Res, 33(2):338-353, Oct 1992. 
[333] B. D. Trapp, A. Nishiyama, D. Cheng, and W. Macklin. Differentiation and death of premyelinating oligodendrocytes in developing rodent brain. J Cell Biol, 137(2):459468, Apr 1997.

[334] P. B. Simpson and R. C. Armstrong. Intracellular signals and cytoskeletal elements involved in oligodendrocyte progenitor migration. Glia, 26(1):22-35, Mar 1999.

[335] Jacob A Sloane and Timothy K Vartanian. Wave1 and regulation of actin nucleation in myelination. Neuroscientist, 13(5):486-491, Oct 2007.

[336] J. Y. Léna, C. Legrand, C. Faivre-Sarrailh, L. L. Sarliève, C. Ferraz, and A. Rabié. High gelsolin content of developing oligodendrocytes. Int J Dev Neurosci, 12(5):375386, Aug 1994.

[337] C. Legrand, C. Ferraz, M. C. Clavel, and A. Rabié. Immunocytochemical localisation of gelsolin in oligodendroglia of the developing rabbit central nervous system. Brain Res, 395(2):231-235, Dec 1986.

[338] J. M. Boggs, W. Gao, J. Zhao, H.-J. Park, Y. Liu, and A. Basu. Participation of galactosylceramide and sulfatide in glycosynapses between oligodendrocyte or myelin membranes. FEBS Lett, 584(9):1771-1778, May 2010.

[339] D. Brockschnieder, H. Sabanay, D. Riethmacher, and E. Peles. Ermin, a myelinating oligodendrocyte-specific protein that regulates cell morphology. J Neurosci, 26(3):757-762, Jan 2006.

[340] C. M. D. Hill and G. Harauz. Charge effects modulate actin assembly by classic myelin basic protein isoforms. Biochem Biophys Res Commun, 329(1):362-369, Apr 2005 .

[341] J. M. Boggs, G. Rangaraj, C. M. D. Hill, I. R. Bates, Y.-M. Heng, and G. Harauz. Effect of arginine loss in myelin basic protein, as occurs in its deiminated charge isoform, on mediation of actin polymerization and actin binding to a lipid membrane in vitro. Biochemistry, 44(9):3524-3534, Mar 2005.

[342] M. R. Galiano, A. Andrieux, J. C. Deloulme, C. Bosc, A. Schweitzer, D. Job, and M. E. Hallak. Myelin basic protein functions as a microtubule stabilizing protein in differentiated oligodendrocytes. J Neurosci Res, 84(3):534-541, Aug 2006.

[343] C. M. D. Hill, D. S. Libich, and G. Harauz. Assembly of tubulin by classic myelin basic protein isoforms and regulation by post-translational modification. Biochemistry, 44(50):16672-16683, Dec 2005. 
[344] P. Kursula, V. P. Lehto, and A. M. Heape. The small myelin-associated glycoprotein binds to tubulin and microtubules. Brain Res Mol Brain Res, 87(1):22-30, Feb 2001.

[345] E. Paluch, J. van der Gucht, and C. Sykes. Cracking up: symmetry breaking in cellular systems. J Cell Biol, 175(5):687-692, Dec 2006.

[346] J. Bereiter-Hahn, M. Lück, T. Miebach, H. K. Stelzer, and M. Vöth. Spreading of trypsinized cells: cytoskeletal dynamics and energy requirements. J Cell Sci, 96 ( Pt 1):171-188, May 1990.

[347] K. Burton and D. L. Taylor. Traction forces of cytokinesis measured with optically modified elastic substrata. Nature, 385(6615):450-454, Jan 1997.

[348] G. T. Charras, J. C. Yarrow, M. A. Horton, L. Mahadevan, and T. J. Mitchison. Non-equilibration of hydrostatic pressure in blebbing cells. Nature, 435(7040):365369, May 2005.

[349] T. C. Sudhof. The synaptic vesicle cycle. Annu Rev Neurosci, 27:509-547, 2004.

[350] A. Bolis, S. Coviello, I. Visigalli, C. Taveggia, A. Bachi, A. H. Chishti, T. Hanada, A. Quattrini, S. C. Previtali, A. Biffi, and A. Bolino. Dlg1, sec8, and mtmr2 regulate membrane homeostasis in schwann cell myelination. J Neurosci, 29(27):8858-8870, Jul 2009.

[351] T. Lang. Imaging ca2+-triggered exocytosis of single secretory granules on plasma membrane lawns from neuroendocrine cells. Methods Mol Biol, 440:51-59, 2008.

[352] T. L. Fletcher and G. A. Banker. The establishment of polarity by hippocampal neurons: the relationship between the stage of a cell's development in situ and its subsequent development in culture. Dev Biol, 136(2):446-454, Dec 1989.

[353] C. A. Dyer, T. M. Philibotte, M. K. Wolf, and S. Billings-Gagliardi. Myelin basic protein mediates extracellular signals that regulate microtubule stability in oligodendrocyte membrane sheets. J Neurosci Res, 39(1):97-107, Sep 1994.

[354] J. M. Boggs, W. Gao, and Y. Hirahara. Signal transduction pathways involved in interaction of galactosylceramide/sulfatide-containing liposomes with cultured oligodendrocytes and requirement for myelin basic protein and glycosphingolipids. $J$ Neurosci Res, 86(7):1448-1458, May 2008.

[355] F. Monnet-Tschudi, M. G. Zurich, O. Sorg, J. M. Matthieu, P. Honegger, and B. Schilter. The naturally occurring food mycotoxin fumonisin b1 impairs myelin formation in aggregating brain cell culture. Neurotoxicology, 20(1):41-48, Feb 1999. 
[356] T. D. Bird, D. F. Farrell, and S. M. Sumi. Brain lipid composition of the shiverer mouse: (genetic defect in myelin development). J Neurochem, 31(1):387-391, Jul 1978.

[357] C. A. Dyer, T. M. Philibotte, S. Billings-Gagliardi, and M. K. Wolf. Cytoskeleton in myelin-basic-protein-deficient shiverer oligodendrocytes. Dev Neurosci, 17(1):53-62, 1995.

[358] M. Wiktorowicz and A. Roach. Regulation of myelin basic protein gene transcription in normal and shiverer mutant mice. Dev Neurosci, 13(3):143-150, 1991.

[359] P. F. F. Almeida, A. Pokorny, and A. Hinderliter. Thermodynamics of membrane domains. Biochim Biophys Acta, 1720(1-2):1-13, Dec 2005.

[360] H. Lodish, A. Berk, P. Matsudaira, C. A. Kaiser, M. Krieger, M. P. Scott, L. Zipursky, and J. Darnell. Molecular cell biology. New York : W.H. Freeman and Company, 2003. 


\title{
Curriculum Vitae
}

\author{
Larisa Yurlova
}

born $1^{\text {st }}$ October 1981 in St. Pertersburg, Russian Federation

Obere Masch Str. 14, 37073 Göttingen

E-Mail yurlova@em.mpg.de

\section{Education and Research Experience}

2007 - present Max Planck Institute of Experimental Medicine, Göttingen, Germany: AG Simons. PhD thesis: Myelin Membrane Growth and Organization in a Cellular Model System

2004 - present Georg-August-University, Göttingen, Germany: International $\mathrm{MSc} / \mathrm{PhD}$ Molecular Biology program. Master's examination grade 1.15 (A, excellent), direct admission to $\mathrm{PhD}$

2002 - 2004 Sechenov Institute, Russian Academy of Sciences, St. Petersburg, Russian Fed.: junior staff scientist

1998 - 2004 St. Petersburg State University, St. Petersburg, Russian Fed.: Biological Faculty, Dept. Biochemistry. Bachelor's degree in Biology, grade 4.5; grading scale 1 to 5 (best)

1988 - 1998 School-Gymnasia N52, St. Petersburg, Russian Fed. Secondary complete general education (Abitur), grade 5 (excellent), honors

Scholarships \& Grants

$2005-2008$

EU Marie Curie Fellowship NEUREST

$2004-2005$

Stipend International Max Planck Research School

2002 and 2003 Orbeli Scholarships (research scholarship of Russian Acad. of Sciences) 2003 Research grant for graduate/postgraduate students of St. Petersburg 